1 POPULAR HANDBOOK

$$
\text { jo ThE Microscope }
$$

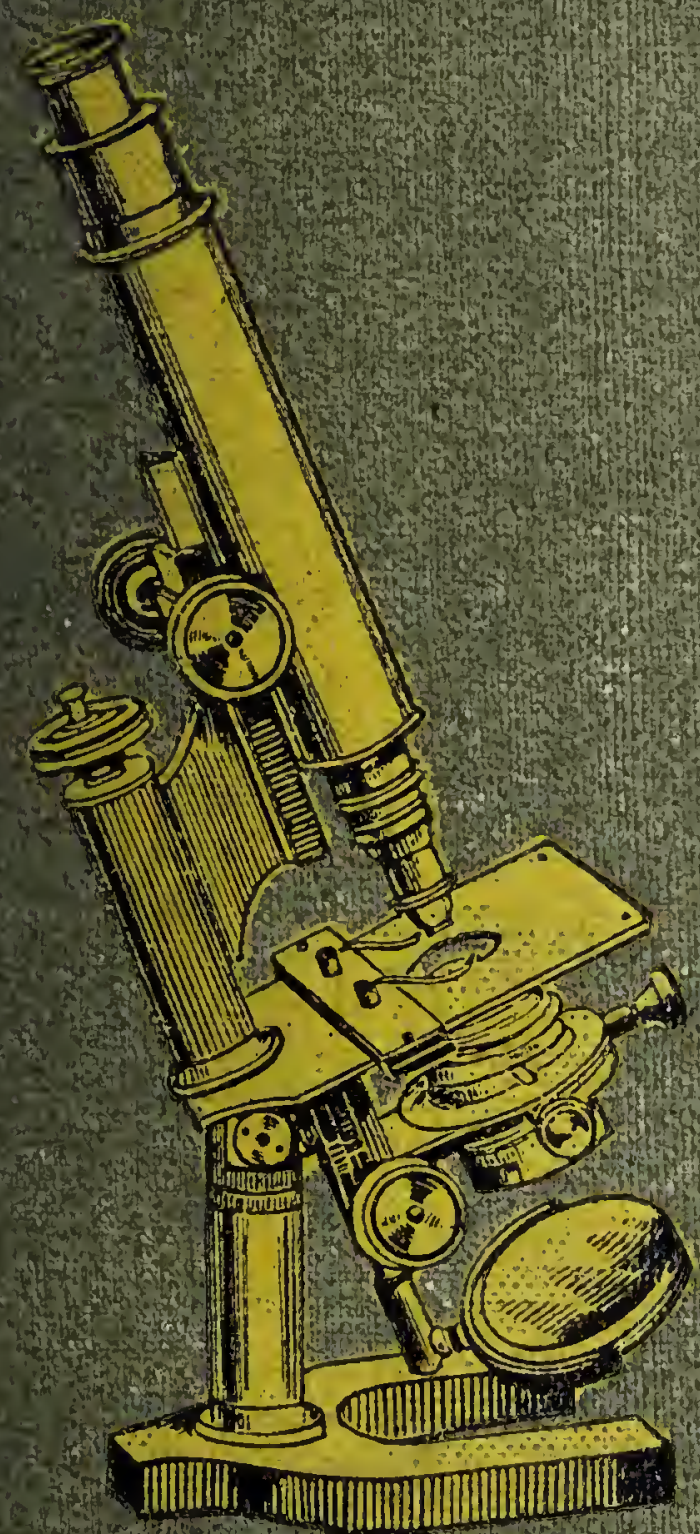

\title{
LEWIS WRIGH:
}




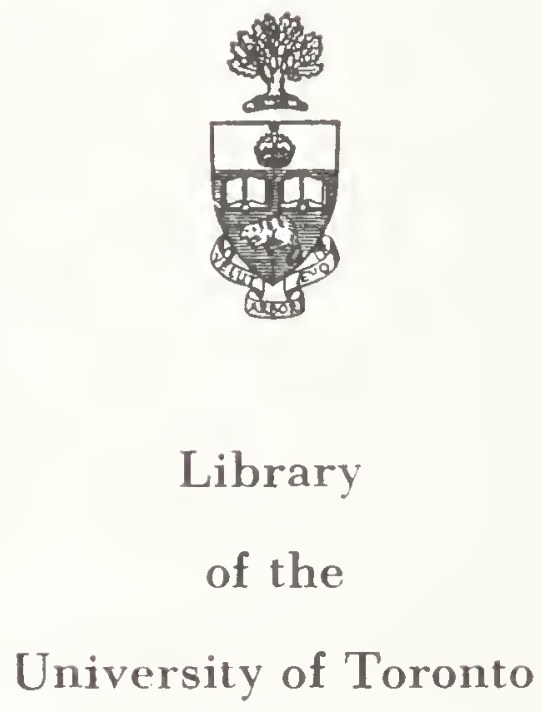



Digitized by the Internet Archive in 2018 with funding from University of Toronto 


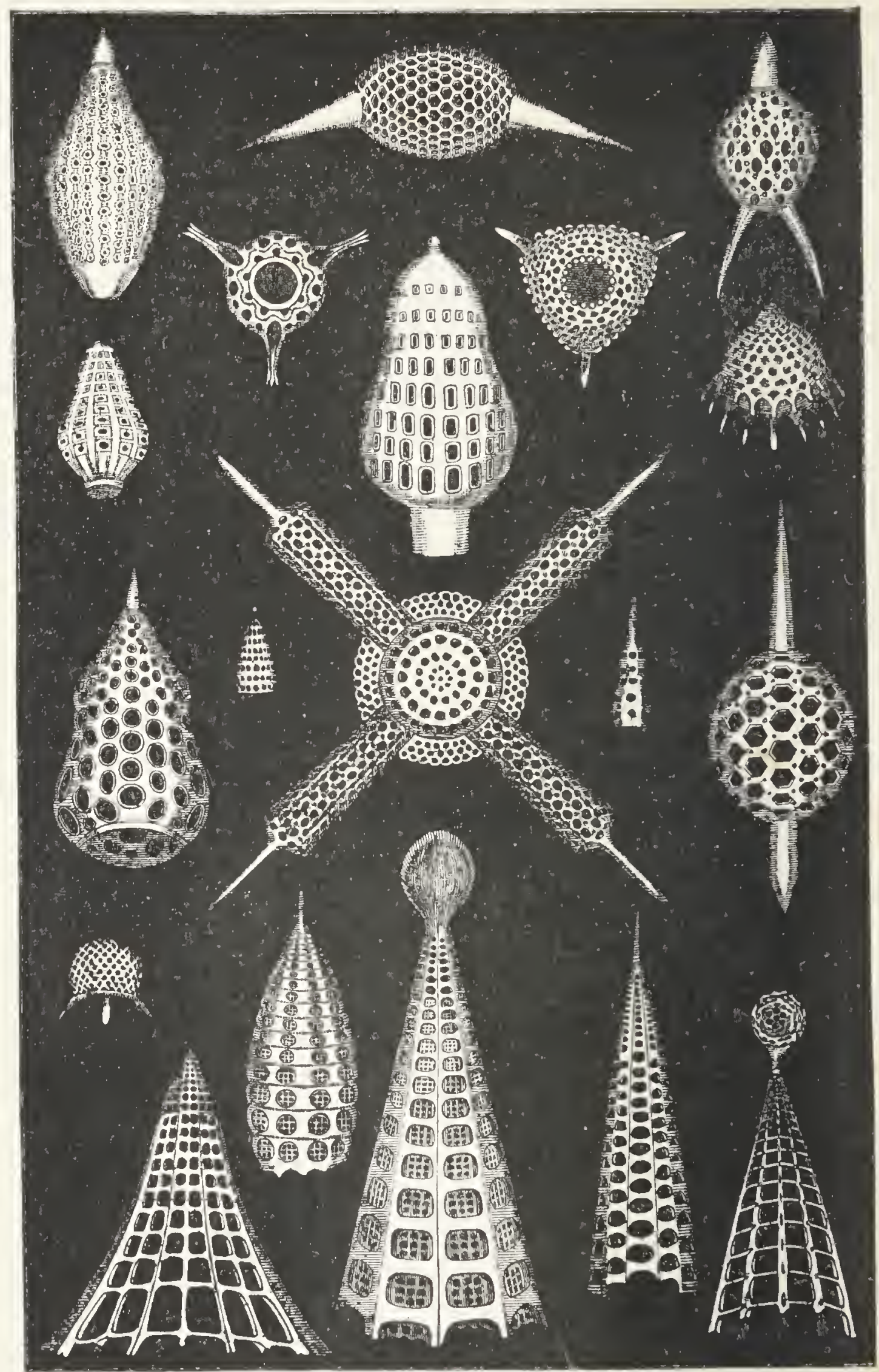

Mungo Pontor, del. 


\section{A POPULAR HANDBOOK}

\section{THE MICROSCOPE}

BI

\section{LEWIS WRIGHT}

AUTIOR OF "LIGHT: A COURSE OF EXPEIIMEXTAL OPTICS"

'optical projection' etc.

\section{LONDON}

THE RELIGIOUS TRACT SOCLETY 56 Paternoster Row, and 65 St Paul's Churchyard 
' The wistom of God receives small honour from those that stare about and with gross rusticity admire His works: those highly magnify Him, whose judicious inquiry into His acts, and deliberate research into His creations, return the duty of a devout and learned [i.e. intelligent] admiration.'

Religio Medici, A.D. 1686. 


\section{INTRODUCTION}

No excuse is needed for any attempt to promote the better study of Nature; and if it be thought that the Society for which this book is written requires such, it may be found in the passage I have chosen to preface it.

But the Microscope has special claims upon such a Society. Chalmers long ago pointed out ${ }^{1}$ that whereas the stupendous revelations of the Telescope had been used to support a class of doubts and negations, which we have seen repeated only yesterday in sneers at "the anthropocentric theory' of the universe, the Microscope had even then furnished reply to such arguments, by enabling us to 'see a world in every atom.' So also in our own day, when Haeckel and Spencer had taught that Life began in chemical combination into more and more ' complex molecules,' whose mere complexity ${ }^{2}$ added Life to previous properties of Matter, in either 'primeval slime' ${ }^{3}$ or lowly living beings 'without organisation or structure,' it was the Microscope

1 Astronomical Discourses, No. III.

${ }^{2}$ See it all summarised in The Story of Creation, by MIx. Edward Clodd. I have discussed this ignorant dogmatism at length in Contemporary Review, August, 1888.

${ }^{3}$ Huxley's Bathybius. This was soon exploded: a simple chemical analysis of the stuff did that. But it was distinctly the biassed (and therefore unscientific) predisposition to this view of Life which led so able a man to believe in it without the slightest experimental investigation, and leaving to others such simple analysis as finally disposed of it. 
that proved such teaching due to sheer ignorance and not to superior knowledge, and, if it could not reveal the Divine mystery of living existence, at least manifested it to us as a greater Mystery than ever.

The Microscope, then, has deserved well of the Christian believer; and it is to be hoped that this may not be the last work the Religious Tract Society may see fit to publish concerning the marvels it unfolds to us, upon which part of the subject very limited space has prevented any enlargement in this little volume. For space has made my task a difficult one, every page requiring anxious consideration as to how best to use it.

In those devoted to apparatus, it seemed most useful to follow the example of my friend the late Dr. Carpenter, and show each reader straightforwardly, what he could get for a given expenditure. In presenting such types of instrument as alone fall within the plan of a book only elementary and introductory, there has been absolute impartiality. There is indeed hardly a manufacturing firm of standing, from which I have not received some personal kindness, and there are several to whose workshops I have been frankly admitted, long before this book was proposed to me. As I knew enough of tools and workmanship in my youth to have made a fair microscope myself had I cared, such means of forming a practical judgment have been valuable; and since every stand mentioned has been carefully examined, confidence may be placed in the opinions expressed, even where (as in some cases) they differ from others as to the merits of certain details. Of objectives also I have had unusual opportunities for judging, a very large number having passed through my hands, including I believe nearly all of any repute, and in many cases the very first specimens of lenses now well known 
and valued. Every lens named is familiar to me, and the list (again necessarily almost confined to the more moderate prices) will be found both catholic and trustworthy.

As to manipulation, endeavour was made chiefly to recall from my own early experience what the beginner most needs to know, and to tell him about it so simply that he may get to know it, for practical purposes. The experienced worker is not pretended to be instructed in this book; and the beginner who uses it will be the best judge how far its more modest objects have been attained.

The later chapters are necessarily only outlines: opinions may well differ as to how these have been drawn, though no part received more anxious consideration. A miscellaneous illustrated list of 'objects' did not promise to be of any particular use : any really systematic treatment was forbidden by the narrow limits: what was it best to do? The idea finally adopted was to endeavour to make students, rather than to teach them. For this, it seemed best to blend into such broad and popular descriptions as might perhaps interest even a general reader, hints and suggestions as to personal courses of study, or lines of investigation, or even systematic and intelligent in place of random collection of objects - to suggest, in fact, definite work to do, which might maintain the Microscope in its place as a valued companion through life. Some attempt is at the same time made to convey a sense of that unity of Nature, through all its diversity, in which the present writer profoundly believes.

My sincere thanks are due to Messrs. Beck, Swift, Baker, and Watson, for having, besides their own stands (which makers are naturally willing to advertise) placed at my disposal numerous illustrations of those subsidiary appliances common to all. They are more specially due to 
Messrs. Beck for allowing me to photograph the late Mr. R. Beck's unrivalled original drawings of Arachnoidiscus and of the 'test' Podura scale; and to Mr. C. L. Curties for negatives of $N$. lyra and Heliopelta. To Messrs. Partridge \& Co. and Dr. Dallinger I owe the two fine drawings by the latter referred to in their place; and in the descriptions of Water Fleas and Insects, I have drawn freely upon a series of excellent microscopical drawings by Mr. Arthur Hammond, F.L.S. The Frontispiece is a selection taken entirely from an exquisite set of illustrations ${ }^{1}$ drawn under the microscope with his own hand by the late $\mathrm{Mr}$. Mungo Ponton, who will be remembered amongst photographers for his discovery of the photographic sensitiveness of potassic bichromate, and from whom I received when a lad the kindest personal assistance regarding my earlier experiments in optics and physical science. Some of the specimens shown are almost unique; and the beauty of the drawings will speak for itself.

September, 1895.

1 For The Beginning, its When and its How (now out of print). 


\section{CONTENTS}

CHAPTER

TACE

I. Rays, Images, and Lenses . . • . . 11

II. Practical Optics of the Microscopf • • • 19

III. The Simple Microscope and its Use . • • 37

IV. The Compound Microscope and Accessories • . 44

V. Microscope Manipulation . . . . . 81

VI. Microscopical Drawing, Measurement, and PhoTOGRAPHY . . . . . . . . . 109

ViI. Manipulation, Preparation, Mounting, and SelecTION OF OBJECTS . . . . . . . 117

VIII. Pond and Marine Life . . . . . . 137

IX. The Insect World . . . . . . . 172

X. The Vegetable World . . . . . . 200

XI. Vertebrate Physiology . . . . . . 218

XII. Miscellaneous Microscopic Objects . • . . 233

XIII. Microscopical Exhibitions . . • . . 247

INDEX • . . . . . . . . 253 



\section{A POPULAR HANDBOOK}

\section{To \\ THE MICROSCOPE}

CHAPTER I

RAYS, IMAGES, AND LENSES

Iт will help the reader, both in the selection and use of his microscope and accessories, to comprehend the main principles upon which optical images depend.

1. Images and Magnification.-For anything to be 'seen,' an image or picture of it has to be thrown upon the sensitive surface at the back of the eye-ball, known as the retina, as really as a magic-lantern picture upon the white sheet. The apparent size of a thing seen depends upon that of this image ; and to 'magnify' an object is to make the picture of it on the retina larger than it would be otherwise.

As regards the eye itself, which in all essentials is like a

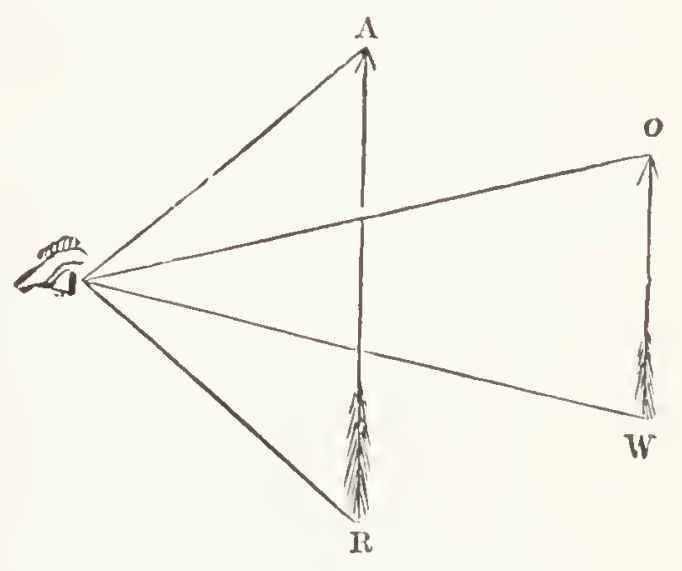

FIG. 1.- Visual Angle camera, with its lens in front and screen at the back, the apparent size of a thing depends upon the visual angle, or angle subtended by straight lines from its boundaries A $\mathrm{R}$ or ow (fig. 1), and the pupil of the eye. So the simplest way of magnifying anything is to bring it nearer the eye: the nearer 
it is, the larger it looks. We are not always fully conscious of this, because we allow for its distance, and thus thinte its size as well as see it; but direct comparison will show it. Hold a piece of money at arm's length, and see how little of the landscape it covers. Now bring it nearer-we thint it looks the same size; but, looking beyond, we see how much more of the view it covers. Bring it close to the eye, and it blots out almost all we can see of earth and heaven. There are people who look at money this way every day of their lives.

We can literally 'magnify' tiny objects in this way. The distance of sharpest vision for small objects with most people (till from age they get more long-sighted) is about 10 inches; and this is taken as the standard for judging all magnitudes in microscopy. Much closer, very few can see sharply or 'in focus.' But by looking through a small pinhole in a card we can see a thing almost close. Put a few cheese-mites within a small ring of card (to keep them from being crushed) between two bits of glass pinched together with finger and thumb, and look at them as closely as possible through the pin-hole. There will be no mistake about the magnification so obtained, which is measured by the ratio of the distance to 10 inches; at 1 inch distance it is ten times, which is expressed as ten diameters, or $\times 10{ }^{1}$

2. Images formed by Rays.--Let us now think a minute about the 'image' on the retina. We say it is formed by the lens-front of the eye, just as the image in a telescope or microscope is by its lenses. We must clearly grasp the simple but wonderful fact, that only because every ray of light has the power of forming an image, are lenses able to do so.

It is easy to prove this. Light a candle in a dark room, and support a sheet of white card or paper 2 feet away. Take also a large blackened card, and prick a pin-hole in the

I Foolish statements often made about magnifications of 'millions' reckon the surface of the object, or the squares of the diameters. The so-called 'million times' is really 1,000 diameters, or $\times 1,000$. Microscopists never use any other measure than the latter. 
middle, and hold this about midway between candle and white card. We have only just separated off a few rays by themselves, to see what they do; but we see unmistakably an image or real picture of the flame on the white card.

3. Inversion and Size of Image.-This image is upside down, and the reason is evident from fig. 2. Each point in the object o sends its rays straight through the pin-hole A to the image I; so the ray from the top goes to the bottom, and vice versa. It will also be observed that the size of the image must be in proportion to its distance from the pinhole compared with that of the object. Move the white card more away, or the

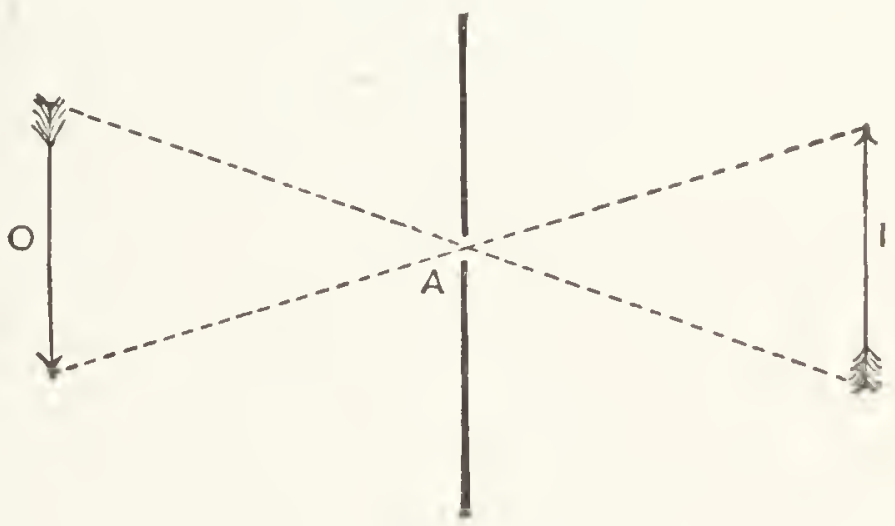

FIx. 2.-Invertel Image

black one nearer the candle, and the image is larger, and of course more faint also.

This simple fact about rays is true about all images, in the most complicated apparatus. No matter for the moment what 'rays' are; just now we need only remember that (1) they travel in straight lines so long as they traverse undisturbed the same medium (as air or glass); and (2) that objects are visible by rays from each point, which diverge or proceed from that point in all directions (where not stopped by something, or by the object itself) into any transparent medium.

4. Refraction of Rays.- The rays can, however, be readily reflected into some other direction, as by a mirror (image reflection), or by other objects (which we then see by this 'scattered reflection,' when not self-luminous). And what is more important to us, they can be bent aside, or refracted, by the surface of any fresh transparent medium of different 
density, through which they then travel in some other straight line. In such cases we must remember that we appear to ' see' the object in the final direction from which the rays reach the eye. Thus we 'see' anything in the looking-glass, and not in its real direction; and thus a stick half in the water appears bent, and the bottom much higher up than it really is.

5. The Law of Refraction.-The law of this refraction is very important. Let us take water and air, and let $\mathrm{w}_{1}$ represent the surface of the water, and draw a perpendicular,

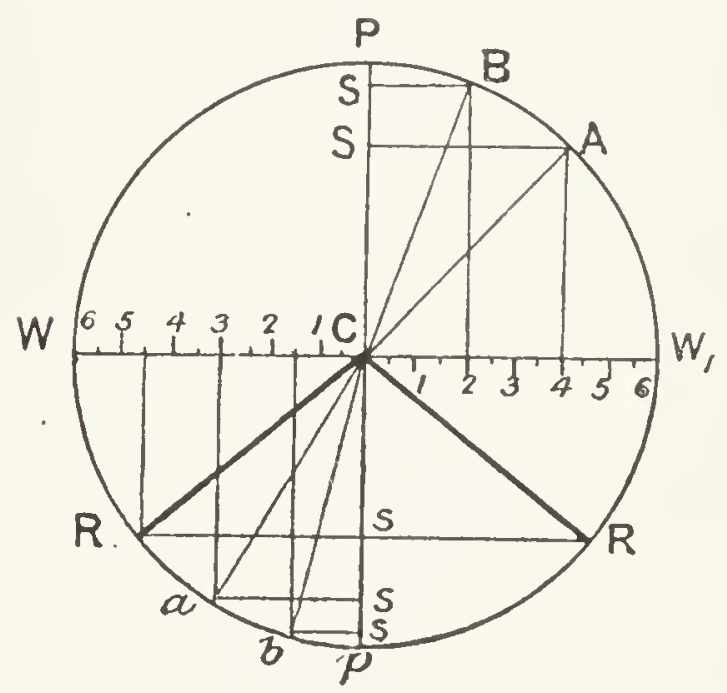

Fig. 3.-Law of refraction $\mathrm{P} p$, through some point, $\mathrm{C}$, where every ray is to strike the water, and also describe a circle round c. Any ray, P C, striking the surface perpendicularly, goes straight on to $p$. Take some other ray, A C, striking the water at an angle $\mathrm{A} \mathrm{CP}$ with the perpendicular. ${ }^{1}$ Part of it will be reflected at an equal angle, as if the surface were a mirror; but the part that enters the water is refracted at $\mathrm{c}$ to $a$, down towards the perpendicular, because the water is denser than the air. ${ }^{2}$ That is the first law. In the denser medium the rays are refracted towards the perpendicular. Conversely, on entering a rarer medium, they are refracted from the perpendicular, so that a ray from $a$ to $\mathrm{C}$ in the water would be refracted to $\mathrm{A}$. This involves another important fact, that if we trace a ray the other way, its path is exactly the same, or it is reversible.

1 Optical angles are measured from the perpendicular, called the 'normal,' and not with the surface.

2 The optical density is meant. As a rule heavier fluids or glusses refract more, but not always. Thus oil of turpentine floats on water, but lias more optical refractive power. 
But the measure of the refraction is most important to us. Draw the line As from where the ray cuts the circle, at right angles to the perpendicular $\mathrm{P} \mathrm{C}$; then $\mathrm{A} S$ is the geometrical sine of the angle $\mathrm{ACP}$. Divide $\mathrm{CW}$ and $\mathrm{CW}_{1}$ each into six parts numbered, and let the ray be such that a perpendicular from $\mathrm{A}$ meets $\mathrm{C}_{1}$ at the fourth division: this gives the measure of $\mathrm{AS}$ on $\mathrm{CW}_{1}$ as on a scale. Treat the sine $a s$ of the refracted ray $\mathrm{C} a$ in the same way; we shall find $a s$ (in water) measures three divisions. As and $a s$ are in the proportion of $4: 3$. Now take some other inclined ray $\mathrm{B} \mathrm{C}-$ for convenience we take it here so that the sine $\mathrm{B} \mathrm{S}$ of its angle is exactly half a s, or two divisions. Then the refracted ray $\mathrm{c} b$ will be such that its sine $b s$ will also measure half of $a s$. And so with any other rays; the sines of the incident and of the refracted rays are always in the same proportion of $4: 3$.

It is the same with all transparent media, including glass. The proportion differs in each, according to its density, so that in crown glass the ratio is about $3: 2$. But the ratio is the same for all rays, and that enables us to put the ratio into a simple number compared with air as 1 , and call that number the 'refractive index' of the fluid or glass. ' Thus the index of ordinary optical crown glass is 1.516. And when we know the index, it is easy to calculate the direction in which a ray entering at any angle will go.

\section{Total Reflection: Critical Angle.-The diagram} shows how fast refraction increases with the angle, and this fact brings us to a curious limit. Let the air-ray just graze the surface $\mathrm{w}_{1} \mathrm{C}$, then this surface itself is the sine, or 6 divisions. The refracted ray (in water) must be $\mathrm{CR}$, sine $4 \frac{1}{2}$ divisions. Conversely, the ray $\mathrm{R} \mathrm{C}$ will just escape from the water in the path $\mathrm{CW}_{1}$. If, then, we take some ray in the water of greater angle than $\mathrm{RC} p$, what is to happen to that, since it cannot get out of the water? It does not do so, but is totally reflected back at the same angle, with a 
brilliance no mirror can equal; as can be seen by holding high a tumbler of water, and observing the bright reflection from under the surface. This reflection at the surface of a rarer medium is the only case of total reflection of rays in nature; but the very important point to us is that rays cannot get out of a denser medium, beyond what is called the 'critical angle,' R C R. This angle is plainly less, the denser the medium. In water the critical angle is $97 \frac{1}{2}^{\circ}$, in crown glass $821^{\circ}$.

7. Action of a Lens. - We see things undisplaced through a window, because the rays which are bent towards the perpendicular on entering the pane of glass, are bent just as much back again on coming out, and so resume their

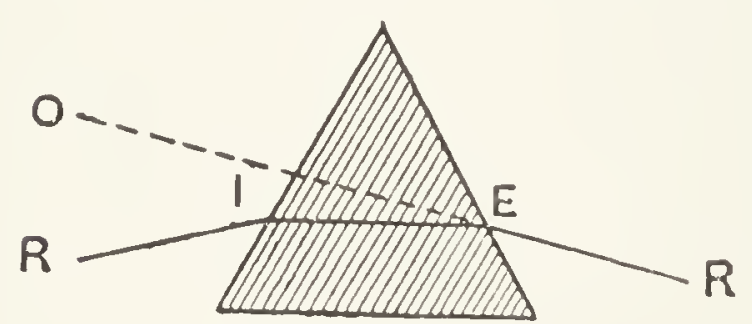

Frg. 4.-Prism original direction. But if the glass has inclined surfaces (called a prism), the rays take quite another direction on emerging, and are permanently refracted. Fig. 4 is the section of a prism. The ray $\mathrm{R} I$ is refracted towards the normal in the glass to $\mathrm{I} \mathrm{E}$, and on emergence it is refracted again away from the normal in the line $\mathrm{E} R$, and an object at $\mathrm{R}$ would be 'seen' in the direction $\mathrm{R} O$. The ray is refracted towards the thick side of the wedge or prism. A three-cornered glass 'lustre' will show this excellently; and, if a candle flame be the object, will also show vividly that the refracted beam of light is widened out into a band of colours called the spectrum. This however we leave to the next chapter.

Now a lens with its curved surfaces is practically a circular prism, whose faces become more and more inclined towards the edges. There are six possible forms of a single lens, shown in fig. 5, and it will be observed that the first three are thickest in the middle, which are called generally 
'convex' lenses; while the last three are thinnest in the middle, and are 'concave' or 'negative' lenses. Suppose we take a double convex lens-the usual form of a common magnifying or reading glass - and hold it in the parallel rays of the sun as in fig. 6. The centre ray strikes perpendicularly and is not re-

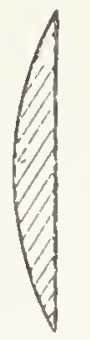

B

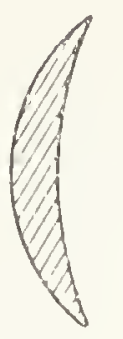

C

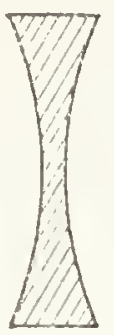

D

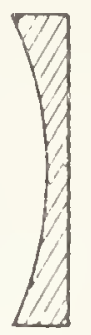

E $F$

Fig. 5.-Forms of Lenses fracted. Rays a little way from the centre strike the surface at an angle, and therefore are refracted towards the thicker part or centre, as in fig. 6. Rays farther out strike at a greater angle and are more refracted, and so on ; and the result is that all meet pretty

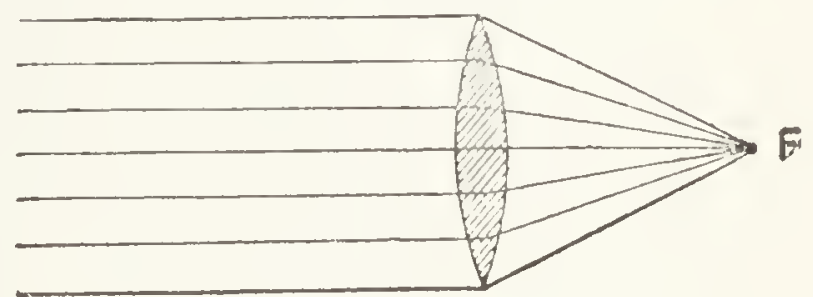

FIG. 6 nearly in the same point $\mathrm{F}$, which is the "focus for parallel rays' (known also as the focus), and where the collected sun-rays will burn a hole in a black coat.

The rays being reversible ( $\$ 5)$ if the rays proceeded from a very bright point (such as an arc lamp) at $\mathrm{F}$, they would emerge on the other side of the lens as a beam of parallel rays like the sunbeam. If the arc lamp were placed nearer

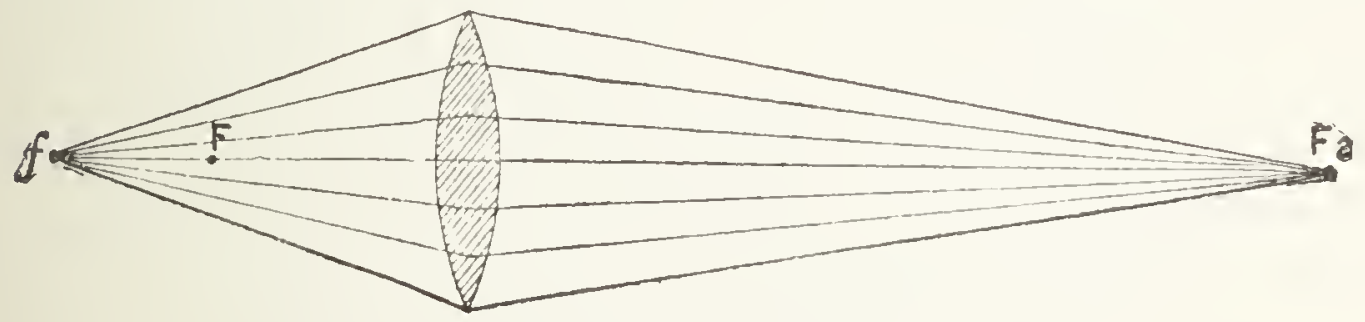

FJG. 7.-Conjugate Foci

the lens than $F$, the rays would still be refracted inwards, but evidently not enough so to be parallel : they would still be divergent, but less so. On the other hand, let the arc 
lamp be drawn back farther than $\mathrm{F}$, to some other point $f$ (fig. 7). Again, they will not issue parallel, but this time must meet again in some other point $\mathrm{F}_{2}$, which must have some fixed distance relative to $f$, according to the curves and density of the lens. So $\mathrm{F}_{2}$ is called the conjugate focus of $f$, because such foci always are in pairs; and according to the law of reversibility, if the are was at $\mathrm{F}_{2}$ the other focus must be at $f$.

Of course concave lenses, being thickest at the edges, cause rays to diverge, and therefore have no actual focus.

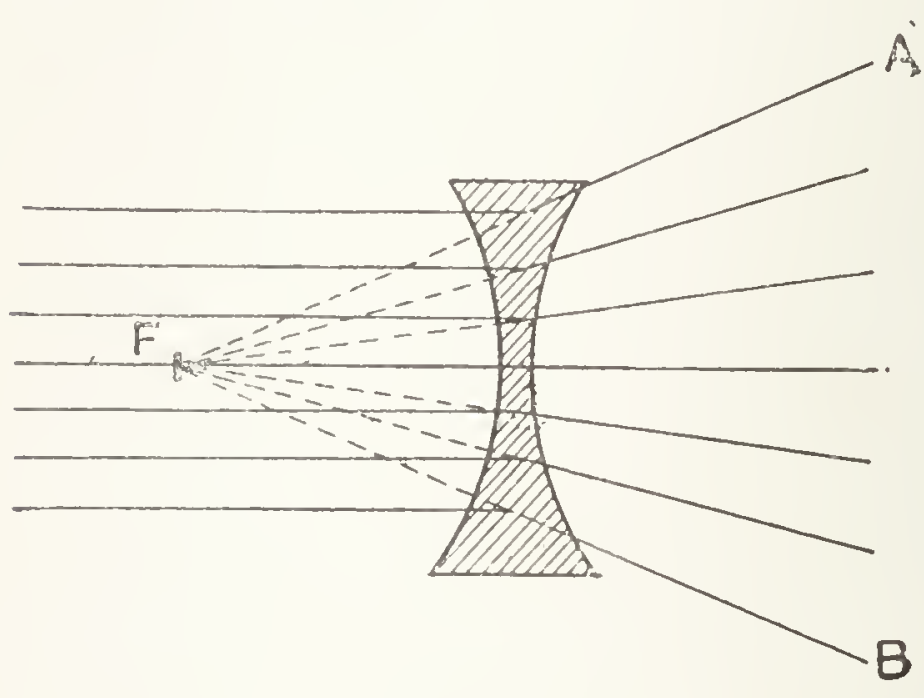

FIG. 8

What is called their 'negative focus' is, however, measured from parallel rays in the same way. If the parallel rays falling on the lens are refracted as at $\mathrm{AB}$, these rays are produced backwards on the other side of the lens to $\mathrm{F}$, and the distance from $\mathrm{F}$ to the lens is called its negative focus (fig. 8).

8. Lenses and Images.-If a reading glass of about 3 inches diameter be at hand, a simple experiment will now show us exactly what a lens really has to do with images. Light the candle again in the dark room, and set up the white card picture screen, but this time prick four more pinholes in the black card round the first one, an inch or less from it. ${ }^{1}$ If the reading glass be about the usual 10 inches focus, put the black card about 8 inches from the candle, with the centre pin-hole about level with the flame, and the white card about 3 feet beyond. There will now be seen five

1 The lens must consideriably more than cover over all the holes made 
inverted images of the flame on the white card, showing that each ray (or practically each tiny pencil of rays) forms its own separate image. First, hold the reading glass close to the holes, and it will be seen how the refraction of the lens bends in the outer four images, very much nearer the middle one. Gradually move the lens towards the white card, and they approach more and more; till a point is found where all the five images mite in one, and this is five times as bright as each was by itself. The lens is now 'focussed' on the white card; and we may now prick more holes, and finally remove the black card, and the one image is still there, but much brighter still, because the lens converges every ray that falls on its surface to the same spot on the white card. So far as the lens gathers them up, all the ray images combine into one.

This is the true action of a convex or image-forming lens. It determines a place where a cone of rays diverging from any point in an object, shall recombine at another point, in one image; and as its position marks the place where the rays are refracted so as to do this, it marks also the place where the rays from opposite points of an object cross and invert the image. Thus the relative sizes of image and object measure from the optical centre of the lens, according to the ratio of the two conjugate foci. But the lens does no more than thus converge and combine into one, veritable images potentially formed by the mere bare rays.

\section{CHAPTER II}

PRACTICAL OPTICS OF THE MICROSCOPE

WE come now to certain optical principles belonging to the microscope specially, which should be understood if the best choice is to be made amongst the instruments and accessories offered to our notice. 
9. The Simple Microscope.-The action of this instrument, or a simple 'magnifying glass' in any form, will be easily understood after the last chapter. Let the arrow ad represent an object $\mathrm{O}$, and $\mathrm{L}$ a lens held in front of the eye $\mathrm{E}$ (fig. 9). From one point $a$ in the object, diverging rays $a b$ and $a c$ proceed, and are so converged by the lens as to enter the pupil of the eye almost parallel, which is required for distinct vision. The rays thus parallelised are brought to a focus at $z$ on the retina $\mathrm{R}$, forming there the visual

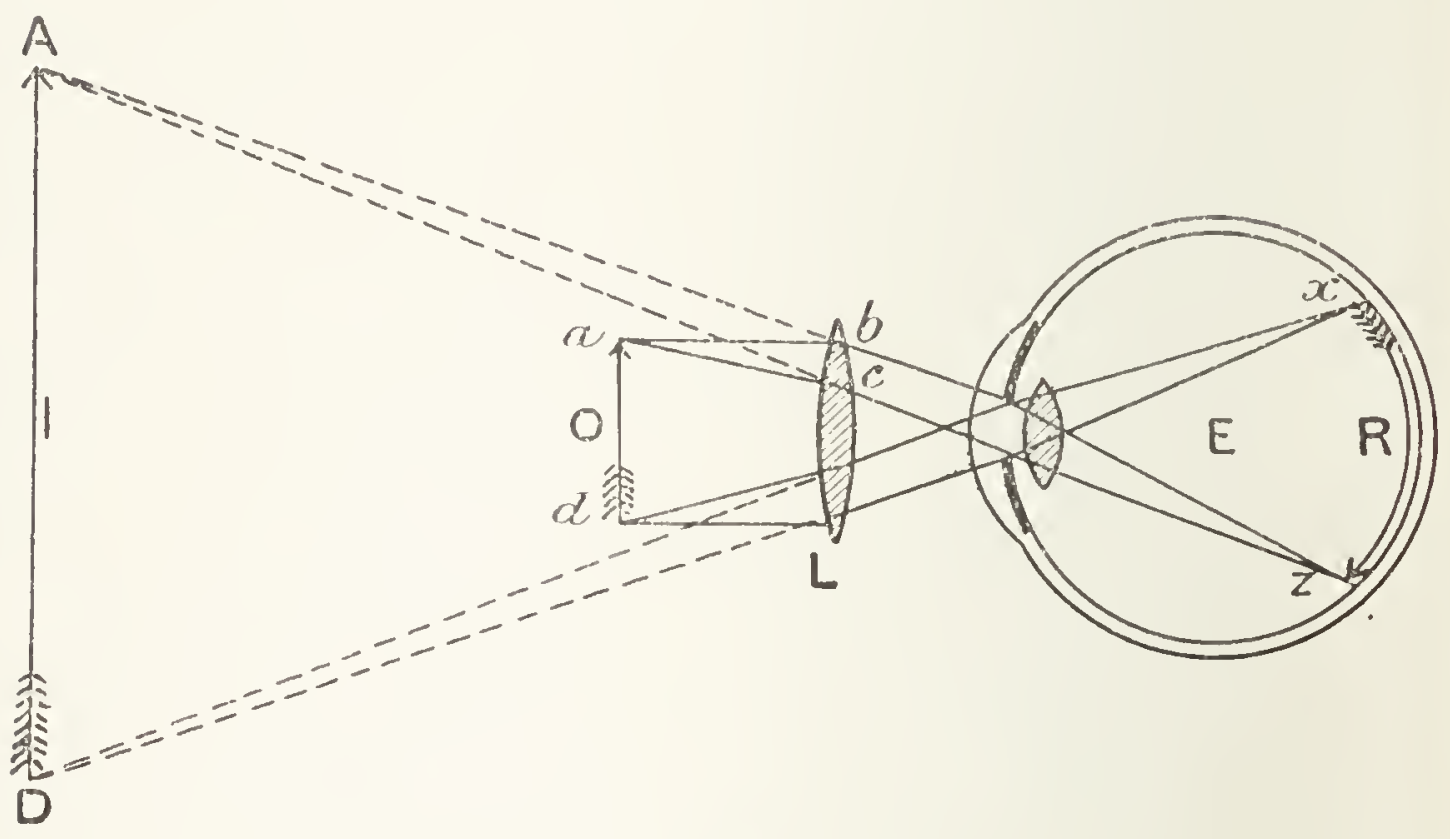

FIG. 9.-Action of Magnifying Glass

image of $a$. The image of $d$ is imaged at $x$ in the same way. But the object being 'seen' in the direction of the rays which last enter the eye $(\S 4)$ if we produce these till they meet, they appear to proceed from the points A D, which is the apparent image I of the object $a d$, as apparently seen at the distance of 10 inches. It is never to be forgotten, that the last stage in every optical combination is the formation of this image on the retina at $\mathrm{R}$, and that the final rays which must enter the pupil in order to this, have to be rendered so slightly divergent as to be practically parallel. 
10. The Compound Microscope.-In this instrument, known in general as the microscope, and the general plan of which is shown in fig. 10, a real enlarged image of the object $o$ is formed by one lens (called the object glass or objective) in the air. This enlarged image is looked at through another lens called the ocular or eye-piece, and by it further enlarged in the manner of the simple microscope. For reasons to be seen immediately, each of these lenses is always compound, and may be of various forms; but it is well to notice at once the form of eye-piece shown in the diagram. and called the Huygenian or 'negative' eye-piece. It is called negative because the image looked at is formed within it, or between its two lenses; and some form of it is more used than any other.

It consists of a larger plano-convex lens $f f$ called the field-lens, a smaller one $e e$ called the eye-lens, and a diaphragm or stop at $b b$, in the focus of the eyelens. Three pencils of rays are drawn from the centre and each end of the object $o$, which if let alone would form an inverted image at $a c$. This aerial image 'in space' behaves in one respect differently from a real object, which sends out wide cones of rays in all directions ( $\$ 3$ ). The image, on the contrary, is formed

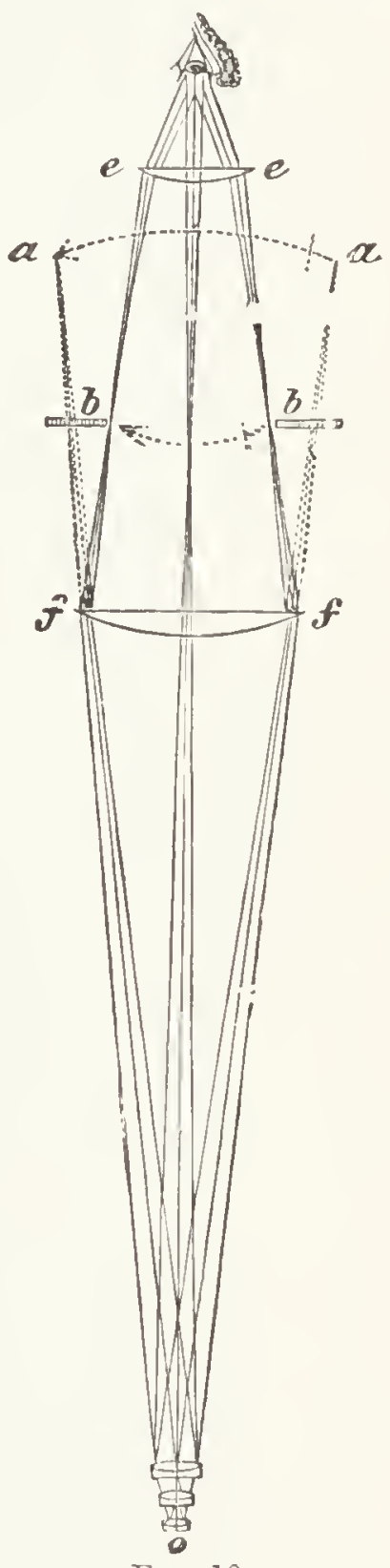

Fig. 10

by the narrow convergent pencils $o a$, which continue on after meeting or focussing. So if we looked at the image by the lens $e e$ alone, only rays from the centre of the image could pass through this lens and reach the eye. But the field-lens bends in the pencils $f a$ to $f b$, also shortening the 
focus a little, forming a rather smaller image at $b 6$, and so altering matters that the rays $f a$ are now all converged to pass through the eye-lens $e$ e. Besides this, the 'aberrations' presently mentioned are nearly corrected by this simple form of eye-piece. And lastly, as the rays begin to be converged later by the edges of the lens $f f$ than by the centre, the conjugate focus is rather extended there, and so this eyepiece helps to 'flatten the field' (\$ 36).

It will be obvious that the field-lens $f f$ belongs in principle to the objective, taking a share in the focal convergence of the image-forming rays. But it is always reckoned as part of the eye-piece.

11. Power of a Microscope.-This is ralculated by multiplying the original magnification of the image $a$, by the further magnifying power of the eye-piece. If an objective of $\frac{1}{4}$-inch focus forms its image at 10 inches, this will be forty times as large as the object $o$. Then if the eye-piece magnifies ten times, the power (with that objective and eyepiece) will be 400 diameters.

12. Spherical Aberration of the Lenses. - If single lenses did perfectly the refracting and image-forming work described in Chapter I, a'microscope would be simple indeed; unfortunately they do not, failing in three ways.

Firstly, they are ground to spherical surfaces, and these refract too much in proportion as we approach the edges; thus the rays falling on the edge of the lens come to a focus nearer the lens than the central rays. The image formed by the large reading glass in $\$ 8$, on close examination will be found rather blurred from this cause, which is called 'spherical aberration.'

Secondly, it will be noticed that print seen through such a glass appears distorted in the marginal parts. And the image on a card of a flat surface like a lantern slide is not itself flat, but curved hollow towards the lens. These are other effects of spherical aberration. 
13. Chromatic Aberration.-Thirdly, in looking through the glass lustre or prism at the candle-flame (Chapter I, § 7) the image was not only refracted, but spread into a band showing all the colours of the rainborv-the coloured band called the spectrum. That is because different colours are differently refracted, blue being the most so and red the least. ${ }^{1}$ This being so, clearly a simple lens must bring the blue rays to a focus nearer to it than the red rays; and this can also be seen, with care, in any large lens. It is called chromatic aberration.

These faults do not prevent much rough useful work with simple lenses, as in a magnifying glass. But they are fatal to precise work, and most of all to the perfection of images magnified many hundreds of times.

14. Correction of Aberrations.-Such defects can be more or less corrected in several ways.

(a) All of them are worst at the edge of a lens. Hence, by stopping all but a portion in the centre, the image is greatly improved in sharpness. But it is at the expense of light and of other functions of large aperture (\$\$ 17, 22). Hence this plan can only be used to some extent, and as the cheapest mode of improvement.

(b) Both aberrations differ greatly in amount, according to the inclination of the surfaces to the rays. The spherical aberration of a plano-convex lens in parallel rays is less than a third when the flat side is turned torvards the focus, of what it is the other way. And two inclinations may be so disposed that the aberration of one largely corrects that of another. Thus, by using two or more lenses, amongst which the power is divided, instead of one, and proper combination of curves, much may be done. This method is largely employed, and is entirely depended upon in the Huygenian eye-piece.

1 The law of proportional sines holds good; but each colour has a somewhat different refractive index $(\$ 5)$. 
(c) Chiefly of all, while different sorts of glass differ largely in both their refractive power (or optical density) and also in their dispersive power (or degree to which the spectrum is spread out by a prism of given angle), the two qualities do not preserve the same proportion. So it is possible to make, let us say; a plano-convex lens (fig. 11) of

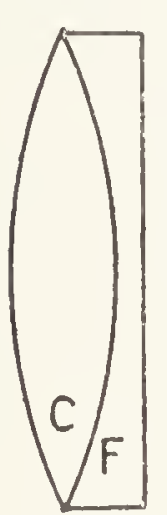
two glasses, of which the crown-glass lens, c, has double the curvature of the flint-glass concave lens, $F$, and so has a large surplus of refractive power, while yet the colour-dispersion of $\mathrm{F}$ is enough to counteract all that of $\mathrm{c}$, and so brings all colours nearly to the same focus. Such a lens is called achromatic, or ' corrected for colour.' Fortunately it also happens that the concave curve of the flint Fra. 1 lens largely corrects the spherical aberration of $\mathrm{c}$ as well; and so we get a more perfect lens every way.

15. Microscope Objectives.-But even such a lens is not generally good enough for the microscope. Though much of the spherical aberration can be so corrected, all of it cannot be, except in the one case where all the rays proceed from one point in the optic axis, and the conjugate focus is at another point on the axis; hence this simple form is much used for astronomical telescopes. More can be corrected by a single triple combination-a form often employed in microscopes for low powers, such as 3-inch, or cheap powers up to 1-inch. But very much more is possible from combinations of two achromatic lenses, separated by an interval, or by additional lenses still. By combining different glasses, with appropriate curves presenting various angles to the rays, the modern microscope objective obtains its excellent results.

It will be pretty evident that very similar results may be produced by quite different combinations. For a half-inch lens of the same aperture ( $\$ 17)$ three opticians may use different glasses, and different combinations and curves; 
then each man's particular lens would be known as his 'formula,' and the number of possible constructions is almost endless - three are shown at D E F in fig. 12, suitable for gradually increasing or higher powers. It is easy to see that some are more complicated, and hence more costly, than others; but that cost may be well spent. On the other
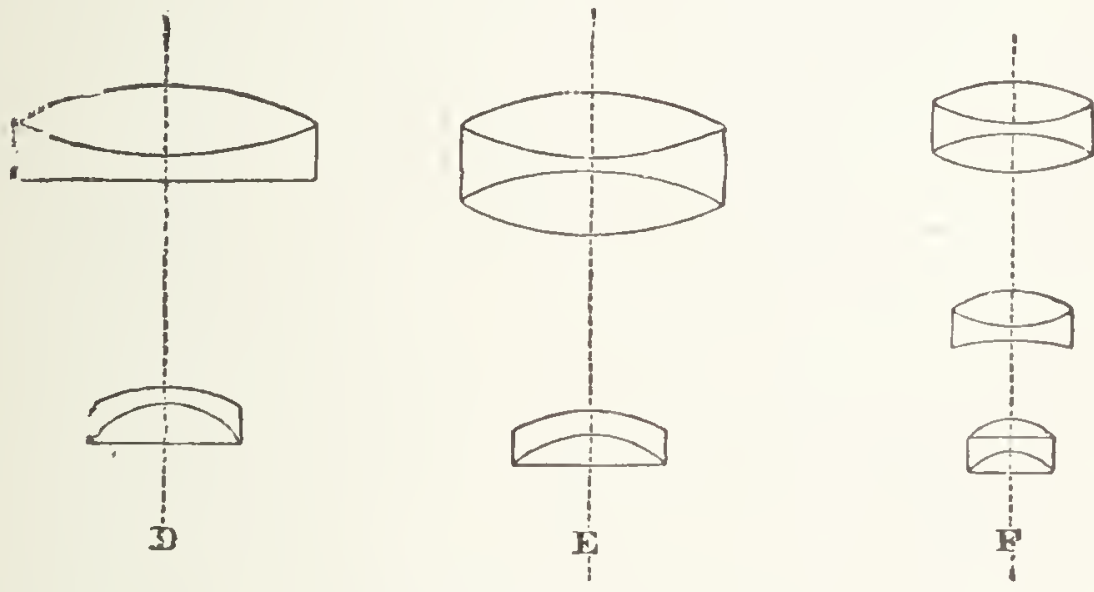

FIr. 12.-Forms of objectives

hand it may not. The best constructor is he who corrects to the utmost, but adds no complication unless it distinctly improves the image. ${ }^{1}$

16. Effect of Cover-glass.-An objective whose spherical aberration is so corrected that all the rays are converged to a sharp and true focus is called aplanatic. If the correction still leaves the focus of its marginal rays shorter than for the central rays ( $\$ 12$ ), it is called 'under-corrected.' If (as is quite possible) the correction is so great that the central rays are actually shorter in focus, it is 'over-corrected.'

As soon as the late Mr. Andrew Ross had constructed good achromatic lenses, he discovered that if one was made aplanatic for an uncovered object, the interposition of a cover-glass upset his corrections. It is easy to see how Let $\mathrm{o}$, in fig. 13, be an object in balsam $\mathrm{B}$ under the coverglass $C$, and $O P P$ and $O Q Q$ two pairs of rays proceeding at large and small angles from $O$. At $P$ P and $Q Q$ they are

The last improvements in lenses are described in $\$ 24$. 
refracted away from the normal, as shown. Producing back these rays as they enter the lens $\mathrm{L}$ by the dotted lines, it is as if one pair really proceeded from $\mathrm{A}$, and the more mar-

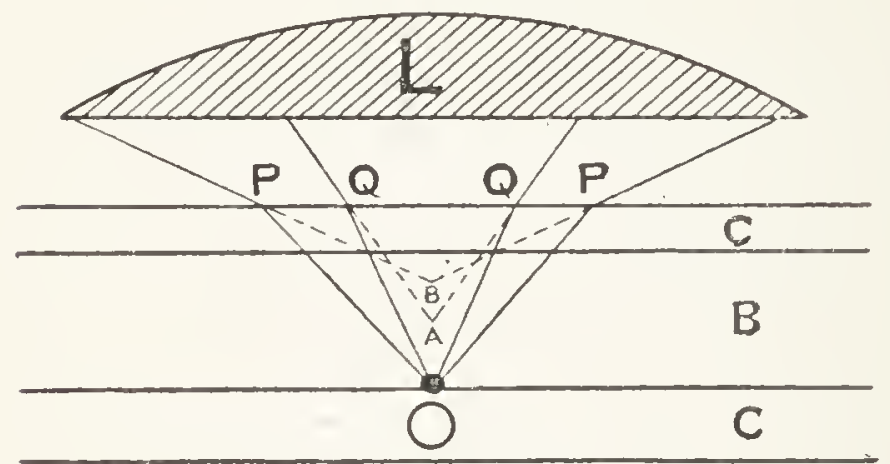

FIG. 13,-Effect of cover-glass ginal rays, $\mathrm{P} P$, as if they proceeded from $B$, nearer the lens. But such foci for marginal and central rays represent undercorrection; and hence the objective, to focus truly under these circumstances, must itself be under-corrected for rays proceeding from o in air. Also, the thicker the cover and balsam above the object, the more of this effect is produced.

Mr. Ross knew that in any objective with two or more parts, properly adjusted, under-correction could be produced by causing the front and back components to approach each other, and over-correction by more separating them. Hence the best high powers, except oil-lenses (see $\$ 20$ ), are furnished with a screw-collar, called the collar-correction, to effect this as required. Under-correction for thicker covers can also be effected by lessening the conjugate focus, or distance between objective and eye-piece; and this method is much used for cheap, and for low or moderate powers. A high-class lens can only be perfectly corrected for one thickness of cover, with one tube-length or focus; and the more perfect the lens, the more sensitive it is to these conditions.

17. Angle and Aperture.-It is plain that two objectives of the same focus may utilise in their images very different cones of rays ( $\$ 8)$ from the object. In fig. 15 the lens takes up a much wider cone of rays $b o b$ from the object $o$ than the similar cone $b o b$ in fig. 14 . These lenses are single, but it is just the same with compound lenses.

The most obvious gain from wide aperture is brightness, 
which in itself may be needed for very high magnifications. But as long ago as $1830 \mathrm{Dr}$. Goring pointed out that there was more than this; and that in examining certain insect scales with minute markings, which he used as tests, wide aperture was so necessary, that even an unachromatised combination of wide aperture had often more of this separating or resolving power, ${ }^{1}$ than achromatic lenses of far better definition, but less 'angle.' This led to gradual increase in the angle of achromatic lenses; with which, however, the aberrations became more difficult to correct, and the working distance also (§ 36) became less. For the greatest theoretical angle of the cone of rays which can enter the front of an ordinary lens is $180^{\circ}$ (§ 6), and such is only possible when the object

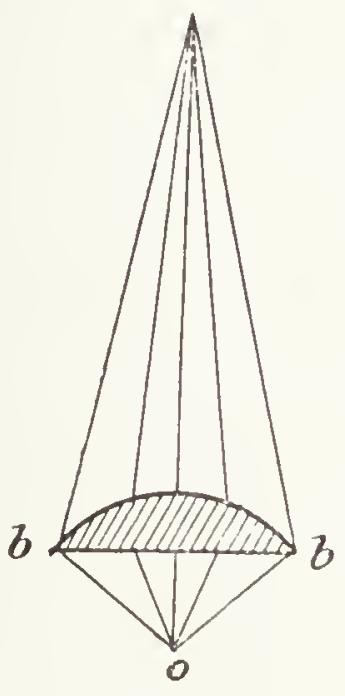

FIG, 14

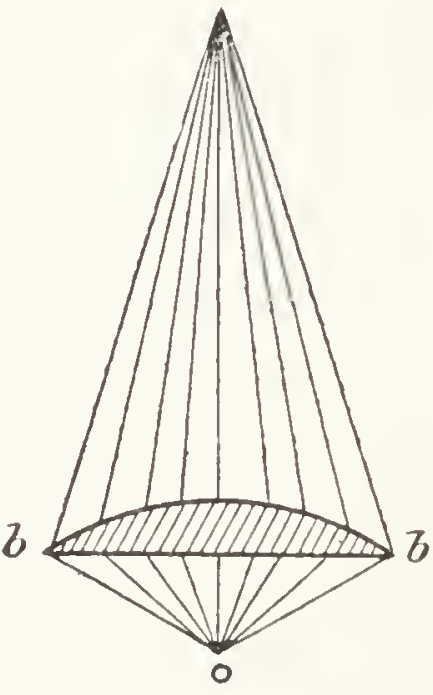

FIG. 15 touches the plane face of the lens, which gives no working distance at all. Even with less angles than this, the loss of light by reflection is (near the edges) very great.

18. Immersion Objectives. - About 1840, Amici proposed to prevent this loss of light by interposing water or oil between the lens and the cover-glass; and he used oil in this way about 1844 . Such a medium, however, quite destroys accurate 'correction' in a lens calculated for air, and needs entire re-computation, which Amici never effected. The idea was, however, taken up again by Hartnack and Prazmowski of Paris, whose high-power 'water immersion'

1 Then called 'penetration,' but now termed 'resolution'; while the term 'penetration' is now applied to depth of focus, or the power of imaging points not in the precise focal plane. 
glasses were found far superior to all others of similar power at the Paris Exhibition of 1867. These lenses were much improved by Powell and Lealand, until about 1877 an angle in water of $144^{\circ}$ was grasped by their lenses. In 1870 the use of oil was again suggested by Mr. Wenham, but only suggested. In $1873 \mathrm{Mr}$. Tolles, of Boston, constructed objectives which used soft balsam as the immersion fluid, and took up an angular cone of $110^{\circ}$ in that medium. This medium is so very disagreeable and difficult to use, that it is nearly certain Tolles must have had a clear apprehension of the essential nature of the connection between greater aperture and greater resolution, outlined in $\$ 22$.

Microscopists owe to Mr. H. Stephenson, however, the suggestion of the final improvement in immersion lenses: viz. the adoption of some fluid which should resemble, both in its refractive index and dispersive power, that of the coverglass and the front lens of the objective. Such a system is termed 'homogeneous' immersion, and its optical advantages were clearly pointed out by Mr. Stephenson. A suitable fluid was systematically searched for by Prof. Abbe, of Jena; and that in universal use now, as the result of such search, is cedar-oil, carefully reduced to the refractive index of about 1.516 .

19. Immersion, Angle, and Aperture.-Amici had evidently no idea of the great gain in angular aperture from the
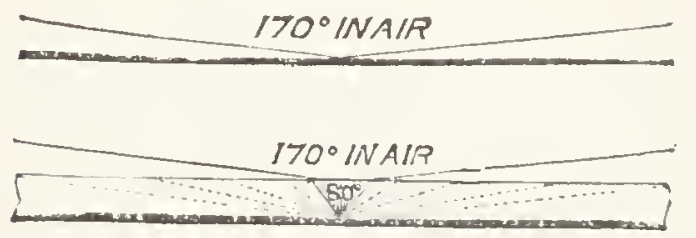

FIG. 16 immersion system ; but this necessarily follows from what was said about the law of refraction, and total reflection, in $\$ 5,6$, and fig. 3 . For in fig. 16 let the upper figure represent a cone of $170^{\circ}$ from an uncovered object on the face of a thin glass - this is the extreme angle (in practice) which can enter' the face of a 'dry' lens. But in the lower figure the object emitting the cone is on the face of a slip 
beneath, and covered by balsam and a thin glass; then, as in $\S 6$, the black cone of $82^{\circ}$ is the utmost angle that can emerge from the balsam, and becomes $170^{\circ}$ in air. The rays outside this, as dotted, are totally reflected back into the slide. But if the space between slide and lens be also filled with balsam, or cedar-oil, there is no refraction, and a far wider cone of the rays in the balsam can enter the lens.

All this is clear gain, and an oil angle of even $110^{\circ}$ is greater than an air angle of $180^{\circ}$ from an uncovered object. For it was established by Clausius, in 1864, that all radiant energy (whether light, heat, or photographic effect), emitted by any substance, increases as the square of the refractive index of the medium. So that if we lived in air that had an index of 1.516 instead of 1.0 , we should 'see' all things brighter in the proportion of $9: 4$. So a glass or oil angle of $82 \frac{1}{4}^{\circ}$ contains all the brightness of the air angle of $180^{\circ}$, and all above $821_{1}^{\circ}$ is clear gain, even in light; while all the 'resolving' power presently mentioned, of greater cones of rays, being similarly compressed in water or oil, increases in the same proportion.

20. Homogeneous Immersion.-Figs. 17 and 18 show the peculiar advantages of homogeneous immersion as pointed out by Mr. Stephenson. Fig. 17 shows a water-immersion lens with an angle of $140^{\circ}$ in water, or $70^{\circ}$ on each side of the axis. This angle is filled by the balsam semi-angle of $56^{\circ}$, as shown, refracted into the water-angle of $140^{\circ}$, which at $a$ is again refracted back to the glass semi-angle of $56^{\circ}$. Thus there is great spherical aberration at this flat surface $a$, before the cone can be further refracted at the back spherical surface into an angle of $70^{\circ}$ to $90^{\circ}$, for entering the back portions of the objective. But with oil, as in fig. 18, there is none of this-there is no refraction at all at $a$; none of any kind till the back spherical surface of the lens, which, presenting a concave curve towards the cone, occasions scarcely any. Thus the actual high angle from $\mathrm{F}$ is reduced to the 
angle of $70^{\circ}$ or $90^{\circ}$ behind the lens (as if the cone procoeded from $\mathrm{F}^{\prime}$ ) without any appreciable spherical aberration, and the difficulty of 'correction' is reduced accordingly.

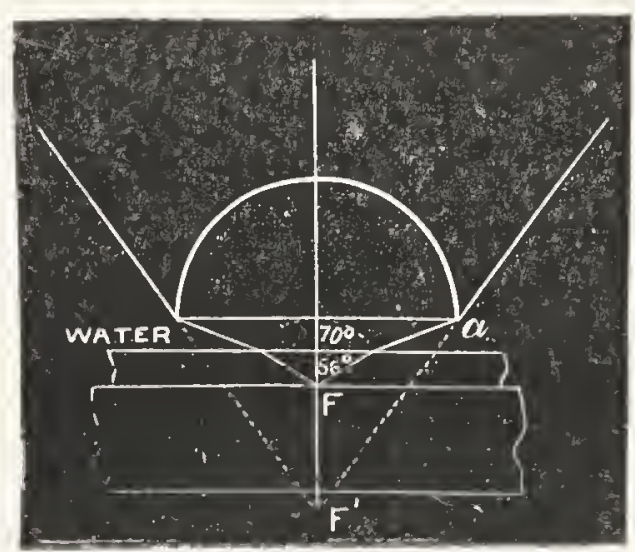

FIG. 17

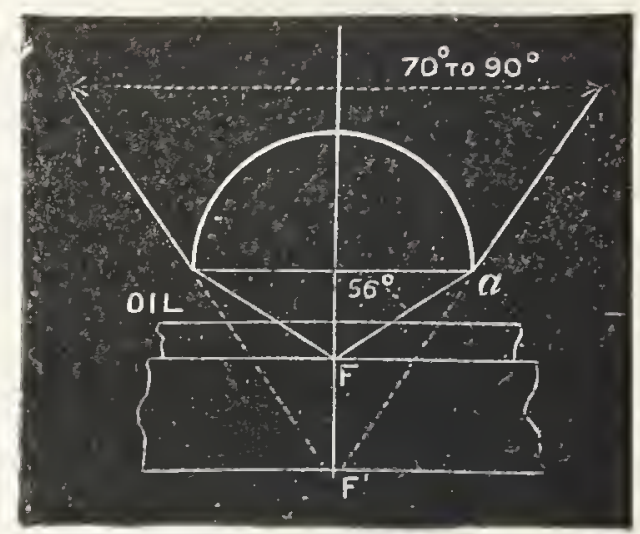

FIG. 18

ù

It will also be seen that there is no need, with this system, of any collar-correction for cover-glass.

For very high-power work, the best lenses are now, therefore, always constructed on the 'homogeneous' system; such lenses being also termed 'oil' lenses. Equal results can be obtained in no other way.

21. Measure of Aperture: Numerical Aperture. - We cannot therefore measure the effective aperture of objectives by angle alone; we must take account of the medium in which the angle of rays is reckoned. The measurement now universally followed is due to Professor Abbe. He showed that, even in lenses for the same medium (as air), their comparative aperture, as compared with their focus, was not correctly measured by the angle of the rays grasped, but by the actual diameters of the pencils of rays transmitted, which depended more upon the back of the lens than its front. To get a geometric measure for comparison, he took the radii, or half diameters (whose relative proportions would be the same), and which, geometrically, are the sines of the semi-angle of the outermost rays grasped. He further showed that, if this sine of half the outside angle 
were multiplied by the refractive index of the medium used, we should have a number which would give us the comparative 'aperture' of any lens, whatever the medium. This number is called 'numerical aperture,' or N. A. Calling the semi-angle $u$, and the refractive index $n$, it is expressed by the formula:

$\operatorname{Sin} u \times n=$ N.A.

The following is a table of some numerical apertures, showing the respective angular pencils which they express in air, water, and cedar-oil or glass. Of the higher powers it is most convenient to take round numbers in N. A., and their angles can only be stated in fluid media; of lower powers it will be most serviceable to give the nearest $\mathrm{N} . \mathrm{A}$. equivalents of the more usual angles used in 'dry' lenses.

\begin{tabular}{|c|c|c|c|}
\hline N. A. & Air angle & Water angle & Oil angle \\
\cline { 1 - 3 } $1 \cdot 40$ & - & - & $134^{\circ} 10^{\prime}$ \\
\hline $1 \cdot 30$ & - & $155^{\circ} 38^{\prime}$ & $117^{\circ} 35^{\prime}$ \\
$1 \cdot 25$ & - & $140^{\circ} 3^{\prime}$ & $110^{\circ} 39^{\prime}$ \\
$1 \cdot 20$ & - & $128^{\circ} 55^{\prime}$ & $104^{\circ} 15^{\prime}$ \\
$1 \cdot 0$ & $180^{\circ}$ & $97^{\circ} 31^{\prime}$ & $82^{\circ} 17^{\prime}$ \\
$0 \cdot 95$ & $143^{\circ} 36^{\prime}$ & $91^{\circ} 10^{\prime}$ & $77^{\circ} 22^{\prime}$ \\
$0 \cdot 87$ & $120^{\circ} 55^{\prime}$ & $81^{\circ} 42^{\prime}$ & $69^{\circ} 49^{\prime}$ \\
$0 \cdot 85$ & $116^{\circ} 25^{\prime}$ & $79^{\circ} 37^{\prime}$ & $68^{\circ}$ \\
$0 \cdot 82$ & $110^{\circ}$ & & \\
$0 \cdot 77$ & $100^{\circ}$ & & \\
$0 \cdot 71$ & $90^{\circ}$ & & \\
$0 \cdot 64$ & $80^{\circ}$ & & \\
0.50 & $60^{\circ}$ & & \\
$0 \cdot 42$ & $50^{\circ}$ & & \\
$0 \cdot 35$ & $40^{\circ}$ & & \\
$0 \cdot 30$ & $35^{\circ}$ & & \\
$0 \cdot 26$ & $30^{\circ}$ & & \\
$0 \cdot 22$ & $25^{\circ}$ & & \\
$0 \cdot 18$ & $20^{\circ}$ & & \\
\hline
\end{tabular}

22. Aperture and Resolution.-It has already been hinted (§ 17) that mere power, or magnification, however well corrected the lens and powerful the light, will not separate 
or 'resolve' markings of a certain minuteness, unless there be a certain aperture as well. A simple experiment will illustrate this. Get a bit of wire gauze with meshes from 50 to 100 per inch, and in a bit of black card prick a small pin-hole, and another pretty large one. With the card close to the eye, look through the small hole at the gauze, and move this gradually away, against the light of the sky. A distance will soon be found at which the separate wires can no longer be seen. Now move the card so as to look through the larger pin-hole (i.e. more aperture to the pupil of the eye) and the meshes appear again quite distinctly.

Space forbids explanation of this in detail, but a general idea of the reason should be grasped. In \$ 3 we took no note of what a ray of light really was. In reality it is simply the propagation of a disturbance in the ether, of the nature of a wave. These waves have wave-lengths, each colour of light having its own wave-length, varying from about the $\frac{1}{400} \frac{1}{00}$ th to the $\frac{1}{67000}$ th of an inch. As it is the convergence of these lines of wave-disturbance that has got to make our image, it is easy to see that when we have to do with very small details, the absolute size of the waves limits the size of the smallest possible image we can get of such a detail. For instance, the waves in a pond have a certain length. If we suppose them set up by a small stone like a marble, much less than one of these wave-lengths in size, we see at once that however such waves might be converged on one point, they could never be reconverged into a space so small as the marble - the waves themselves are too big for this to occur. Now many microscopic details are far less in size than half a wave-length of light; and so this limit comes in. But it can be shown mathematically, and is found in practice, that the wider the aperture of a lens, and consequently the more oblique the outside rays which form the image, the smaller (short of the final absolute limit) the image of a 'point' becomes. This law affects all. lenses, 
from a 36-inch telescope-lens of 20 feet focus, to a $\frac{1}{16}$ oilimmersion objective.

Of course when we have two small points close together, the law determines whether they can be imaged small enough to be separated. It has been calculated ' that 'two lines cannot be fairly resolved unless its components subtend an angle exceeding that subtended by the wave-length of light at a distance equal to the aperture.' An $\frac{1}{s}$-inch dry objective of N. A. 1.0 has a diameter or aperture of $\frac{1}{4}$ inch. If therefore we are using light of a wave-length of $\frac{1}{485}$ of an inch, this at a distance of $\frac{1}{4}$ of an inch (the aperture), will subtend the same angle as two lines $\frac{1}{20410}$ of an inch apart at $\frac{1}{8}$ inch (the focal distance) of the lens-hence this lens will 'resolve' something less than 96,410 lines per inch. ${ }^{2}$ Here, therefore, we find the great gain in optical power of homogeneous-immersion lenses.

This discovery of the function of 'aperture' has revolutionised microscopy. Formerly objectives were made of $\frac{1}{50}$ of an inch focus. These cannot be made of large apertures, and will not therefore exhibit such minute detail as can be easily seen with $\frac{1}{8}$ and $\frac{1}{12}$ oilobjectives of large aperture, with which all modern work is done, and which are far less expensive. Mere 'power' is not of the slightest use, beyond that limit of visual separation which the 'aperture' permits.

23. Diffraction Spectra.-The above law supposes our objective to be filled by an angular cone of rays-else its aperture is not all used. Owing to special difficulties in many objects, and to imperfections in the best objectives and condensers ( $\$ 39$ ), we can, however, scarcely ever use such a

1 Encyclopedia Brit., art. 'Wave Theory.'

2 In the same article Lord Rayleigh shows that the above measure of lesolution is only possible with a square aperture, or one bounded by straight lines parallel to the lines resolved. He calculates that with circular apertures such as those of objectives, the aperture must be increased, or the resolution will be decreased, by about one-tenth. Actual measurements lave verified this as exactly as possible. (Phil. Mag., Aug. 1880.) 
full cone of rays, though advances in this respect are being made $(§ 24)$. Objects displaying a series of equi-distant minute details, however, when illuminated by a narrow pencil or cone of rays, are seen to throw out on each side of that central pencil a series of diffraction-spectra, such as are visible when looking at a distant candle through a feather, or piece of fine cambric. ${ }^{1}$ It is found that, although a much narrower cone of rays be used than the lens will grasp (\$17), if such outside spectra also enter the objective, their rays also unite in the image, and give additional resolving power due to their greater angle. Professor Abbe was the first to study the effect of these spectra, and by using very narrow pencils many curious results and false images were obtained; from which he deduced a theory that interference (and not union) of the rays in the spectra was the essential element in the image of minute structure." Much has been very ignorantly written on this 'Diffraction Theory.' There is no doubt that it has done invaluable work in enforeing the necessity for aperture in all objectives of high power. But many practical errors in it were gradually pointed out; and there can be no real doubt of the underlying truth in it being simply and solely, that the spectra are of service in furnishing rays which utilise the aperture, in cases where the cone

1 These spectra can readily be seen in the microscope by using a narrow pencil of light to illuminate $P$. angulatum, with a $\frac{1}{4}$ inch of $100^{\circ}$ air angle. Removing the eye-piece and looking down the tube, the spectra will be seen surrounding the central pencil, in the back of the objective. It is these spectra which utilise the marginal zone of the objective when a small cone is used (see $\$ 60)$.

2 When a small pencil or illuminating cone is used, marked 'interference' bands and fringes are produced, which in some cases correspond, in mere number of details per inch, with the reality. 'These, however, vary with the slightest alterations of focus, and produce the most marvellous and baffling changes in the appeirances, like all other interference bands and fringes. No one of them can be selected as a true dioptric inage, and so far from being of real use in microscopical study, they are a constant source of false interpretation and error. They are especially liable to double the apparent amount of detail. 
of direct rays which can be employed on an object is insufficient to do so. ${ }^{1}$

24. Apochromatic 0bjectives. - The last great advance in the optics of the microscope is also due to Professor Abbe. Achromatism has been briefly explained ( $\$ 14$ ), but previously to 1881 the best objectives only approximated to colour correction. The colour produced by the convex crown-glass lenses was easily corrected by the contrary and stronger dispersion of the concave flint-glass lenses, so far as regarded the two ends of the spectrum, or any other two coloured rays; but the spectra of the two glasses never corresponded in all rays, and consequently there was always some residual colour left, known as the 'secondary spectrum.' The same discrepancy affected spherical aberration also, so that only one colour could be truly corrected for that. Professor Abbe pointed out in 1876 that removal of these residual errors must depend upon the manufacture of new optical glasses with certain definite properties of refraction and dispersion not then obtainable. In 1881 he and Dr. Schott, aided by a subsidy from the German Government, commenced experiments in conjunction with the optical manufacturing firm of Zeiss, in Jena, and produced various new glasses. Some of these glasses effected high dispersion with low refraction, and some possessed low dispersive power combined with high refractive index; and pairs were produced whose dispersion was in alnost similar relative proportion. With these glasses lenses were calculated which brought to the same focus threc coloured rays, and corrected spherical aberration for two, leaving scarcely any residual error. Such objectives are now made by all the best English and

1 I have discussed this matter at length in The English Mechanic and World of Science, September, October, November, 1894. The conclusions there arrived at have been accepted in all main points by foremost mathematical physicists in this (or any other) country; and substantially by $\mathrm{Mr}$. E. M. Nelson amongst microscopists (see Quekett Club Journal, March 1895 ; 
Continental opticians, and are termed apochromatic. They have enabled results to be obtained in biological research which were previously altogether impossible. Some of the best, optically, of the new glasses were found to oxidise so rapidly on the surface that they had to be discarded; and one or two of those still employed are not so durable as the crowns and flints formerly used; but the optical improvement has been so great, that the highest class of workers would rather, at the worst, have to procure fresh lenses now and then, than go back to the older ones.

Besides new glasses, the best class of apochromatic objectives contain one or more lenses of fluorspar or fluorite. This is not necessary to apochromatism; but its low density and dispersion (little exceeding that of water) enables flatter curves to give the same effect, and so facilitates the spherical correction. ${ }^{1}$ Unhappily clear fluorite is scarce, and the spar is rather difficult to work, which makes such lenses necessarily costly; but the new glasses are used in nearly all modern objectives, and effect far better 'correction' than formerly, even in those objectives falling short of strict apochromatism.

25. Compensating Eye-pieces.-The more perfect correction of objectives made more evident some aberrations in those with thick plano-convex fronts (including all high powers) as regards the margin of the field, which were almost beyond correction in the objective itself. These also Professor Abbe got rid of by contrary 'over-correction' in the eye-piece ; the plano-convex ocular lens of a Huygenian eye-piece being replaced by an achromatic with rather too much flint correction. Such 'compensating' eye-pieces so greatly improved all high powers, that corresponding aberration was purposely introduced into the lower apochromatic objectives also, in order that the same eye-pieces might be used throughout.

$1 \mathrm{Mr}$. Spencer used fluorite in America in 1860. 
The result of these modern discoveries and improvements -chiefly due to Professor Abbe of Jena-has been to abolish the terribly expensive and very high powers of former days. It has been shown that all the 'resolution' possible can be obtained with one-eighth or one-twelfth inch oil immersions ; and that such perfect images can be obtained with these, as will bear an amount of further eye-piece magnification formerly unknown. In all the older works on microscopy, great magnifying power is recommended to be got by highpower objectives and low-power eye-pieces. Now, the compensating eye-piece in most general use magnifies twelve times, and a power of twenty-seven times is used very frequently. I have seen the phenomena of cyclosis in vallisneria ( $\$ 114$ ) shown by Mr. T. H. Powell most beautifully, sharp and clear beyond criticism, under a magnification of $\times 1,000$, of which $\times 25$ was obtained by a $\frac{4}{10}$-inch apochromatic, and $\times 40$ by a quarter-inch eyepiece. Such a proportion in the respective magnifications, and such definition of such an object under such a high power, would have been incredible to any microscopist so lately as 1880.

\section{CHAPTER III}

THE SIMPLE MICROSCOPE AND ITS USE

The optical action of a simple microscope was explained in \$ 8. Here we have only to deal briefly with its forms and its practical use.

26. Single and Compound Lenses.-Single lenses are constantly used by engravers and others, but the well-known eye-cell which these employ is only suitable for their work and with low powers. For general use the most convenient 
plan is to have the lens or lenses in an ebonite or horn mount, into which the glasses fold back. Such can be bought in great variety. A useful form in some respects is shown

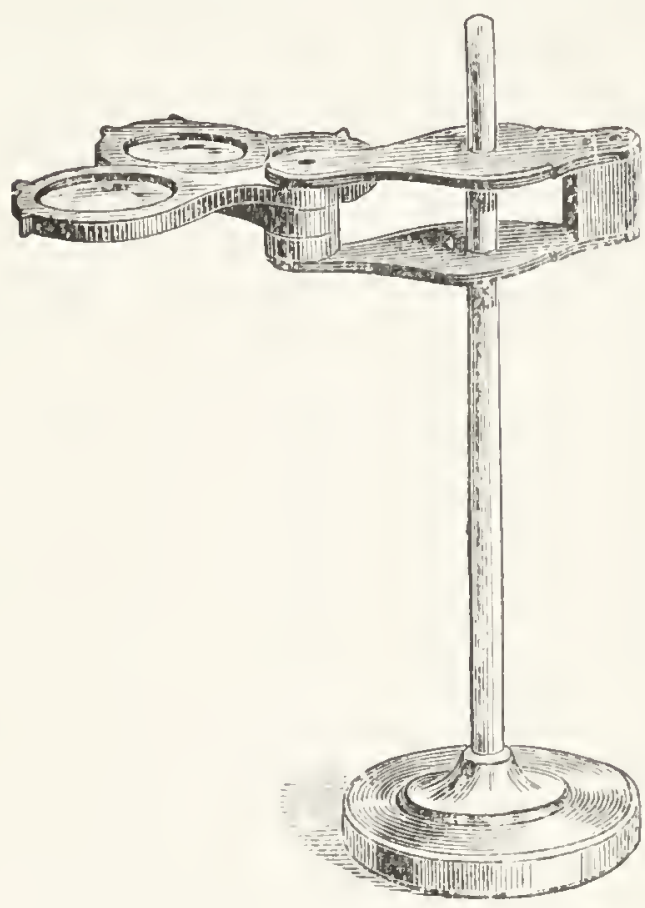

FIG. 19 in fig. 19, where two lenses are shown thus mounted. In the handle or case end a hole is bored, by which the lens can be fixed on a slightly tapered rod in a stand for holding the lens when both hands are needed for other work. Such a double lens may cost about $2 s .6 d$, and the stand $2 s$. The hole in the handle, however, allows dust to get to the lenses. About the most generally useful powers for the two will be 2 inches and $1_{\frac{1}{x}}^{\frac{1}{x}}$ inch focus, which gives three nicely graduated powers. A single lens in a handle can often be got for $1 s$.

Higher powers are smaller, and are often fitted up two at each end of one handle. Two modifications of the simple lens are also made for rather high powers, known as the Stanhope lens and the Coddington lens. The latter is a

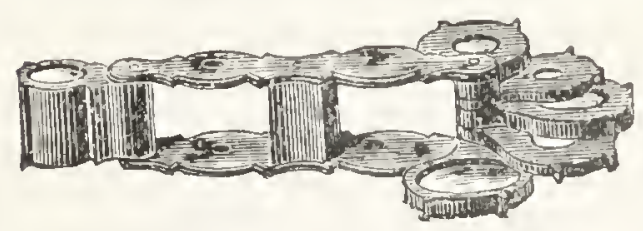

FIG. 20 spherical lens, which has a deep groove ground out all round its diameter and filled with black material, so that light only passes through a small aperture at the centre of the sphere. Fig. 20 shows a Coddington lens in one end of a mount, and three plain lenses at the other, with two stops that can be used between them. The Coddington lens is hardly worth its cost, however. 
The Stanhope lens is really a short cylinder of glass with spherical surfaces at the two ends, the cylinder being such a length that the farthest spherical surface is in focus. This lens also is of no utility except for the one sole purpose of examining rotifers, diatoms, or other minute objects in a drop of water. For this it is handy, a drop being placed on the lens itself, and being in focus when the eye is applied to the other end of the lens.

The best lens of all is an achromatic form introduced by Steinheil, and known by his name. It is a triple combination of the form shown in fig. 21, and gives beautiful definition, with a wide and flat field. Mounted to fold in a handle, these are known on the Continent as Steinheil loups (fig. 22),

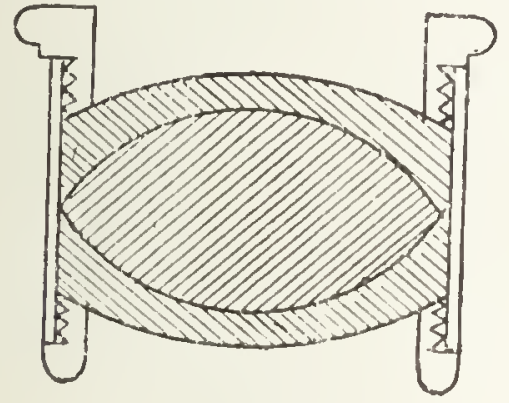

F11. 21

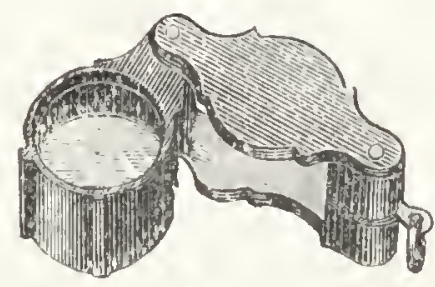

FIG. 22

and can be obtained of any optician for 14s. to 20s. each, of foci varying from 2 inches $(\times 5)$ to $\frac{1}{2}$ inch $(\times 20)$. Only quite lately these beautiful lenses have been made by Leitz of Wetzlar at the low price of 10 s. each, either in a handle, or in a plain collar for use in the dissecting microscope shown farther on. The best plan is to combine both uses, and have two or three powers in collars, with a spring ring folding into a handle, which will carry any one of them in that manner. A Steinheil lens at this low price costs little more than a Coddington, while its performance is infinitely superior. Browning's Platyscopic lens is also a good pocket magnifier.

27. Dissecting Microscopes.--If a single lens is so mounted that it can be focussed by a rack and pinion, over 
a convenient stage on which the slide or object can be placed, and with a mirror underneath which moves freely all ways so as to throw light upon the object through an aperture in the stage, we have converted the single magnifying glass into a real instrument, with which real work can be done. Well made, with a firm stage and side rests attached to the

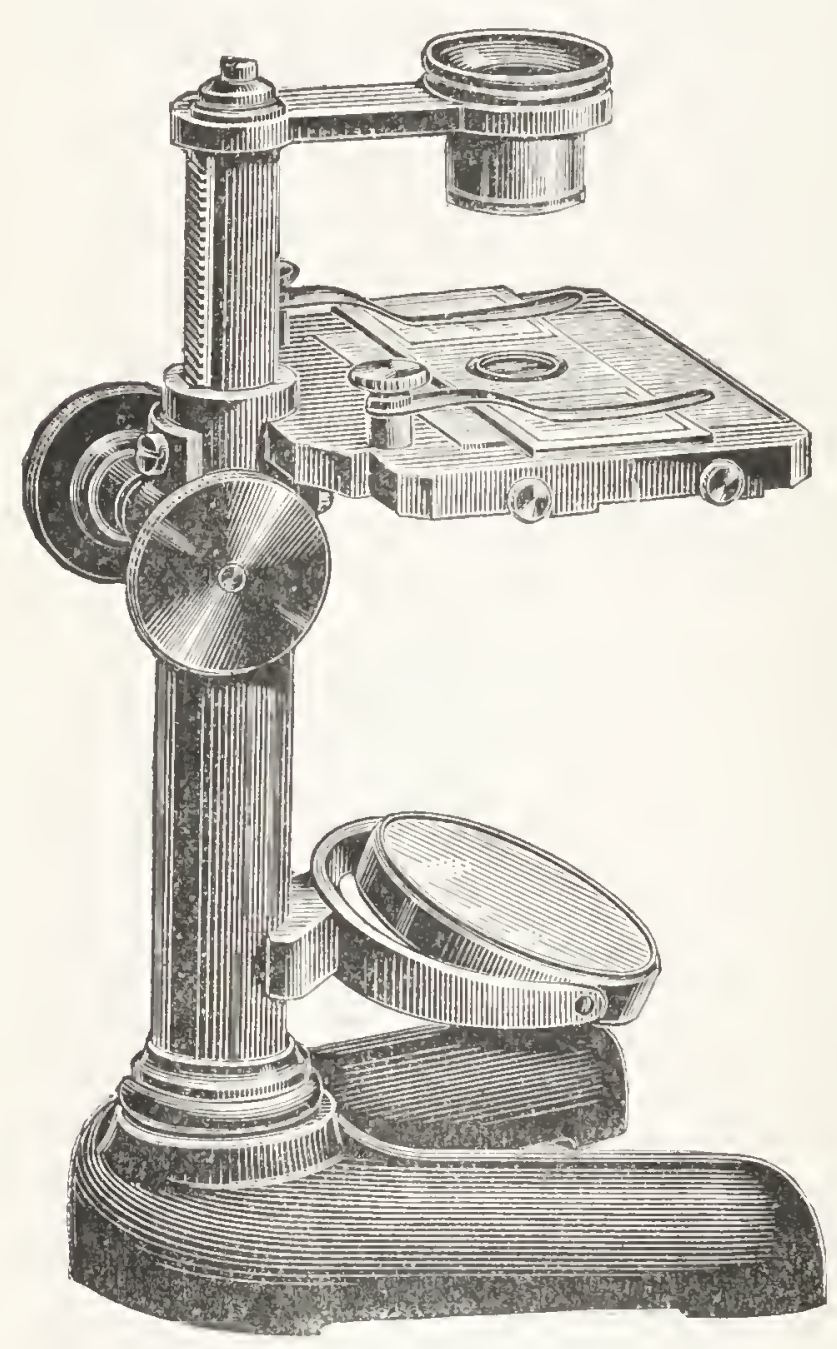

FIG. 23.-Dissecting Mieroscope stage, to support and steady the two wrists, this becomes what is generally known as a dissecting microscope. The best combination of cheapness with efficiency in such an instrument which I have been able to find after extensive and systematic search, is Leitz's, shown in fig. 23. The side-rests for the hands or wrists on each side of the stage are detachable, and not shown in the figure. There is a very good stage and mirror and rack motion; and the instrument is sold, with two achromatic Steinheil lenses (of either focus preferred), for the sum of $38 \mathrm{~s}$. To a real working microscopist such an instrument is invaluable, and will be in use for a lifetime.

28. Use of the Simple Microscope.-No microscopist who has the root of the matter in him ever goes out without a pocket lens of some kind; and a person ignorant of its use has no idea of what can be seen with it, or of the additional 
interest it is capable of imparting to the world of nature around him. Did I wish to awaken in a young person of either sex such an interest in that world as might probably make the microscope a means of enjoyment and improvement for life, my first present would not be a compound instrument, which might very probably be 'played with' a few times and then tired of, but rather a good pocket lens, with a little practical teaching of what could be seen with it during any walk in the fields.

Take it, for instance, as a friend who will reveal countless hidden beauties in flowers. First, use it to examine the very smallest, which to the naked eye exhibit practically nothing: such as the tiny white florets of fool's parsley, wild carrots, and others of similar magnitude, not forgetting in the season the exquisite 'flowering' on 'tail' and other grasses. It is wonderful at first to see how, under' a simple magnifier, exquisite lilies start into being. Examine also the parts of larger 'composite' flowers; see how each so-called 'petal' of a daisy or feverfew is in truth a perfect little floret by itself; how a head of clover is 'made'; a thistle flower'; and others of that sort. Then let larger objects have attention; it will be marvellous to see the added beauties which appear in such familiar flowers as the forget-me-not, pimpernel, agrimony, heaths of all sorts, valerian, sainfoin; and even in flowers so large as lily of the valley, the hairy yellow St. John's wort, or the various forms of orchis. This is no attempt at a list; let the owner of a pocket lens, during one walk, simply make a point of looking at every smallish flower or grass in flower that he can find. The small flower thus magnified is not merely like a larger one; there will be seen an exquisite beauty and waxy texture, in most, that only the finest lilies resemble on a larger scale; and many a common flower-the hedge stachys is another good example-puts on a magnificence of decoration absolutely enchanting. 
Then the reproductive organs of larger blossoms will repay examination. Though but a portion can be seen of the beauties of anthers, stigmas, and pollen grains, enough will be glimpsed to awaken interest and curiosity to know more; and it is such living interest, and desire to look into things, which makes the real microscopist. Yet another world of interest will begin to open as various mosses and lichens are examined; and something of the lovely miniature forest contained in a spot of 'mould' may be understood, even from a pocket lens.

A second class of objects likely to interest an intelligent person will be found in the insect world. Tiny insects no larger than a pin's head, only specks to the unaided eye, grow into wonder and beauty under the lens. Little iridescent beetles and flies will exhibit a magnificent wealth of colour and ornament never imagined. With the higher powers, the curious variety of insect organs can be made out, and will add tremendous interest to any popular book on the subject. The heads of insects alone, with their many organs, so totally different from those of all other animals, will furnish interesting study for months. They are easily examined by preparing a few cones of gummed paper wrapped round and round, and trimmed evenly at the ends, of various

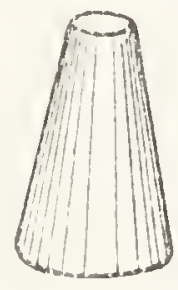

FIG. 21 sizes, as fig. 24. The insect being inserted head first in one of suitable size, so that it can get its head outside, and no more, a little tuft of cottonwool is put in behind and held by the end of a finger, when the head can be examined comfortably. For weevils and beetles the cone is first pinched oval, or nearly flat, so as to fit roughly the section of the body. From personal experience, I can affirm that even experienced microscopists, previously unfamiliar with it, have found absorbing interest in this method of examination, which is within the reach of any boy with a shilling magnifier. Then the insects, killed as usual in a chloroform 
or laurel bottle, may be cut up and their organs examined in detail.

Something can be seen with a pocket lens even of microscopic pond-life, but this mostly requires more or less training both of eye and search (see Chapter VIII). Such is not, however, the case with sea-life, and the lens will add great interest to a sea-side holiday. Take a few glass tubes, with corks, and in these place for examination, with clean seawater, bits of weed or rock that seem to have anything on them different from the mere weed, especially anything in the shape of slender white or creamy branches, no thicker than small or large pins. After a gale, even at places where there are no rocks, such can be found in great variety on weed washed up by the waves; and in the tube, under the lens, they will be seen to be lovely Hydrozoa. Abundance of small crustaceans, and curious sea-worms, \&c., will also be found, which cannot be detailed here.

Something might be added about examining even bits of stones, fresh broken; a bit of granite, for instance, will show plainly how complex is its structure. Seeds, spores, insect eggs, \&c., may also receive attention. But more is unnecessary. It is only desired here to indicate how to use a simple microscope. Anyone who has so used it, more or less constantly, through one summer season, will have a far better idea of the world of minute nature, a truer conception of its wonders, and most of all, a more vivid idea of how much there is to repay further examination and study, than many an one who has had presented to him a costly instrument and box of objects, and who having looked over those objects, has perhaps laid the whole aside. He may probably have learnt to value the microscope for what it really is, and be fairly on the way to become a microscopist.

The use of the simple microscope in dissecting belongs chiefly to a later stage. 


\section{CHAPTER IV \\ THE COMPOUND MICROSCOPE AND ACCESSORIES}

Grent changes have taken place in the microscope since the late Rev. J. G. Wood wrote that a first-rate one cost 'forty or fifty pounds and upwards,' and recommended, as all that could be desired, a three-guinea instrument-' not half the price of a single object-glass belonging to the larger microscopes.' From about the year 1880 especially, development began to settle into one definite line; the result of which, with the improvement in lenses already sketched in Chapter II, has been great economy, combined with so much greater excellence, that better results can now be accomplished with 15l. or 207. than with the 50l. above quoted in former years. Even the very cheapest instruments have shared in this progress; but the greatest gains have been in those of a medium quality. Nearly all the old patterns have disappeared, replaced by new ones, which at first sight seem so various, that a novice may not perceive the one general line of development, unless it is pointed out to him.

29. The Modern Microscope. - Comparing older and modern stands, the modern stand of any good maker provides a steadier 'fine adjustment,' wherewith to precisely focus the much better lenses now used; in fact, the entire microscope is steadier than the bulk of older instruments. But chiefly, whilst in the older text-books all sorts of subsidiary apparatus, such as spot-lenses, paraboloids, and various 'illuminators' and devices for using oblique light are mentioned; the modern instrument supersedes all these by focussing on the object an image of the light, by a lens or combination of lenses under the stage called the substage condenser. In the cheapest instruments this slides into a 
simple tube-fitting under the stage; but in really good ones the condenser lenses fit into a ring, which can be focussed up and down by a rack and pinion, and centered by two screws. A proper condenser thus mounted does all that is required (for transparent objects) far more efficiently, and the best results cannot be obtained without it.

30. Choice of a Microscope.-The best microscopes are made by a comparatively few London houses, who are in constant touch with the two principal microscopical societies, and keep up with the march of improvement. The same real value or cheapness cannot be obtained elsewhere. ${ }^{1}$

The advice of a friend who really is experienced is valuable; but some profess knowledge on very small foundation. It is also to be regretted that even acknowledged authorities, of high reputation, have on various occasions enforced as positive canons what are neither more nor less than dogmatic prejudices, likely to prevent many persons from obtaining what to them would be the best value for their money. ${ }^{2}$ Some details of many stands have weak

1 Many other paragraphs will show, it is hoped, total absence of any antiGerman prejudice; but in face of the persistent pushing of German stands by professors in colleges, it is well to state that these are neither better nor cheaper than English. No doubt it has been largely done in simple ignorance of practical microscopy, though when it is carried so far as to direct students by notice to procure certain stands, 'which can be obtained of - -,' it is difficult sometimes not to suspect other reasons. I was personally informed of a case in which a student, having previously purchased an excellent stand with a substage, and declining to buy another for the study of Bacteriology, the Profcssor manifested the greatest irritation, and finally told him he ought to take it back, and insist on exchanging it for one without that invaluable appliance, demanding the difference in price! Without discussing motive, or giving any clue which can cause pain, when such things are done to the at tempted prejudice of English makers of the highest class, it is time to allude to them and state the facts. German makers are only now adopting accepted improvements in which England has always led the way; and though some of their stands are now very good, for equal work they are quite as costly. Excellent stands and objectives are made in America, but are much more expensive.

2 Three examples may be given. 1. I remember that at a certain meeting of the Royal Microscopical Society a certain maker's lever fine adjustment 
points; but every detail in extensive use has some special advantage which has gained it that position, and which a wider knowledge and more dispassionate judgment would have recognised.

Leaving specific details and stands for the moment, one or two points should be looked to in choosing any stand. That of any good maker will have scarcely any grease about its sliding parts: much should arouse suspicion. The stage should be firm. Chief of all, however, both the coarse and fine adjustments (see next section) should work with perfect delicacy and steadiness, without any play or back-lash, and yet without necting force. Particularly, if the rack-work of either the focussing motion, or of the substage, needs force to move it, any such stand should be refused, as should any which gives a feeling of jerkiness or grittiness in work. The slightest motion of finger and thumb should turn the milled heads, else precise focussing cannot be done; and a good microscope (even at five guineas) should focus a 'dry' $\frac{1}{4}$ or $\frac{1}{6}$ easily. The vendor may say that a tight or hard rack will 'wear easy.' It has no business to do so ; and the process means a wear which is destructive, and ruinous to good work.

Lastly, up to a certain point get the best stand you can, if only with two good objectives and one eye-piece. Keep in mind, if possible, the addition of any important extras, as small additional sums may be afforded, which cannot be afforded at first. Chief among these regard a focussing and

was most unmereifully eondemned as bad by a leading authority. This same adjustment is now generally admitted to be one of the best. 2. The horseshoe foot has been condemned in the most unqualified terms; for large classes of practical workcrs it is the most convenient and useful. It sells more largely than almost any other; and this would not be without reason. 3. The micrometer serew adjustment has been similarly condemned, as ' wearing out.' I lave known it do so when badly made. But this form of adjustment has done more than any other to eheapen good instruments; I have eome across several whieh lave been in constant use for years without wearing out; and if it does wear out the cost of renewal is only a few shillings. It is not the best; but such considerations are not to be lost sight of. 
centering substage. In the old days the first 'extra' added to other than the cheapest microscopes was a 'mechanical stage,' which moves the slide about by screw motions. Many such microscopes are made still by anonymous wholesale houses, for country sale. Some of the best working microscopists think little of a mechanical stage at best, and some actually prefer a plain stage; but not one such will dispense with the substage if he can possibly afford one, and the additional cost is only about $2 l$.

31. Parts of a Microscope.-Let us now briefly consider the essential parts of a microscope stand, from a lettered drawing (fig. 25) of one referred to in the next section, in which section various stands suitable for given purposes or expenditure will be found.

The Foot (F) is the base on which the whole stands. Its best form, for mere working and steadiness, is a wide 'tripod,' as in figs. 33, 34. This form was first carried out in Powell $\&$ Lealand's best stands, and is now widely made; but it has the drawback of requiring the largest and heaviest case. It is the best for constant critical work upon the table. The foot shown in the figure is the 'claw' foot, also a kind of tripod, but covering a smaller triangle, and hence less steady. On that very account it is more handy for packing into the case, or standing under a shade, and is very widely used. The most portable form of all was first used on the Continent, and is known as the 'horse-shoe' foot (fig. 30). As often made it is far from steady; but a rather larger scale than formerly is now usual, assisted by a spur at the back of the horse-shoe, as shown in figs. 30, 31. Such feet are steady enough for all ordinary practical purposes, even without the additional stability given by the reversing pillar described on p. 57; and the smallness of the case which will contain a stand of this kind, and the rapidity with which it is packed or set up, make it the most handy form for medical students and others who constantly carry their instruments about. 
Stands are also made, as Messi's. Beck's 'Economic' and 'Pathological' models, supported on a central pillar from a true flat tripod foot; and the 'four-legged' microscope

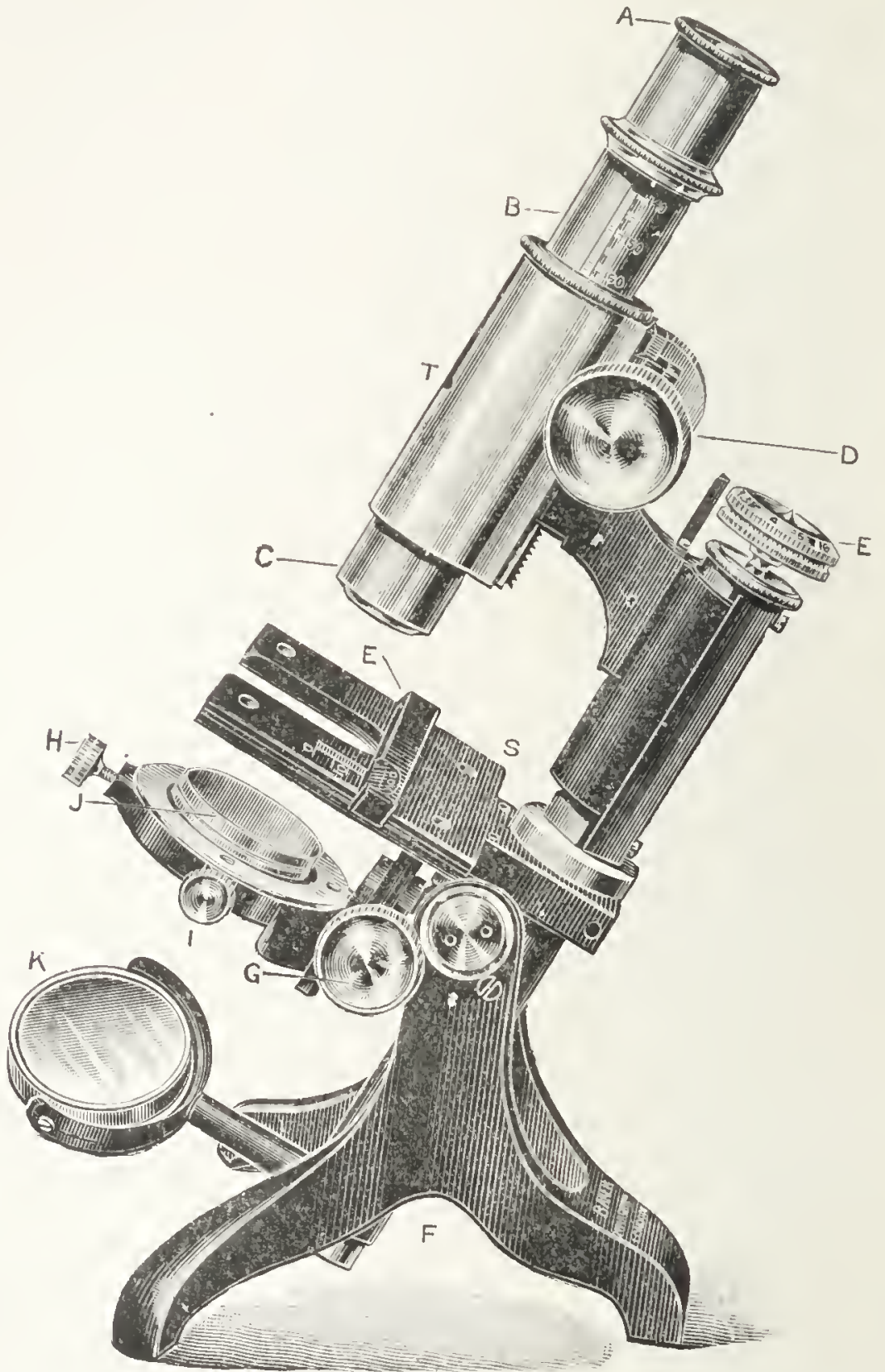

FIG. 25.-Parts of a Microscope

shown in fig. 32 is a modification of the tripod form. A double-pillar on a tripod foot is also used.

The Stage (s) is for the support and manipulation of the slide or other object. It must be pierced in the centre for 
illumination from below, and the aperture should not be less than an inch in diameter. It is better more, and a convenient form for simple stands is that shown here and in fig. 32, suggested by Mr. Nelson, and called a horse-shoe stage, the opening being carried out to the further edge. A plain stage may be fitted with two plain springs, as in fig. 28; but it is much better as shown here, with a 'sliding bar' $\mathrm{E}$ carrying springs which can be turned aside. The great advantage of this is, that by pushing the slide along the bar every point in the slide can be systematically examined (\$50). Another: great advantage is in using high power's (p. 90).

The sliding carrier with two handles, seen on some stages, I regard as useless; and a mechanical stage is only needed by few. For study of minerals, and of other objects by polarised light ( $\$ 40)$ a rotating circular stage is useful, which may be either quite plain or combined with a mechanical stage.

The Tube or Body (т) carries and connects the lenses of the microscope. It is of variable length, Continental objectives being corrected for a focal length of about $6 \frac{1}{2}$ inches, and some best English objectives to one of 10 inches; of late, however, most cheaper lenses and instruments are made in England also for $6 \frac{1}{2}$ inches. ${ }^{1}$ As microscopists wish to use such lenses as they prefer, it is usual in all but the cheapest stands to have a sliding draw-tube (B), or in the best stands even a double draw-tube, so that all lengths can be obtained. The bottom of the tube has a screw at c (called the 'society screw,' and adopted as a standard by every maker), into which the objectives screw; and into the top of the draw-

1 Messrs. Zeiss have done an ill service to general microscopy in adopting the mechanical 'long' tube-length of 10 inches. That was not the average English length, which was about $8 \frac{1}{2}$ inches; and this latter would have been far better and more convenient for obtaining both by one draw-tube. Moreover rearly all good objectives perform better when adjusted or corrected for the longer length, besides giving higher power; and it is distinctly to be regretted that such length has been so heavily discouraged. 
tube fits the eye-piece A. The eye-pieces should fit in quite easily, and not have to be pushed, or hardly at all.

The coarse adjustment (D) is by rack-work. Some very cheap stands are made with only a sliding movement; but there can be no pleasure or even comfort in the use of such an instrument. As already hinted, the rack-work should work smoothly and easily enough, to focus accurately a 'dry' $\frac{1}{6}$ objective.

The fune adjustment (E) is made most variously of all the points in a modern microscope. That preferred by Dr. Dallinger, and used in Messis. Powell \& Lealand's best stands, is in a much rougher form shown in fig. 27, the screw acting on the end of a lever of the first order, the pivot of which is seen further along the hollowed bar, while the other end moves a nose-piece only, with the objective, inside the body. The same movement, but with a longer arm to the lever, and lifting the entire body, is Messrs. Watson's adjustment. Swift's (wherever the direct screw is not used) is essentially the same, but with the long arm of the lever bent down at right angles, parallel with the body-tube. Figs. 30 and 31 show a direct-acting micrometer screw, which is general in all Continental stands; and our present figure (25) shows a double or 'differential' screw, acting on the body in the same general way, but with the advantage of securing a finer motion with much coarser threads, as only the difference between the two screws is effective.

As already hinted (p. 46, note) I consider the condemnation passed upon all these later forms (i.e. those moving the entire body) by a high authority, uncalled for. The disastrous wear predicted, as a matter of fact does not take place in well-made instruments (I have personally examined several which have had long and really hard wear). Hasty dicta of this kind are to be regretted, as condemning the very methods to which we owe such excellent instruments at such moderate prices. Friction is strictly calculable, and is not in- 
creased to nearly the extent supposed by a ferw ounces more weight, if fitting is really good; and by some strange mistake it has been overlooked by one writer, that instead of the pressure of the spring giving additional friction, the weight may be balanced by the spring as nearly as desired, and generally is so. Moreover, the screw portion can, if necessary, be renewed for a very few shillings; or its wear may be compensated, as in Swift's stand (fig. 32). A good differential screw is undoubtedly the best form of direct screw adjustment, and costs a few shillings more than the plain screw.

There is one antiquated form of fine adjustment which it is so important should not be purchased, that it is here represented (fig. 26). A screw working in a bracket fixed on the outer tube, moves an inner tube bearing the objective, sliding in the outer tube. There are hundreds of stands in shops (especially provincial shops) with this, and quite recently I saw a $20 l$. binocular with mechanical stage, so fitted. But it is to be absolutely rejected, whether the screw be at the back as here shown, or in the front, or at the side. It cannot focus anything steadily, better than those cheap dividing objectives sold with the very cheapest microscopes; and

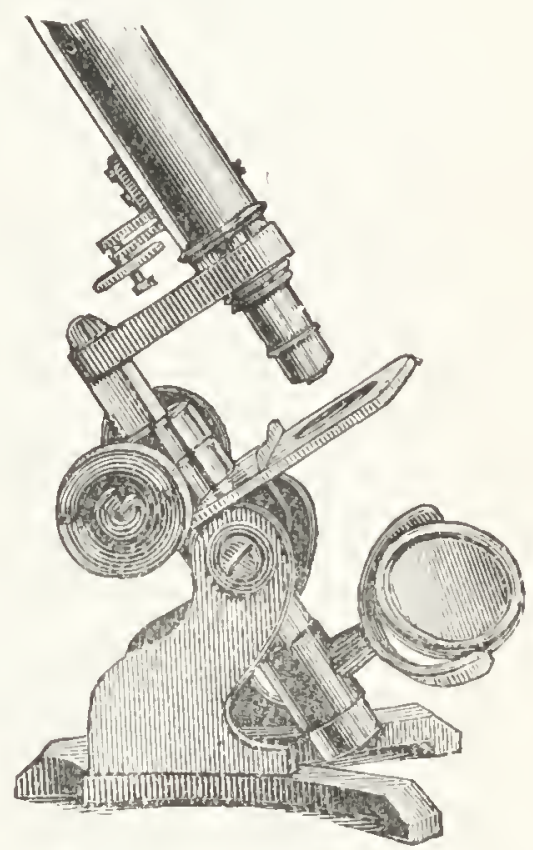

FIG. 26 is discarded by every house of the slightest reputation at the present day. Its utter supersession was the great work done by the micrometer movement.

The mirror ( $\mathrm{K}$ ) is flat on one side and concave on the other. If possible a stand should be chosen with a mirror of good size.

The substage (J) has already been referred to. Only the cheapest instruments have nothing of this kind. A really 
efficient microscope has the substage tube (which also is a standard gauge of $1 \frac{1}{2}$ inch in all instruments) capable of being centred by two screws $\mathrm{H}$ and $\mathrm{I}$ acting at right angles, and focussed by a rack and the milled head G. Such is described in catalogues as a ' focussing and centering substage.' A plain spring tube of the same gauge is screwed to the

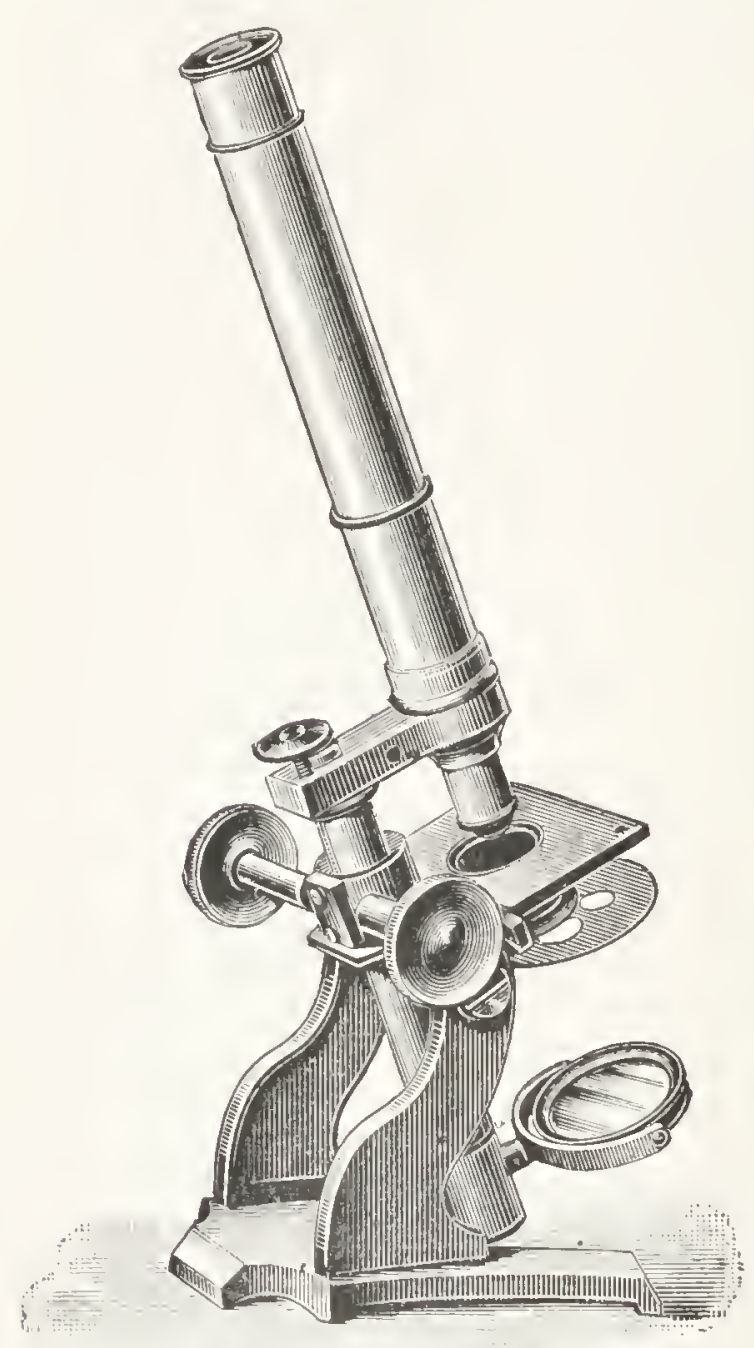

FiG. 27 bottom of the better cheap microscopes. A fairly good focussing and centering appliance costs about 38 s., and centering movements only are supplied by Messrs. Watson for 10 s.

\section{Microscope Stands.}

We will now look at some representative stands at various prices, beginning with the cheapest. ${ }^{1}$

In 1855 the Society of Arts offered a prize for the best microscope that could be made for three guineas, which was won by Messi's. Field \& Co., of Birmingham. The pattern has been made since by many opticians; but I think their own make, shown in fig. 27 , still the best, as having the best fine adjustment of those I have met with, and having two objectives, a separate $1 \frac{1}{2}$-inch, and a $\frac{1}{4}$-inch which separates

${ }^{1}$ All the instruments named are personally known to me, and I have made a thoronghly catholic thougl typical selection. Obviously a complete list is quite impossible in the space at disposal; and many instruments, including the whole made by Powell \& Lealand, are omitted as being too expensive for most of the readers here in view. 
so as to make a $\frac{1}{2}$-inch. Other makes have generally one lens separating into three, and the lowest power is in these cases the worst of all. The instrument has two eyepieces, a wheel of diaphragms, and stage forceps, and is packed in a case with a stand condenser or bull's-eye.

Such a type of instrument will do a great deal, showing nearly all the objects named in most popular works. It has introduced many into this fascinating region of study, and is able to reveal a great part of those wonderful works of the Creator which the microscope only can display. Where it fails to justify such high praise as the writer already named formerly bestowed upon it, is in show-

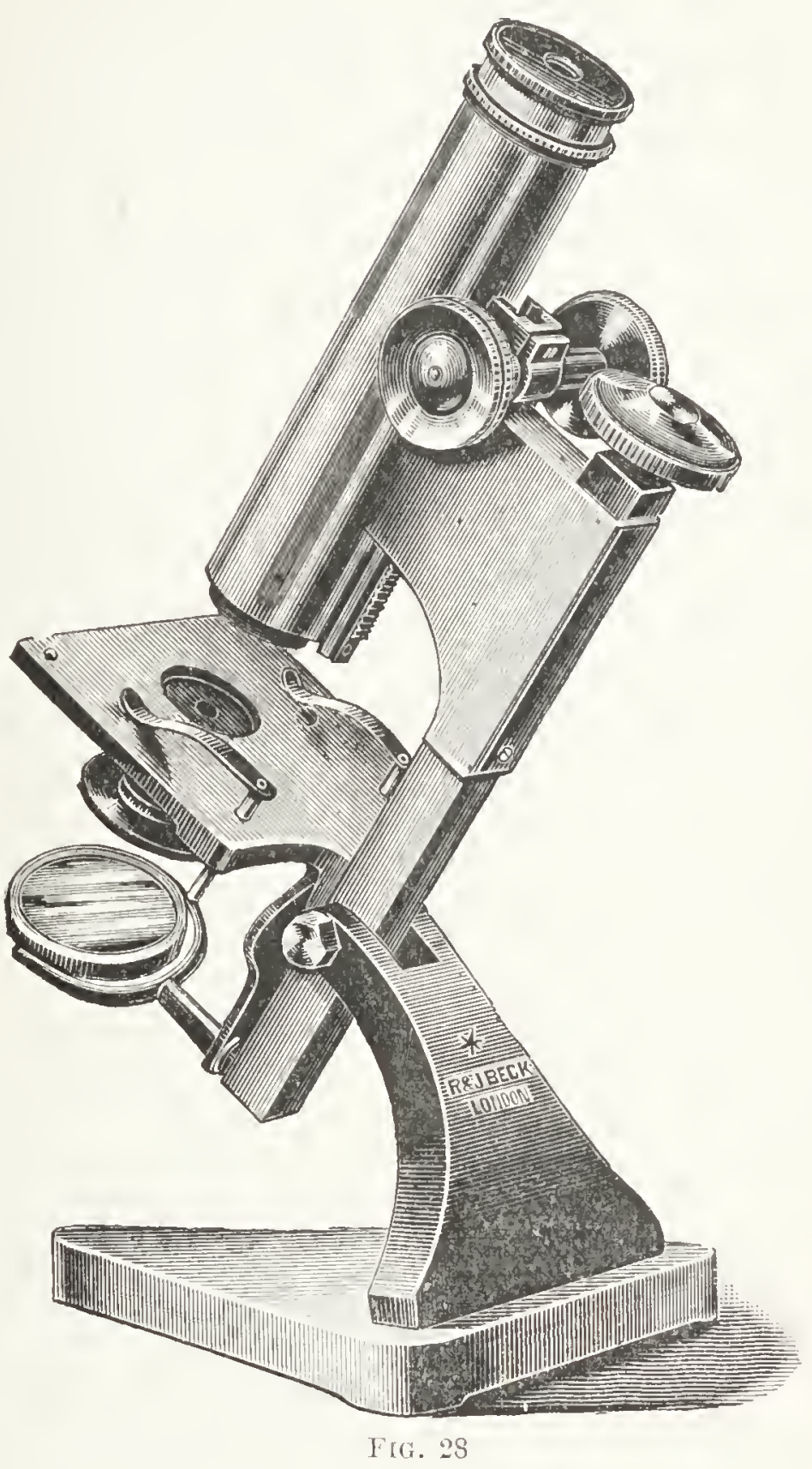
ing the exquisite delicacy of those works in thcir minuter details. The details often appear, and are 'magnified' and made amply apparent; but what appears a thick and coarse cord under objectives of this class, may probably become a filament or line of inconceivable fineness under better powers. 
Let us next suppose that real work is intended from the first, but that only a very little more can be afforded; what is the best that can be done? Probably one of Beck's 'Star' microscopes. This will not be so nice-looking on the mantelpiece as even the preceding, everything being spent upon working parts, and not a penny upon bright brass or outer finish. It can even be obtained for three guineas, in a cheap case with two eye-pieces and one objective-an inch. For another guinea a quarter-inch will be added, or if the purchaser be a medical student it will be better to have two powers, of $\frac{2}{3}$ and $\frac{1}{6}$, instead, at a little more. What is gained will be a steadier fine adjustment, two much better objectives, an iris or contracting diaphragm, and the power to add in the fitting of the latter, for another 10s. 6 ., a simple form of condenser. This stand will focus satisfactorily an oil-immersion objective of $\frac{1}{12}$ focus.

The next step upwards is a model very generally made, with but small variation, under the name of the 'histological' pattern, our typical example in fig. 29, being from Messi's. Watson. In a case without objectives, but one eye-piece, this will cost $4 l$. 10s., or with really good inch and $\frac{1}{6}$ porvers $6 l$. 5 s. Closely similar instruments, but mostly with direct micrometer screw fine-adjustments, as in the succeeding stands, on either claw or horse-shoe feet, are made by Messr's. Baker, Swift, Johnson, and others ; prices being calculated so closely as only to vary a few shillings, for which there is generally sound reason. This type has in fact become so general, that (almost the only stand of which such can be said) it may often be found of good quality in provincial towns, supplied to the shops by anonymous London wholesale makers. It is a handy, neat, well-looking pattern, and the under-stage fitting is of standard $1 \frac{1}{2}$-inch gauge, which allows of a wide range of condensers being used. The stands following, without their focussing sub-stages, also belong to the same rank of instruments. 
But every one who really means to pursue microscopic study of any sort, is again strongly urged to purchase, if possible, an instrument with a focussing and centering sub-

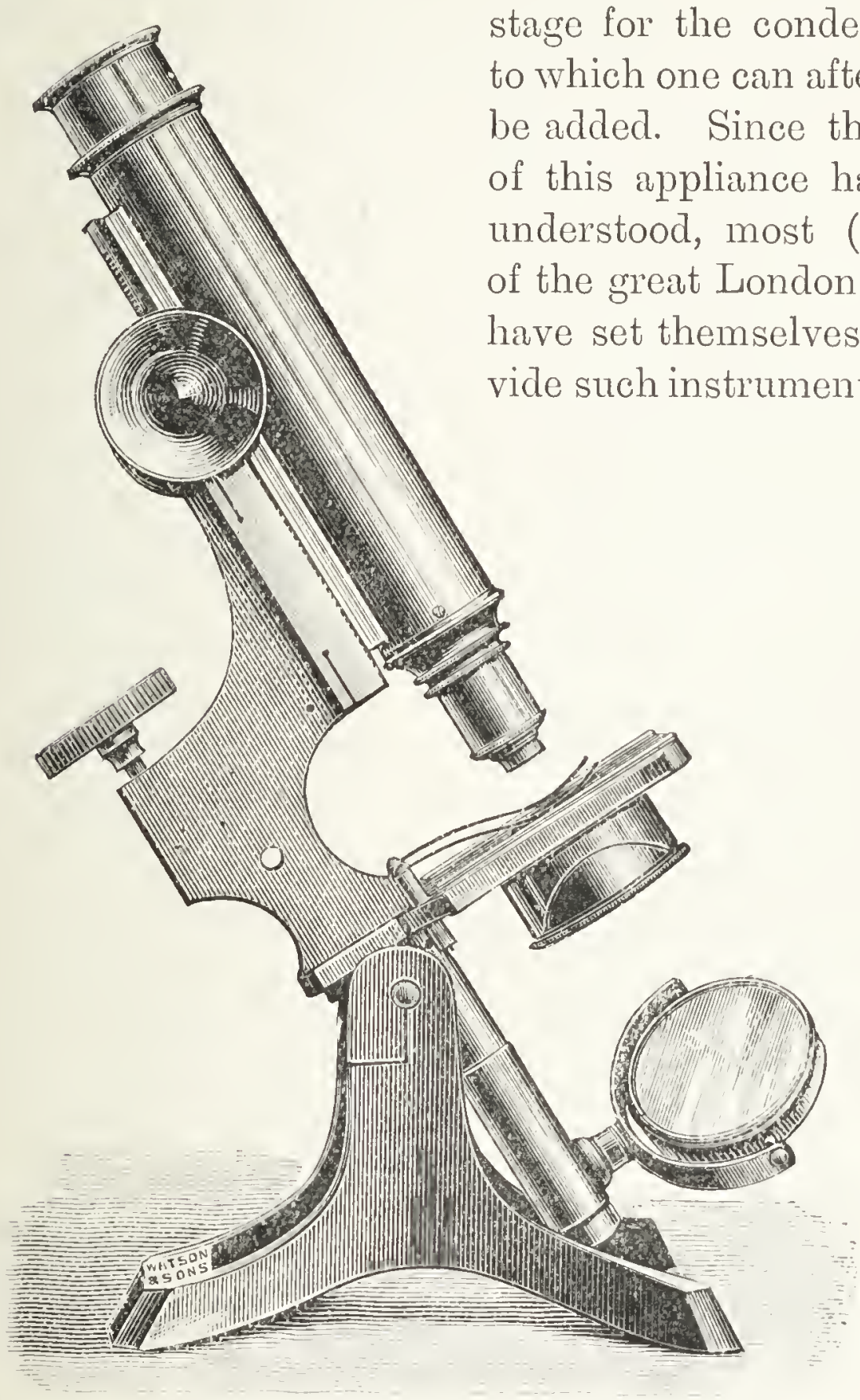

FIG. 29

lowest possible prices for sourd workmanship; and it is worth noticing again how closely the cost of similar work agrees in their hands. Let us see what those who have specially 
studied this class of microscope can give us for our money, especially as it is perhaps in this particular grade that we find the best real value compared with outlay.

Fig. 25, already given on p. 48, represents Messrs. Baker \& Co.'s 'Advanced Student's' pattern, giving a good

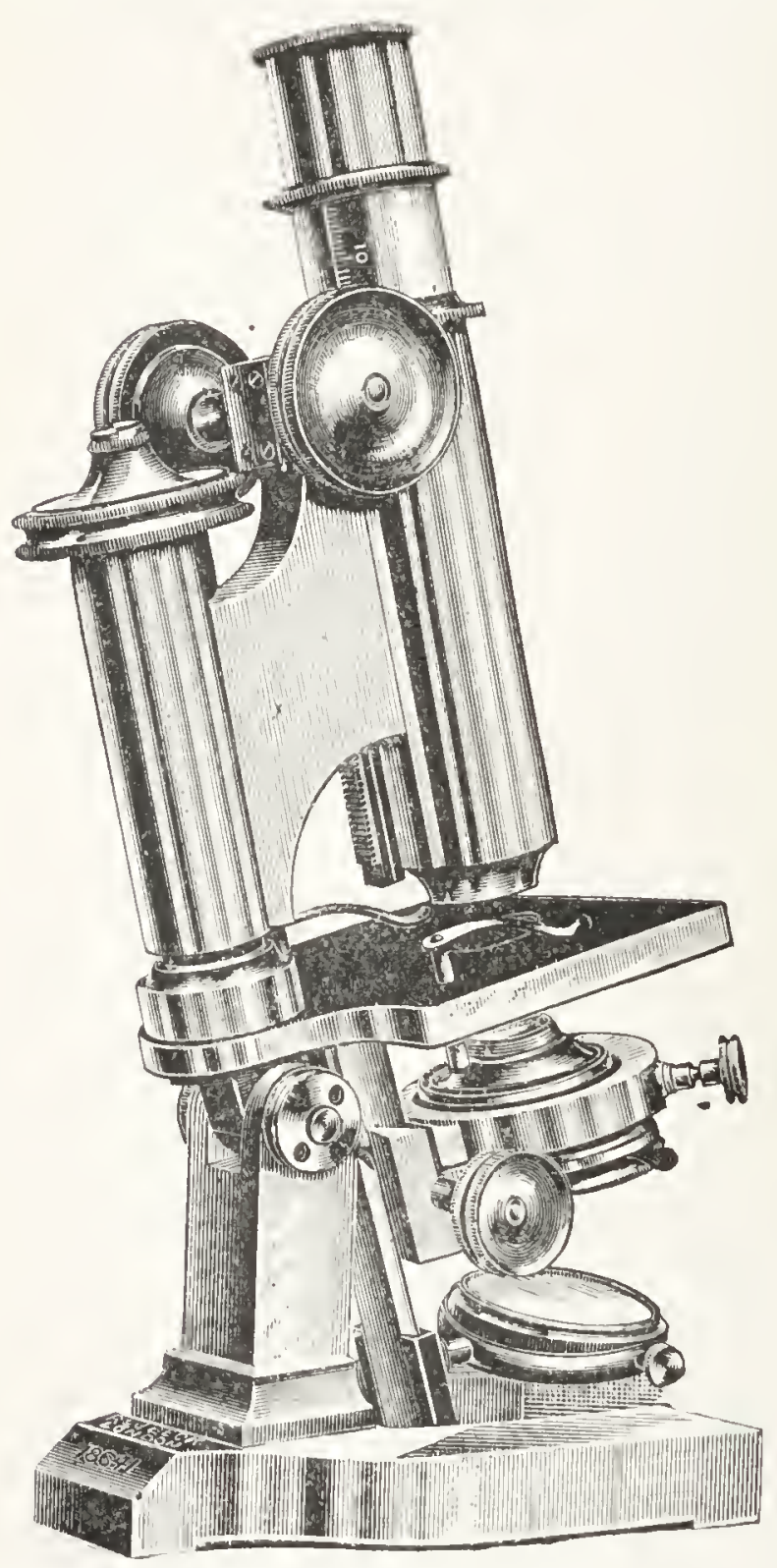

FIG. 30 racked and centered substage, draw-tube, graduated stage with slidingbar, and differential fine adjustment, at the cost of $6 l$. 15s. for the stand alone in case; that is, not including either objectives or eye-pieces.

Fig. 30 shows the same essentials in Beck's 'large model Continental' ' pattern, with a horse-shoe foot. The cost of this stand alone in case is 67.10 s., the stage being plain with spring clips; the sliding-bar will cost a few shillings more. A very similar pattern is obtainable of other makers, and is also made by Leitz of Wetzlar.

Another horse-shoe stand by Newton \& Co. is represented in fig. 31 , on account of the pillar being made reversible, or rather, capable of being turned round. This gives the greatest possible

1 The name is a misnomer, except as regards the foot and fine adjustment. The focussing sub-stage and inclining limb are English details. 
steadiness when the instrument is much inclined, and enables this simple form of stand to be used in a horizontal position for photography. The device was first employed by Nachet many years ago; but I have not seen it again till lately. This stand is sold in case for $6 l .5 \mathrm{~s}$. with single-screw micrometer fine adjustment, or $10 \mathrm{~s}$. more with differential screw.

Curiously enough, just after meeting the above stand it appeared that Messirs. Ross \& Co. had adopted the very same principle of rotating the pillar in a moderate-priced stand-the first time they have catered for this class of purchasers. Their stand is called the 'Eclipse,' and the foot is a turned circular ring, the pillar rising out of one side. It is a firm solid stand, with excellently-protected micrometer fine-motion. They do not quote for the stand alone, but it is sold in case with two eye-pieces, two

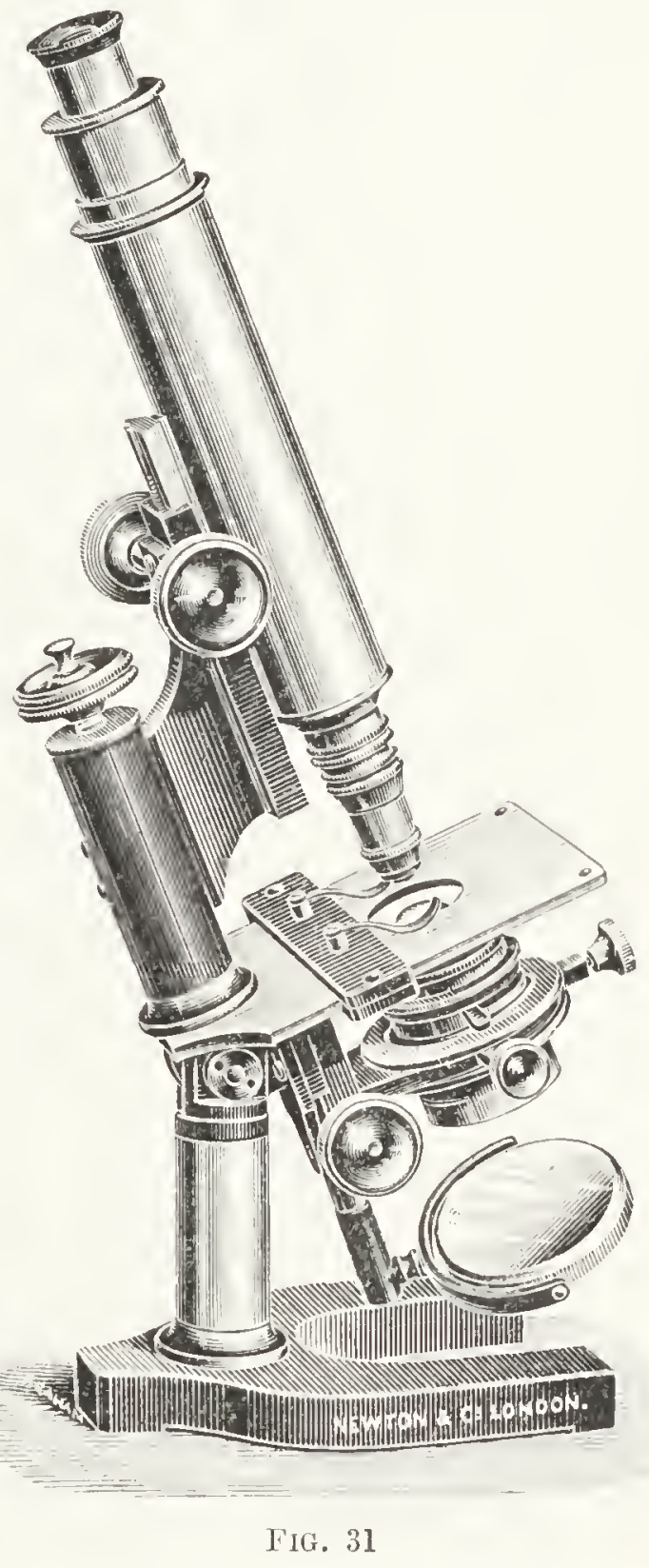
objectives ( 1 inch and $\frac{1}{4}$, or $\frac{2}{3}$ and $\frac{1}{6}$ ), and double nose-piece with the objectives adjusted to same focal plane, for $9 l .18 \mathrm{~s}$. including the racked substage, or 8l. without.

Still seeking really distinctive types, fig. 32 represents a stand by Messrs. Swift \& Son, which has several peculiar 
features. The most conspicuous is the four-legged form of 'tripod,' for it is really a tripod, the back pair swinging on a pivot compensated for wear, so as to adjust on the table like

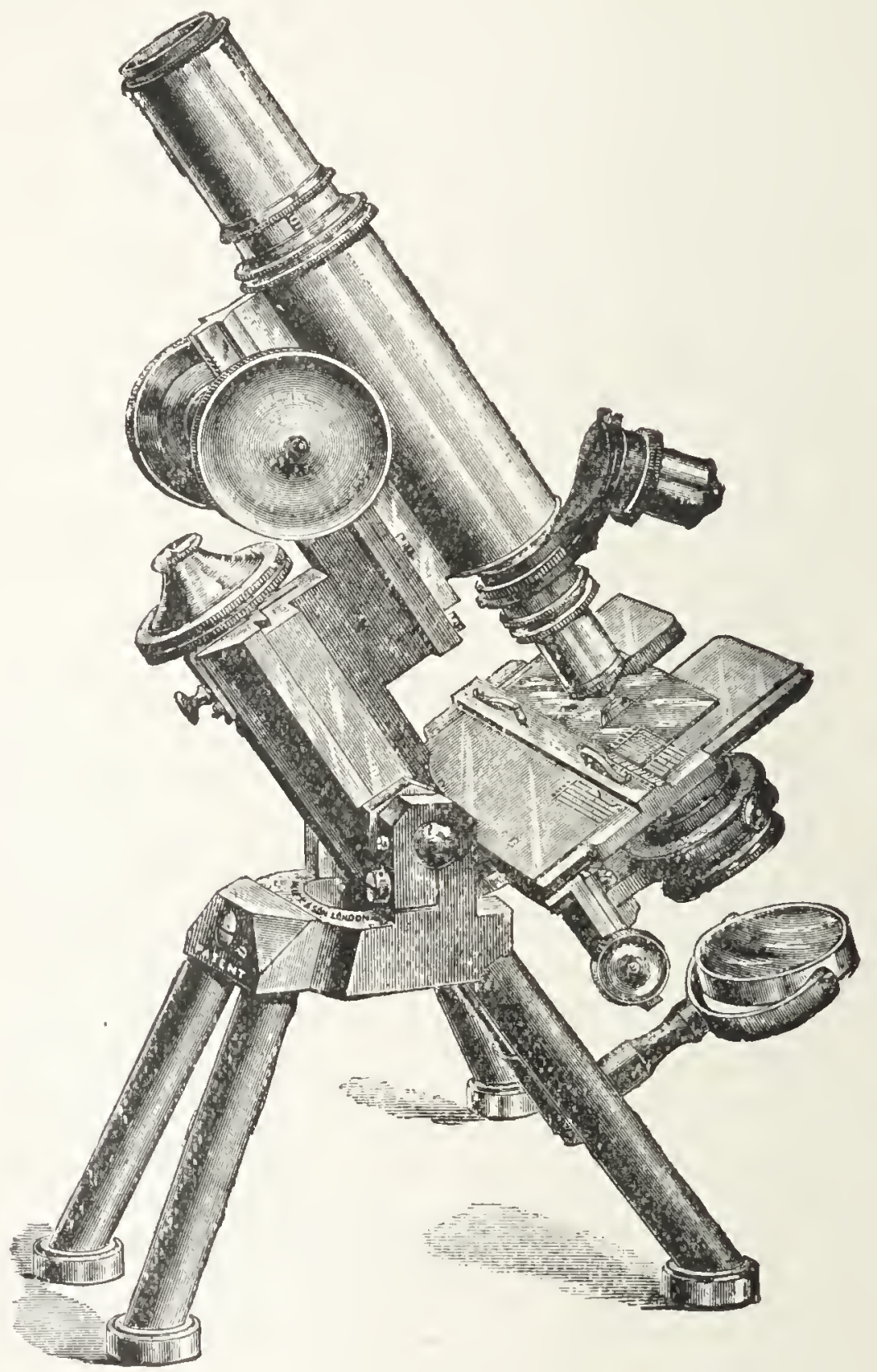

Frix. 32

a single leg. This is therefore an extremely steady stand, yet light and compact; and when the hind legs are swung upwards, the whole actually packs into a box two inches smaller than the three-legged tripod (which is also made, 
exactly similar in other respects). The second feature is, that the micrometer screw works in a half nut, which can be adjusted for wear by the set screw seen at the back under the milled head. A third feature is that a racked and centeling sub-stage is so made, to fixed gauges, that the stand may be bought with only a plain sub-stage ring as in the previous lower class of instruments, while the racked appliance can be purchased later for the sum of 38 s. and be affixed in place by any intelligent person, with no tool except a good small screw-driver. This is a most desirable system; which I am glad to know is gradually extending amongst other cheap microscope stands. The sliding-bar on a divided stage is well seen in this figure, and it will be understood how, by pushing the bar up one division at a time and moving the slide along, every point can be systematically gone over. This stand alone in case will cost 7l. $2 s .6 d$., with three-legged tripod $5 s$. less, or with differential screw 5s. more.

Our last figure of this class of instruments (fig. 33) is given for its distinctive feature of a swinging though racked substage, and is the H pattern of Messr's. Watson's ' Edinburgh' microscope. It is selected also to show a 'mechanical' stage applied to this class of instruments, which costs from 2l. 10 s. to $3 l$. 10s. extra. This particular stage rotates as well. Such an addition makes a really fine instrument of any of this class. The racked and centering sub-stage here swings upon a pivot under the main stage, so as to move quite out of the way when direct light from the mirror is required, and being supported by a catch on the other side when in position. Opinion differs about this method of attachment, which has been condemned; but after repeated examination at intervals of instruments which have been long in constant use, I cas find no solid ground for such a judgment, and prefer such practical tests to forming any $a$ priori opinions. In other microscopes such sub-stages as are 
removable (some are not) slide entirely out in dovetailed grooves. This instrument is sold as here shown, with the mechanical stage, in case without accessories for 10l., with

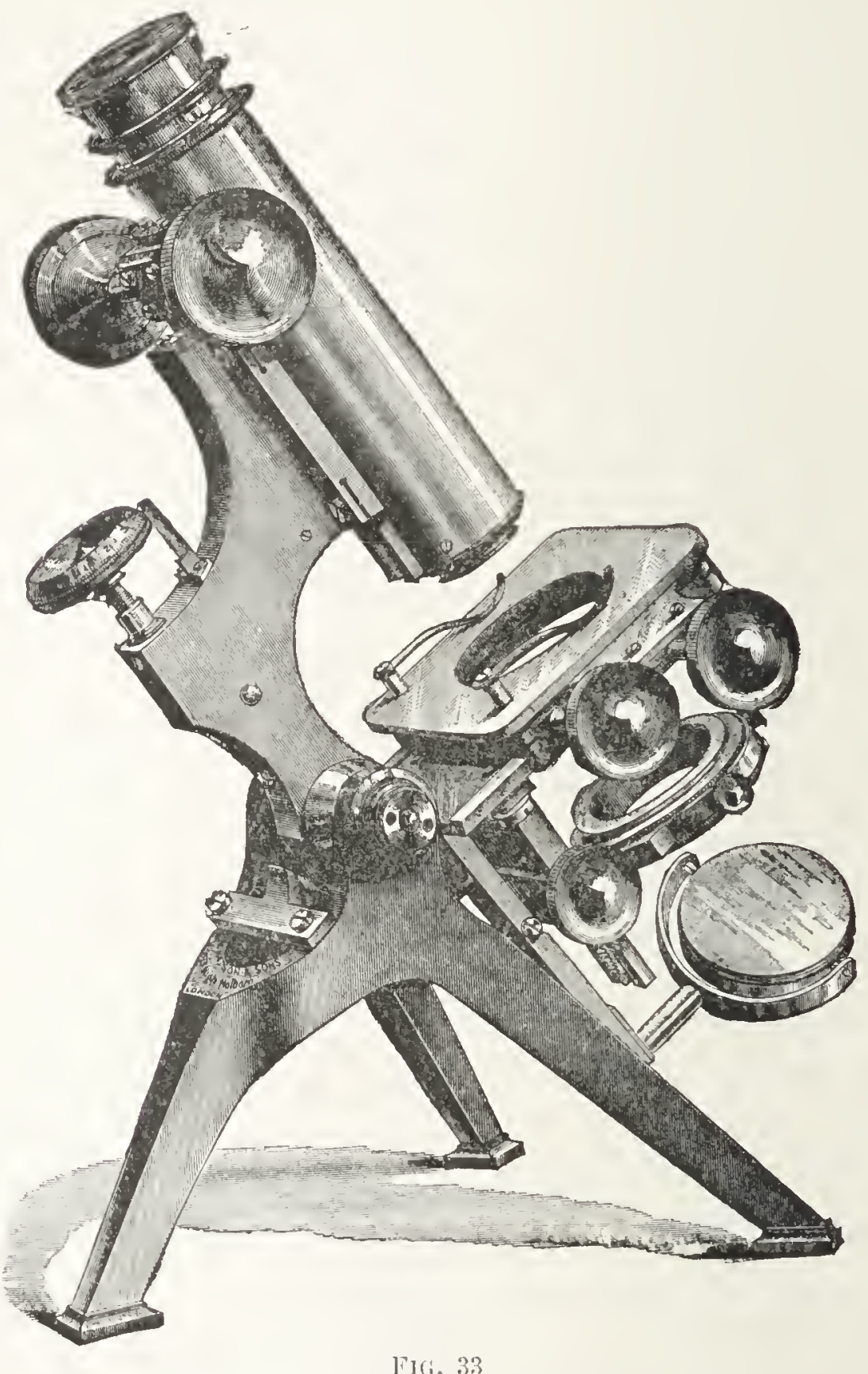

plain stage for 7l., or with hor'se-shoe foot for 6l.6s. For an extra cost of 1l. 5s. an cxtra drau-tube worked by a rack can be added to the body. This extends the range of tube length from about $5 \frac{1}{2}$ inches to 12 inches. It is very 
difficult to get the full range from $6 \frac{1}{2}$ to 10 inches with a single draw-tube.

Any of the plain-stage microscopes of this grade can be had furnished with two eye-pieces, two objectives varying

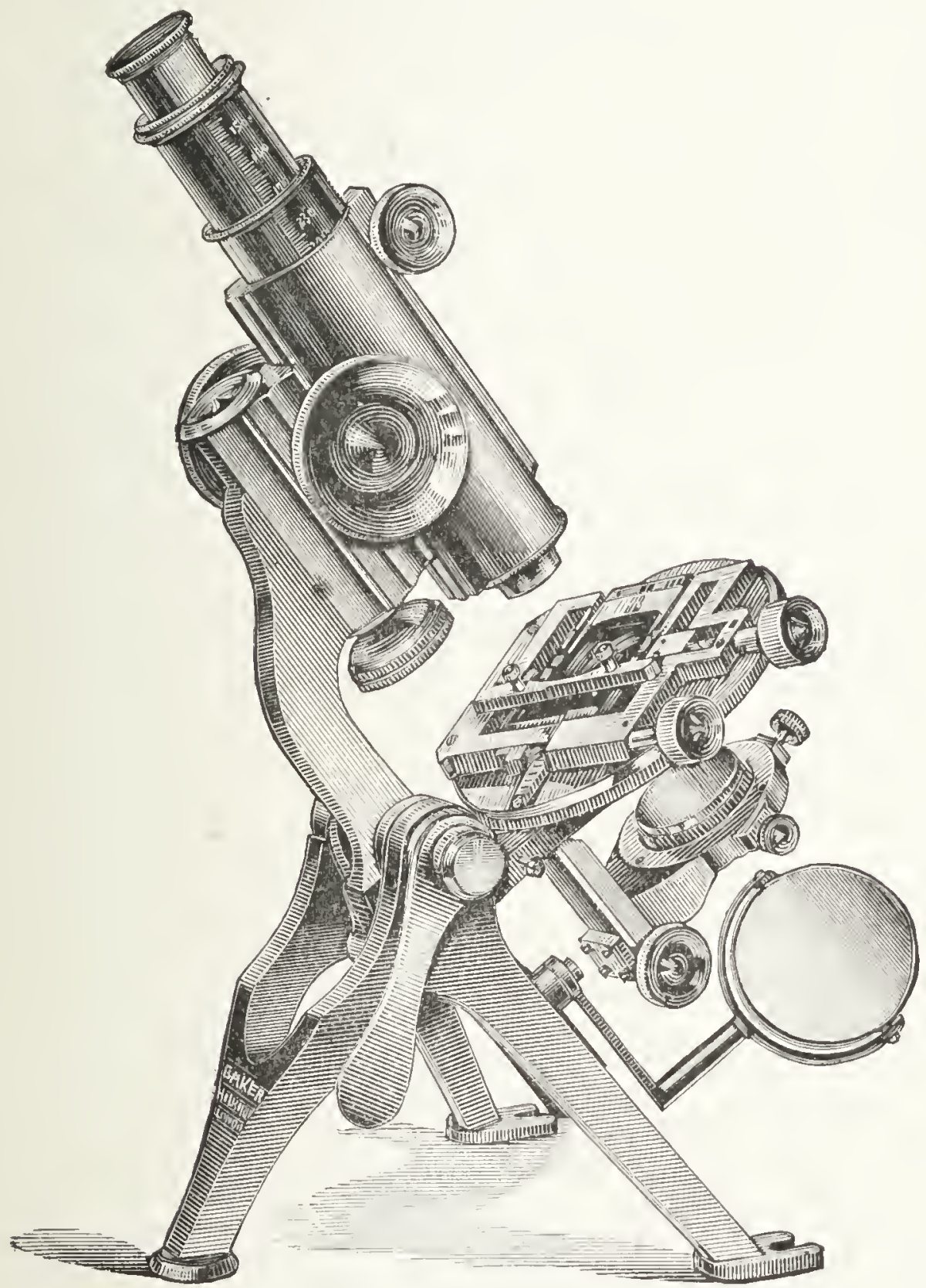

FIG. 34

from 1 inch and $\frac{1}{4}$ inch to $\frac{2}{3}$ inch and $\frac{1}{6}$ inch, and an Abbe sub-condenser with iris diaphragm ( $\$ 39$ ) for an average price of about 11l., varying only a few shillings more or less. With the addition of an oil-immersion objective of high 
power, costing from 5l. to 8l, according to aperture and quality, and a couple of 'compensating' eye-pieces, the microscopist of the present day finds himself in all essential respects better equipped than with any of the older instruments costing 50\%. or 60l, referred to by the Rev. J. G. Wood in a passage already quoted.

Beck's 'Economic' pattern, on a true flat tripod with pillar in the centre, with the addition of the racked sub-stage (this instrument having been originally made without one) also belongs to this class, costing about $1 l$. more, owing to the work in the tripod.

Few readers of these pages will wish for better stands than the foregoing. Any who do so should obtain catalogues of the noble instruments of Messrs. Powell \& Lealand and Messrs. Ross, as well as of the higher types made by the opticians already mentioned, such as Swift's 'Paragon' model, Watson's Van Heurck pattern (an elaborated 'Edinburgh' made to the order of the celebrated Continental microscopist), Beck's 'first-class' stands, or Baker's higher forms of the 'Nelson' model. Let us take one illustration, of the last-mentioned in its smaller form, just to show the directions in which additional refinement is carried in other good instruments as well as that here cited (fig. 34). The whole is carried upon a wide tripod. The mechanical stage is further elaborated, and there is a double draw-tube, one of them divided and with rack-work adjustment, giving lengths from $5 \frac{1}{2}$ to 12 inches; the sub-stage is more substantial and with greater length of focussing adjustment; and there is often added to the sub-stage a fine adjustment by screw motion. The whole instrument is larger and more massive. The cost as figured, in case, is 16l. 16s., which will be two guineas more with fine adjustment to sub-stage (only useful for very critical work with oil-immersion lenses), or four guineas less with a plain stage. Watson's Van Heurck model is of about the same rank in points and price. 
33. Binocular Microscopes.-All the foregoing stands are shown for use with one eye, or are 'monocular' stands. But many microscopes are also made for use with both eyes, or 'binocular.' One reason for this is to obtain somewhat of a stereoscopic effect in examining an object; but a far more important object is to divide the work between the two eyes. Wherever, as in my own case, the eyes are weak or specially sensitive to glare, the relief to both from this is incalculable.

The most frequent binocular arrangement is due to Mr. F. W. Wenham, who made a, free gift of it to the microscopical world. A

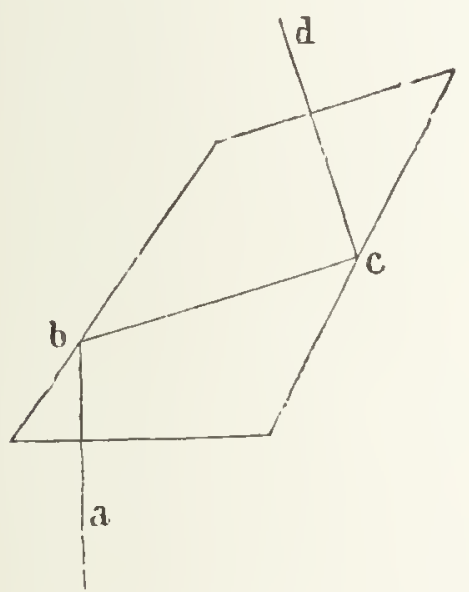

Fig. 35

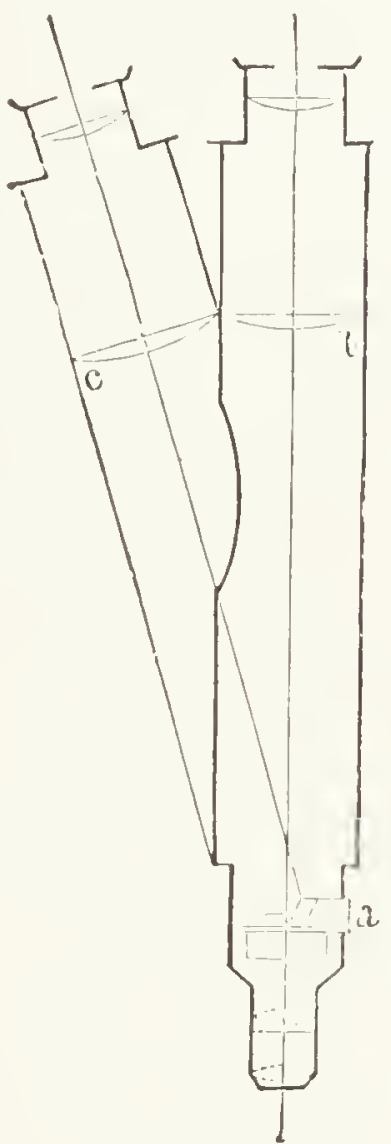

FIG. 36

prism of the form shown in fig. 35 is placed behind the objective, with the lower edge across the centre. This is of such a form that the part of the pencil $a$ which enters the prism is reflected internally in the path $b c d$. The arrangement in the microscope is shown in fig. 36 , one half the pencil proceeding direct up the primary tube $b$, while the other half is directed up the inclined tube $c$. The prism is so mounted at a (fig. 36 ) that it can be drawn out of the way at 
pleasure, when the instrument becomes monocular. This is its great advantage ; the next being cheapness and simplicity, so that a binocular arrangement can be had for an extra cost of $2 l .10 \mathrm{~s}$. to $3 l .10 \mathrm{~s}$. The disadvantages are, however, several. The system is not well adapted for objectives higher in power than about $\frac{4}{10}$, and of angles above $50^{\circ}$ in air, though higher powers can be used by having them specially mounted in shorter cells, so that the back lens comes close to the Wenham prism. With high angles, however, the stereoscopic effect becomes painfully exaggerated. The most serious defect is that a long, extending draw-tube becomes impossible, as the tubes must be cut away on their inner sides. Some draw-adjustment is necessary, in order to provide for the varying distance between people's eyes; but it can hardly exceed at the most $1 \frac{1}{2}$ inch. Hence a Wenham birocular is difficult to use with objectives of both Continental and English tube-lengths. The best compromise is to have a length that will rack down to 7 inches, which most short-tube objectives will bear fairly well; this will rack out to $8 \frac{1}{2}$ inches, and then a 'lengthening adapter' can be inserted in the monocular body for use on critical work with long-tube objectives.

Both Messrs. Ross, Watson, and Powell \& Lealand supply other prism arrangements which will work up to $\frac{1}{15}$ immersions; but all of them are necessarily much more expensive that the Wenham prism. Some are non-stereoscopic, others stereoscopic. The average cost varies from 5l. to $8 l$, , or more. The non-stereoscopic is, I think, to be preferred, the relief to the eyes being the main point, and well worth the cost whenever a long microscopic career is in view and the eyes are specially sensitive to glare.

There are also binocular eye-pieces constructed, which fit into a single body-tube. The best is probably Abbe's, made by Zeiss of Jena, and costs about $8 l$. This requires a tube not more than $170 \mathrm{~mm}$., and is therefore preferable for short- 
length microscopes. Its weak point is that the eye-pieces have to be specially constructed for it; an ordinary pair cannot be used.

34. Objectives.-The advance in these is even greater than in stands, both as regards the highest class and those sold at a cheap rate. A few hints may assist the reader to get value for his money.

Except under first-rate advice, none should be purchased but such as bear the name of a maker with a present reputation. Many lenses, good for their day, may be offered for a third of their cost, and yet be dear now.

Most of such stands as have been described are habitually sold with pairs of objectives, usually either 1 inch and $\frac{1}{4}$ inch, or $\frac{2}{3}$ and $\frac{1}{6}$. Often both pairs are made, the first being preferred by ordinary users, and the higher powers by medical students. Such pairs of objectives being required in numbers, special pains have been bestowed in working them out; and almost without exception they will be found sound and good lenses, of a defining power unobtainable at twice the cost years ago. To buy a stand thus equipped usually saves about 10 s. on the total, and the lenses will never cease to be useful, whatever may be acquired later on. The beginner should, however, also have a lower power, say 2 inches or thereabouts. These can be had cheap, of German make; English powers may cost from $25 \mathrm{~s}$. upwards, but are much better.

The German makers-Zeiss, Reichert, Leitz, and Seibert -also make really fine but cheap lenses of the usual powers. Every maker, without exception, is found to have certain powers in his series better than the rest, which are soon recognised by workers and become favourites. Of Seibert's I have found a dry $\frac{4}{10}$ at 21 s. and a water immersion $\frac{1}{16}$ at $3 l$. the best. Reichert's No. $3\left(\frac{2}{3}\right)$ at 21 s., No. 6 $\left(\frac{1}{5}\right)$ of 0.77 N. A. at $30 \mathrm{~s}$, and No. 7 A $\left(\frac{1}{7}\right)$ of N. A. 0.82 at 36s. are very fine. The same maker supplies a good oil 
12 for $5 l$., but the best of his ordinary series is an oilimmersion of $\frac{1}{7}$ real focus, and $1.30 \mathrm{~N}$. A., sold for $8 l$., which no eye-piecing has broken down. Twenty years ago, no lens then made, at any price, would have borne such a test. A new oil $]_{1}^{1} \frac{1}{2}$ of same aperture and price is another excellent objective. A semi-apochromatic of $8 \mathrm{~mm}$. focus and $0.50 \mathrm{~N}$. A., sold for $32 s$., is also an admirable and well-known lens.

Leitz makes a 'pantochromatic' (lettered $\mathrm{P}$ ) as well as achromatic series. His ordinary No. $2\left(1 \frac{1}{4}\right)$ and No. $3\left(\frac{2}{3}\right)$ at 15s. each are as good of their power as it is easy to find; No. $7\left(\frac{1}{6}\right)$ at $30 \mathrm{~s}$. is also good, and the oil-immersion $\frac{1}{12}$ at $5 l$. is an excellent lens. The pantachromatics, though more costly, I did not find so good for general work, with two exceptions. ${ }^{1}$ These are the $\mathrm{P} 3$, a dry $\frac{1}{8}$ with correction-collar' 0.87 N. A., sold at $3 l$, and an oil $\frac{1}{12}$ of 1.30 at $8 l$. The former was one of the best dry $\frac{1}{8}$ objectives I ever met with.

Zeiss's series are rather more costly than the above. The best of them, I think, are the $a$ a, at $27 \mathrm{~s}$., which is a very fine inch power, and the D ( $\frac{1}{6}$ of $0.65 \mathrm{~N}$. A.) at $2 l .2 s$. Their quality is very uniform, and definition admirable, but they are not so flat in field as the above.

Of English lenses, the 'pairs' most in demand have already been named; all such powers are excellent, from any of the recognised makers. Watson's dry $\frac{1}{6}$, at $2 l$., is a wellknown lens. So also is Swifts' dry $\frac{1}{6}$, at $36 s$., and two oil $\frac{1}{12}$ at $5 l$. and $7 l$ : of their general series the most noteworthy are the 2 -inch, $33 s ., \frac{1}{2}$ at $2 l .12 s ., \frac{1}{3}$ at $32 s$. , and a $\frac{1}{5}$ of N. A. 0.87 at $3 l .{ }^{2}$ The cheapest really good ?-inch made, up to the date this is written, is probably Beck's, of $\mathrm{S0}^{\circ}$ air angle, at $25 \mathrm{~s}$. One of these (taken at random) bore a $\times 12$ eye-piece well, and $\mathrm{a} \times 18$ one fairly, on the usual tests. Their $\frac{4}{10}$ of 'air'

1 The series examined was the first sent to this country.

2 After above was in type I received from them a new $\frac{2}{3}$ of N. A. 0.30 and $\frac{1}{2}$ of 0.50 , at 30 s. and 40 s., both flat in field, and on most objects bearing $a \times 27$ ocular. The $\frac{1}{2}$-inch is the best of that power, for general work, which I have yet met with. 
angle $95^{\circ}$, with a correction collar, at $5 l$., is the cheapest objective of such wide aperture obtainable, and a really magnificent lens, which it is not so very easy to distinguish in performance from an apochromatic. As a rule, however, Messrs. Beck devote attention to smaller apertures, for histological students, and while this is written are preparing a low-aperture oil $\frac{1}{1}$ to be sold for $4 l$.

Messrs. Ross \& Co. have very recently indeed brought out a new set of lenses from Dr. Schroder's calculations. An inch of $35^{\circ}$ at $3 l$. $3 s$. is very fine; but the most noticeable value was in an oil $\frac{1}{8}$ of 1.20 at $7 l$., and an oil $\frac{1}{12}$ of 1.25 at $7 l$. 10s. The $\frac{1}{8}$ proved to have a little more aperture than was stated, and was if anything the best, bearing the highest ocular well on Podura with unusually little residual colour. Both were however very fine powers for their apertures, and flatter than usual in field.

35. Apochromatic 0bjectives.-These are so high in price that probably few readers will procure them, and a few words must suffice, as they are only needed for the highest class of serious work. In flatness of field the Zeiss series are (or have been up to the date this is written) defective, but in definition and in uniformity of quality they excel most of those made by other opticians. The $\frac{1}{2}$-inch of N. A. 65 , and the oil-immersion $\frac{1}{8}$ of N. A. 1·40, are probably the finest objectives yet produced, as regards definition over a small field; and as their power to bear high eye-piecing enables the $\frac{1}{2}$-inch to give as much magnification as the $\frac{1}{8}$-inch with a lower power, two such lenses alone will cover an immense range of work. The present prices of these two powers (reduced 20 per cent. since the early lists) are $7 l$. for the $\frac{1}{2}$-inch and $20 l$. for the $\frac{1}{8}$ immersion, others being in proportion. The cheapest high-power apochromatics are the $\frac{1}{12}$ oil-immersions by Powell \& Lealand, at $10 l$, most of which are of high quality, though not I think strictly free from residual colour. Powell \& Lealand's I 
have found generally flatter in field than those by Zeiss, but not quite so uniform: the best of them are certainly amongst the finest in the world.

36. Qualities of Objectives.-The various points desirable in an object-glass have not the same relative value for all workers; and some being more or less inconsistent with others, a few words may be of service respecting them.

Definition, or that quality by which a fine line is represented in the image by a fine sharp line, and not a blurred or ' woolly' line, is, however, in all cases the first property necessary. Nothing whatever can compensate for the want of this, under the eye-piece power required. In the extent of the eye-piece power it will bear, lenses vary. Thus a cheap lens, which remains sharp under an A or B eye-piece, may suffice if the work does not require more. Higher powers especially, however, should bear high-power eye-pieces.

Apcrturc. - The bearing of angular aperture upon resolution, or separation of minute detail, has been referred to in \$22. For a certain number of markings per inch, the lens must have a certain aperture. Hence magnification without corresponding aperture is of no account; and, on the other hand, it is important to know that, if we have the aperture, a lower power is as good as a higher one, provided it will bear eye-piecing up to the needed magnification. No one now uses $\frac{1}{25}$ and $\frac{1}{50}$ powers. But the value of great aperture differs with the work. The student of diatom-marking, or of bacteriology, must have it; for ordinary current use moderate apertures will suffice, and will suit better, as giving more working distance, and entailing less cost, and giving more of what is called

Penctration, or depth of focus. - This power to see something in more than the exact focal plane, decreases with the aperture. Hence high apertures are disliked by biological students. It is true that an apochromatic objective of lower power will have still more penetration though of high 
aperture, while it can be brought up in power by eye-piecing. Better results can therefore be got in that way, if cost be no object. But the student can rarely afford such high-class lenses, and hence he prefers moderate aperture.

Working distance.-Objectives being compound, the optical centre from which focus measures is within the system, and the clear space between the front surface and the coverglass, called 'working distance,' is much less than the focal length. This also decreases with aperture. To a great extent the same remarks apply as in the last paragraph, and sufficient but moderate apertures with good working distance, will best suit general purposes and most present readers.

Flatness of field means that the image of a really flat object should be in focus over the entire field of the eyepiece. The necessity for this quality differ's with the work and the objectives and oculars used. Formerly, with low eye-pieces, it was of very great importance; and for miscellaneous work it is still so, especially in examining small forms of life, such as rotifers or infusoria. To work on these with comfort, they must be comfortably in focus over the large field of a low eye-piece. Unfortunately, the tendency to use apochromatic and other high-class lenses with high-power compensating eye-pieces which have a small field, has led to great and careless disregard of this useful quality. Hence some of the most costly lenses are really unfit for certain classes of work, for which, if only flat in the field, they would be perfectly suitable.

To be practical, an inch power should, if it can be afforded, be about $25^{\circ}$ air angle ; a $\frac{1}{2}$-inch, not less than $40^{\circ}$, or $\frac{4}{10}$ than $50^{\circ}$; a $\frac{1}{4}$ or $\frac{1}{5}$ from $90^{\circ}$ to $100^{\circ}$; a $\frac{1}{6}$ from $110^{\circ}$ to $120^{\circ}$, for general purposes. These angles allow of good working distance. For bacteria, higher apertures are better if they are within the means of the purchaser; for systematic study in cell life, such as Dr. Dallinger occupies himself with, they are necessary. 
37. Testing Objectives.-The novice cannot possibly test objectives; and the highest class can only be exhaustively tested by methods which require long experience to master. Should however even a novice purchase higher-priced objectives than the 'pair's' usually sold with a first micro-

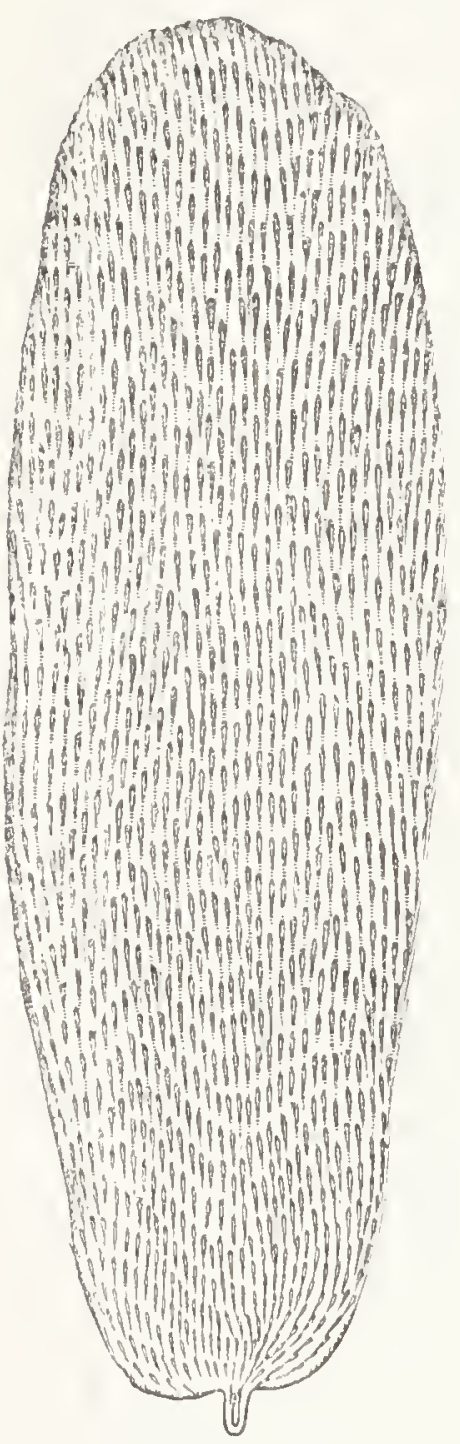

FIG. 37.- Test Podura Scale scope, he may reasonably ask to be shown certain things; and the few hints here given must be confined to these, and to such tests as can be grasped with moderate experience, and yet will prove a lens to be of very fairly good quality. They must be read in connection with $\$ \$ 53$ and 60 .

Good powers from inch to half-inch or $\frac{4}{10}$, should stand a two-thirds to threequarter cone easily ( $\$ 53$ ), and with this can be very fairly tested by the numerous small hairs which cover the whole surface of the tip of a blowfly's proboscis. With such cones, all these which are in focus should be shown as sharp as needle-points, with no nebulousness or fringes.

For $\frac{1}{3}, \frac{1}{4}, \frac{1}{5}$, and even immersions, there is no better test of definition than a 'test' Podura-scale, known as Lcpidocyrtis curvicollis. ${ }^{1}$ Fig. 37 is a reproduction by photography of the late Mr. R. Beck's original drawing (which has never been equalled) of this scale, as seen under a $\frac{1}{4}$-inch. With even a good lens it should be observed with

1 A slide of the 'test, coarse Podura' should be asked for. This and the other tests named can usually be obtained of Messrs. Watson or Balier: the scales often of Messrs. Beck. All microscope houses do not keep them. Test diatoms should be by Müller or Thum of Germany, either 'dry,' or' mounted in dense media, which shows up their details more strongly. 
a 'cone' of only half the objective aperture (\$53). Then, if the lens is well corrected, the scale will be covered with distinct 'exclamation marks' as in the figure, each black mark with a light streak in the centre. The width of the white streak will differ with the precise focus, and the markings may vary a little. The essential point is the sharpness of this black and white marking; and the test of the lens is the eye-piecing which it will stand without breaking down. A first.class dry lens will stand the Zeiss $\times 27$ compensating ocular; but it is a very fair glass that will bear $\times 18$.

We also need tests, however, which embrace more of the aperture. For a $\frac{1}{4}, \frac{1}{5}$, or $\frac{1}{6}$, the Pleurosigma angulatum mounted dry is still a good test, though the old so-called 'resolution' is worthless. But if with a full three-quarter' cone $(\$ 53)$ a lens will give, with the $\times 27$ ocular, either sharp white discs, or clean black dots, with precision, according to the focus, the lens is not a bad one. A firstclass $\frac{1}{6}$ ought to do a little more, and show at least indications of the $N$. rhomboides next mentioned.

Ground-glass illumination ( $\$ 54$ ) will much diminish a beginner's difficulty in illuminating some of the tests here mentioned.

Immersions must be tested more highly; but the mere 'resolution ' of $A$. pellucida, once so much talked of, is worthless. It is a mere test of aperture, and of the operator, and of the slide; in fact A. pellucida may be 'resolved' by a really bad lens. The following two diatoms interpose no great difficulty, and yet the lens cannot be a bad one that succeeds. They are Navicula rhomboides and Amphipleura Lindheimeri, and should be procured mounted in styrax or equivalent media: an extra one of each, finely ground on the back, will also be useful. With a large central cone, the dots round the ends of the Navicula should appear distinctly black or white, with a high-power ocular. (I never found 
these dots sharp, however, like others: there is always some woolliness about them.) And the Amphipleura should also be capable of being dotted black or white, at least on a portion of any valve preserving the outer membrane, where the marking is always bolder; but even the finer marking ought to be clearly dotted. The last is the severer test, and will need care; but both interpose no real difficulty with a really fine lens, and for that very reason are selected. But though they put no great demand upon the operator, the objective which will pass them, with Zeiss's highest-power compensating ocular, is a very good one indeed. Care must be taken to choose a valve which lies flat on the slide.

Slides of some bacteria-of leprosy, or Koch's 'comma' cholera bacillus, or tubercle-are also exceedingly good tests. They should be examined with a large cone-from two-thirds to three-quarters - and should then appear sharp and thin. But they will almost never bear such high-power oculars as the preceding: the fact is their exceeding fineness severely tests the correction of the ocular as well as of the objectives, and all compensating oculars are by no means alike good.

38. Eye-pieces.-The construction of the Huygenian eyepiece was described on p. 21. This is the most usual form, and costs from 7 s. 6 d. each for the small size used on smaller stands, to $1 l$. or more for those with caps, for larger tubes. There is an accepted gauge for the small ones; unfortunately there is none such for the larger, which is to be regretted. The ocular lens, instead of being simple, is sometimes made achromatic, which gives better definition with the higher powers.

Compensating Eyc-picces have correction carried to an excess in the ocular lens, to counteract certain errors it is almost impossible to correct in high-power objectives, or others made with single hemispherical fronts. The performance of all such lenses, whether 'apochromatic' or not, 
is greatly improved by this compensating error introduced into the eye-piece; but ordinary lower power's are deteriorated. To allow the same eye-pieces to be used throughout, the Abbe-Zeiss apochromatics have therefore similar error's artificially introduced into the lower powers as well; and it is to be hoped that this example may be followed in future high-class objectives. The lower power compensating eyepieces are Huygenian; the higher are combinations with no field lens, working as a single or positive eye-piece.

The Kelner Eye-piece has an achromatic meniscus for the ocular lens, and a double-convex field lens in its focus. It gives a very large and flat field, but of bad definition; and the least speck on the field lens is, of course, in focus, and unpleasantly conspicuous.

Ramsden's eye-piece consists of two plano-convex lenses with their convex surfaces towards each other, at such a distance that the focal plane is a little outside the field lens; hence this eye-piece is 'positive.' It is chiefly used for micrometry, but it is more usual now to insert a micrometer in the focal plane of a Huygenian eye-piece.

English oculars are generally called either A, B, c, D, E, or No. 1, 2, 3, 4, 5, and the magnification of either is not uniform. Zeiss professes to remedy this indefiniteness in his 'compensating' series, by marking each with its actual magnifying power: e.g. 18 or 27 is supposed to mean that the eye-piece magnifies the original image eighteen or twenty-seven times. Some other maker's follow this plan in their. 'compensating' eye-pieces, and it is to be hoped it will spread to all. Unfortunately, however, Zeiss does not carry out the plan consistently, but only for the long or 10-inch tube. For this the stated magnifications are correct. But the eye-piece marked 18 for the 6-inch tube is the same power as the 27 for the 10-inch tube ; being in this case marked 18 on the fictitious supposition that the image is the same size with the short tube. Thus, a $\frac{1}{4}$-inch gives 
an original image $\times 40$ at 10 inches, and $\mathrm{a} \times 12$ eye-piece gives $\times 480$. With the short tube the same lens and eyepiece give only $\times 320$. By the absurd fiction referred to (doubly absurd when 'accuracy' is specially claimed) this is reckoned as made up of objective still $\times 40$ and eye-piece now marked $\times 8$; whereas in fact the figures are, objective about $\times 26$, eye-piece as before $\times 12$. It is to be wished that this foolish pretence should be done away.

Flatness of field partly depends upon the eye-piece, as intimated on p. 22. It can be increased in the Huygenian forms, by bringing the field lens slightly nearer the field diaphragm, and also by substituting for the plano-convex a slightly meniscus field lens. With achromatic or 'compensated' ocular lenses, there need be no loss of definition from this, and my own best compensating 'projection' eye-pieces are specially constructed upon this plan.

39. Illuminating Apparatus.-The mode of illumination will vary, as the object or slide examined is transparent or opaque. The vast majority are transparent, or examined by light sent through them from the under side. For a large number of ordinary subjects the concave side of the mirror is sufficient; but for high powers or critical work this is not sufficient. Formerly a large number of appliances were in use, called condenser, spot-lens, paraboloid, and many 'illuminators.' The most valuable modern improvement and simplification of the microscope is to make an improved 'condenser' alone, properly mounted, answer' all purposes.

Fig. 38 is a section of the condenser in most general use amongst medical students, known as the Abbe model, and which when racked up to the under side of the slide, with a large drop of cedar oil ${ }^{1}$ between (else rays above N. A. 1·0 could not emerge, as explained in $\$ 6$ ), will give rays of N. A. 1.40 at the margin of the cone of light. The chromatic and spherical aberrations are however enormous, the focus

1 A slip of cover-glass is also used, to fill up the interval. 
of the marginal rays being much shorter than of the central part of the cone; hence the solid cone of focussed light, which alone can be used in critical work, does not exceed N. A. 0.5. For the simple under-stage tube shown in fig. 29 this condenser is mounted as in fig. 39, to push in from beneath, with an iris diaphragm underneath which contracts, on moving a projecting arm, to an aperture as small as desired, and a flanged circle which swings outwards, to carry various central stops for manipulating the light. The condenser mounted either thus, or to fit from above into the substage as in fig. 40, will cost about $2 l .2 s$. A cheaper form of two lenses, of N.A.1.2, will be about $7 \mathrm{~s}$. 6d. less. The best condenser of this form is Powell's, made on the same

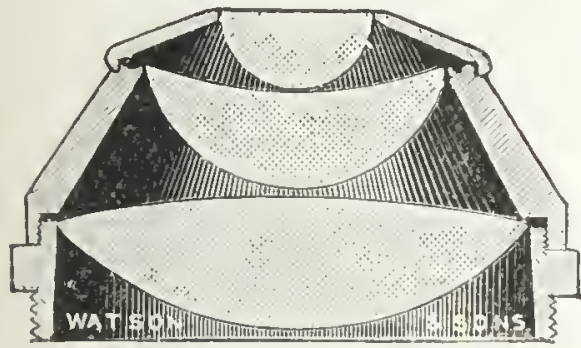

FlG. 38

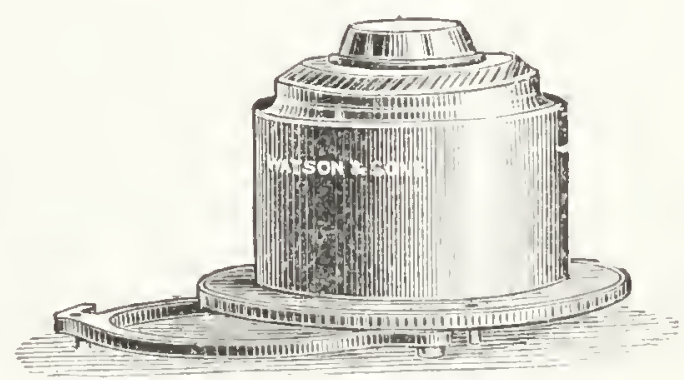

F」G. 39

system but of much smaller diameter and focus; the optical part alone of this costs $2 l$., and has to be mounted over the iris and stop mount, which will cost about 25 s. besides.

A much better condenser is the achromatic Abbe form, shown in fig. 40 mounted over the iris diaphragm and stopcarrier, and so as to fit from above into substage, but it can also be had reversed as in fig. 39. This gives an N. A. of $1 \cdot 0$ only; that is, the largest cone of light which can emerge from ' dry' lenses; but the 'solid' cone available for critical work (see $\$ 53$ ) is 0.65 as made in Germany. Some English ones are rather better, and I met with one giving nearly N. A. $0 \cdot 70$ of solid cone. The cost, with mount and a set of stops complete, will be about 3l.15s. This condenser does 
excellent work all round, and also in photography, and is probably the most generally useful.

Condensers giving far higher aplanatic cones of light are made by the great English opticians, but are only needed for high-class work, with the best objectives. Powell's dry apochromatic gives nearly 0.9 of aplanatic cone, but the optical part alone costs $6 l$., while his dry achromatic (mounted) is $7 l$. Messis. Swift make an excellent dry apochromatic for $4 l .10 \mathrm{~s}$, without the iris, and an achromatic at 3l. This latter, with

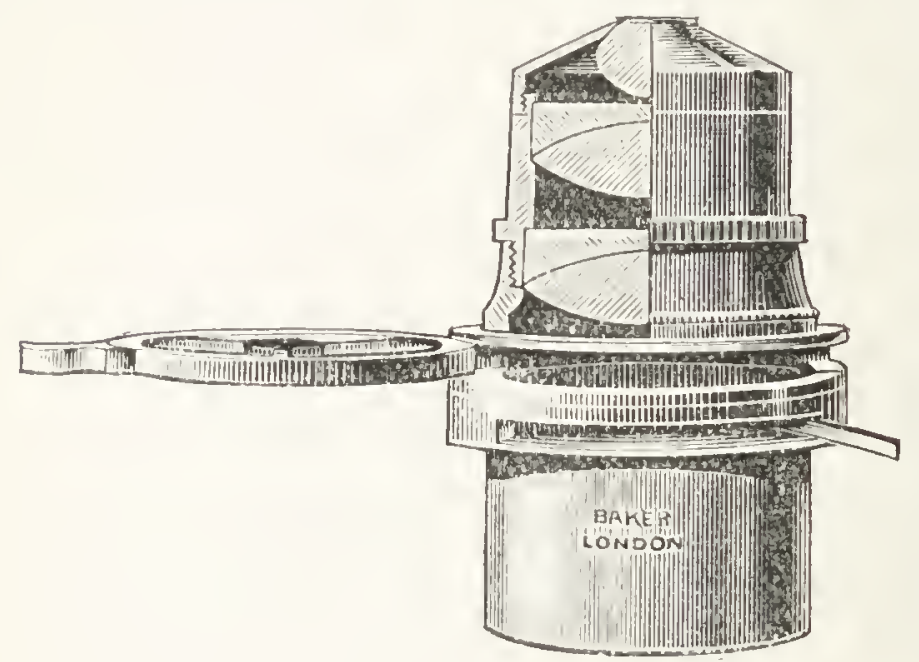

FIG. 40 iris and stop mount, will cost about the same as the Abbe achromatic, but gives a far larger aplanatic cone for use with high powers. Messis. Beck also make a good achromatic condenser of about the same figures; and Messrs. Watson are working out an apochromatic whilst these pages are in the press.

No expensive condenser will be needed at first to utilise the substage. Any lens of suitable size and focus, mounted in a plain wooden ring with a flange, which can be dropped into the substage, and focussed on the object so that the image of the flame is focussed by the objective together with the object, will act as a condenser, and on all but coarse objects improve the image. One of the achromatic Steinheil lenses shown in fig. 21, thus mounted and used, will make an excellent condenser of moderate angle, sufficient for all early work, up to a $\frac{1}{4}$-inch objective. Various condensers of moderate angles have long been sold under various forms, as Webster's, Gillett's, \&c. If such a one is attainable at a low 
price, it is not to be despised; but for purchase as new apparatus, those above named are to be preferred.

The forms known as a 'spot-lens' and 'paraboloid,' for' exhibiting transparent objects as if self-luminous on a dark field of view, are referred to in the next chapter. A really good condenser, however, does for all.

Opaque objects have to be illuminated quite differently; the necessary appliances will also be best dealt with when treating of their management.

40. Polarising Apparatus.-It is impossible here to explain in detail the nature and effects of polarised light. It must suffice to say that the vibrations in light-waves are across

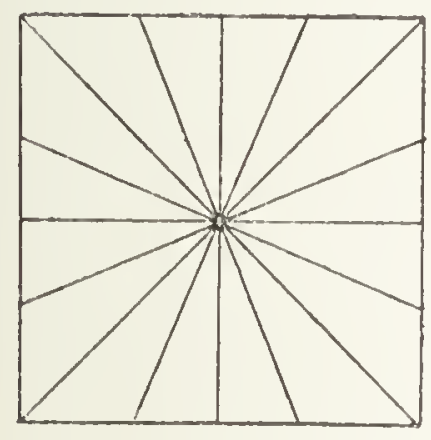

FIG. 41

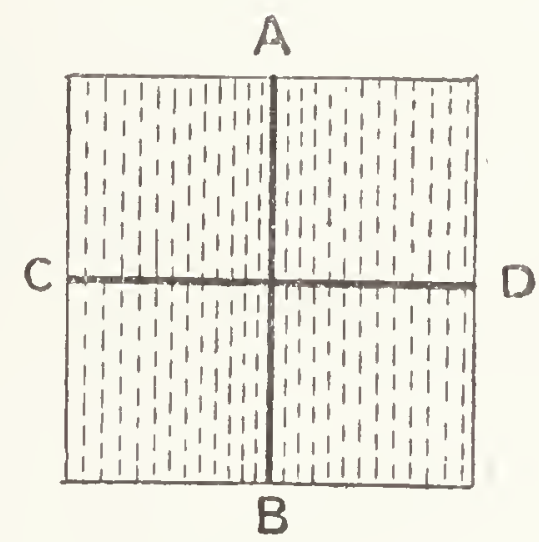

FiG. 42

the line of the ray, as when a stretched cord is vibrated. In common light this vibration is in all planes across the ray, so that in a beam of light coming through a pane of homogeneous substance, like glass, the vibrations may be considered as in fig. 41. But if the substance be considered to have a decided grain, as in fig. 42, like a deal board, or to be unequally elastic in different directions, like many crystals (not all), then these vibrations are all 'resolved' into the two direetions at right angles, A B, CD, (fig. 42), which are the directions of greatest and least elasticity. In a single thin film of the crystal we can see 
nothing of this, but it is so ; ${ }^{1}$ and as the two parts of the beam of light, now termed plane-polarised (for the vibrations can be by more complicated arrangements also polarised in a circular or elliptical path), are differently refracted, or the same crystal has different indices of refraction ( $\$$ ) for the two polarised rays, consequently the two parts are 'totally reflected' at different angles. Hence, by cutting a piece of Iceland spar in two at a particular angle and joining together again, one of the two polarised pencils can be totally reflected to one side, while the other is transmitted, and we now get, through the 'Nicol prism' as it is called, only half the beam of light, polarised only in one plane. Still we see no difference except loss of half the brightness; but if, holding one Nicol prism against the sky, we look at it through a second which we turn round, we find when the two are 'crossed' that we cannot see through at all: the two crossed are as opaque to light as a brick wall, though both are as clear as glass! The beam transmitted by the first prism, is the one totally reflected out at the side by the second. At intermediate angles, part of each is transmitted.

Such a pair of Nicol prisms constitutes 'polarising apparatus.' One is mounted so as to go under the stage, either in a plain fitting or in the substage, and so that it can be rotated. The second one has to be somewhere above the slide. It is by some preferred mounted so as to be inserted, by a slot in the tube, over the objective, so that it can be rotated by a milled ring; by others it is preferred above the eye-piece. Both plans have advantages and disadvantages.

Stopping the light, by crossing the top prism, is only interesting. But if between the prisms we place a thin slice (such as most micro-slides or objects consist of) of any trans-

1 In a thick piece of Iceland spar we can see the two beams, (doubly refracted as immediately explained) by a spot on paper appearing double when looked at through the spar. 
parent substance which is doubly refracting (because more elastic in some directions than in others), there is an additional splitting up of the light, which produces appearances both beautiful and useful. The plane-polarised vibrations which pass the first Nicol, are now split up by the object into two, nearly equal if the two planes of vibration in the object are at angles of $45^{\circ}$ with the plane passing the Nicol. These are differently refracted, one being more retarded than the other in passing through the slide, but not visibly separated as in a piece of Iceland spar, because the object is so thin. Each of these two is, however, again resolved or split up by the first piece of spar in the second Nicol, or 'analyser' as it is called. So that we have now four beams, two of them vibrating together in each of the two analyser planes. The two in one of the planes, are totally reflected out to the side by the cut in the spar; the other two alone pass. Now, of these two pencils transmitted, and vibrating in the same plane, one is retarded more or less behind the other by the double refraction in the object. The discordance caused by this retardation causes interference between the two sets of waves thus forced to vibrate together in the same plane. The usual consequence of such interference, by retardation of one of two waves, is colour, as in a soap-bubble, ${ }^{1}$ sometimes only light and shade. Thus objects which 'polarise' display either the most gorgeous colours, or strong contrasts of light and shade, when examined by polarised light.

When, from excessive thinness, or weak doubly-refracting power, the phenomena consist of only shades of grey, it is customary to lay on the stage first a thin film of some crystal, usually selenite or mica, which itself shows a field of uniform colour by polarised light. Then a very slight

1 For full detailed treatment of the beautiful phenomena of Interference and Polarisation of Light, see my work on Light: A Course of Experimental Optics, 2nd ed. Macmillan \& Co. 
effect in the object itself, superposed above this, comes out more conspicuously on the coloured ground. Simple slides with one plain sheet of selenite are sufficient for most purposes, costing a couple of shillings. More complicated 'selenite stages,' which give a wide range of ground-colours, are prepared at higher cost.

The significance of polarised light lies in the fact, that nearly everything with any structure, not being homogeneous, is doubly-refracting. Glass itself becomes so if squeezed in a vice, the elasticity in one direction being affected; and by such compression alone gorgeous colour can be produced. Hence this appliance brings out or reveals structure, both in organic substances and in crystals, which in some cases cannot be so seen in any other way. In the study of rocks especially, polarised light is simply indispensable; all the different fragments of different minerals in such a conglomerate as granite, for instance, being brought out sharply in various colours. For study of rock-sections special patterns of polarising microscopes are constructed, always described in the catalogues as 'petrological' microscopes; and to such catalogues students intending to take up this branch of study must be referred.

Others add polarising apparatus for the beauty of the colour-phenomena alone. For such, a pair of prisms and a couple of selenites will be sufficient, and can be added from 1. upwards, depending chiefly upon size and mounting of the prisms, Iceland spar being now very scal'ce and dear.

41. Projecting or Lantern Microscopes.-These are constructed for the purpose of throwing upon a white screen immensely magnified images of microscopical slides, to be seen by an audience in the same way as ordinary lantern slides. At the suggestion of several members of the Royal Microscopical Society, about 1881 I began several years' special study and experiment with a view of improving such instruments (of which nothing efficient was then in exist- 
ence) in conjunction with Messrs. Newton \& Co. There were great difficulties, but the ultimate result was so successful, that the instrument thus worked out is now in use in all the principal scientific institutions in Great Britain, besides many in America and on the Continent, no other having as yet proved capable of similar demonstrations. Details would be of no general service in such a book as this, but it may be of some use simply to state generally what can be done. With only the oxy-hydrogen light, such an object as a flea or the tongue of a blow-fly can be shown crisply and brightly 12 feet long or more; and on smaller objects, such as certain scales of insects, the mouth-organs of a flea, or the barbed teeth at the end of an insect's sting, I am in the habit of using a power of 2,500 diameters, with a $\frac{1}{5}$ Reichert objective. With the electric light, which is far the best for this instrument, immersions are easily used, and 5,000 diameters and upwards obtained; but some practice and acquaintance with ordinary microscopy is required to do such powers justice, besides properly constructed compensating eye-pieces.

Smaller accessories will be best described in the next chapter.

\section{CHAPTER V}

\section{MICROSCOPE MANIPULATION}

Having got our microscope, according to our pecuniary means or general purpose, let us now consider how to use it.

42. Care of the Eyes.- This should be borne in mind, as injury might result from much work under certain conditions. The causes of injury are either (1) too fierce a glare of light, or (2) the vicious habit of always using one eye whilst screw- 
ing up the other. Too little light might also strain the eyes, but rarely occurs.

The light should always be subdued to what is sufficient and agreeable, either by contracting the iris diaphragm; or in some cases turning down the lamp; or by interposing coloured glass screens as mentioned presently.

It is much better to use each eye in turn if possible, keeping the other open; and this can usually be done after a little practice, the mind, as it were, not 'seeing' what is before the eye not in use. But if there is difficulty, it can be overcome by bending a piece of wire into two rings connected by a straight bit, as in fig. 43, the two rings being the distance of the eyes apart. One ring is covered with black paper, the other is made to 'spring' tightly on the neck or' cap of the

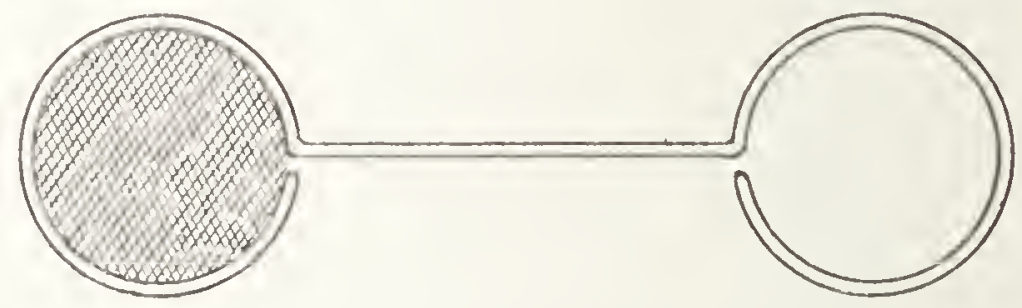

FIG. 43.-Eye Shade

eye-piece. The black screen will turn to either side for the unused eye. Screwing up the eye is most injurious.

A good binocular arrangement is principally valuable for its relief to the eyes, if expense be no object.

43. Three Great Principles.-From the beginning let a new student accept on the authority of all microscopists of experience, and keep present to his mind, three cardinal principles.

(a) Use no greater pouer than required by an object. Every novice has a tendency to magnify as much as possible ; the experienced worker does the contrary. If we can see all that is to be seen with a given power, we see it worse, and not better, by increasing. Of course a certain scale is necessary, and one observer can see much finer detail than 
another; I am personally heavily handicapped in this respect. But a beginner who possesses an inch and a $\frac{1}{4}$-inch will learn more and get on faster, if he does not use the $\frac{1}{4}$-inch at all till he has had several weeks' practice with the other; and in miscellaneous study, and even amusement, more work can be done with inch and half-inch than with any other powers.

(b) The eye needs edrucating as well as the hands. The microscopist will not at first see half there is to be seen with a given power, in a great many objects. If it were not so, an object would only need to be seen, and not to be studied.

(c) As much depends upon proper illumination as upon having the finest lenses and most sensitive focussing; in many cases far more.

44. Care of the Apparatus. - It is tiresome to be packing and unpacking, if much work is done; it is better to put the whole away under a glass shade, with velvet or cloth under the rim to exclude dust. The instrument itself should be dusted pretty often (gently and not flapped about) with a wash-leather. If working parts become stiff from cold or disuse, the very least touch of vaseline will generally suffice ; if not, or if anything appears loose, an average worker will do best to take it to an optician to be put right. When he has become familiar with every movement, he will probably be able himself to do this with the adjustments provided; but most novices had better let their instruments alone.

In regard to all lenses, take especial care never to touch a glass surface with the finger, or to breathe on it; either leaves a film of grease. If dew condenses on a lens on taking from the case, or in a warmer place, do not wipe it off, but pass the lens to and fro in the air to promote evaporation. An objective out of its box should be stood on its screwed end, with the front upwards, that no dust may enter the barrel; and for the same reason, when objectives are left on the instrument, an eye-piece should always be left 
on also, that dust may not fall down the tube. Objectives should hardly ever need attention, but eye-pieces must be cleansed every now and then from the dust that falls on them, whenever it begins to appear as specks in the field. Most microscope opticians will supply a chamois leather, carefully washed, and then cleaned with methylated spirit from soap and alkali. This should be cut in pieces and kept in a box, a piece being used when necessary, for glass only. The front lens of an objective may be cleaned with the same, and dust at the back can be removed with a clean camel-hair pencil cut square at the end, or a piece of the washed leather may be rolled up tightly and tied with thread, and then cut square across the roll with a sharp knife, to clean the back of a lens. If more be needed, the lens should be sent to the optician.

A water-immersion lens is dried with a bit of soft linen, washed and then soaked free from soap. An oil-immersion is first nearly cleansed with a dry bit of the same; then a piece is moistened with the tongue, and the moist linen will remove every trace. A handkerchief is often used at a pinch; but it is better to keep some cut pieces of clean soft linen in a collar-box, when such objectives are used. Care should be taken not to use too much of either immersion fluid; a single drop is sufficient. Lenses or slides should never be put away with oil on them, or it will dry into a hard varnish, which it is troublesome to remove.

45. Light.--This will be either daylight or a lamp. For moderate powers the first is most comfortable; for critical work with high powers the lamp must be employed, and often will be a necessity for the only leisure time available.

For daylight work, get near the window, so as to have the open sky and not an image of window-bars. Direct sunlight is, of course, to be avoided.

The precise kind of lamp is not material, unless 'critical' work has to be done, or unless photography is in question. The chief thing is a steady flame. An Argand gas-burner, 
I have found, will easily resolve the diatom Amphipleurce Lindheimeri, or give excellent images of bacteria. A shilling paraffin-oil lamp will do better in higher resolutions, and add intensity to even the above, using the edge of the flame for such work. A half-inch wick should be chosen. But for steady work and dense and crisp images, a proper microscope lamp is certainly better, and, for the highest type of demonstration, indispensable. Fig. 44 shows one of Baker's, sold for $17 s .6 d$., or in pine case for $21 s$., very similar ones being supplied by other opticians. The points about lamps of this type are, that the cistern is shallow, so that the flame can be lowered near the table to be focussed direct (i.e. without using the mirror) into the condenser of an inclined microscope; and, secondly, that the common glass chimney is replaced by one of sheet iron, in the side of which slides loosely a flat piece of glass, either white or coloured. Through this flat

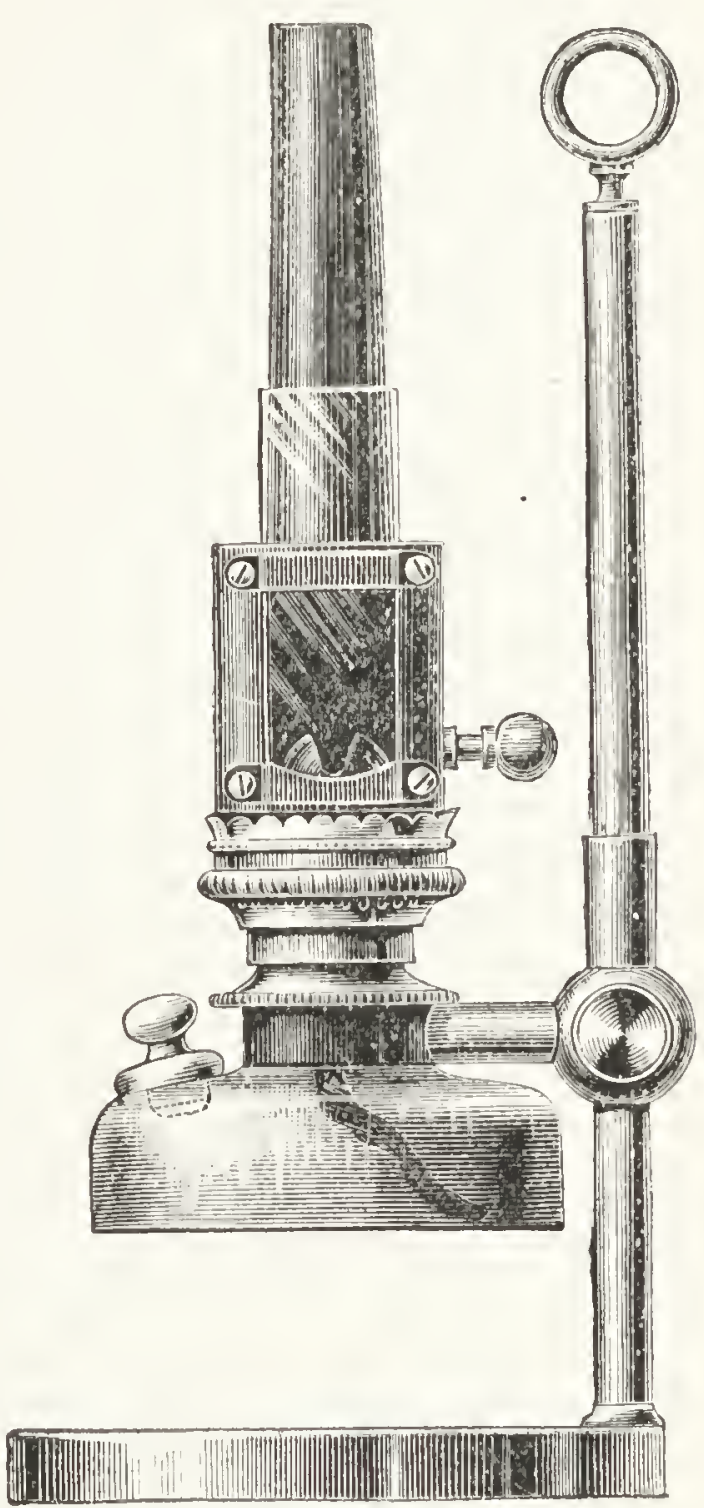

FIG. 44 glass, easily replaced if cracked, or toned to any colour, there is no distortion, and we have a sharper image of the edge of the flame. A metal reservoir is often used to bring the flame still lower, and rackwork to raise and lower it, and other refinements may be added; but a lamp as figured will answer all usual purposes well. 
46. Bull's-eye Condenser.-To avoid confusion with the substage condenser, or ' condenser,' or 'illuminator,' this is now generally called the 'bull's-eye.' It is a thick planoconvex lens, nearly or quite hemispherical, mounted with a

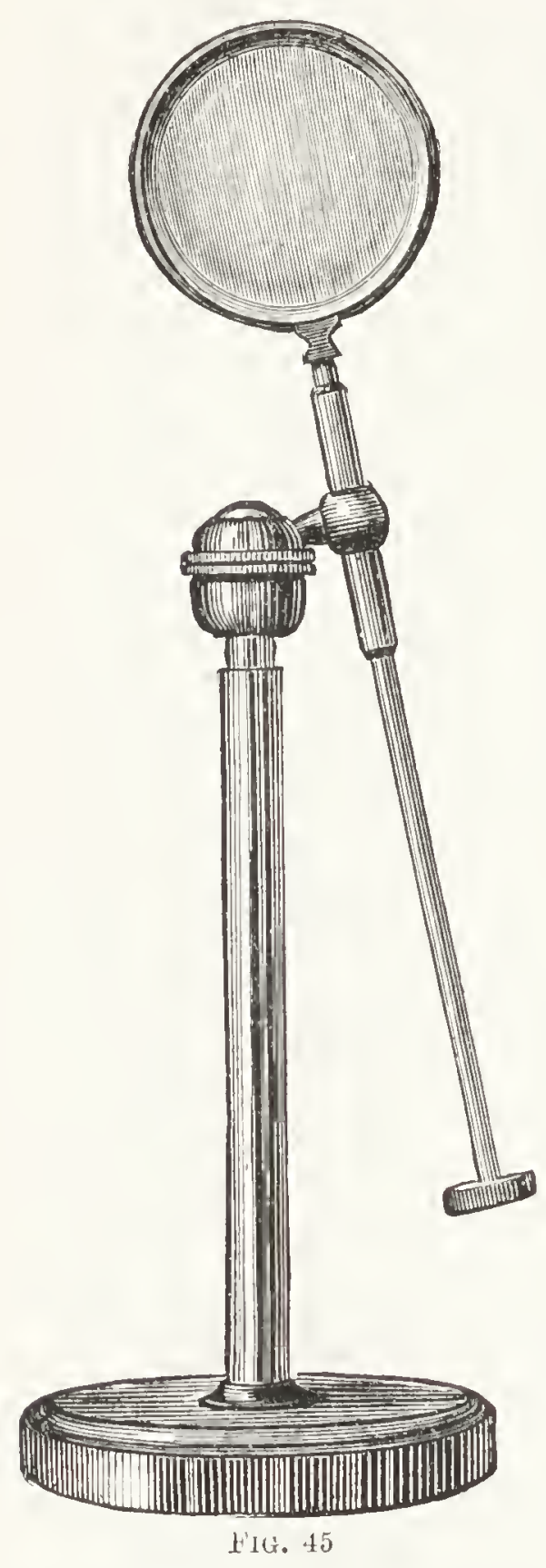
universal joint on a sliding pillar, somewhat as in fig. 45 ; but there are many patterns. It is better not so thick, i.e. less than a hemisphere, there being enormous aberration at the edge of a hemispherical lens. To diminish this a more expensive form is sometimes used, comprising two or even three lenses.

The bull's-eye is used chiefly for two purposes. One is to focus the light of the lamp or of a window (in this case some distance from the window is better) upon a solid object to be examined by reflected light. The more usual purpose is to obtain from the edge of the lamp-flame a nearly parallel beam of light about equal to the diameter of the bull's-eye and of the mirror of the microscope. It will be seen from fig. 6 , p. 17 , that this can be done by adjusting the bull's-eye at its focal distance from the flame. The flat side is turned towards the flame, and by covering the mirror with a piece of card or paper, the lens is easily adjusted so that a nearly parallel beam falls upon and fills the space occupied by the mirror. It is used in the same way when it is desired to throw a parallel beam of rays direct into the sub-stage condenser. 
47. Arrangements for Work. - The usual height of a table is convenient for most people, and enables one to look straight down a small-model instrument, when used upright and the stage flat, which is often necessary for ' wet' work. It may be rather low for the more comfortable inclined position, in which case a little wooden stage should be provided, like the lid of a box, of the convenient height; this will also be convenient for using light direct from a lamp on the table. A little bead round the edge of this, to prevent the foot of the microscope from being pushed off, is as well. When a lamp is in use, unless focussed direct, it is almost always most convenient to place it a foot or more to the left side; and if the chimney itself does not do so, all direct light from it should be screened from the eyes, and (unless wanted there) from the upper side of the stage.

For examination by several people, it is convenient to have a flat tray with baize on both sides, just large enough for the microscope to stand on with a sinall lamp in one corner. Then when an object is adjusted, the tray with all upon it can be pushed round to each person without disturbance.

48. Elementary Manipulation.-We will now suppose the simple Society of Arts microscope of fig. 27, with nothing but the mirror and wheel of apertures, with the inch (or lower power) screwed on, and some ordinary transparent slide on the stage, such as the proboscis of a blow-fly. How shall we attack it?

If near the window by daylight, with free sky or cloud illumination, the proper plan will be, after first inclining the instrument comfortably, to adjust the plane (flat) side of the mirror so as to throw the light straight up the optic axis. There is no particular focus to the mirror so used; but take the average distance. The sliding ledge on the stage being placed aright, get the object in the centre, and focus the objective with the coarse adjustment only; the fine adjustment should from the first be 'saved' as much as possible. 
Remember another point from the first. In working the milled heads of either the coarse or fine adjustment, or of a racked substage, take care to balance the pressure between finger and thumb. Never turn the head by bearing on one side, as one sees many novices do ; this would soon ruin any movement. Avoid also downward pressure of the whole hand, either on the tube or the stage, studying from the first a controlled, delicate, sensitive, balanced handling, free from force, or any shaking when the hand is removed. This delicate touch is essential to fine microscopy, and though it only developes by practice, and needs a good instrument to fully bring it out, it should be cultivated on the simplest stand from the first. There are many who can focus a high power with the coarse adjustment, more accurately than some do with the fine.

With daylight, the plane mirror will generally be about right as regards amount of light. If there is too much, try the smallest aperture in the wheel which fully embraces the object; if still too bright, a piece of white card laid on the mirror (preserving the same angle) will diffuse and subdue the light still more. On a dull day light is more likely to be deficient, in which case use the concave mirror. This latter, however, must be used at about its focal distance, which will be known nearly enough by its position when used with lamp and bull's-eye. If the light be now too great (there should be nice, soft, grateful light, enough to show casily what is sought, but no more), use the means mentioned on the next page. Never endure a disagreeable glare.

Suppose next that lamp-light is employed. For low powers, the flat of the flame should be turned towards the instrument; for the $\frac{1}{4}$-inch the edge will be better. The image of the flame should be focussed on the slide by the concave mirror, so that it is seen by the microscope in the same plane as the object, except with the inch and lower powers, when better effect is sometimes produced by bring- 
ing the mirror higher up, so that it focusses rather beyond the front of the low-power objective. There may very probably be excess of light from the lamp. This can be toned down by a blue glass shade, or using a smaller diaphragm; for often it is rather glare in the outer 'field,' than in the object itself, which tries the eyes.

But the pleasantest method of subduing the light is to have a circular piece of fincly ground glass cut to drop loosely in the stage aperture, upon the diaphragm plate just below, so as to lie slightly below the stage level. If a piece each be provided of plain, light blue, darker blue and neutral-tint glass, all the better, or the same effects can be got from one plain ground glass and a piece of coloured gelatine laid underneath. The light from the concave mirror sent through such a glass, suitably toned, will be found most grateful, and to give the sharpest image any ordinary objective admits of. It adds great comfort and pleasure to a vast range of work with the microscope. Even a piece of tracing-paper, dropped in the stage aperture, will give a great deal of the same effect, if nothing better be at hand. For the use of ground glass with higher apertures see $\$ 54$.

We will take next the $\frac{l}{4}$-inch power. With this it will be best to use the bull's-eye stand condenser to throw a parallel beam of light upon the concave mirror, which must be carefully adjusted to focus the edge of the flame upon the slide. Then bring underneath the diaphragmaperture which gives the best effect. Finally, with many objects sharper relief will be obtained by tilting the mirror a little, so as to get a slight obliquity of the light; but what is called 'central' light is to be preferred as a general rule. The direct cone of light from the mirror will sometimes be best with a good lens; but the performance (especially of cheap quarters) is generally improved by the ground-glass method just mentioned. Even with good lenses, many classes of objects are better shown this way than in any 
other, except with a first-class condenser; but with $\frac{1}{4}$-inch powers and upwards it is best to place the ground surface of a slip of glass in contact with the bottom of the slide itself.

49. Precautions with High Powers.-It is only too easy for the novice, especially if he hurries to use his $\frac{1}{4}$-inch at the beginning, to rack down a high power upon the slide, cracking the latter and perhaps injuring the lens. Even a cheap quarter will not be more than the thickness of a thick card from the cover-glass, and a better power will be nearer still. But there need not be the least danger. This is one chief merit of the slicting-bar shown on the stage in figs. 25, 32. It can be seen by the eye when a part of the slide is in the centre of the stage. Then with the nail of the left forefinger lift the front or top edge of the slide about the tenth of an inch. The objective can now be boldly but steadily racked down, and will be felt the instant it touches the slide thus slanted towards it. Moving the slide a little to and fro, some portion of the object will be found in the field, if only as a nebulous mass; and the slide, being gently let down flat upon the stage, is readily focussed without danger. The same procedure is followed on a mechanical stage, which has usually a free ledge for the purpose. It is to facilitate this use of the forefinger that the horseshoe stage (fig. 25) is preferred by some; but there is no real difficulty in lifting by the finger-nail on a solid stage. It is more difficult when the slide is pressed down by spring clips, as in fig. 28 ; but with a little careful practice at first it can be done even then. I, however, most strongly advise the sliding ledge for all plain stages, as an enormous facility and comfort in general work; while the spring clips on it, though usually turned aside, can be brought into play whenever required.

50. Searching a Slide.-In this, too, the sliding bar or ledge is of the greatest use. A slide often has many points of interest, which are by no means easy to find ' at random ' 
under a high power. With the sliding ledge it can be gone over as systematically as with a mechanical stage. Arranged first so that the top or bottom of the preparation is in the field of the lens, the slide is gently pushed, so as for the subject to pass completely from side to side. Then the ledge is pushed as much farther up or down the stage as will just bring another area the size of the field under the lens, and this stripe of the subject is again all passed under review, and so on; at the end all has been gone over, and nothing can be missed. If the stage and bar be 'divided' by lines (as in fig. 32), the situation of any point of interest in a slide, for that microscope, may be marked on the label by two figures, denoting the divisions, to which if the bar and end of the slide be again brought, the point sought will be in the field.

51. Nose-piece.-This appliance is of the greatest use for finding objects to be examined by $\frac{1}{4}$-inch or higher powers, or for rapidly exchanging the power; in fact, to the real worker (i.e. who uses his microscope to examine objects and not to exhibit his skill in producing superlative images of certain

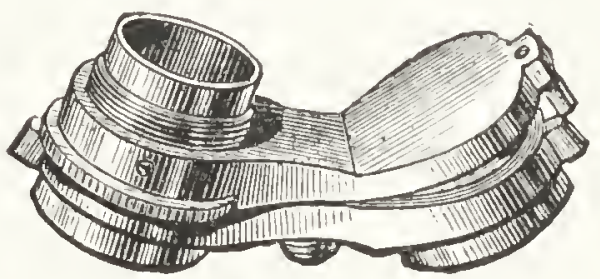

IIU. 46 selected subjects) it is indispensable. The double nosepiece (fig. 46) is the most useful, and will probably cost 15s. A triple one costs, say, 30s., but three lenses are rather heavy and rather in the way. The double one is shown on an instrument in fig. 32, and it will be seen at once how, the object being found and centred under the lower power, the higher can be rotated into place instantaneously. It is only necessary, when first using a nosepiece, to notice carefully how much (if any) the power should be racked back from the slide to make the movement safely. Years ago nose-pieces were very unsatisfactory, but are now made of very great accuracy and durability. 
Messrs. Baker have just introduced another and single nose-piece of great value. The female screw is divided into three jaws, worked by a small side-screw just like a centering chuck. When open, the objective pushes straight in up to the collar: then a turn of the side-screw grips the thread in an instant, and the least fractional turn of the objective makes all tight. It appears to work exceedingly well, and is sold for $12 \mathrm{~s} .6 \mathrm{c}$.

52. Use of the Condenser.-The reader will howevel, we hope, have an instrument with at least an under-stage fitting, or perhaps a substage, and somewhat better objectives than were considered in the preceding paragraphs. Even with the plain tube-fitting a condenser may be fitted, more or less like fig. 39. The condenser, or a plain lens or two, should be fitted to slide in the tube without great force, but so as not to fall down. The Abbe form is usually fitted so as to be in focus when pushed full up. A piece or pieces of finely ground glass, as already mentioned, may also be mounted so as to be capable of pushing up close to the slides for low-power work; the great comfort of this I know from an experience of years, since adopting it under the personal advice of the late Dr. Carpenter. With a proper substage there will be no difficulty; and, as already mentioned, any lens may be mounted for this in a plain boxwood flanged ring, until means permit of a regular condenser being procured. (Concerning this see \$39.)

Let us understand the principle of the substage condenser, which appliance we owe entirely to English microscopists and opticians. We have seen (\$ \&) that objects are seen or imaged by diverging cones of rays proceeding from every point in them, which are re-converged to corresponding points in the image. In objects seen by reflected light, or self-luminous, we have nothing beyond to consider; but in the case of transparent objects like most microscopic slides, examined by rays of light sent through 
them, most of which pass on straight after traversing them, it is different. The rays are now focussed by the objective in two characters: $(a)$ as image rays proceeding from the object; and (b) as original luminous rays from the lamp. The two must always have independent foci. The source of light (when used without mirror or condenser) is focussed near the back of the objective, while the object is focussed in the field of the eye-piece. This may not necessarily affect definition. But let us now suppose the rays from the lamp-flame focussed by mirror or condenser to a point a quarter-inch below the slide. As luminous rays, the rays then cross and diverge again in cones from that point; while as image-forming rays from the object, they diverge from it a quarter-inch higher up. It will be seen in a moment that such violent 'crossing' of the rays in these two character's must blur the image and 'flood' it with confused light. It is therefore necessary to make light-rays and image-rays coincide.

One way is to use the ground glass already mentioned. This is strictly correct and scientific; for the ground surface so 'scatters' the light, that there are no definite cones of luminous rays to be focussed as such, but the infinitely broken-up rays diverge from every point in the object, with no others to overpower them (\$54).

A second way, with the condenser, is to use this as in an optical lantern, so that rays from it (or from the concave mirror in the same way) converge to a point beyond the objective. The rays then never cross in their two character's; every ray sent through the object goes from it direct to some point in the objective, and we get a good image. This method is necessarily confined to low or moderate powers.

The third way is to focus an image of the light upon the object. The converging cones then become diverging cones, diverging from the same point in both characters; and in this way we get both the whole illumination and good definition 
with very high powers. This is the principal function of the substage condenser.

There are two methods of work, each of which may be carried out either direct or with the plane mirror. The direct method is simplest and easiest; the use of the mirror is more troublesome, but is often more convenient.

For direct work, the lamp is adjusted as low on the table as possible (unless the microscope tube for some reason is wanted to be horizontal, or nearly so), and about 10 inches usually (precise distance not material) from the back of the condenser, using the flat of the flame for larger objects and more moderate powers, and the edge for anything special with high power's. The flame must be in the centre, or optic axis ; and the easiest way to get this, at first, is to look through the empty tube, with nothing whatever between eye and flame. With a very little experience it will be easily done with all the lenses in place; but it is well for the learner to get from the first accustomed to the proper results of true central rays. It is very easy, with the rays at a small angle, to bring the image of the flame back in to the centre of the field by the centering screws of the substage, and for some purposes this may be even better, but such will be slightly oblique illumination: at present the light is to be central. Then the condenser is to be racked up to focus on the object, itself focussed and centered on the stage, with the low eye-piece, so as to show the flame in a large field. Then the flame image is brought into centre by the substage centering screws.

Should the plane mirror be used, see that its centre is in the optic axis, and then bring the reflection into the centre of the open tube as before. It is the plane mirror which is always to be used with the condenser.

The other way is to interpose the bull's-eye, and thus to supply the condenser with a beam of parallel rays. The plane side of the bull's-eye is turned to the lamp, and care must be taken that the lamp, bull's-eye, sub-condenser, and 
objective are all in one line, and the bull's-eye truly at right angles to this line. A piece of paper or card held across the bottom of the substage will show if a beam of fairly parallel rays is obtained, and the bull's-eye must be adjusted till this is the case. Then the object is brought into view and focussed; next the sub-condenser is focussed on the slide, and finally the centering screws, or perhaps a little movement of the lamp or bull's-eye, will centre the image of the flame. With the plane mirror there may be more trouble, but a little patience will soon effect the centering.

Working with parallel rays, the focus of the sub-condenser is somewhat shortened, and the angle of the outside of the cone increased. The illumination is also largely increased, and is too great except for high powers. The definition is not so good in delicate cases, owing to the uncompensated aberrations of the stand condenser.

53. The Illuminating Cone.-We have next to attend to the cone of light from the condenser. Suppose we have a quarter objective of $100^{\circ}$ air-angle, or N. A. 077 , and are using the 'achromatic Abbe' condenser of N. A. 1·0. That means an outside illuminating cone of $180^{\circ}$ in air, only obtained, however, when the condenser is in actual contact with the slide or has no focal distance. Therefore, no dry condenser can be aplanatic up to that angle, but the contral rays will focus the flame on the slide before the angle $180^{\circ}$ is reached. Place a dry 'test' slide of P. angulatum on the stage, and focus on it first the objective and then the condenser, centering the edge of the flame, and with the iris diaphragm closed to a small cone. Push aside the object and remove the eye-piece, and look down the open tube at the back of the objective; the iris aperture will show as a bright circle in the centre of the back lens (fig. 47). Open the iris and the bright circle will increase (fig. 48). This circle, when increased as much as will remain unbroken, will give the aplanatic cone of the condenser, and in this case should 
fill beyond three-fourths the diameter of the back of such an objective. In some cases the unbroken disc will be smaller, but may be increased a little by racking up the condenser; such enlargement will be a solid cone, but is not a truly corrected aplanatic cone. Racking up further enlarges the outer circle of light, which will enlarge till it far surpasses the back of the $100^{\circ}$ angle lens, but in doing so the bright disc 'breaks,' as in fig. 49, and only the largest unbroken disc of light at the back of the objective, as fig. 48 , can be used for critical work. In this particular condenser it will be about N. A. 65 , or an air-angle of $80^{\circ}$. If we were using an objective of only that aperture, its back would be just filled by such a cone, as in fig. 50. This is known as a full cone,

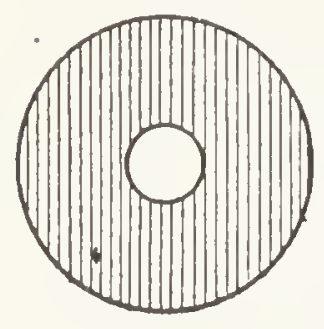

Fis. 47

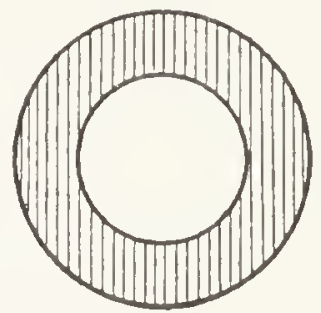

FI\%. 48

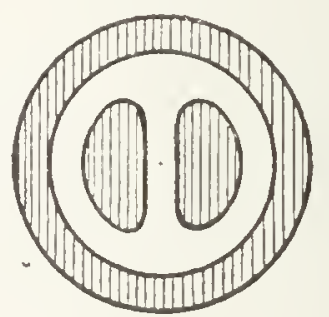

FIเง. 49

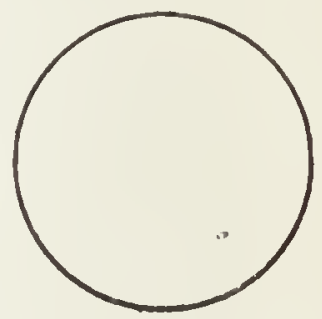

FIG. 50

and any greater angle than this cannot enter the objective at all to any good purpose, but can only blur the image by possible false reflection from the inside of the brass mount. ${ }^{1}$

In the present state of microscope optics, there is hardly a lens made that will bear a 'full' direct cone, even with a first-rate condenser: scarcely one will bear a cone over threefourths of the 'aperture,' and heaps of really good lenses will only stand two-thirds. The better the lens and the condenser, the more cone can be borne with advantage, provided the object itself will also bear it; but most objects will not. This depends chiefly upon thinness and opacity. With very transparent objects the cone may have to be cut down more and more, simply in order that the details may give (by their

1 The full elucidation of these details of the cone is mainly due to Mr. E. MI. Nelson. 
refractive differences) sharper shadows, which can be imaged. By the smaller cone we get this necessary light and shade, but at the same time we more or less thicken anc blur the image, or get diffraction-fringes round it.

Thus the microscopist has to employ different expedients, and sometimes to put up with one imperfection in order to fight another which is a still greater difficulty, and so on. This applies chiefly to higher problems, some of which have to be attacked in various ways before a result can be relied upon. It will suffice for most present readers to make some general study of the 'cone' as seen at the back of his ob. jectives when the eye-piece is removed, and gradually aim, by management of the illumination, at the best images, especially under high powers, which his apparatus admits of.

54. Full Cones from Ground Glass.-With good objectives, on all but thick or very transparent objects practically full cones may be employed, without the 'glare' of a full direct cone, by the use of ground glass combined with the condenser. The rays, focussed in this case upon the ground surface, are by it so scattered in every direction as to produce full cones from every point in the object, while there are no glaring oblique rays to blur the effect. Also there are no diffraction-fringes or spectra. Thus it was that, with moderate power, the fine hairs of the blowfly's proboscis were shown as fine at the tips as the lens was capable of (p.89). Laying now a ground-glass slip immediately under a test slide of $P$. angulatum, with any $\frac{1}{4}$ to $\frac{1}{6}$ dry lens good enough to bear Zeiss's highest compensating ocular, the diatom gives either black or white dot ( $\$ 60)$ with precision and ease. By grinding the back of the slide itself, rays enter the glass in cones far above N.A. 1.0, and (with a Reichert oil-immersion of 1.30 and Zeiss $\times 27$ ocular) Amphipleura Lindheimeri can also be dotted black or white quickly and certainly, with only an Argand gas-burner as illuminant. And bacteria exhibit beautifully. 
Such practical results cannot be against any sound optical theory, and their facility makes them advantageous in a great deal of work. ${ }^{1}$ At the same time, owing to imperfections still remaining in the outer zones of even the best lenses, and to the fact that many objects cannot yield light and shade with full cones ( $\$ 53)$, the carefully adjusted direct cone from the condenser will generally yield the best. results in very critical problems.

55. Oblique Illumination.-On racking up, a high-angled condenser gives its widest cone in a bright ring of light, the central pencil being of longer focus, and the rest worse than lost through spherical aberration. By placing various 'stops' in the fitting beneath, either the outer ring, and that alone, may be transmitted, or one or more pencils of light at extreme obliquity. This is often useful in two ways.

(a) When rays are sent obliquely across fine lines or striations in a very transparent object, the shadows are so increased as to give much more effect of light and shade in the image.

(b) When the object is so transparent that only a small angle or cone of light will give sufficient light and shade, this cone may not fill sufficient aperture of the objective to give 'resolution' (\$22). In this case we are dependent upon the diffraction spectra caused by the fine structure, to occupy and so use that aperture. But very fine striation may throw even the nearest pair of spectra from a central pencil quite outside the objective; if so, by using instead an oblique pencil, this pencil and one diffraction spectrum may come within the objective, and thereby using the outer portions of the aperture, give us resolution.

1 I was led to these results with ground glass used for full cones, in the course of $a$ theoretical and experimental investigation of Abbe's Diffraction Theor'y $(\S 23)$. Ground glass had been personally recommended to me years before for lower powers by the late Dr. Carpenter, but I do not think anyone had previously tested the results with high apertures, and especially with immersion lenses. 
Generally both modes of action come into play; and very often quite a slight degree of obliquity will marvellously add to the resolving power. With an ordinary $\frac{1}{4}$ objective, place a dry $P$. angulatum on the stage, and contract the cone with the iris till resolution just fails. The slightest tilting of the mirror will generally show lines. With an immersion lens the same may be easily seen with $A$. Lindheimeri. The resolution of $A$. pellucida in balsam is most difficult to effect with any other than a strong and very oblique pencil from an oil-immersion condenser and an oil-immersion objective. It is not alone the fineness of the striation- 92,000 per inch-as ruled lines on glass are resolved far more easily; it is the fact that the striæ are in clear transparent silex, and such phantom-like differences require artificial relief. No one need attempt this diatom in balsam till after long experience with the microscope.

But while smaller cones, and also oblique rays, are often necessary for the relief or contrast needful for resolution, this is never to be confounded with real truth of image. On the very contrary. Those black and white lines which a practised microscopist shows on Amphipleura pellucida, after infinite skill and pains, are utterly unlike the reality. $\mathrm{He}$ has produced by artificial shadow-intensification of almost phantom striæ, something he can see. A first-rate manipulator like Mr. Nelson can also produce its resolution with a central wide cone. The striation thus seen is far weaker, and by comparison nebulous and indistinct; hence it is often called 'inferior' resolution. But these very points make it probably a far truer image of the reality than the other. ${ }^{1}$

Fig. 51 represents some of the various stops used with a condenser for oblique illumination when used to give

1 These remarks refer to ordinary mounts. One of Dr. Van Heurck's mounts in arsenic (refractive index 2.0 or over) resolves easily, the great difference between the arsenic and the silex showing up the marking strongly. 
either relief, or resolution. The plain central stops shown in fig. 54 are often used, giving oblique rays all round the axis ; but the transmission of a central pencil as well, as in fig. 51 , A, often strengthens the resolution enormously; hence it

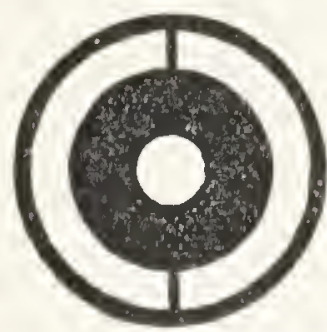

$\Lambda$

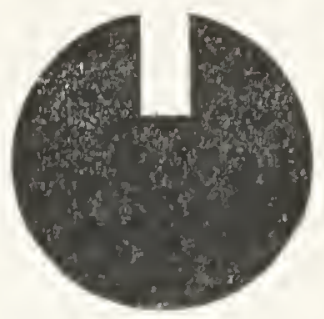

B

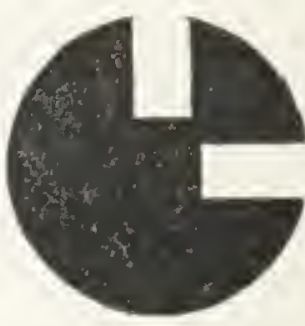

$($ :

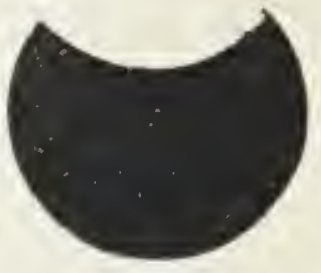

I)

FIG. 51.--Stops for Coudenser

is better to have most of the central stops pierced, with one central solid one which will cover the central pencil when required. The stop $B$ is largely used when one strong oblique pencil is needed, as in resolving A. pellucida: and D is another form which gives a single oblique pencil of greater

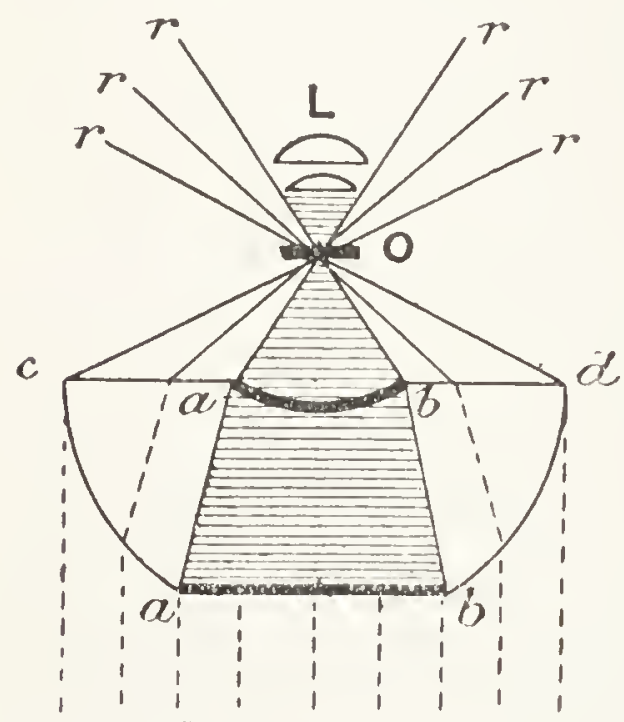

$R R R R R R R R R$

FiG, 52

width but less depth. The $\mathrm{c}$ stop gives two strong resolutions at right angles; and three similar notches equi-distant are also used.

56. Dark-ground Illumination.-This is another important use of the condenser, and is a very beautiful mode of showing suitable objects. The principle is best shown by the spot-lens (fig. 52), which was formerly used for low powers, and is often used still. It is a large condensing lens with central opaque spots $a b, a b$, ground out and blackened on front and back. Parallel rays of light $\mathrm{R} R$ being focussed on the object $O$, it is obvious that the central cone $a \circ b$ is destitute of rays. A similar cone from 
the object after crossing at o is also destitute of direct rays, and if this dark cone is as wide as the front of the objective lens at $\mathrm{L}$, no rays can pass into this lens through a plain slip of glass : there is a 'dark field.' But the object is further: illuminated by the outer zones of rays $c$ o $a, b o d$. These rays would not of course pass direct into the objective; but the details of the object scatter and reflect them to a great extent in all directions, and by these scattered rays the object, if adapted for this method, appears as if self-luminous on the black ground. The small silicious shells known as Polycystina (see Frontispiece) are best seen in this way; and some delicate details, such as the infinitely thin setæ of Floscules, can scarcely be displayed in any other.

It will be seen that a single lens can only suffice for objectives of moderate angle: for higher angles it was usual to employ Wenham's paraboloid (fig. 53). In this case parallel rays are not refracted as in a lens, but 'totally reflected' from the internal surface of a parabolic cone of glass $\mathrm{P}$, the truncated apex of which is ground and polished into a hollow semi-sphere, so that all the emerging rays pass through this at right angles.

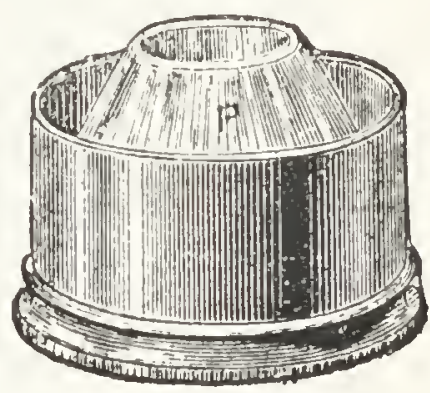

IFIG. 53 A central stop is mounted on a wire, which slides up or down through a hole drilled along the centre of the cone, and as this is pushed up towards the object it cuts off more and more of the central rays, or gives more of dark field, for a wider-angled objective. A good paraboloid will work up to an objective of $\frac{1}{4}$ or even $\frac{1}{5}$. Other devices have been employed. But a good condenser answers most purposes, ${ }^{1}$ inserting into the ring underneath it central

1 After careful comparative trials I do not consider it in all respects equal to a carefully worked paraboloid. Of the marginal zone of rays which alone are used in either case, much is lost in the condenser by reflection at the high angles. With the paraboloid, the same zone, being totally reflccted, suffers no loss. 
'stops' of various diameters (fig. 54) which can be cut out of blackened card if any special size be required. The condenser so modified becomes a compound spot-lens of high angle.

Suppose we have a few Polycystina to be shown in this way, we should proceed as follows. Arrange and focus the object in the ordinary manner, taking special care that lamp, condenser, object, and objective are all truly centered; and the same with stand-condenser if it is used. (The stand condenser will give a larger black field, and more light.) It only now remains to put in the smallest stop that seems likely to suffice, and rack the condenser up or down till the best effect is obtained; if that stop does not suffice, a larger
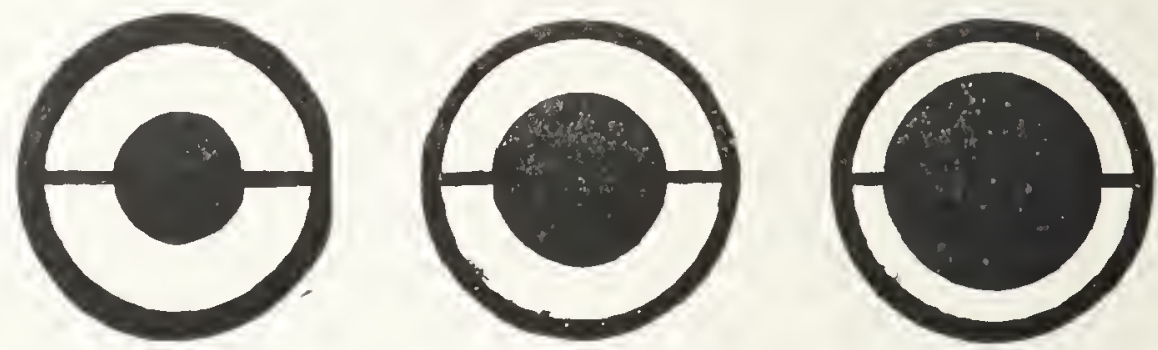

FIG. 54.-Stops for Dark Ground

one must be tried, the size of stop increasing with the angle of the objective. It is very difficult to get a dark ground with an objective over N. A. ${ }^{6} 65$ or air-angle $80^{\circ}$, but powers of more than this can be used by stopping down their back lens. That angle will, however, do all that any reader of this book is likely to require.

With low-power objectives, very fair dark-ground illumination can be had by simply swinging the concave mirror far enough to one side to fling all direct rays outside of the objective. These oblique rays act in exactly the same way, with the sole difference that, as they all have the same general direction, they only throw into very strong relief any striæ or lines or rows of dots at right angles to that direction. This may be useful, but such very partial effect has to be allowed 
for, and the interpretation corrected by turning round the object or rotating the stage.

57. Opaque Illumination. - A great many objects are best observed in this way, i.e. by reflected light. There are various methods available.

The simplest is to turn the microscope away from the window or lamp, and then to focus rays upon the object obliquely by the stand-condenser; or a bull's-eye condenser is often mounted so as to attach to the stage, or limb, of the instrument itself. Often no condenser is needed, but the direct light alone from behind the observer may be

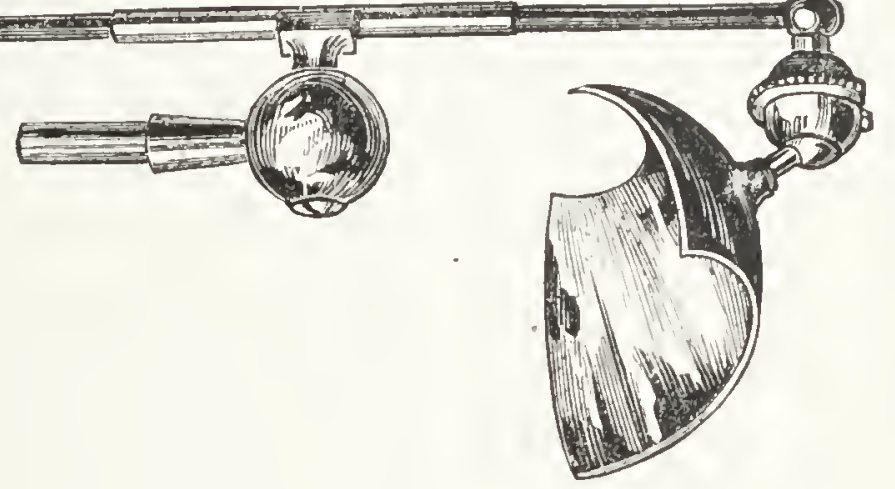

FiG. 55.-Side-reflector

sufficient. In most cases care should be taken that no light is thrown up through the slide from beneath.

The most general appliance used when more light is required is the parabolic side-reflector shown in fig. 55 . It will be readily seen how the two ball-joints allow of adjustment: the short stem fits into a hole in the stage or limb. The lamp being placed at one side, the rays are collected by the reflector and focussed strongly upon the object, obliquely from one side.

Another method, now too much neglected, is the Licberkiihn reflector, fitted so as entirely to surround the objective. The action will be seen from fig. 56, where the parallel rays from beneath the stage (parallelised by the stand condenser, and reflected by the plane mirror) are focussed by the 
reflector $c$ (here represented as screwed on the objective $b$ ) upon the object $d$. This object is here shown as held in a stage forceps $f$. No light should pass through the object; and this is often secured by mounting it upon an opaque black disc on the slide. These are generally much too

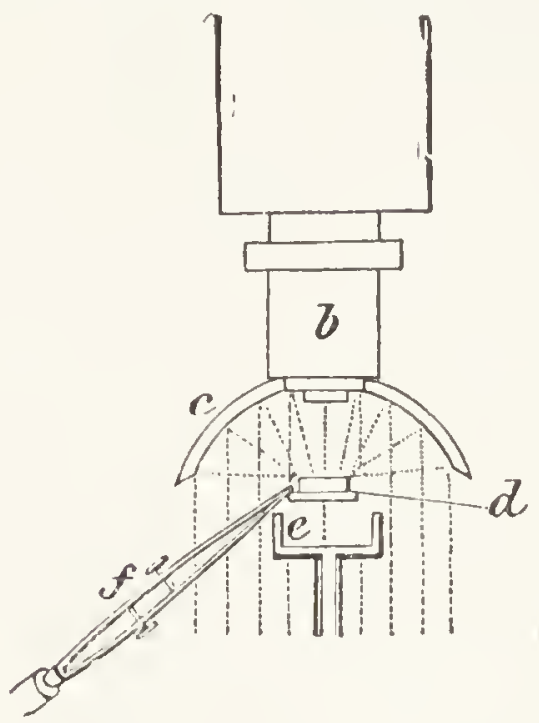

F [1: 56 large: the disc should stop any rays from entering the lens, but as few other rays as possible. A transparent slide may have a disc of black paper temporarily fixed at the back;

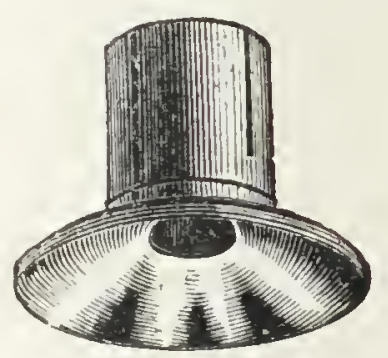

ГIG. 57

cr a better plan (necessary for objects held in forceps as in the figure) is to have one or two stops $e$ of different sizes (often called 'dark wells') mounted in the substage.

By having the barrels of several lenses the same size, a Lieberkühn made as fig. 57 may be fitted to slide on them, and be thus 'focussed' on a slide so as to work with all. Of course this is only possible within certain limits of focus.

58. Polarised Light.-Very few words are needed under this head; with the polariser and analyser in place, any object on the stage is at once seen 'by polarised light.' It is only necessary to say that the best effect is always with the prisms crossed, giving (apart from the object) the 'dark field.' The position on the stage will, however, affect the phenomena remarkably. It is best not to use a selenite beneath, unless it is needed; but if the effect is very faint, though visible, the selenite will often bring it out much more prominently. 
59. Combination of Methods.-Many objects can only be understood after examination in different ways. A large diatom, for instance, should be examined in the ordinary way, again with dark ground illumination, and also (if possible) with the binocular arrangement. Every one of these methods will add to our conception of the beautiful object. Two methods may also be combined at once. Thus, an object may yield further information when illuminated as opaque, yet with some amount of transmitted light as well. Dark ground illumination with polarised light, again, produces sometimes very striking effects. Mr. Nelson has pointed out the remarkable fact, that when a difficult diatom like A. pellucida is resolved, placing the analysing Nicol over the eye-piece in most cases appreciably intensifics the resolution. Some of these peculiar effects can hardly be said to be fully understood.

60. A Critical Image.-This is a very expressive term invented by Mr. Nelson to describe a sharp, perfect image of minute structure ; and every microscopist should try as soon as possible to educate himself to distinguish between such a one, only possible with good lenses and large cones, and the coarser images from inferior lenses and narrow cones. If he possesses good lenses, his own will teach him; if he only has the cheap $\frac{1}{4}$ sold with a three-guinea microscope, he should still try to learn this by the aid of some friend who possesses better. Two objects will suffice to mention here: a blow-fly's proboscis and a test slide (dry or in styrax) of $P$. angulatum.

The common narrow-angled $\frac{1}{4}$ will show all the main details of the proboscis, as described by the late Rev. J. G. Wood, very plainly. Unquestionably there is a high degree of pleasure and instruction to be obtained from what is so seen and known. The details will in fact appear thicker and blacker, and in some respects 'more magnified,' with such a lens. Fix now the attention specially upon the little 
pointed hairs that thickly stud the whole membrane near the tip. They will look, by the cheap lens, when we have done our best, quite plain, black, and thick. We see their number and that they are there. But such an image has missed the beautiful and delicate perfection of the minute works of God. Put on the better lens, of higher angle, and a good condenser, nicely adjusting all (or if a common condenser, using the ground-glass slip), and all is changed. The hair is now not thick, but has become thin, and goes off to a perfect point. The diffraction-fringes, or curious white nebulous lines, seen round the hairs before, have also vanished. We see the hair now as it really is. The fine, absolutely pointed little structure now before our eyes, is a 'critical' image.

Now take the diatom. The dividing cheap $\frac{1}{4}$-inch will not resolve this at all, but we suppose an objective that will do it easily, which can be had for 25s. Use light from the plane mirror only, with a small stop in the substage, or as small cone with the condenser as will do it. The diatom, now, looks very 'black and white' indeed. But it will be found that all sorts of images appear as the objective is focussed up or down; it is hard to judge which is right. Try it now with a better lens, and with a large cone carefully adjusted, or a fairly large cone and the ground glass. There are now but two appearances possible-either white spots or black dots arranged in sixes and sharply focussed, the one a shade nearer in focus than the other. These are true and 'critical' images, the two effects being accounted for by well-known laws of physical optics. The black shadow contrast is not nearly so strong; but the image displays the delicate details, sharp and true.

There are higher lessons to be learnt with experience; but these will suffice for the present reader, with the remarks in $\$ 61$. 
61. Collar Correction.-The principle of this has been explained in Chapter II, § 16. The student will only need it as he begins to use rather wide-angled dry lenses, and to understand the difference between a blurred and critical image. When that time arrives, a few simple remarks here may save him some time and trouble.

First recall the principles concerned. An objective requires 'correction'; and if any objective is perfectly corrected for an uncovered object, it becomes over-corrected when the object is covered by a glass; the more orercorrected the thicker the cover-glass, or the glass and layer of balsam or fluid (if mounted in such). To compensate or counteract this, the objective itself, or else the whole optical combination, has to be made more or less under-corrected for the uncovered condition. In less degree, if an objective is corrected for a given cover-glass, it will need to be made more under-corrected for a thicker cover-glass.

This under-correction may be effected in two ways, but which are really the same in principle. The first is, by the 'collar,' to bring the front and back combinations of the objective itself nearer together, which in modern objectives means turning the collar to the right or to the higher mumbers. The other is to shorten the tube-length, or bring the eye-piece nearer the objective; this, to be effective, has to be done to a far greater extent. But it is really the same thing in principle, since (as has been pointed out in \$10) the front portion of the eye-piece in principle belongs to the objective.

Thus fixed objectives of high class, such as Zeiss's $\frac{1}{2}$-inch apochromatic, have to be 'corrected' by lengthening or shortening the draw-tube. The old directions about getting more power by 'extending the draw-tube,' can only apply to lenses very moderate in angle and quality; a 'critical' image can only be had, with a fixed objective and given cover-glass, within very narrow limits indeed as regards length of tube. Hence, again, collar correction may come 
into play on account of tube length; and though it is not usual to provide so much of it, an apochromatic $\frac{1}{4}$ was made for me by Mr. Powell which could be used for either the 6-inch or 10-inch tube.

Next for collar-correction in practice. Good slides to practise upon are a dry $P$. angulatum and a Podura scale. Suppose it is the diatom, and the lens a really good $\frac{1}{4}$-inch. First of all, see that the collar works easily. Secondly, get thoroughly into the mind the fact, as an instinct, that to turn the collar to the right (looking up the tube; from the operator's position behind the instrument it will be seen to be to the left) is to close up the lenses for more under-correction. Thirdly, without any attention to correction, practise a little in working the collar a bit, whilst keeping in focus on the slide with the other hand.

This is just the mere handling of the thing; and now we can attack the diatom. Find one of the flattest, and focus one edge. Then alter the focus a little, up and down alternately, taking great care to move the milled head equally each way. Both ways the outline will open, or enlarge into a nebulous coma, from the focal image; and it will probably be seen that the enlargement is greater (for the same motion) one way than the other (Wenham). If it is greatest when the objective is drawn back, open the lenses; if greatest when the objective is focussed down below the true point, close the correction (collar towards higher numbers). Do this till the expansion appears about equal both ways; the correction will now probably be nearly correct, and with some observers may be quite so.

Next examine the image itself, and see if the small markings appear in the same plane as the grosser outline. If they appear distinctly above the gross image, the correction is not right yet. Work a little more till both seem to focus nearly, at all events, in the same plane. The correction will now be very near, possibly quite so. 
But finally, the observer must learn to judge of the sharpness and density of the image itself. Getting as large and good a cone as he can, or as the lens will bear, and testing with the highest power eye-piece he has which it will stand, let him now study on the diatom or Podura scale the sharpest marking. On the diatom he should get at will, with a very slight change of focus, either an array of sharp little white discs, or in their place black dots, and no others ; working the collar till both are as sharp as possible. On the Podura, the white exclamation mark on the top and within each black one, should also be as sharp and distinct as possible. Bacteria should appear cleanly drawn and thin.

When this is attained on such representative slides, the student will have learnt sufficient of collar-correction for his present purposes; anything more that he needs will gradually come of itself.

\section{CHAPTER VI}

MICROSCOPICAL DRAWING, MEASUREMENT, AND PHOTOGRAPHY

The genuine student will occasionally require to draw what he sees, and may also desire to measure either the size of the objects or the magnification of his images. Both operations are simple and easy.

62. Drawing.-This is performed by some modification or other of the camera lucida. The simplest, cheapest, and easiest for a beginner to use is that known as Dr. Beale's neutral-tint reflector (fig. 58). The usual cap of the eyepiece is removed, and replaced by that in the figure, outside which is a simple mount which supports a piece of transparent neutral-tinted glass at an angle of $45^{\circ}$. The microscope is adjusted with its tube horizontal, and, if the stand 
be small, should be supported on a book or box, or the stand suggested in $\S 47$, so that its centre is ten inches from the table, on which the paper is laid. The image is

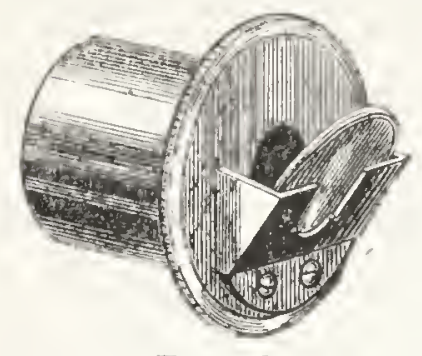

FIr. 58 then reflected upwards to the eye looking straight downwards, and which also perceives the paper and pencil through the tinted glass. This apparatus costs from $5 s$. to $7 \mathrm{~s} .6 d$., the latter sum procuring several glasses of different shades.

Various more costly prism arrangements are also in use, and Abbe's camera lucida has a reflector which enables the microscope to be used at any angle. Such arrangements will be found figured in first-class catalogues, and need not be particularly described here. Some are rather difficult to use ; but it is singular that one observer will find most easy, the very one which another finds most difficult. Only one practical remark need be made, viz. that success depends much upon the relative brightness of the image as seen on the paper, and of the paper and pencil themselves. Hence a separate lamp to illuminate the paper is often desirable, and upon increasing or diminishing the light of either, comfort and success may often depend.

63. Measurement of Size.-The real sizes of minute objects are easiest measured by the aid of a stage micrometer, costing about $5 s$. This consists of a line ruled on a glass slide, and divided by cross lines into small spaces, usually the $\frac{1}{100}$ and $\frac{1}{1000}$ of an inch, or $\frac{1}{10}$ and $\frac{1}{100}$ of a millimetre. The object being conveniently adjusted, its leading dimensions are sketched on the paper (a mark or two usually sufficing). Then it is replaced by the micrometer; and the image of the small spaces being made to coincide, gives at once the dimensions of the details of the object.

Another method is to employ an eye-picce micrometer, containing a glass scale in the field of the eye-lens, and which is consequently seen in the same plane as the image. 
The stage micrometer is then imaged (of course this should be done with the same power and tube-length employed on the object) and the value discovered of say one or more $\frac{1}{100}$ ths of an inch, on the larger divisions of the eye-piece micrometer. Then the dimensions of the object are known by comparison. Cheap eyepiece micrometers on circles of glass are sold (fig. $58 \mathrm{~A}$ ), which can be dropped into ordinary Huygenian eye-pieces. An eyepiece with micrometer fitted is much more expensive.

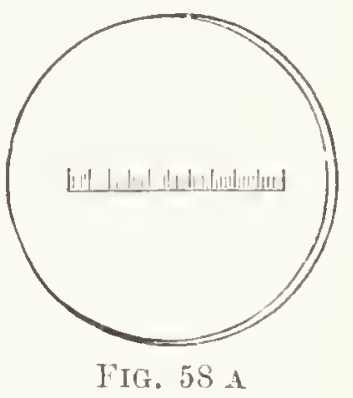

64. Measurement of Magnifying Power.-This also is simply effected with the aid of a stage micrometer and the camera lucida. The paper must be placed at the proper distance of 10 inches ( $\$ 1)$ from the neutral-tint glass. Then the image of the micrometer is focussed and dimensions sketched on the paper. The most convenient division so sketched, has then simply to be measured to give the magnification. Thus, let us suppose the division known to be $\frac{1}{100}$ th of an inch measures when drawn $3 \frac{1}{2}$ inches, it is obvious that the magnification is exactly 350 diameters.

65. Photography. - The highest class of photo-micrography demands costly apparatus, the very finest lenses, and the highest manipulative skill, and to enter into it is out of the question in these pages; we can only give a very few hints towards success in earlier and simpler attempts, which may, perhaps, lead on towards more ambitious work. In that case, elaborate treatises on this special subject are accessible to the student. The delicacy of such work as highly magnified photographs of the more difficult bacilli may be gathered from the fact, that it is hopeless to attempt it except on the solid ground, in situations practically free from the vibration always present in the crowded parts of large cities.

Micrography having additional difficulties of its own, the 
operator should first of all have sufficient ordinary camera practice to master the ordinary photographic details. $\mathrm{He}$ should learn to judge by the behaviour of his plates whether the exposure has been correct, too long, or too short; and how to 'humour' development according to these circumstances. It is also advisable that he should become familiar with the use of the quicker plates, and especially of some of the best brands of isochromatic plates, which latter give far better results with many insect subjects, or with others of difficult colours.

The microscope for early work need not be expensive; but must be steady, capable of being balanced with the tube in a horizontal position, and should have a focussing and centering substage. Some amount of skill in proper illumination and display of the objects to be photographed must also be acquired; for if the visual image be not good, it is impossible for the photograph to be so. These points are almost self-obvious; but observation proves that dozens of plates and much time are absolutely wasted in efforts that cannot yield good results, for want of attending to them.

When thus ready for actual microscope work, there are two general nuethods of operation; but in either case, with most magnifications it will be needful to provide a thick and solid base-board four to six feet long, which is all the better if provided with strips on the edges of the top surface, to form a trough or slide for the apparatus to move in, easily but in line. Along one edge of this, in two or more eyes, should revolve easily a brass rod or tube, with a pulley fixed on it, round which and the milled head of the fine adjustment passes a cord or tape. Usually it will be a tape; but when a microscope is intended for photography, a groove is often turned in the milled head, and a cord used in the groove. The purpose of this is, that when the image which has to be focussed is too far off from the microscope for the operator to reach the fine adjustment with his hand, 
it may be worked by turning the rod, which has a milled head on the end. A very solid table should be used, on the most solid floor that can be found; but Dr. Charters-White says that on such a table he has found that vibration is largely prevented by supporting the base-board on two piles of unbound magazines.

The first method of working was recommended some years ago by the gentleman just quoted, and consists essentially in placing the microscope and lamp themselves in a box open only at one side, which also can be closed entirely by an efficient black curtain; and with an aperture in one end opposite the end of the microscope body. Over the lamp must of course be also a chimney, which must be light-tight during exposure. The aperture through which the microscope protrudes is easily made light-tight by fixing outside a short tube, round which and the microscope tube a loose tube of black velvet or cloth is kept tight by indiarubber bands. Outside the box is provided any kind of moveable stand to carry the focussing screen and plates, which can be adjusted at any distance from the microscope required, according to the size of image.

Large objects with low powers may be focussed on an ordinary ground glass; but for minute detail this will not be sufficient. In some cases it answers well to have white paper pasted on the front side of a glass plate, and look at the image, projected as on a white screen. One advantage of this method is that no focussing rod is needed, as the fine adjustment can usually be reached, while getting a critical view of the image on the white paper. But the usual plan is to use a glass plate, on the face of which (corresponding to the film in position) are ruled some fine lines, and to carefully focus these and the image together with a 'focussing-glass.'

The procedure with this sort of apparatus is pretty obvious. The lamp, substage condenser, and microscope are 
carcfully centred, and the illumination carefully adjusted in the usual way, till the very best image possible is attained, and the screen is so adjusted as to get the image the required size and sharply focussed. Often it will be best to project it direct from the objective alone, at several feet distant. In other cases an eyepiece may be employed, at a shorter distance. The best results of all, for high powers, are obtained with 'projection' eyepieces, but these are unsuited for low powers, unless apochromatic.

Thus far the side of the box is left open to give light in the room: now the curtain must be closed and the whole left for ten or fifteen mimutes. All will then probably appear out of focus, owing to the expansion of the metal by the heat inside the box. This must be readjusted: and not until the image appears to remain stable should exposure be attempted. Then the plate is placed in position, and a loose cap over the microscope end; the shutter removed (all being now in total darkness), and finally the loose cap removed and the exposure given, by watch. Correct exposures must be learnt by experience; but a pretty good average exposure to start trials with, at say 100 diameters, with ordinary lantern plates, may be 20 to 30 seconds.

Many good ordinary objectives may be made to give fine definition, equal to that of most apochromatics, by interposing a sheet of Chance's 'signal-green' coloured glass. The time of exposure is, however, increased considerably. Other photographers have obtained splendid results from various coloured screens and isochromatic plates. These last give the best results with the more difficult insect subjects.

With low powers, the ground-glass method of illumination will often be found of great use. With high powers it absorbs too much light, and reliance must be placed on carefully-adjusted central cones, as large as the lenses and the object will bear. In all cases the photographer should be careful to stand or sit still during exposure, as walking 
about the room would probably spoil the image by the vibration so caused.

The other method of work is to place the lamp and microscope free on the base-board, and an ordinary camera with its dark slides at the other end, removing its lens, and making a light-tight connection between its tube and microscope, in the way already described. With the usual short cameras, an eyepiece will be necessary to get a sufficient magnification; but additional bellows, up to several feet in length, can be obtained for a small sum. To use these the focussing-rod will of course be necessary. Fig. 59 shows such an arrangement in conjunction with the simple stand

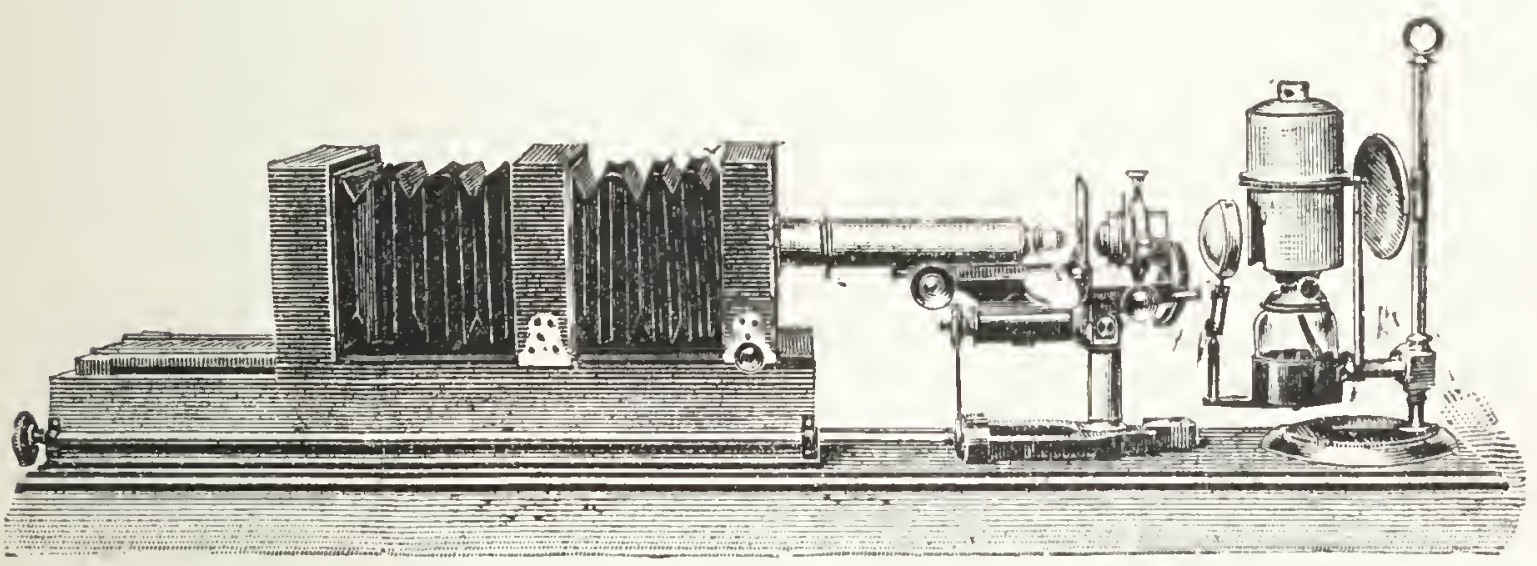

FIG- 59.-Microseope and Camera

illustrated in fig. 31, with an ordinary lamp, and bull's-eye to supply parallel rays. Mr. E. M. Nelson has devised and described a simple arrangement of light-tight boxes open at both ends, blackened inside, and fitting into one another, with blackened internal flanges. With these a camera of any length can be built up, according to the number of boxes used end to end, which could be made by any amateur carpenter.

This latter method of work (i.e. with a camera) is generally preferred by practical men. It allows the room itself to be well lighted throughout, and gives facility for using a lime-light jet, or any other powerful method of illumination. 
And the apparatus becomes far less heated than when itself enclosed in a dark box, though it will be found that even the proximity of the lamp will affect the focussing somewhat, until the whole has been some time in position.

The majority of good modern objectives are fairly well corrected for photography. Nearly all are corrected and sharp in focus, if a signal-green glass is used.

In photographic work, it is of the utmost importance to prevent all 'flare' from bright reflected light. Some otherwise good objectives reflect a great deal of light from bright brass round the edges of their lenses; all lens mounts should be blackened. The larger in diameter the tube of the microscope is, the better; and this should be carefully blackened inside with black velvet; no mere varnish is ' dead' enough to prevent all reflection from the sides of the tube. Or another plan as good or better, is to cut several discs of card of a size which will jam or stick anywhere in the tube; cut apertures in the centre, of rather different sizes, the largest slightly smaller than the field-lens of the eyepiece; and make them dead-black with suitable varnish or Indian ink. The smallest is pushed two-thirds down the tube, and two others spaced so that the last and largest is about an inch from the bottom of the eyepiece. These side-stops catch all the surplus side-rays. The bellows or cameraboxes should be protected in the same way, square-shaped stops being easily placed at intervals along the camera.

Many plates produced are the veriest rubbish (unless of some rare or unique object, of which any photograph may be better than nothing). It should be understood that in this branch of photography there is as much room for taste and manipulative skill as any other. Of some objects the sharpest definition and the sharpest contrast in black and white may be desirable; but in others good half-tones, harmony of shades, and general effect are as important as in other subjects. Exquisite slides can be produced of suitable 
objects by the 'dark-ground method' of illumination. Then the thickness of the microscopic object has to be considered. If very appreciable, only certain portions can be 'focussed' at one time by a high-power objective; and if tolerably high magnification is required, it must be obtained by using a power low enough to get 'depth of focus,' and making up the magnification by either an eyepiece, or greater distance of the plate.

These difficulties and refinements should not be encountered all once, but the first attempts made upon easy and flat objects, of which a flat image can be at once obtained. Sections of the stems of plants, and the wings of some insects, are very good objects to commence with. If serious and systematic work is then determined upon, the student will do well to obtain one of the detailed manuals concerning this branch of microscopy.

\section{CHAPTER VII}

manipulation, PReparation, MoUnting, and selection of OBJECTS

WhoLe treatises have been written upon this division alone: we must be content with those elements suitable for a beginner.

66. Natural 0bjects.-Very many objects are well worth examination in their simple, natural state. Small flowers whole; their parts; insects and parts of these-all such will well repay observation as found. For this purpose an indispensable appliance is the stage forceps, fig. 60 . The stem of the forceps fits into a hole in one corner of the stage, or the limb of the microscope, as most convenient; 
and the slide, combined with the universal motion, enables a small object to be placed in any position, angle, or direction desired.

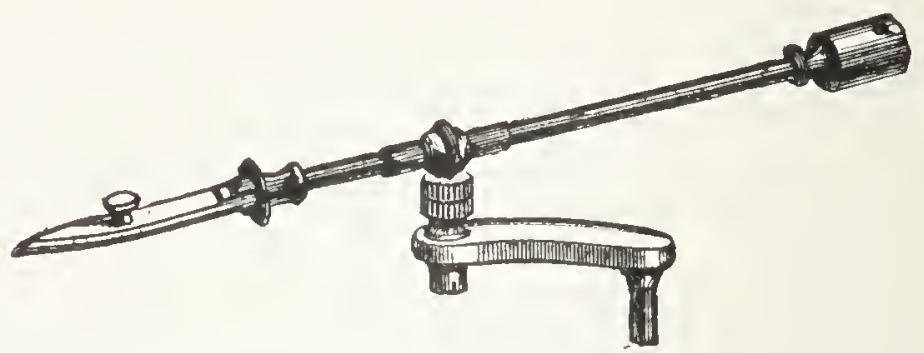

FiG. 60.- Stagc Forceps

Most objects examined in this way are illuminated as opaque objects, by the bull's-eye alone; but the parabolic reflector ( $\$ 57)$ or the Lieberkühn can also be used. Some may be transparent, such as the wings of many insects; and for these the concave mirror or condenser may be used as usual.

67. Live 0bjects.-One way of examining live insects has already been described on page 42 , but only serves for the head or mouth organs. Another method is more generally
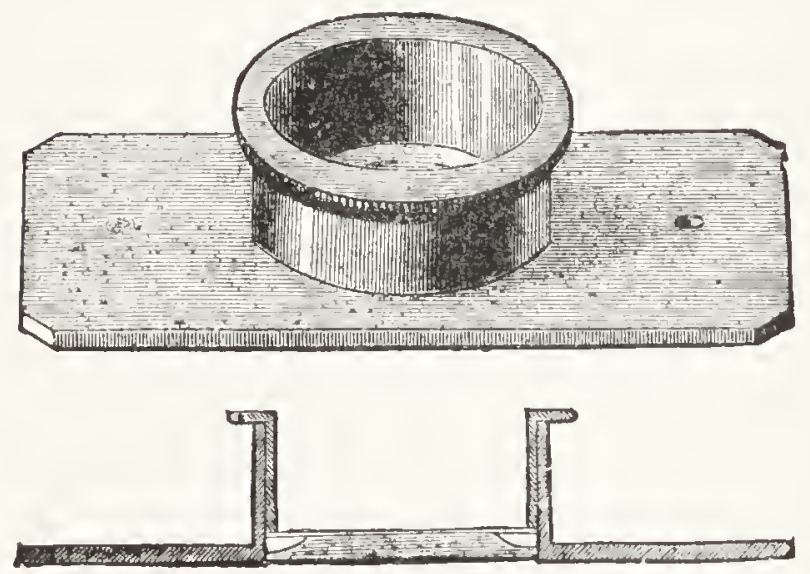

Fig. 61.--Live Box useful. A flea or other insect may be stupefied by the vapour of alcohol, ether, or chloroform, and while unconscious attached by a particle of gum on its side to a very small disc of paper gummed on the centre of a glass slip. When it recovers, the movements of any part can be well seen, by aid of the parabolic reflector or a Lieberkühn.

Another very usual method is to einploy the simple apparatus called the live box (fig. 61). On a slip of brass is fixed a short wide tube, in one end of which is mounted a 
circular plate of glass. Another short tube sliding a little stiffly, either in or outside the other, has mounted in one end a thinner plate of glass; thus with care the two glasses can be adjusted at any distance desired, so as slightly to compress an insect or small animal between them. This apparatus is made either with the glasses at the bottom of the tube, or at the top, for convenience, and costs 5s. to 8s. Mr. Rousselet's form is best, in which the glasses are at the bottom of a much wider cell, so that an objective can focus anything up to the very margin of the glasses. More perfect contrivances are made at higher prices, with which the pressure can be more precisely adjusted.

68. Pond and Marine Life-Living objects in water are handled in various ways. Infusoria, and many Rotifers, are
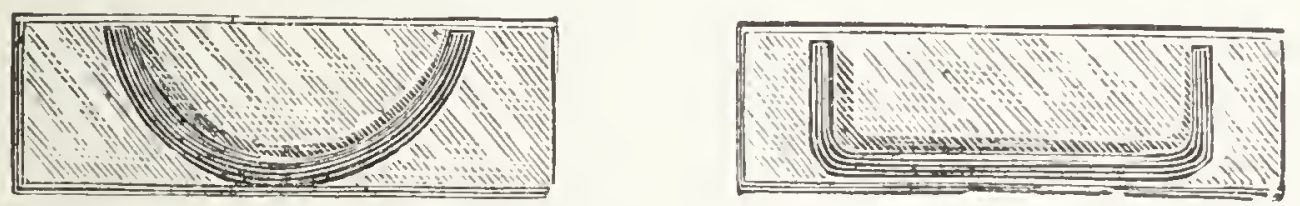

Fig. 62.-Troughs

best examined by simply putting a drop of the water containing them on a glass slip, and a loose cover-glass over, which is held on by capillary attraction. If so large as to be crushed by this, a filament of wet cotton, or of some confervoid growth, will keep off the pressure, and yet confine the movements of the animals; or it is very usual to employ the live box, enclosing now a drop of the water between the two glasses.

But the majority of creatures will require glass troughs, the smaller of which, resembling fig. 62, can be obtained from 1s. each upwards, and consist of cells cemented on ordinary $3 \times 1$ glass slips. Thinner cells, and with thinner front glasses than can be generally purchased, will, however, often be required; and I have been in the habit of making mine. Provide half an ounce of cover-glass, about $1 \frac{1}{4} \times \frac{3}{4}$ inch, for 
the fronts of the troughs. For the thickness, or wall, half of an ordinary vulcanised-rubber ring of suitable size will do; or for very thin troughs, a wall cut out of thin card. Either may be cemented to the slip and the front quite securely, by the ordinary balsam and benzol.

Another good way is to use glass slips with concave cells ground out of the centre; these provide a certain depth of liquid, over which a cover-glass can be placed. Such cells are sold in great variety, and also with subsidiary cells and grooves, so arranged at their sides that a current of aerated water may be maintained if necessary. These devices are known as 'life-slides,' and after a little experience the manner of use will suggest itself. A great variety are figured in Beck's larger catalogue.

For larger animals, Polyzoa, \&c., large troughs are sold, of all sorts of shapes and sizes. When a trough is rather deep, and an animal has to be kept near the front, this is done by wedging up a slip of glass behind it. Marine life attached to weeds requires rather a large trough. What is called 'Botterill's' trough consists of two glass slips, with half a vulcanised-rubber ring pinched between them, or two layers if required, by means of two outer plates of vulcanite and set screws. Personally, I have found made troughs most convenient.

Vegetation, with or without life attached, is best arranged in a trough with a slender pair of forceps or needles. Separate animals may be withdrawn from the water they are kept in by a 'dropping tube.' The tip of the forefinger is held tightly over the top end of an empty tube of suitable size, and the lower end being brought over the animal, when the finger is lifted the water rushes up and carries the creature with it. Replacing the finger, the whole is lifted out, and removing the finger again will deliver the animal in the trough or on a slide. But by far the most convenient implements are the 'filler's' sold with stylograph or fountain pens; if 
a few are at hand with different bores at the point, small animals can be captured and again expelled with the greatest facility.

69. Dissecting,--The minute details of many objects have to be carefully separated in order to be examined. The tools needed for these manipulations consist of fine pointed scissors, forceps (fig. 63), needles for teasing-out fine struc-

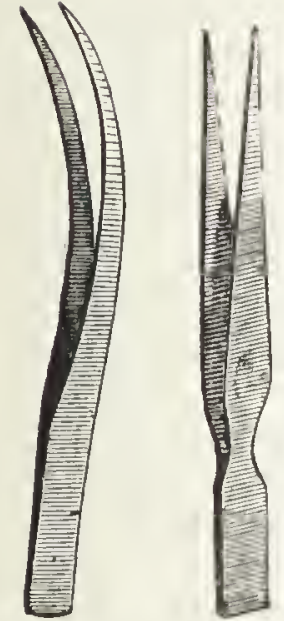

FIG. 63.-Foreeps

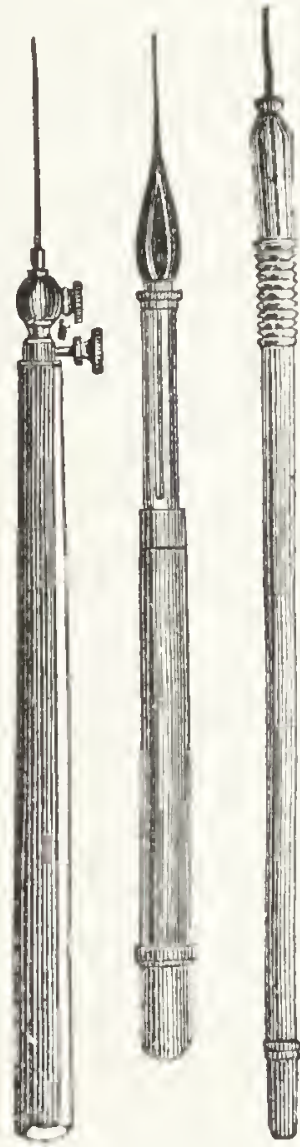

Fig. 64.-Needles

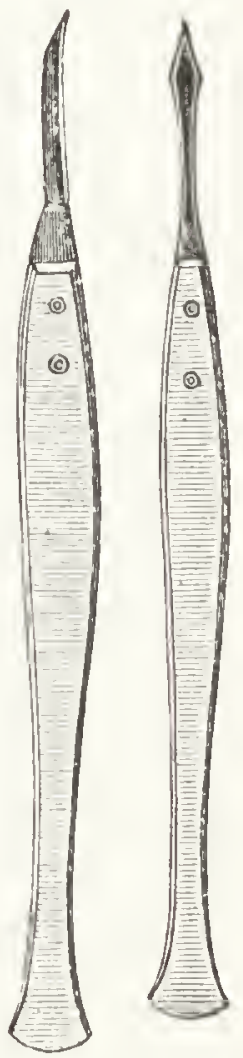

Fra. 65.-Knives

tures (fig. 64), and dissecting knives (fig. 65). Forceps are required both straight and curved, and are usually of metal; but for wet work I prefer them with ivory tips. Fig. 64 shows three ways of mounting needles in mechanical handles. But it does just as well to provide some cedar handles, as used for camel-hair pencils, to bind one end round with thread, and having made a hole in the end with the point of a needle, to push the butt-end firmly in with a pair of pliers. 
There should be several straight needles of various thickness, one or two curved, and one or two bent quite to a right angle and into hooks. Knives need no description. A few camel-hair pencils, with perhaps one or two sables for their greater stiffness, are also required.

In dissecting under the simple microscope, the hands will require some support, which can be obtained from blocks or books at the sides; but the instrument shown on page 40 is provided with hand-rests, as are those of other makers. A portion of vegetable tissue, previously macerated in water, may be simply placed in a few drops of water upon the glass stage. When more fluid is required, watch-glasses may be used, cemented for steadiness into apertures cut in a plate of cork. Or for $1 s$. apiece can be obtained thick plates of glass with deep concave basins ground out and polished, which are very convenient. For some purposes it is needful to use square flat-bottomed glass troughs. It is not always necessary to work under the microscope; with light thrown on the object from the bull's-eye condenser, a great deal can often be done by the naked eye, or with rather shortfocus spectacles (fig. 66).

Many objects, such as an insect, have to be fastened down to be dissected. A plate of cork loaded with lead is often convenient. But it is sometimes better to run into the bottom of the trough some waxy material, into which pins may be thrust. A mixture of paraffin and stearine will be tolerably transparent. Where transparency is not needed, beeswax softened with tallow may be used, and if desired can be easily coloured dark with lampblack. Sometimes an object is best fixed by melting a portion of the ground material with a hot wire, and slightly embedding the object in the melted surface; in other cases, or on cork, pins must be used.

Generally the liquid in the trough will be water. Sometimes diluted alcohol is better, especially in dissecting the 
nervous system of insects, as the alcohol hardens the tissues. Some tissues dissect best in glycerine and water.

Subjects from the plant-world have to be prepared for dissection by long soaking in water to soften them; often that is not enough, and solution of caustic soda of greater or less strength is employed; but this acts very powerfully, and the progress must be carefully watched. Insects to be

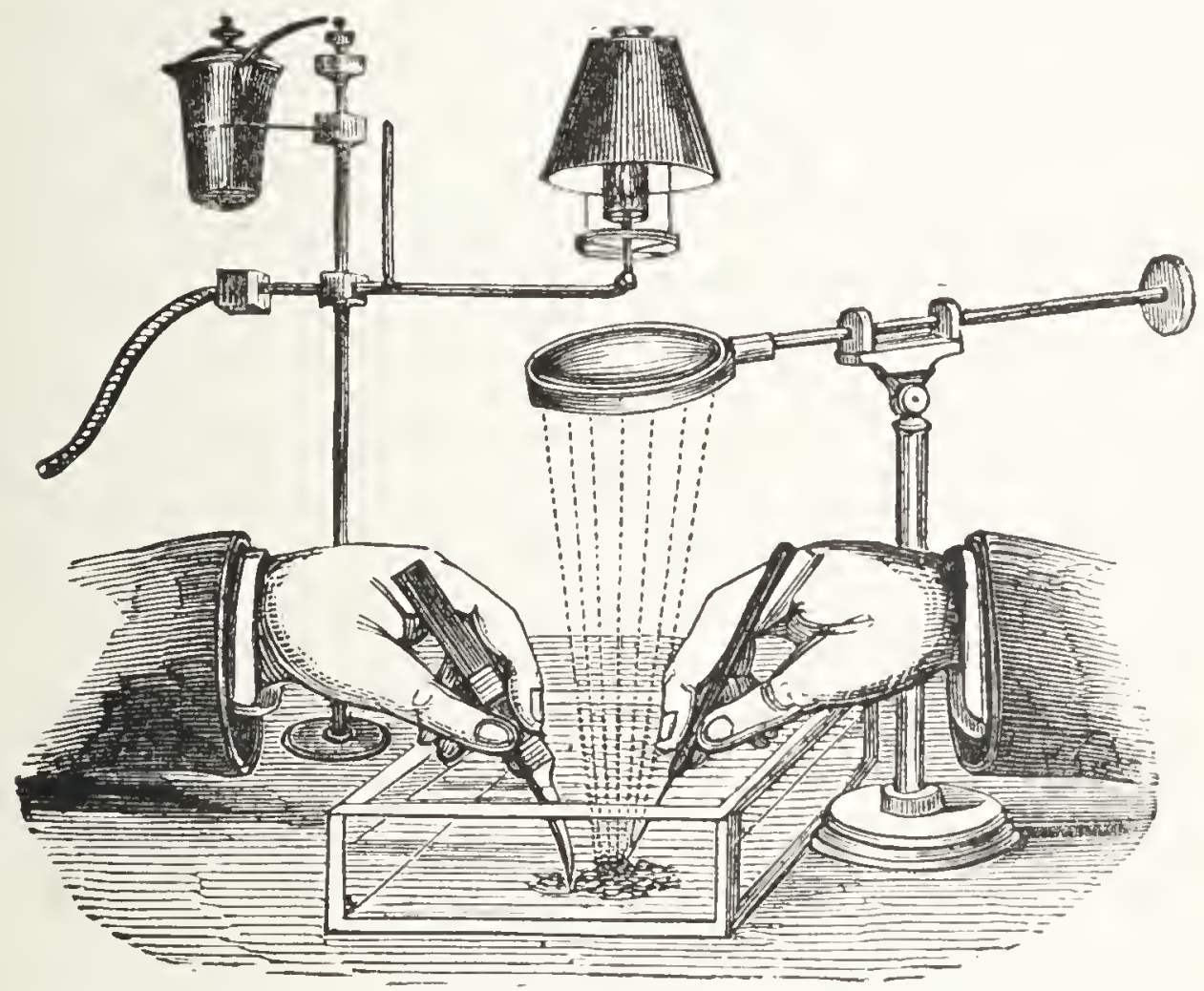

FIG. 66

dissected should always be kept in fluid till wanted; diluted methylated spirit being the most generally suitable.

70. Mounting Slides.-It will best serve the purposes of this work to select from the countless methods of mounting microscopic objects, three only, most suitable for the beginner, and most widely applicable.

Microscopic objects are almost universally mounted now upon glass slips $3 \times 1$ inches. Wooden slips warp, and are discarded even for opaque objects. The paper covers once used have also quite gone out, and the microscopist 
should employ only good flatted glass slips with ground edges. Over the objects are placed thin 'cover-glass,' which can be had either in largish oblong pieces for large objects, or in circles and squares of various sizes.

Before use, all glasses must be cleaned. For very highclass and minute work, strong solutions are often employed to do this; but for the class of objects here in view, the glass will be sufficiently cleaned with a little soap or soda, rinsing in clean water, and drying with glass-cloth, finally polishing with clean linen or a chamois leather. To polish the cover-glasses, after a little experience the finger and thumb may be used with the linen or leather, moving the cover by its edges between them, with finger and thumb of the other hand. Many use two flat pieces of wood with a chamois leather strained over the face of each; the cover-glass is placed on one, the other applied, and the two twisted together. The cover should be reversed, in each case, as it usually sticlis to one of the leathers, and is only rubbed by the other.

71. Cement and Cells.-A very great variety of cements and varnishes, and materials for cell-making, are used by

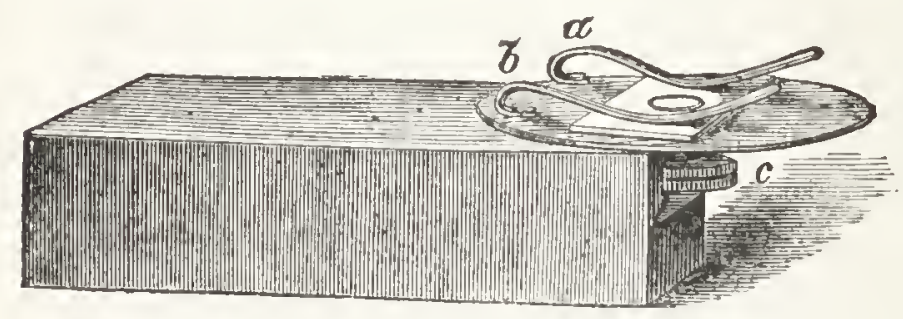

FIG. 67.-Cell-making Instrument (Slialbolt) various microscopists; but for the processes here described a few will suffice. In regard to the walls of cells, very shallow or thin cells are built up with varnish alone. For this process, and for sealing in some cells, some kind of turn-table (fig. 67) is absolutely necessary. That shown is the simplest, and is known as Shadbolt's, the glass slip being simply held down by two strong springs, $a, b$, while the table with the slip on it is turned round by the finger on the milled ring, c. Other forms are chiefly distinguished from this by 
some arrangement or other for automatically centering the slip, so that it shall turn round the centre, and for holding it more tightly. There is, however, no difficulty in centering even with the Shadbolt form. It will be understood in an instant how, if the slip be turned round with the left forefinger, while a camel-hair or sable pencil with some varnish be held touching the slide, a circular ring of the varnish will be laid on. For very minute objects, such as the scales of some insects, one thin ring of varnish lifts the cover-glass sufficiently high; if more be required, this coat is allowed to dry, and a second laid on the top of the first; and even a third or more.

Deeper cells require a solid wall of some kind. Rings of glass, tin, celluloid, and other materials are sold for the purpose. Rings may also be used stamped out with a punch from paper or card, and soaked in melted solid paraffin. Without this precaution damp would enter the cell if dry; or fluid contents might evaporate.

Of cements and varnishes it will suffice to commence with a bottle of 'gold-size,' another of asphalt and caoutchouc in naphtha, called 'black varnish,' and another of somewhat similar varnish known as 'black Japan,' or, what is now preferred by many mounters, the 'Club Black Enamel,' sold for bicycles by the Silico-Enamel Company.

72. Mounting 'Dry' Objects.-By 'dry' preparations are meant such as are only protected from dust by a cover-glass, and not immersed in any fluid medium. Nearly all opaque objects are so mounted, and many transparent ones may be treated the same way, such as scales from insect wings, diatoms, leaves, and petals of plants.

Many of the latter class require only very thin or shallow cells. These are made by 'ringing' a slip on the turn-table with one or more coats of gold size or Club enamel. If more than one be needed, the first one must dry before the second is added; but the final one should only be half dry, or sticky, 
when the cover is applied. Thin flat objects like a piece of wing need no cement, but will have 'spring' enough to keep them in place. Other objects may require a morsel of gum to fix them to the slip, and in all such cases the gum, and the object also if it has contained any moisture, must be most thoroughly dry before covering in, else the moisture will gradually deposit on the cover in small globules invisible to the eye, but too evident when magnified.

Gum cannot be used in this way with very minute objects, such as insect scales or the pollen of flowers. For such, the bottom of the cell may be brushed over with very dilute gum, which is allowed to dry into an invisible transparent film. The objects being placed on the surface, very gentle breathing on the slide, or the steam from some hot water, will moisten the gum again, and make the objects adhere. Or they may be affixed to the underside of the cover in the same way. The gum must be thoronghly dried again before closing the cell. Gum arabic for slide-mounting should have six drops of glycerine per ounce added, to prevent cracking when dry, and a few drops of some essential oil to avoid mildew; the oil of cloves used in balsammounting will answer very well.

The best way to attach scales to the dried gum surface of a slip or cover-glass, is to rub a bit of the wing on the surface. Sufficient will adhere, and can then be fixed as already described. Or, with a hand magnifier or dissecting microscope, a bristle set in a handle may be employed.

When all is thoroughly dried, the cover-glass is pressed down on the 'tacky' surface of the varnish ring. The cover should not reach to the outer edge of the ring of varnish. It should if possible be pressed into contact all round. When the ring appears dry, the edge of the cover is ringed round with one or more coats of black varnish or enamel, or with a coat or two of gold-size first, and black varnish over it when dried. The gold-size and varnish should extend a 
little on the slip, when the varnish cell is very thin, and to the edge of the ring when it is thicker, or when solid cell-walls of any material are used. Such solid cells of paraffined paper or card, tin, glass, or other material, should be cemented on and thoroughly dried before the object is inserted.

Dry opaque objects usually require a cell, and most of them look better on a black ground. This may be obtained with transparent slides by using a 'dark well,' as mentioned on p. 104, but opaque cells are often used. A disk of asphalt varnish may be put on by the turn-table, and the cell built up on that. Or on the black varnish may be gummed a disk of dead black paper, and the cell built up over that. And for some objects it is better to bed a cover-glass on a disk of black varnish, before the latter is dry, and gum the object, or objects (such as a group of Foraminifera) upon the glass; the cell and real covering glass being built up over the whole. The cover-glass is put on and sealed as in the preceding paragraph.

73. Mounting in Benzol Balsam.This is the most durable and generally useful mode of mounting, doing excellent work with entomological subjects, botanical sections, and anatomical sections.

The solution is made by baking Canada balsam on a water-bath, or in a cool oven,'till it is as dry as pitch, and

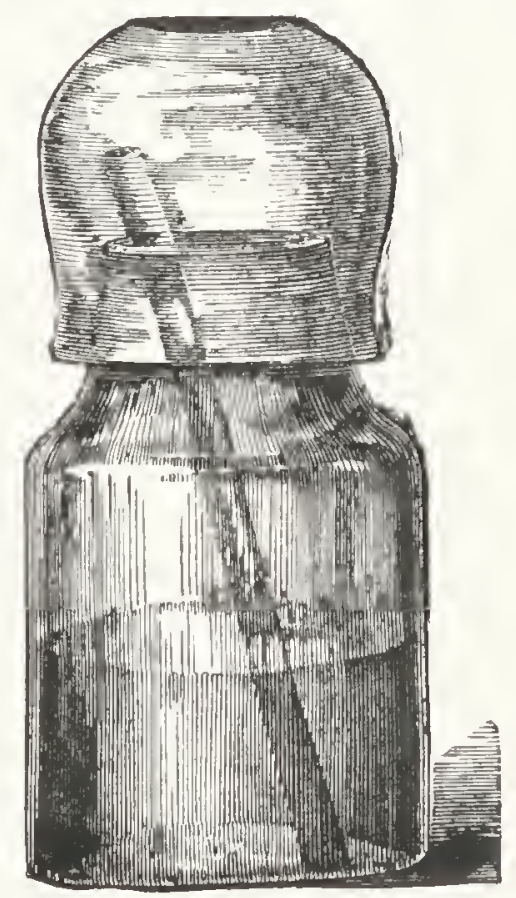

FIG. 68.-Capped Bottle then dissolving it in benzol to about the consistency of thick cream. Usually this requires about equal weight of the dry balsam and the fluid. The dry balsam can be purchased, or the solution ready for use. The latter is to be kept in the wide-mouthed capped bottle, shown in fig. 68, with a glass 
rod drawn to a blunt point, by which the thick fluid can be dropped upon a slide.

Objects are chiefly mounted in balsam without cells, but simply pressed between the slip and cover-glass till dry. If they are too thick for this, as with some hard insects or insect organs, it is usual to employ slips with a concavity ground out of one side. Pressure is maintained either by a small weight, such as a large printer's type, or by one of various patterns of spring clips or compressors, which can be seen at any dealer's shop. Where strong pressure ean be borne, common American wooden clothes-pegs answer very well ; but much less force is generally advisable.

Insects and parts of insects are best kept till mounting in a bottle of methylated spirit. When wanted, an insect is to be soaked in one or two waters for some hours to remove this, then placed in a solution of one part caustic potash to ten parts of water, to soften and dissolve the fatty parts. This may take a few hours, or it may need days, and must be watched; if overdone, the insect is bleached too much to be of use. When the object is thought soft enough, it is well soaked in several waters to remove the potash, and then treated under water with two camel-hair pencils; one in the left hand, holding down the insect by the thorax; the other with a rolling motion, squeezing out the contents of the body, which are carefully washed away. At this stage, if not convenient to proceed at once, the insect may be kept in acetic acid, which, in that case, must be washed out again with water; but otherwise it may at once be placed on a glass slip and arranged, a slip of paper or thin visiting card laid on each side as a wall or protection, and another glass slip being laid over all ; the slips pinching the object are tied tightly together, and put into methylated spirit to get rid of the water; in fact, they should be left in it for hours, and taken out into a saucer of fresh, strong spirit to remove the last traces-really valuable specimens are worth strong 
alcohol. From this the object is removed into spirit of turpentine, and allowed to soak for a variable time. The turpentine gradually makes the brown, hard, chitinous parts more transparent, and some parts may be the better for days of the process, while other organs only need half an hour. It is still better to place an insect from the spirit first into a small saucer of clove oil to remove the alcohol, and from thence into the turpentine; but this is not absolutely necessary. In all cases the turpentine soak is the final stage before transference to the slide.

The mounting may be done in two ways. One way is to place sufficient balsam solution on the slip with the glass rod, take out the object with a tiny spatula, called a sectionlifter, and draining away as much turpentine as possible, work it off the lifter with a needle-point into the balsam if possible, or, if needful, another drop of balsam may be placed on top of the object. The cover-gliss is then taken with forceps and gently lowered on the balsam from one edge, which will prevent air-bubbles. The cover is then pressed down gently, any extra balsam exuded being removed by blotting-paper, a number of small slips of which are cut and kept ready. When all but enough is taken away the slide is put in a clip and laid away in a warm place to harden; or may be gently warmed for some hours on a brass plate, heated by a lamp, but not enough to cause bubbles. ${ }^{1}$

The other way, strongly advised by Mr. Martin Cole, is to put the balsam on a cleaned cover-glass, and get the object well into it; then cover it from dust, ${ }^{2}$ and put away for twelve hours. Warm a clean slip, apply a fresh drop of balsam solution to the centre of the partly dried balsam with the object in it, and lay the cover and object carefully down on the warmed slip, avoiding bubbles. Keep the slide

1 Some mounters prefer to heat the slide over a lamp just enough to causc the benzol to boil very gently, the bubbles escaping at the sides. This is all right if the bubbles do not get entangled in the object.

"A good way is to invert common tumblcrs over slides. 
warmed a few minutes, and press down rather more strongly. The advantage of this plan is that the balsam is more dried, and the object is nearer the cover-glass.

Smaller portions of insects, such as legs, antennæ, \&c., will need scarcely any soaking in potash, or none, but merely enough in turpentine to make them sufficiently transparent.

Portions like wings need only be dried, then soaked in spirit and finally in turpentine, to avoid air-bubbles.

Sections of stems or vegetable structure may be mounted in the same way; their preparation is briefly described further on.

Balsam slides do not need ringing. When dry, all that is exuded may first be cut round the cover and nearly removed with the tip of a penknife, not going quite close. The slip can then be quite cleaned off with a bit of linen soaked with a few drops of sulphuric ether (keeping this well away from any flame). It can be left so, or the slide very slightly warmed again, to gently melt the edges and make them smooth. Should it be preferred, however, a ring of black varnish or enamel can easily be added on the turntable.

74. Glycerine Jelly Mounts.-This method also is most simple for the beginner, and one of the most generally useful to the end. It particularly suits small transparent insects, and vegetable subjects, especially mosses and algæ, preserving the colour well. It can be procured in shilling bottles at any microscope optician's. It is a jelly when cold, but liquefies with a gentle heat.

Objects to be mounted in this have to be finally soaked in vater, or water with a little glycerine mixed in it. Insect subjects therefore only need to be freed from the potash solution; or vegetable tissues to be teased out or dissected in water. The chief thing is to get rid of air-bubbles. It helps this if tiresome subjects are soaked in water that has been well boiled, and finally warmed in it. Insects and 
vegetable subjects which have been 'treated' as before described, are generally already well soaked with fluid.

The jelly is well kept in a capped glass bottle like fig. 68, and must be liquefied by placing in a cup or basin of hot water. Then, a drop or two being placed on the slide, the object upon that, more jelly over that, and finally the coverglass gently squeezed down, the whole should be kept warm till all has well settled down, when the mount may be allowed to cool. Superfluous jelly is cut away with a penknife, and the traces left cleaned with a wet camel-hair pencil.

The slides may be finished and sealed by a couple of rings of gold-size, dried successively, and followed by one of black varnish or enamel. But unless the objects (and consequently the layer of jelly) are thick, they will last for years without any sealing at all. My friend, Mr. Henry Scherren, and the Rev. T. R. R. Stebbing, one of the greatest living authorities upon Amphipods, from whom he learnt the method, are in the habit of mounting their smaller specimens in glycerine jelly with no other preservation whatever; and some of these preparations have reached a quite respectable antiquity.

75. Protection against 0il.-Objects which will ever be examined under oil-immersion lenses must however be protected, whatever the medium they are mounted in. This is done by putting on, outside of anything and cverything else, two successive rings of Hollis's liquid glue, or its equivalent in a solution of shellac in alcohol. This is not acted upon by cedar oil. It is generally best to put on two coats or rings of gold-size before the glue.

76. Section-cutting.-We shall only here describe briefly the preparation of vegetable sections, as of stems, leaves, \&c., which are the most accessible to a novice, and form some of the most pleasing objects. Two implements will be required; viz. a razor, or hollow-ground knife, or plane-iron for cutting, sold for the purpose (though an ordinary razor may often be 
found which will answer); and some sort of a microtome, the simplest form of which is shown in fig. 69. The substance to be sectioned is either embedded or wedged in an inner tube, which slides in an outer one; and is forced up a little for each section by the screw, the knife being kept down to the flat flange surface on the top. Better instruments are sold, with a clamp to fix to the table, a glass flange, \&c.

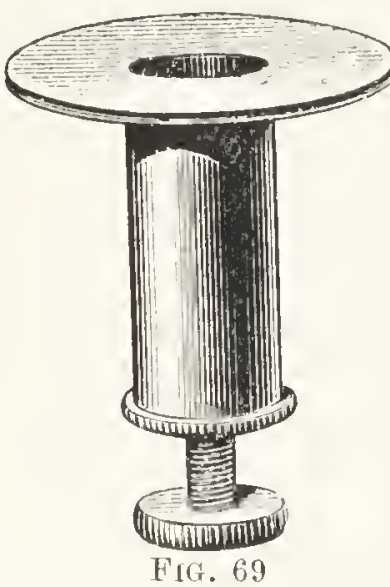

With more complicated apparatus for professional section-cutting this little work has nothing to do.

Wood and stems will need preparation before sectioning. Stems or roots should be soaked some days in alcohol to dissolve but the resins, and then for days in'water to soften, and dissolve out the gurn. Dry or hard wood may need long soaking in water to soften, before the alcohol; or even long boiling may be needed. Leaves and buds, as a rule, only need some soaking in diluted spirit, or may be cut without any soaking at all.

To fix into the microtome, a piece of stem may be wedged in with bits of pith or soft deal; but in most cases it is better to 'embed' objects. Mr. Cole and other's recommend for embedding, four or five parts solid paraffin and one part lard, melted together; but this often slips in the microtome tube, and it is better to use a mixture of stearine and naphthalin, using the less stearine the warmer the weather. This is found to hold tightly. The piece of material should be dried on the surface, and then dipped in a gum solution made as follows:

White Gum Arabic Glycerine

Alcohol (Methylated)

Water

$$
\begin{gathered}
1 \text { dram } \\
5 \text { drops } \\
15 \text { drops } \\
2 \text { ounces }
\end{gathered}
$$

After removal, lay the piece on blotting paper till the surface is again dry. It is then placed in the microtome and 
the naphthalin composition (melted) poured in, the object being held in place till cold.

In cutting, a thick slice or two will first be detached to level down, well wetting the surface, and the razor, with dilute spirit-say ten parts water and one part methylated alcohol. The knife or razor should be dipped into or flooded with the methylated spirit before every cut, and the sections floated off the razor into a saucer of water. This will dissolve off the glycerine and gum, and the naphthalin will float to the surface of the water. Whether embedded or not, all sections are cut with a knife well wetted with dilute methylated spirit, and the razor will need sharpening according to the nature of the material.

77. Staining the Sections.-Most botanical sections require bleaching before further preparation. This can be done in a weak solution of chloride of lime-the clear part of a quarter of an ounce in a pint of water. Some prefer chlorinated soda-the clear part of one ounce of chloride of lime and two ounces washing soda in a pint of water, well shaken after fully dissolving, and left to stand. The bleaching must be watched. When the sections are taken out they should be placed, for an hour or two, in a solution of five drams sulphite of soda in a pint of water to get rid of the chlorine, and then washed and soaked in several clean waters. They are now ready for staining, or may be kept in dilute spirit indefinitely. Two methods of staining will be sufficient.

78. Plain Log-wood Staining.-Dissolve half a dram of hæmatoxylin in $3 \frac{1}{2}$ fluid ounces absolute alcohol, and half a dram ammonia alum in $3 \frac{1}{2}$ ounces distilled water, to which add 3 ounces Price's glycerine and 3 drams glacial acetic acid. Mix the two together and shake, and let it stand in a stoppered bottle in a cool place for some weeks before using. For use add 30 drops of the matured solution to an ounce of distilled water, and immerse the section for a quarter to half an hour, or in some cases more. Then wash well in 
changes of water. Remove the water by a quarter of an hour each in two baths of methylated spirit; clear off the spirit by ten minutes in clove oil, or oil cajeput. Finally mount in benzol balsam. The last operations are the same as in mounting insect slides; but for vegetable sections the clearing oil is necessary.

Eosin is also much used as a single red stain.

79. Double Staining in Red and Green.-This is the most beautiful method of treating stems and many other sections. In no way can the amateur collect such a number and variety of beautiful slides, with little expense and only moderate experience, as by systematic sectioning of the stems of trees and plants. There are many recipes: the following is by Mr. A. C. Cole :-

For the carmine stain, dissolve 10 grains borax in 1 ounce distilled water and add half an ounce of glycerine and half an ounce alcohol. Mix also 20 minims liq. ammoniæ fort. with 30 minims distilled water, and dissolve in this, in a test tube gently warmed, 10 grains pure carmine. When cooled mix the two, and filter into a stoppered bottle.

For the green stain, make a saturated solution of iodine green (also called methyl green) in alcohol.

Stain the washed section in the carmine solution from ten to thirty minutes, then soak in methylated spirit till colour ceases to come out. Wash in clean spirit, to which has been added sufficient of the green solution to make it a bright colour. They may stay in this as long as convenient: then wash in clean spirit, clear this with clove oil, and mount as before.

These stains or their equivalents may be bought ready prepared. Acid aniline green and others are also used. Sometimes it is desired to stain a trial section quickly, and Mr. F. Ritchie states that this can be done in one operation by placing a teaspoonful of ammonia-carmine solution in a watch-glass, and stirring into it a drop of the acid 
aniline green solution sold everywhere for double-staining, taken up on a section-lifter. Dip the section for a few seconds, then remove, and without washing draw off excess of stain with blotting paper. Float and dabble quickly in alcohol, and draw off excess of that; then float in clove oil and mount in the balsam.

Want of space forbids further details, and either of these will produce beautiful results, which cannot be really surpassed. The microscopist who needs further information may obtain it in $\mathrm{Mr}$. Martin Cole's instructions for' sectioning, staining, and mounting in 'Modern Microscopy,' or in Squire's 'Methods and Formulæ.'

80. Charred Sections.-A method of treating vegetable sections mentioned years ago by Dr. Carpenter, is good and easy, and has been too much neglected. It consists in simply charring a section, held between two glasses over a lamp, till it has become charred a dark brown; then mounting plain in benzol balsam.

81. Handling Sections.-What were called section-lifters were formerly much used to transfer delicate sections from the final fluid to the glass slip. They consist simply of narrow slips of thin German silver, with one end bent at a convenient angle. The modern, and in most cases better, practice is to take a clean cover in a pair of forceps, and to introduce this under the section in the last fluid used before mounting. Lifters are, however, often necessary in moving from one medium to another in the processes of washing and clearing, and for balsam mounts.

82. Storage and Selection of Slides. - The most economical, safe, and convenient plan of keeping stides is in the pine boxes of trays, as shown in fig. 70 . These are sold by scores, of two sizes, holding respectively three dozen and six dozen slides, at about $2 s$. and $3 s$. 6d. each. They keep the slides flat and safe at a very small cost, and can be added to just as required. They have the further advan- 
tages, that a selection wanted for any occasion can be looked out and put together; and that, as a collection increases, it can be conveniently sorted out and classified. Double trays holding one dozen are also convenient for a few test slides, or to carry a small assortment to a meeting.

The microscopist is by no means dissuaded from buying slides. Such exquisite work is now produced by skilled. mounters, that much beauty and interest will be missed by the man who neglects to avail himself of it. But he is strongly advised against idly ordering a 'collection.' It is better to pick up a slide every now and then which excites

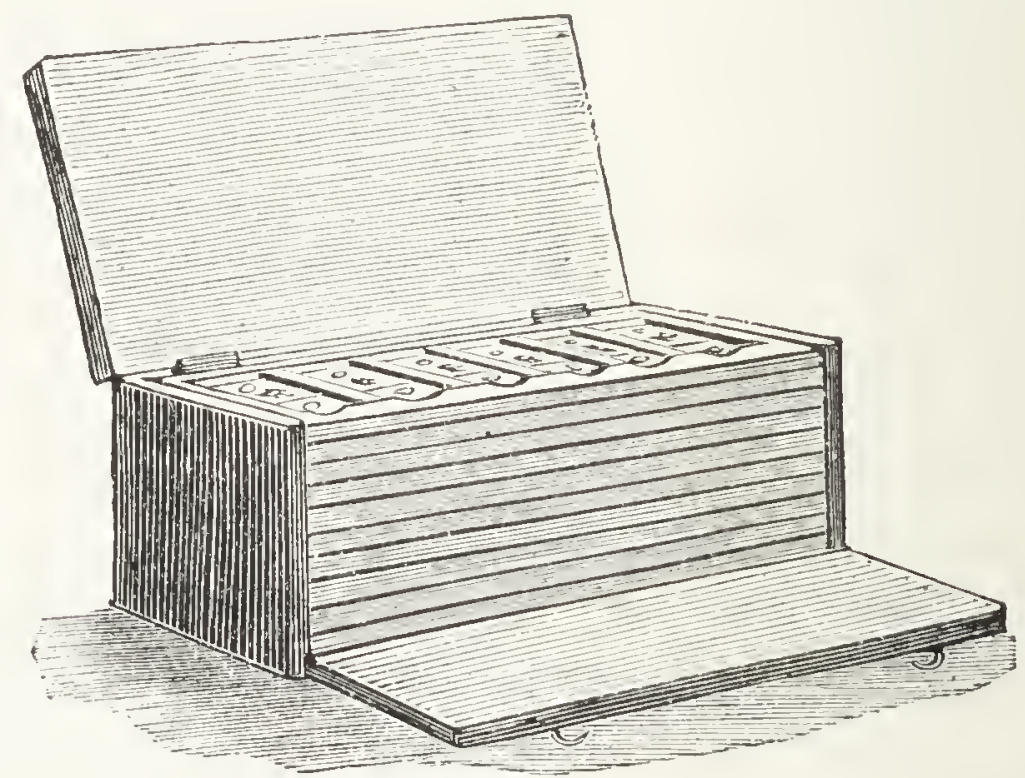

Fic. 70.-Pine case

admiration or arouses interest. Many subjects are advertised from time to time. Bought in this occasional way, a little expenditure gives much more enjoyment, is much less felt, and keeps up the interest, which every purchase will renew as it is made. Slides will also be only purchased when a really good one offers; and a much higher average of quality will be secured. The few hundreds of slides which I possess, each chosen with a view to illustrating some occasional lecture, upon the screen, with the lantern microscope, have all been selected in this way, better 
specimens of some objects being substituted whenever they offered. Thus the very collection becomes a source of interest and gratification, whilst the cost is spread orer many years.

\section{CHAPTER VIII}

POND AND MARINE LIFE

The space remaining in this little volume for some rough outline of what the microscope has to reveal, is quite insufficient for any strict orderly classification. We must divide this part of our subject into a few large and very roughly comprehensive groups, and attempt only a brief survey of each, with some practical hints from a general point of view. Thus the chapter following this, though entitled 'The Insect World,' is made to include spiders, which are only insects in common parlance, and not in the scientific sense at all.

We allot purposely more proportionate space to this chapter, brief as it must be, than to the remainder, for special reasons. It is rather desirable, after the novelty of the instrument, and the amusement and interest of such slides as were borrowed or purchased with it have worn off, that the budding microscopist should commence real work for himself with this branch of study. It is in itself one of the most interesting branches - for there is always a living interest about life and motion. It carries the student into country air and life, and affords him a dash of that 'sporting' interest which has such an attraction for the average man. It gives him something actually to do, out of doors as well as indoors. It teaches him from the first to look for objects of interest and study, and to look below the obvious surface of things for them. And it often leads to pleasant acquaintance and 
companionship, especially if a good society of working microscopists be within reach. ${ }^{1}$

83. Collection of Pond Life.-A few glass tubes with corks will be required, an inch wide by three or four inches long being a good size. Half-a-dozen will bring home enough for some little time, and larger bottles or jars are only required when it is meant to stock an aquarium. And there must be some kind of 'collecting-stick,' with appliances fitting into the end. It is to be feared that a well-known and fascinating book ${ }^{2}$ upon this subject, is responsible for a great deal of disappointment and useless work. I myself certainly gathered from it an impression that the interesting subjects described were, as a rule, obtained by dipping into ponds a small wide-mouthed bottle at the end of a stick. Such is of use occasionally, chiefly to introduce (upsidedown and empty) into some special spot, in order that when inverted it may collect a specimen of what there may be just there and nowhere else; but a novice no better equipped and instructed than that, would have long and tedious work for very little return. The really useful implements are shown in figs. 71, 72, and 73 .

The chief of them all is the net, shown in fig. 71. This is funnel-shaped, and attached at the wide end to a ring 6 inches diameter, while the narrow bottom end is clasped by a rubber ring round a flanged glass tube an inch diameter and three or four inches long. The net is made of rather fine or mull muslin. As this net is swept through the water, either on the surface or elsewhere, the water passes through it, but the rotifers, crustaceans, small larvæ, or whatever else

1 The London student cannos do better than join the Quekett Microscopical Club, whose frequent organised Saturday excursions each season will find him companions, and initiate him into practical pond-hunting under the guidance of skilled old hands. Once at least every season there is also a marine excursion. The subscription is only ten shillings per annum, which includes the Journal. There are many local societies with similar pursuits in different parts of the country, which can be found on inquiry.

2 Marvels of Pond Life. 
was in the water, are retained; then finally when the ring is turned into a horizontal position and the whole lifted out of the pond, most of the water drains away, and nearly all the contents are condensed into the flanged tube. This need not be removed from the net; but by bringing up the mouth above the ring, the contents can be poured out into a bottle or another tube. Where a mere bottle would bring up hardly anything, a few sweeps of the net (supposing of course that material is really present) will produce a tube crowded with specimens.

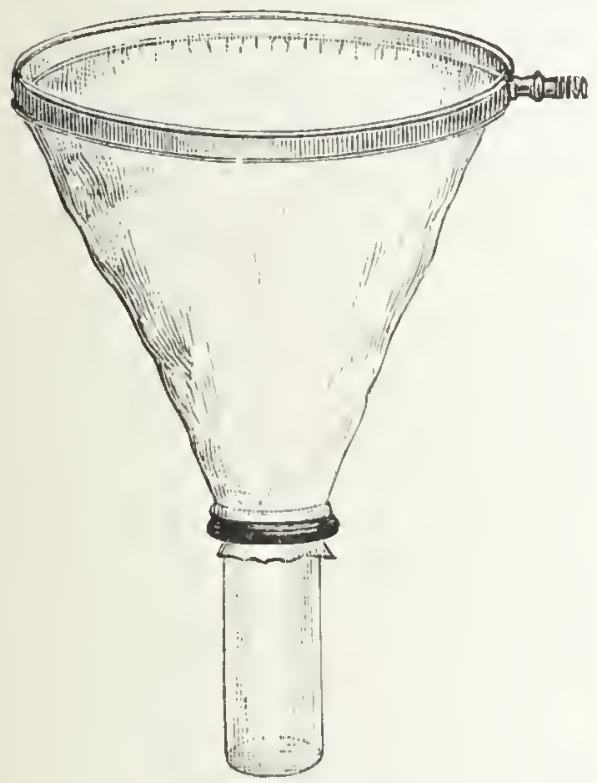

FIG. 71.-Ring, Net, and Tube

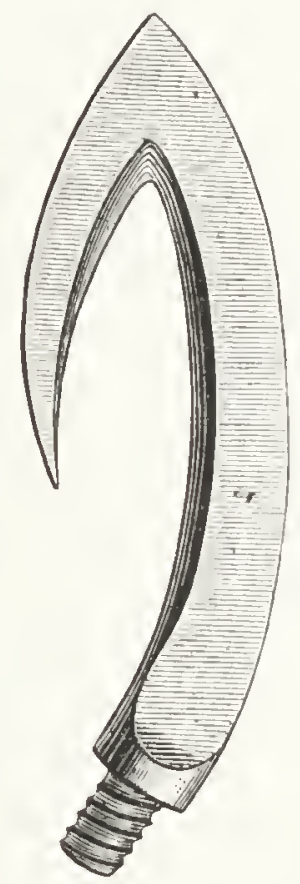

Fia. 72.-Hooked Knife

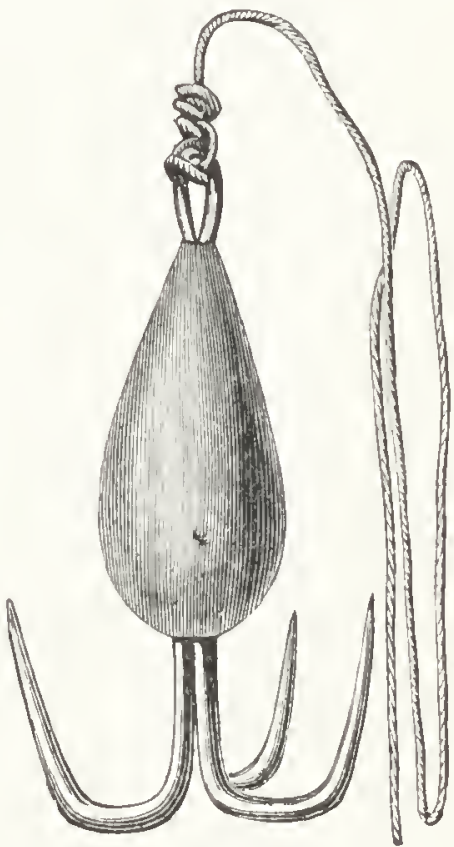

FIG. 73.-Drag

Of less importance, but useful occasionally, is the hooked knife in fig. 72 , by which portions of plants may be both severed, and drawn to land. In such cases the rushes or other plants should be cut as low as possible; when polyzoa may often be found that would be searched for in vain near the surface.

A proper collecting-stick, fitted, costs from $12 \mathrm{~s}$. to $15 \mathrm{~s}$. and is made telescope fashion, in three lengths, resembling a walking-stick when shut up, and stretching almost three times the length when extended. It usually includes a 
small ring for a bottle, which is however seldom of use. A good stick is well worth its cost; but when every shilling must be studied, a little ingenuity can fit a ring of wire to the end of any ordinary stick or pole, to carry the net. And it is the net and tube which is the essential implement.

Another useful apparatus is the drag hook (fig. 73). This also can be made by anybody, casting two or three ounces of melted lead round the hooks, fashioned out of wire. There is no use in having the points too sharp, and some danger. Three hooks equi-distant are best. This will answer most purposes of the knife, as it can be thrown into a mass of weeds, and will drag out a portion as well as the latter. It will also drag a plant up by the roots. And it is also very useful in dragging up sticks or branches or dead plants lying on the bottom, to which may be found attached many forms of life that cannot be found near the surface.

A few hints must suffice for what may be found, and how and where it should be looked for." Infusoria, freeswimming Rotifers, Desmids, Volvox, Water-fleas and other crustaceans, and miscellaneous small fry, will be obtained by sweeping with the net, examining the result of every cast with a pocket-magnifier, in one of the tubes, unless it is seen at once what has been obtained. A pond cannot be found that will not yield something; but it may not be what is wanted. It will soon be found also, that the kind of pond affects what is found. A different run of Rotifers will come from a pond in a farm-yard polluted by manurewater, and from a clear one; and what may be called 'clay-muddy' water will also have character of its own. Water-fleas and crustaceans like sun and open ponds: many rotifers and infusorians the same : others are found in

1 For fuller hints and details respecting the contents of this chapter, the reader should consult Ponds and Rock Pools, by my friend Mr. Henry Scherren, F.Z.S., to be obtained of the same publishers as this book, and to which I am indebted for soveral of the illustrations. 
deep shade, or under pond-weed. Volvox and Desmids also like open water and sun; and most of the algæ are found in the same circumstances.

The stationary and tube-dwelling rotifers must be sought differently, on the pond-plants. The common weed Anacharis is one of the greatest favourites of these. Portions may be dragged to land by the hook or knife; but often it 'pays' better to reach spray after spray from the bank, and examine by the lens in one of the tubes, throwing back each bit if it does not bear anything interesting. It requires a little attention and practice to recognise Stephanoceros or a Floscule, even with a good glass; but Hydra, Melicerta, and Stentors are easily seen. The favourite localities of Vorticella are the rootlets of duckweed, and the general appearance of a group of Vorticella or Carchesium is that of a tiny lump of bluish-white mould or scum. Any such morsel of apparent 'mould,' upon almost any water-plant, will repay examination, unless the mi-. croscopist already knows what it is.

Polyzoa are more rare, and must be sought for more persistently. As a rule they are found more or less below the surface of the water, preferring shade. Rootlets of trees on the banks of ponds and streams, a foot to three or four feet deep, or submerged stems of plants, or submerged sticks or branches, are the usual places. These have been so 'hunted' by collectors and Clubs (at least in the neighbourhood of London, and doubtless elsewhere) that they are more difficult to find than formerly. Trips in the country should be utilised for them as opportunity offers. The first time I ever found a really fine lot of Alcyonella was upon a tricycle excursion, a few miles from Herne Bay; on the rootlets of pollard willows, two feet beneath the surface of a perfectly clear stream, were large masses. Let the collector rigidly obey the golden rule, and whenever he finds a prize, take no more than cnough for his needs, leaving the 
rest for others, or to multiply in future. I have known a so-called 'microscopist' nearly strip a pond in Epping Forest of the beautiful polyzoon just named (Alcyonella), carrying home a number of large bottles, the great bulk of which were of no possible use to him, but would speedily become a mass of decomposition. There is some reason to fear that rare Polyzoa may be hunted to extinction in a populated island like ours, as much as giraffes and elephants in Africa; and such destructiveness cannot be too much condemned.

Many infusoria and rotifers are best collected with the small bottle amongst the stems of rushes or other waterplants. Others are found chiefly amongst the moss or confervae that fringe walls or boards at the sides of canals. Diatoms and desmids are found in the green and brown films which cover the mud at the bottom in many places. Amœba, Actinophrys, and other lowly forms of life are to be sought for amongst the flocculent mud or cébris which falls to the bottom of the tubes, or of the micro-aquarium.

To what was said on handling objects in $\$ 68$, it only need be added here, that it is often well to look over a good spray of some water-plant in a comparatively large trough, with a low power such as $1 \frac{1}{2}$ inch or 2 inches. A great variety of objects will often be found, which can then be separated. For detailed examination it is generally best to put into a trough (as thin as possible, and filled with clean water) a single leaf or small bit, with the specimen attached, that the view may not be obstructed. Such small bits of plant with animals attached, are easily handled with a slender pair of forceps.

84. Water Fleas and Entomostraca.-Taking first in our broad view what is likely to come first to hand, the most evident result from casts of the net will probably be a catch of these, which belong to the larger group of Crustaceans, containing crabs and lobsters. The word Entomostraca de- 
notes an 'insect in a shell,' and will be seen to be very appropriate, though it is a curious example of an entirely 'popular' meaning rendered into Greek. The proper' waterfleas or Daptunice well deserve their name, from their size, general look, and peculiar jerky motion in the water. There

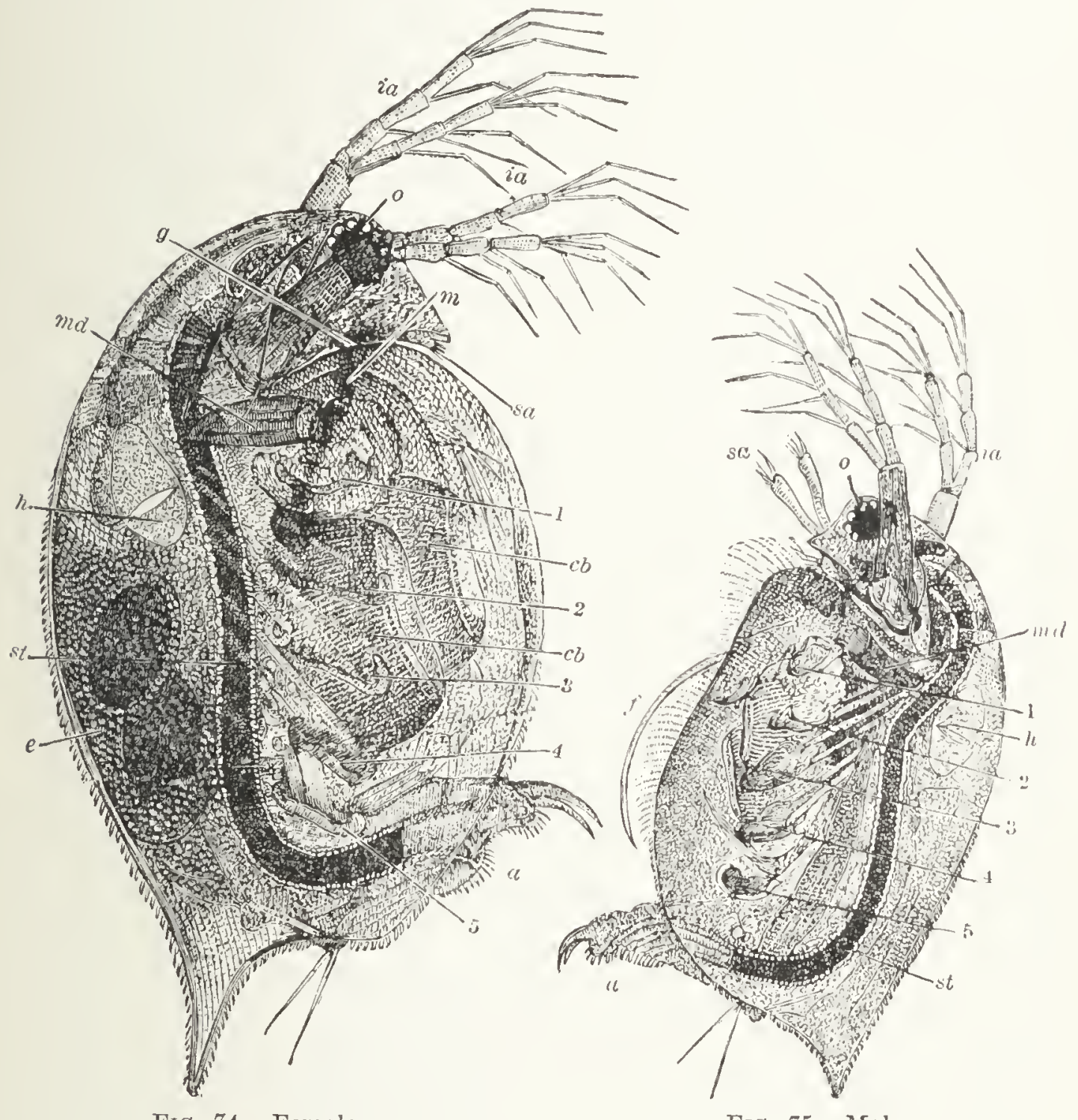

Fig. 74.-Female

FIG. 75.-Male

are seven or eight species, which differ in size and shape, but have a strong general resemblance; and they are very common in open ponds which are not very constantly disturbed. So numerous are they sometimes, that clouds of them may be seen in the water in the summer; either 
whitish clouds like oatmeal, or in other cases of a quite reddish colour. One dip of the net into such a cloud will catch hundreds, sometimes of two or three species; but in almost any pond not much fouled, the net will soon collect some. They are interesting objects, because of their transparency, which allows the digestion, the heart, and blood circulation to be easily seen; and they are easily examined by pushing into a trough just narrow enough to squeeze the enclosing shell slightly together, so as to check their motion; or by very slightly squeezing in the live-box.

Fig. 74 represents the female, which is much the largest, and fig. 75 the male, of Daphnia psittaca, and fig. 76 is a rough diagrammatic section across the

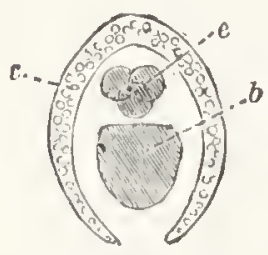

FIG. 76 body. The shell is not hinged, but joined in one as shown in fig. 76, though it opens and closes to a considerable extent by elasticity at the back. Its outer wall is hard and reticulated, the inner soft, and in the space between globules of blood ( $c$, fig. 76) can be seen in motion. In most varieties the shell ends in a pointed spine, and the edges are often serrated or bordered with teeth.

The head culone of the body is outside the shell. It ends in a beak, which is, however, not a mouth, and its most conspicuous appendages are a pair of superior antennae $s a$ (sec figs. 74, 75) which are near the beak, and very small in the female, and a much. larger pair $i a$ of inferior antennae, which spring near the neck. These last divide into branches, which are furnished with filaments thickly studded with finest hairs. With these large antennae the creature mores or swims, so that they must be considered a pair of fore limbs.

There is but one eye o, though its growth has been traced from two spots, one on each side. It has twenty or more lenses on a black spherical surface, and is shown more clearly at $o$, fig. 77 . 
Farther back come the real mouth $m$ and the mandibles $m d$, shown more clearly also in the front view of the head of $D$. vetula, fig. 77. They work from the sides of the head as in insects, and the serrated ends grind together. The D. vetula here figured, opens the sides of the shell more than usual, and can often be got on its back so as to show this front view; it is pretty common, and may be known by its nearly round profile and the absence of any spine at the end, as shown in the side view of it, fig. 78. Following the mandibles come a pair of serrated maxillae or jaws, much smaller.

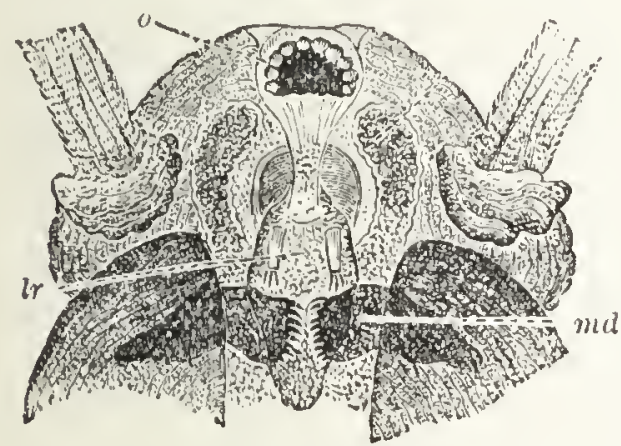

FIG. 77

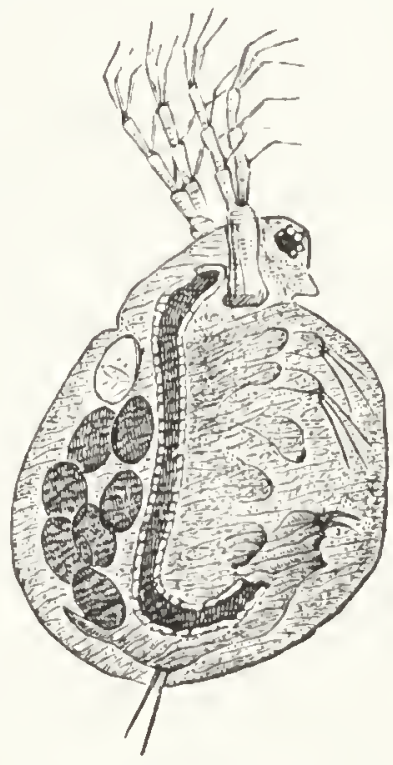

FIG. 78,-D. vetula

The next appendages are interesting, and consist of five pairs of 'legs,' numbered 1, 2, 3, 4, 5, in figs. 74, 75. There is no doubt that they answer to legs in other crustaceans; but the wonderful variety of result, and adaptation of means to ends, of which the Creator's ' natural laws' are capable, is shown in the fact, that whereas in these creatures the antennae have become the locomotive organs, the function of the legs is to assist in feeding and respiration! The first pair may be excepted perhaps from these functions, offering (as seen in fig. 79) the most conspicuous marks of sexual distinction, next to the presence or absence of eggs; for while the first pair in the female have branches or appendages, those of the male are terminated by a hook and 
single curved filament, which projects beyond the shell as at $f$, fig. 75. The next three pairs are furnished with peculiar comb-like structures (cb, fig. 74) (more especially developed in two of the pairs) and shown magnified to a much greater degree in fig. 80, termed the branchial plates. These are in constant motion, and serve to create a current of water up between the sides of the shell. This both conveys infusoria and other particles of food to the mandibles, and air to the gills or breathing organs. At one time it was thought they

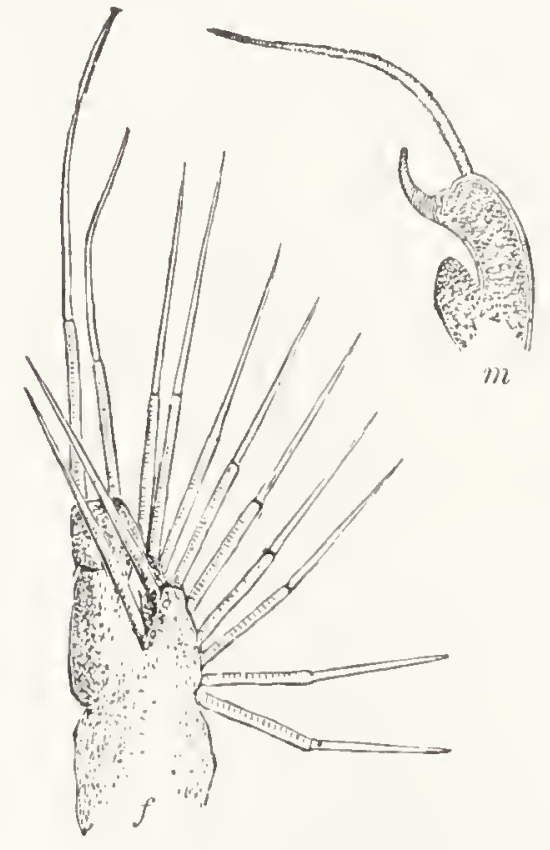

FIG. 79.-Forelegs $f$. Female $m$. Nale

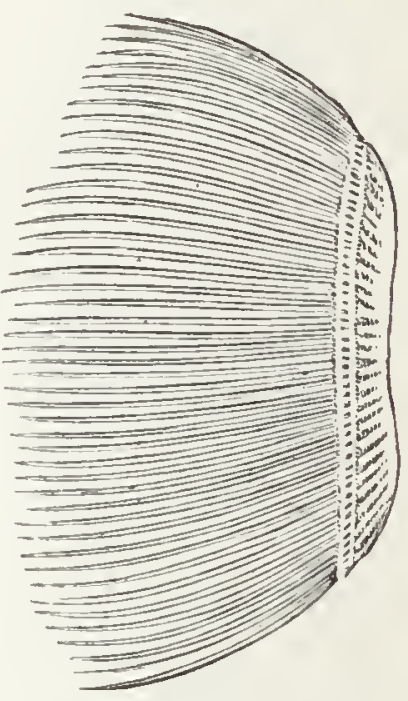

FIG. 80.-Branchial plate

were gills; but the real respiratory structures appear to be certain pouches also attached to the legs, and seen most clearly in fig. 75, in whose walls the blood can with care be seen to circulate rapidly. The hooked 'tail' of the animal, curled round to the front, and often protruded considerably from the shell, is constantly passed up and down between the pairs of legs as if to cleanse them.

The stomach and intestine st are in one, and the contents can easily be seen in motion, until the refuse matter is ejected at the anus $a$. The heart $h$ is a structure with a 
kind of cleft in the middle, which opens and closes in beating, and the whole will be seen to pulsate vigorously.

Passing over many other points of detail, we can only refer in conclusion to the wonderful provision made for the propagation of the species. The body of the animal is only united to the shell about as far down as the heart, and beyond that is quite detached. The eggs are first formed in ovaries at the sides of the stomach-intestine; but are soon transferred into the space between the back of the animal $b$ and the shell, as shown at $e$ in the diagrams, figs. 76, 81. Here, in the ordinary course, they gradually mature until ready for 'hatching' about the fifth or sixth day, when the young emerge into the water. Their progress can be easily seen in the microscope. This is the ordinary mode of propagation; but it is believed that all such eggs, and probably all the adults, perish in a cold winter, and this has to be provided against. Like larger crustaceans, the Daphnia has at intervals to 'moult' its shell for a larger one. At certain intervals, a thickening of the shell takes place

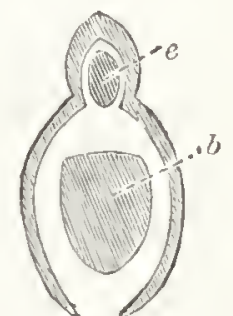

FIG. 81 previous to this, at the back, and an extra chamber (e, figs. 74 and 81 ) begins to form outside the ordinary hatching cavity. At first this opens out of the latter, as in fig. 81 ; but the cavity gradually closes entirely in, with from one to three eggs $e$ within it-generally two, forming a sort of saddle (fig. 74,e) which is hence called the ephippinum. The eggs so enclosed, also acquire much more thick and horny shells than the others, and do not hatch at the same time. Next moult sees the ephippium thrown off; to float about probably until the spring, when the eggs thus doubly-protected hatch out in the rays of the sun.

Another of the Entomostraca, known as Cyclops (fig. 82) is more common even than the Daphnie, being often found in drinking water. Scarcely any piece of water will fail to yield specimens of this, but it does not abound in such 
multitudes as the preceding. In this creature may be noted the single eye (whence its name) and in most specimens (the great majority being females) the

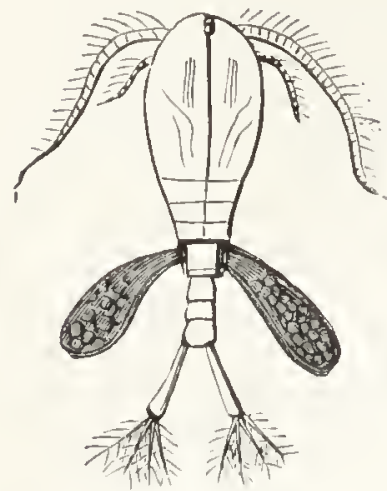

FIG. 82.-Cyclops curious pair of bags of eggs dragged about by the parent till ready to hatch; also the curious resemblance in form to the larger Crustaceans. Except to the systematic student, however, there is not so much interesting detail as in the Daphniæ. There is another small crustacean pretty common, the Cypris genus, which may be known from its oval bi-valve shells.

85. Infusoria.-Next to Entomostraca, and such larvæ and other larger creatures as are easily weeded out, the most plentiful product of collection will probably be a

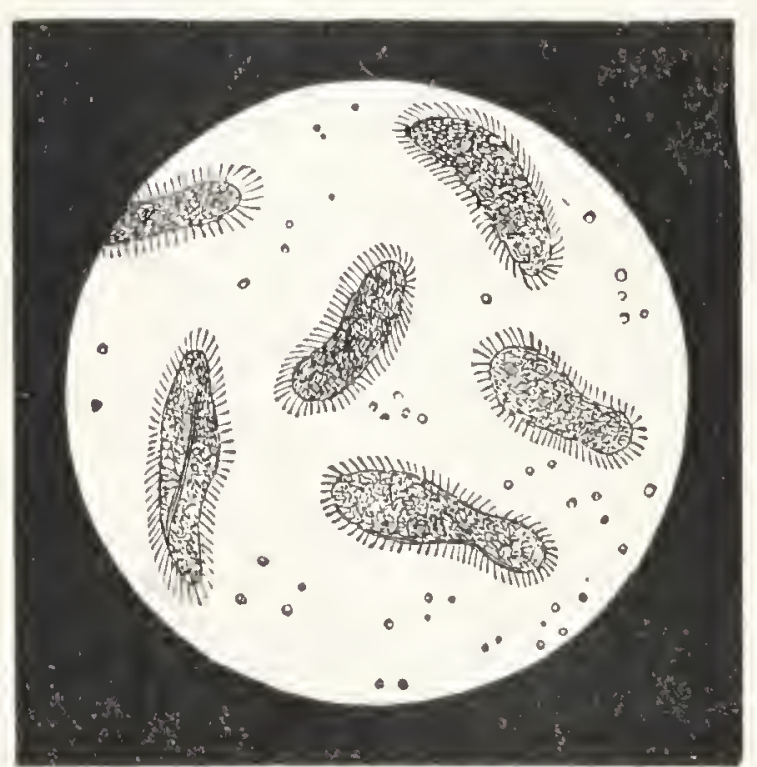

FIG. 83.-Infusoria number of really microscopical animalcules, or Infusoria; so called because first observed in infusions of vegetable matter. Indeed, as good a way as any of procuring many forms, is to place a little hay in a tumbler of water and leave it awhile; or many of the monad forms breed most freely (from the infinitesimal spores which are constantly floating about in the air) in water containing animal matter, such as bits of meat, or of the head of a codfish. Taking our pond-water, however, and putting a drop on a slide, (only a cover-glass placed over the drop being needed for these small creatures) the field of the microscope will be seen more or less occupied by tiny bodies in motion. Some may be Rotifers, to be mentioned presently: but smaller 
than these may appear as in fig. 83. The small dots we must leave for advanced microscopists. One of the larger creatures is Paramecium, and it is easily made out that the body contains near one side two contractile vesicles; that there is also a nucleus, and one or more vacuoles or spaces for food; that there is an opening on one side, evidently a mouth; and that the whole body is covered with fine hairs, known as cilia, in constant motion, by which the creature swims, and by which food particles are swept into the mouth. These particles enter the vacuoles, which after awhile approach the surface and reject the refuse.

Nearly as common is the Swan animalcule (fig. 84), named for obvious reasons. Very different from the other,

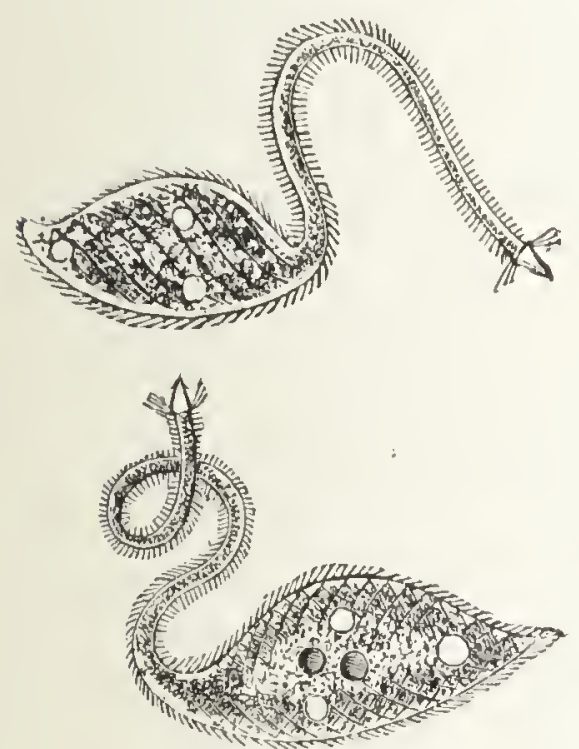

FIG. 84.-Swan Animalcule and twisting about in the most ludicrous way, the same general features may still be observed. So also they may in the Barrel

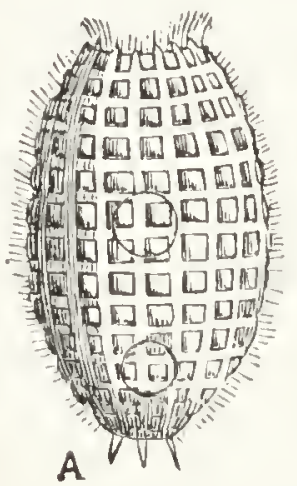

Fig. 85.-Barrel Animalcule A. Adult B. Dividing

animalcule (Coleps hirtus) shown in fig. 85, which in general appearance is more different still, being sculptured into rectangular areas on the outside. This is also very common, and uncommonly voracious. We have purposely here selected three of both the most plentiful, and apparently most diverse.

The Ciliated Infusoria are of strange significance in the vast scheme of animal life, especially as regards the 
cilia from which their family name is taken. These are evidently sense organs and motor organs, and especially cause motion in surrounding particles. On the one hand, it is deeply interesting to trace back their possible beginning in still lowlier forms; and on the other to see (as we shall in Chap. XI) how ciliated cells have been preserved in the highest animals.

These creatures are also of unique interest as being, according to the majority of biologists, those in which 'the life of the single cell finds its highest expression.' ' Very much materialistic argument has been based by a certain school of biologists upon 'the single cell,' and upon the utter 'absence of structure' in the protoplasm ( $\$ 86)$ of which it is said to be composed. Any one of these 'single cells' is simply crowded with 'structure' and with marvels. It has a skin, outside which the cilia protrude; and this skin consists of distinct and different layers, one of which is strangely striated, much like muscle. And while the mode of multiplication for a while is that of gradual constriction in the middle (as seen in the Coleps, fig. 85) and ultimate transverse division into two; at intervals two individuals conjugate, to form as the result, and often in definite parts, ova ${ }^{2}$ or spores. The higher power we put upon it, the more the 'single cell' teems with details clearly differentiated, which only the imperfection of our lenses hid from us till a few year's ago.

86. Lower and Higher Forms of Single-celled Animals. Leaving this question for the moment, let us take a look at still lower forms of our ' single cell.' Lower than the ciliated, are the flagellated Infusoria, which only possess one, two, or three whip-like filaments, by the motion of which they swim. These flagella are so inconceivably fine, that in many cases only the finest objectives and most skilled manipulation

1 Dr. Dallinger, summing up biological opinion,

2 Balbiani and Griiber. 
will reveal them; and we must simply state, that here again more perfect lenses, following twonty years' almost constant obscrvation, have only lately revealed most marvellous and complex structure in what was formerly called 'structureless.' Especially in that smaller 'nucleus' which was once held to be simple and homogeneous, it is now demonstrated that wonderful changes of structure invariably precede and originate the more obvious changes in the outer portion of the animal. Only with the most modern lenses were these facts possible to be observed.'

We may go to lower forms still, and look at the Amcba, the Materialist's special favourite. It is apparently a formless lump of jelly, varying from $\frac{1}{100}$ th to $\frac{1}{40}$ th of an inch in diameter, and plentiful everywhere, but troublesome for the beginner to find. It is generally found amongst the flocculent sediment at the

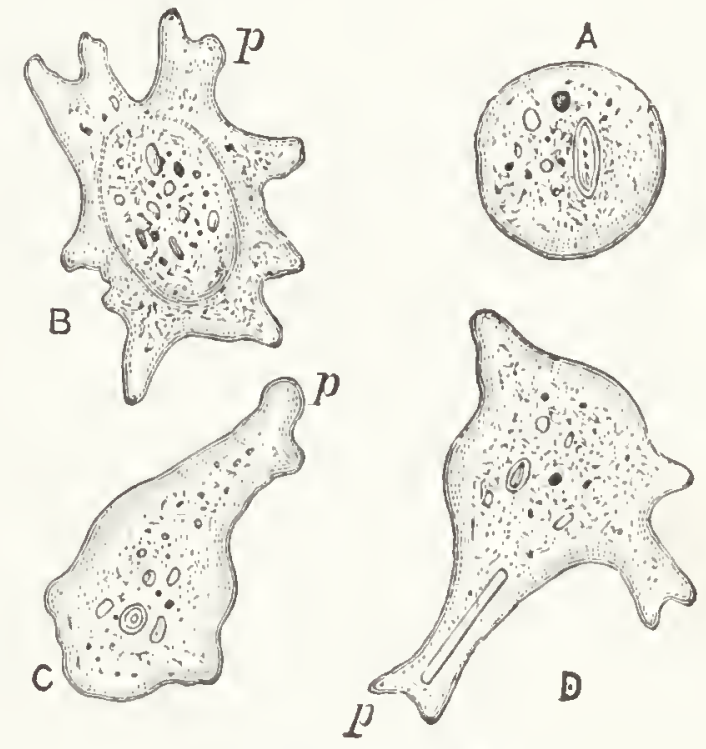

FIG. 86.-Amœba bottom of tubes, taken from ponds of water near decayed vegetation; and is seen as a little shapeless spot of transparent matter moving slowly across the field of the microscope, pushing out projections now here and now there, and contracting somewhere else. Some are clear, others orange-coloured. In the indefinite extensions of this lowly form of life, whose normal state may be taken as at $\mathrm{A}$ in fig. 86, while extensions are shown at $p$ in $\mathrm{B}$ and $\mathrm{c}$, we have probably the first approach to flagella and cilia. There is here no definite vacuole or stomach, but these pseudopodia, as they are called, reach out to and engulf and flow round some particle of food, as at $p$ in D of fig. 86. Even here, ${ }^{2}$ Dr, Dallinger's Presidential Address to the R.M.S. 1886. 
however, is seen the nucleus, and the cavity which alternately expands and contracts, called the contractile vesicle, and a distinctly firmer skin or ectoderm, and a number of very fine granules which can be seen flowing about.

There are other low forms of life, which Professor Hæckel especially insisted upon as consisting of 'an entirely homogeneors and structureless substance, a living particle of albumen,' or again, as that eminent Materialist expresses it, ' the whole body of the Monera, however strange this may sound, represents nothing more than a single, thoroughly homogeneous particle of albumen in a firmly adhesive condition... Our sharpest discrimination can detect no trace of an internal structure.' We were taught that in a mere 'homogeneous' particle of matter, the result of chemical combination, resided as a 'function' all the essential characteristics of life. This was, and is still in some quarters, stated as the now ascertained result of modern scientific knowledge. ${ }^{1}$

And all this is now known to be simply the result of ignorance and imperfect observation. It is the microscope, in its highest forms, which has made the truth known. As Dr. Dallinger records, (Quekett Club, Feb. 20, 1891) 'it was gradually shown that a distinct structure was discoverable in some cells, and subsequently it was shown that nearly all cells and all forms of protoplasm, show a microscopic network of fine fibres. In short, it became plain that the reputed structurelessness of the cell was due to the inefficiency of the lenses used, and was dissipated when competent optical aids were employed.' Down to the very bottom of the ladder a most delicate and complicated 'structure' is found, whose further exploration still baffles our utmost efforts at

1 See various passages in The Story of the Creation, by Edward Clodd. And Mr. Herbert Spencer, from whose Principles of Biology much of it is taken, writes himself that 'the lowest living beings are not, properly speaking, organisms at all, for they have no distinction of parts, no traces of organisation,' 
decipherment; but the more power the microscope can bring to bear, the farther into the background recedes that inscrutable mystery of Life, which the sheer ignorance of men had rashly claimed to have unveiled.

Let us now take the upward direction. In the so-called Sun-Animalcules (Actinophrys and Actinospherinum) which can with a little search be always found before long, we have a nearly spherical central morsel of a cell, with many thin filaments projecting radially in all directions-not nearly so thickly as the cilia we began with, and longer, but obviously pointing in that direction. It is to be specially noted that many other small animals planned on this type have the power of forming curious stclls round themselves, of either calcareous or silicious matter, which are pierced with tiny holes through which the pseudo-

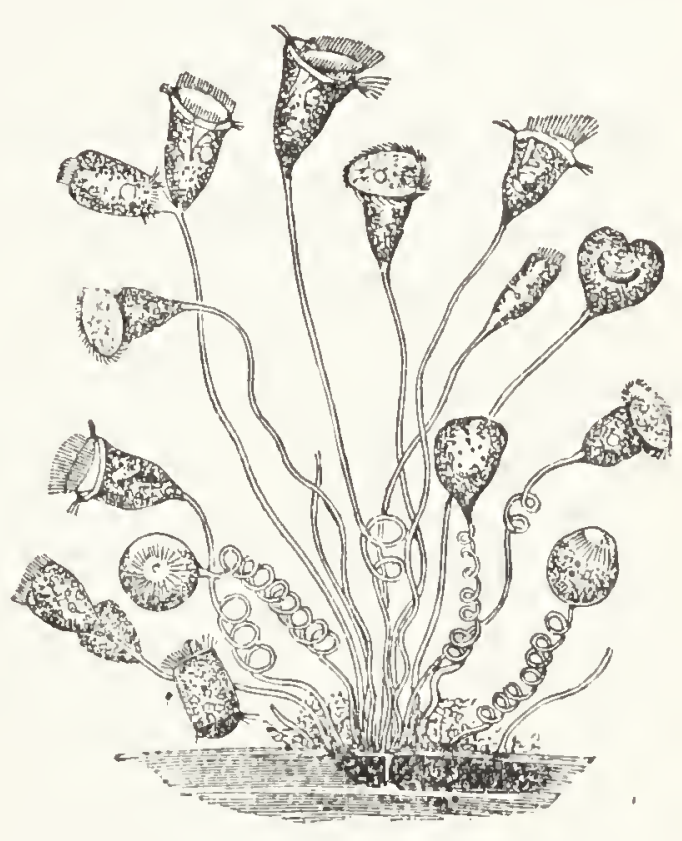

FIG. 87.--Vorticella podia protrude. These creatures are then the Foraminifora, or the Polycystina and other Radiolaria mentioned in Chapter XII.

Passing by the forms of ciliated Infusoria from which we started, we shall arrive at higher types of the supposed 'onecelled' animals. One of these is a plentiful and lovely microscopic object. By examining in tubes a few little portions of duckweed from almost any pond, there will generally be soon found what looks like a little white mould. Any stalk of this or other vegetation with such appearance, put into a trough and examined, will probably appear as the Vorticella, or Bell-Animalcule (fig. 87), the cell assuming a vase-shaped form. The rim is not quite circular, but of a 
kind of spiral shape, with a sort of disc inside; and it is round this rim cilia are developed. Their motion takes place in such successive order as to produce the idea of circular motion; and it does produce an actual vortex in the water, by which particles of food are swept into the cup or mouth, to enter vacuoles and be digested as in the other Ciliata. The contractile vesicle, and other parts of the so-called 'cell 'will also be observed. The bells are as a rule attached to long stalks, which either stretch out to great proportionate length, or every now and then contract into a short spiral: this motion takes place independently in each stalk, so that the whole group is in constant motion in a beautiful manner. The most striking way of exhibiting Vorticella is upon the dark ground, with the stopped condenser, paraboloid, or spot-lens (\$56).

Reproduction takes place by division, both across, and down the middle, as in other Infusoria. But as in their case, at intervals there has to be a conjugation or pairing of two individuals, resulting in the production of swarms of spores. The cells are also capable of a quite free, swimming existence, apart from the stalks. These stalks reveal a striated character, with a strange resemblance to that of contractile muscle in the higher animals.

The stalks of a colony of Vorticella usually spring at the base from a little gelatinous mass, thus showing some approach towards a colony or assemblage of many animals. This kind of development finds fuller expression in actual tree-like combinations, known as Epistylis, Carchesium, and Zoothamnium, in which the stems actually do branch off from larger stems, with differences which need not be described here. ${ }^{1}$

The largest animals of this type are the Stentors or Trumpet-Animalcules, of which the finest variety is known as Stentor polymorphus (fig. 88). This is as much as $\frac{1}{20}$ th 
of an inch long, and generally green in colour: in one splendid variety, here figured (it is not very common, but in no other respect departs from the type), the green is studded with beautiful scarlet spots. The disc and rim by the cilia much resemble that of the preceding; and like Vorticella, a group is generally rooted in one little mass of mucus: but every foot is capable of self-attachment, and individuals are constantly going off as free-swimmers; much more frequently than the preceding. Reproduction takes place in the same ways. There are other kinds in the family; and the Black Stentor is much shorter, and blunter at the foot, and always a freeswimmer.

Yet another colony form is found in various kinds of Ophrydium. The animals are vase-shaped and ciliated on the

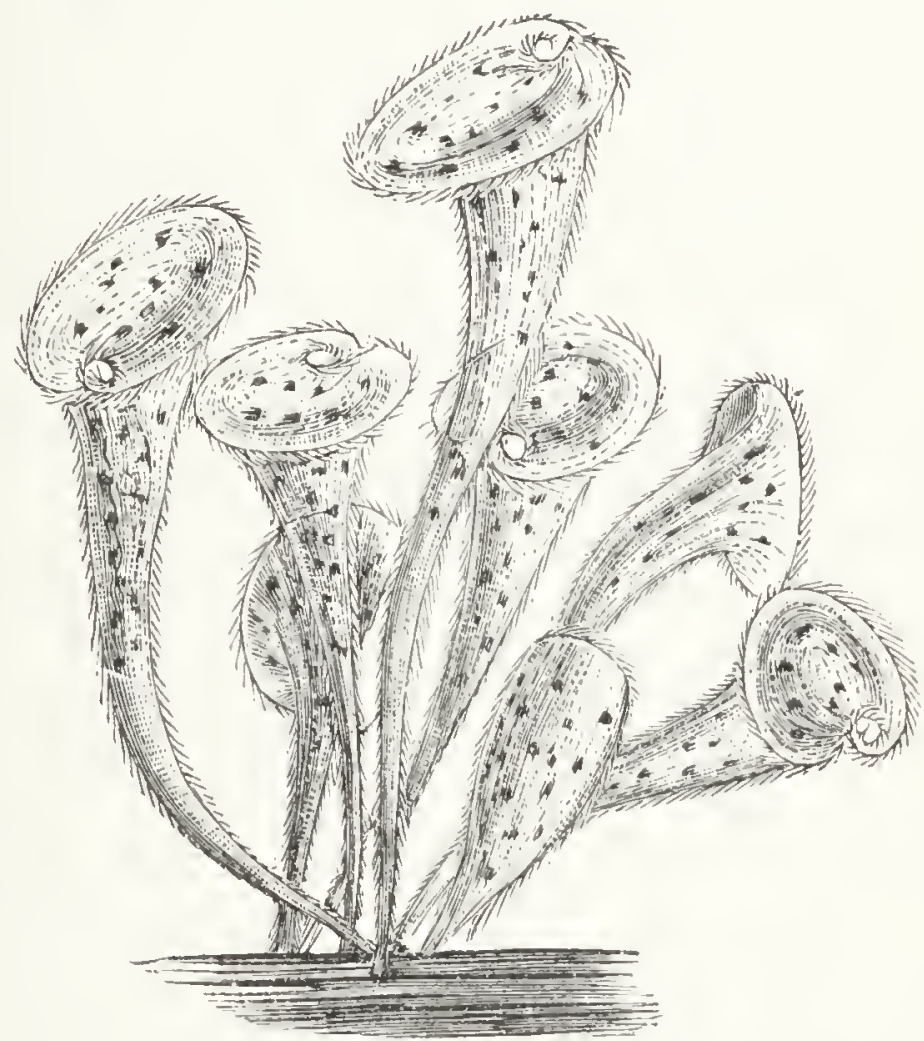

FIG. 88.-Stentor polymorphus $\times 40$ rim like Vorticella, but secrete a kind of jelly outside, by which a number of them are joined together, the ciliated ends protruding. The stalks meet in the centre. One variety is not uncommon in 'nice' ponds, in the shape of apparent balls of rather greenish jelly, in size from that of a small pea to that of a marble. This type will strongly remind us of similar groups of Rotifers, later on ( $\$ 88$ ).

We must look at one more type, generally considered the highest of the Infusoria, inasmuch as the young creature in 
some genera starts as a Ciliated Infusorian, but afterwards assumes another type. The class is called Tentaculifera, or the Tentacled Infusorians. The example before us is Acineta (fig. 89).' It is common enough on filaments of confervoid plants more or less loaded with decayed matter, but being only $\frac{1}{100}$ th of an inch in height, has to be searched for rather carefully: thus it differs also, in being a fixed creature. It cannot be mistaken, being known by its stiff stalk and the triangular shape of the cell; from the outer corners of which can be protruded or drawn in bunches of

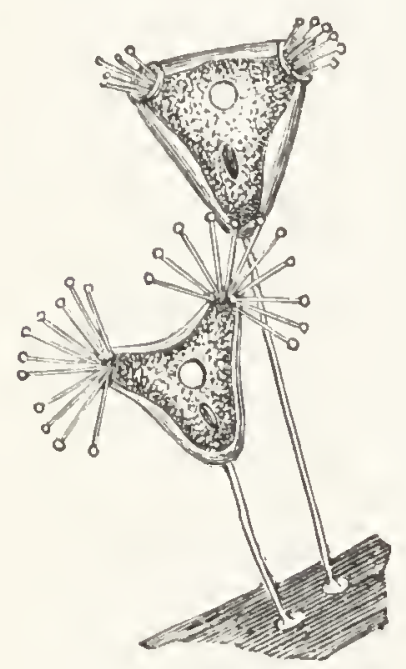

FIG. 89.-Acineta minute tentacles, with knobs at their ends. These knobs are suckers, and if one of them touches some small animalcule or other particle of food, others immediately come to assist. But they do not, like the cilia in other forms, sweep down the entire object into the mass below, where it might pass into a vacuole to be digested: they suck out the fluid portion, after which the rest is rejected. As the young are free swimmers, moving freely with the aid of cilia, the growth out of this larval form into another, is a step upward in organisation; and it is remarkable that in the somewhat rare Dendrosoma, many individuals in the adult form unite by their roots in a branched colony, showing also approach towards the Hydrozoc presently mentioned.

87. Volvox.-Before passing on to the more highlyorganised Rotifers, let us look at one of the most beautiful of all the forms of pond-life, Volvox globator. Its character is still debated. At first it was considered an animal colony: by the middle of the century it was generally classed as an assemblage of lowly alge or water-plants; now some

1 That here figured is a salt-water varicty; but the fresh-water type closely resembles it in all but being much larger. 
of the best modern biologists again class it as an animal; but it is also possible that the simple zooids of which it is composed may stand on the boundary line between the two worlds.

It is most common in clear pieces of water. It is a little capricious; and when none could be found in a likely large pond, I have found plenty in a small tributary pool surrounded by rushes, close by. But the invaluable ring and net will often find it in satisfactory quantities, when mere dips with bottle alone result in disappointment. A well-filled tube appears thickly studded even to the naked eye, with bright light-green specks floating about. To see them well, put them in one of the thinfronted troughs which has just space enough to let them move freely, but in one layer only, and use from an inch to halfinch power.

Fig. 90 shows the object in black and white, but this gives no idea of its beauty. The whole is brilliant orange-green

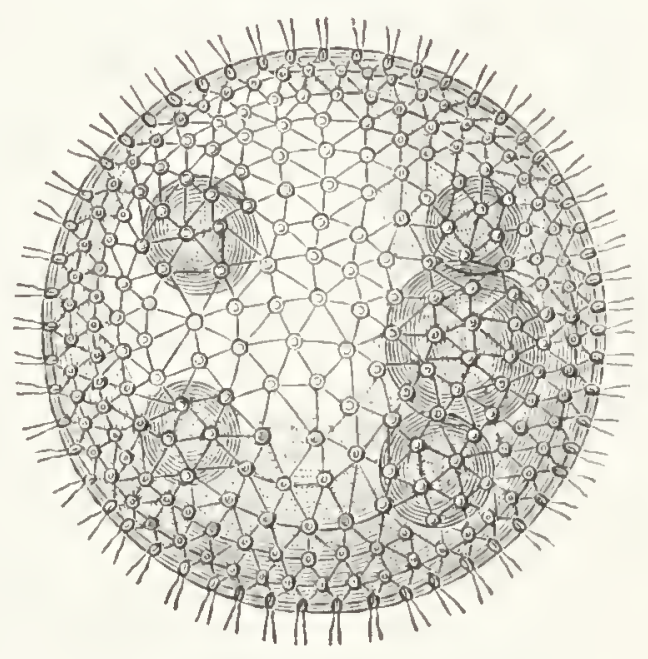

FIG. 90.-Volvox $\times 75$ tracery or lacework, like an open-knitted green silk ball kept stretched to a sphere by some invisible agency. This is in constant majestic ${ }^{1}$ rolling motion across the field of view. Inside the large sphere are several smaller spheres, seen to be precisely similar except in size, and which, if the large one is carefully ruptured by pressure, roll about independently, and would gradually increase in size. So much for the whole. More critical scrutiny reveals that each knot in the netted silk is really connected with the next ones by threads of transparent material (protoplasm),

1 Small as the spheres are, when once seen it will be felt that there is no incongruity in the term. 
but that each is, besides, furnished with two loose or free flagella such as were mentioned in \$ 86. These are easily seen round the edge of the great sphere, and by their motion the sphere is rolled along.

If by more pressure the Volvox is more completely broken up, it can be seen that each of these little zooids with its pair of flagclla, is capable of swimming about alone. We have in Euglenc, a single-celled green organism furnished with only a single flagellum, and which is so thick in some ponds as to give the water itself, apparently, quite a green

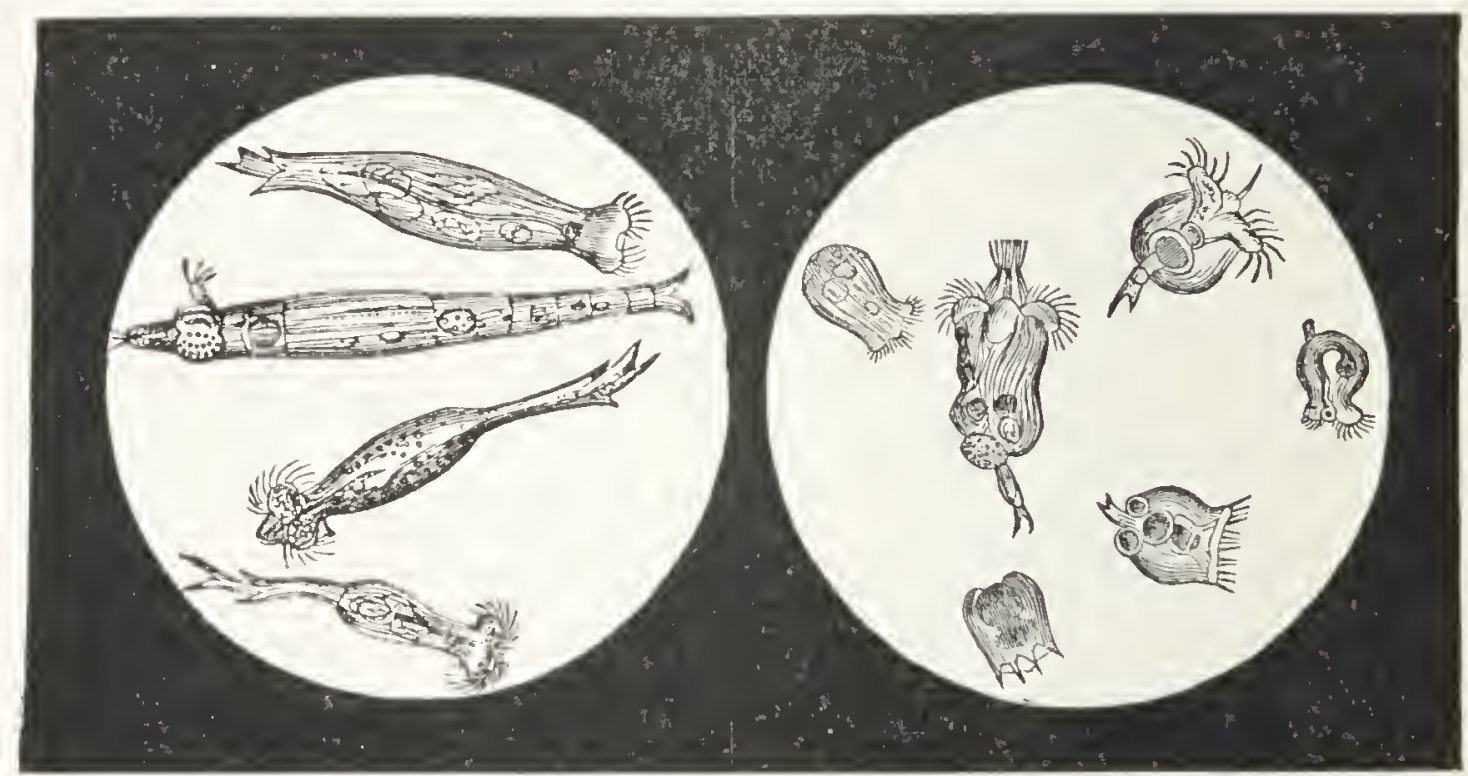

FIG. 91.-Rotifers

colour. This too has been considered both vegetable and animal, and there are not so many reasons for placing it in the latter class as in the case of Volvox. In any case the latter is to be considered a colony, composed of many singlecelled individuals.

88. Rotifers.-In the Vorticella, Stentors, \&c., we found advance from unsystematic cilia, to defined circles of them, producing vortices in the water, which swept food into the cavity serving as a mouth. In neariy all pond-water, besides such mere Infusorians, will be found a greater or less number of larger creatures, in which this feature is 
the leading character, and which are hence called Rotifers. But it is seen almost at the first glance, that they are of much higher organisation. They are of all sizes and shapes, but two special types stand out easily-the long and narrow, and the broader and pitcher-shaped, as shown in fig. 91.

The commonest type of the former is one in the above figure found everywhere, both in salt water and fresh, and known as Rotifer vulgaris, a more magnified view of which is given in fig. 92. This was the first to be observed, and has named the whole tribe. It shows at once that Rotifers are highly-organised creatures. There are evident signs of segments; from which the class is generally held to have some affinity with the worms. The foot with which most are furnished, obviously has toes, and is able to take distinct

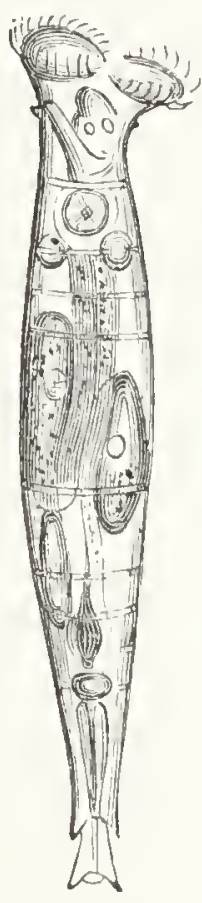

FIG. 92. R. vulgaris

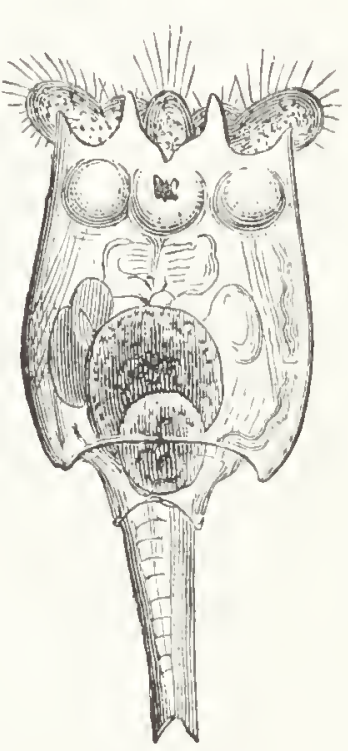

Fig. 93.

Brachionus grasp of fixed points. There is a distinct stomach and intestine; and there are a pair of powerful actual jaws, which work with a machine-like regularity to grind up the food, swept in by the vortex set up by the two sets of cilia. On most genera there are spots which it is thought fulfil at least some of the functions of eyes.

Fig. 93 shows a species of Brachionus, one of the short and broad type, very common in most ponds. The stem is very flexible, and this Rotifer and many others twist about on the foot in the most grotesque way; or the tail can be quite drawn into the sheath. There are, between the two ciliated discs, tuffs of setæ which do not move while extended. 
Amongst the many tribes, there are many more modifications of this kind, which we cannot describe in detail.

A characteristic class of these animals is known as the tubc-dwelling Rotifers, from the fact that the creature surrounds itself with a case or sheath, either of gelatinous matter secreted, or of pellets built up round it. Fig. 94 shows one of the Floscules. The tube is of transparent jelly, and the crown is divided into lobes which differ in

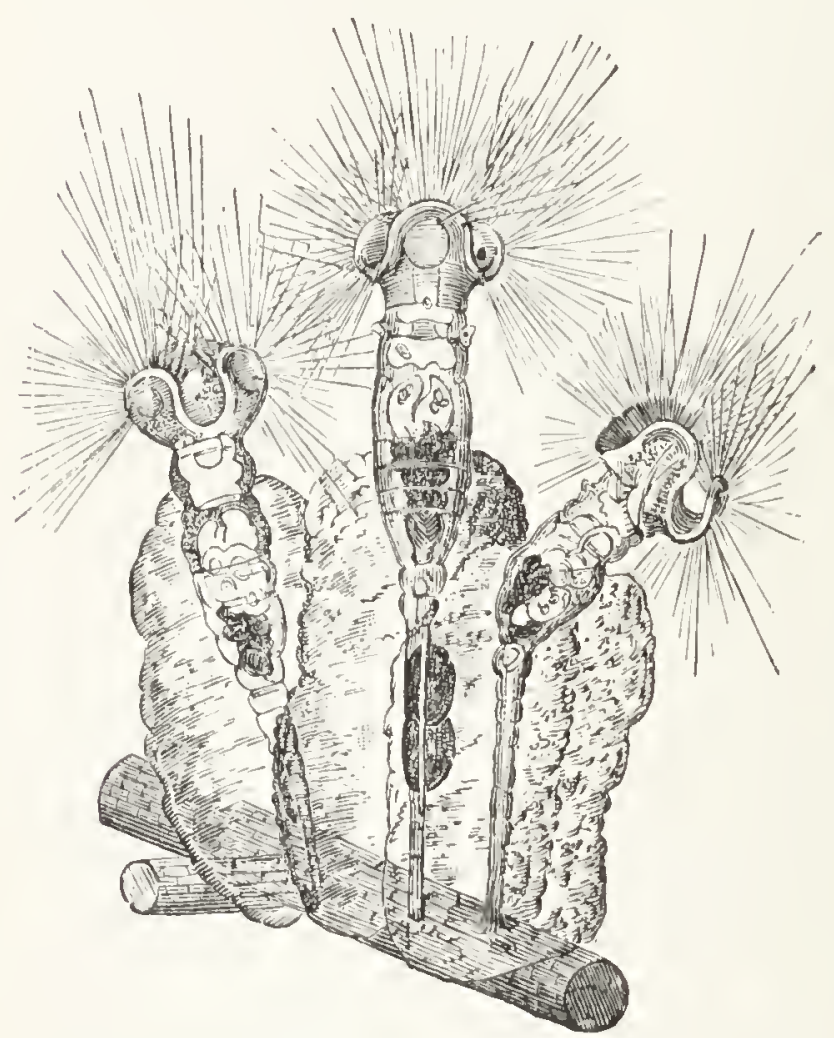

FIG. 94.-Floseules

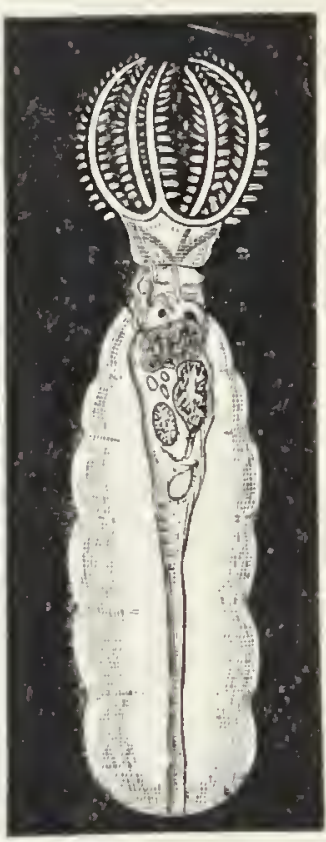

Fig. 95.-Stephanoceros

number with the species. From each of these lobes projects a radiating bundle of transparent straight filaments. Below these and the edge of the crown are the cilia. To exhibit the full beauty of a Floscule, will tax the utmost skill of a microscopist. It requires dark-ground illumination; and the more perfectly the optical conditions are adjusted, and the finer the lens, the farther and farther out the long filaments are seen to extend, far beyond what was imagined at first, and of almost inconceivable tenuity. 
Allied to the Floscules is Stephanoceros, which also secretes a transparent tube. It however is furnished at the top with five curved processes, armed with cilia all the way up, and which spring outwards and then curve inwards again, like the petals of a flower. This is a great favourite, and is also displayed to best advantage on a dark ground. It is generally found on the weed Anacharis or the filaments of confervæ, and is very easily seen in a glass tube with the pocket magnifier. Floscules on the other hand are hard to find. I have found both Stephanoceros and the Melicerta next mentioned, breed very freely in the micro-aquarium, and have had long filaments of Spirogyra studded thickly with Stephanoceros from end to end.

The interesting brick-building Rotifer, Melicerta ringens, is easily seen with the naked eye; and few large masses of Anacharis can be searched long without finding it. When at work it protrudes four circular lobes with cilia, which look like revolving cog-wheels; but it is very shy, and the least shake to the table makes it drop back into the tube. It looks like a bit of brown pack-thread $\frac{1}{20}$ th of an inch long (when fully

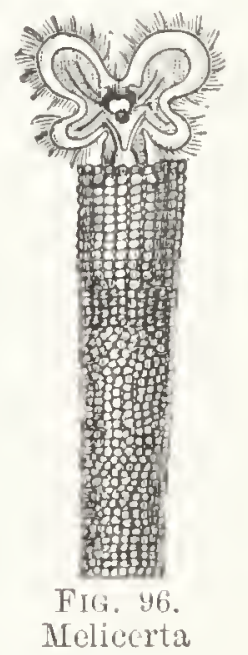
built up), sticking out perpendicularly from some small leaf or stem, and with apparently the very tiniest morsel of something like thistledown at the top. Under the microscope, the brown tube is seen to be made up of little pellets or bricks : and as close observation is continued, each little pellet can be seen being ground up and formed in the little mill nearly in the centre of the four ' wheels.' When complete the pellet is seized, and the upper portion of the animal bends over and deposits the new brick on the top of the wall it is building. There are some allied varieties; but this is both the commonest and most interesting.

We can only mention in the last place the clustering or colonial. Rotifers, which are combined in masses bound 
together by a centre of gelatine, like the Infusoria of similar type (page 154). All are plainly allied, though the shapes of the ciliated crowns differ a little, and also the precise mode of attachment. The most beautiful is perhaps Conochilus volvox (fig. 97), in which the individuals are gathered together at the feet by a central lump of jelly, into a spherical ball, much in the fashion of a child's cowslip-ball. The whole group revolves through the water like Volvox (to which of course it is in no way allied), but every separate Rotifer is quite capable of separate freeswimming existence. The drawing is from a beautiful slide mounted by Mr. Rousselet. Both Megalotrocha and Lacimularia are usually attached to some plant or twig, and

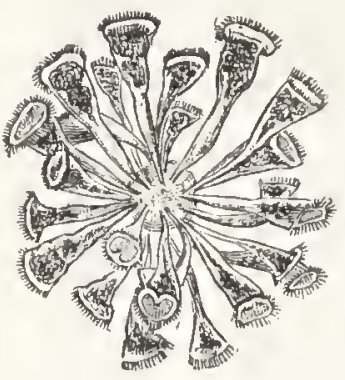

FIG. 97.-Conochilus

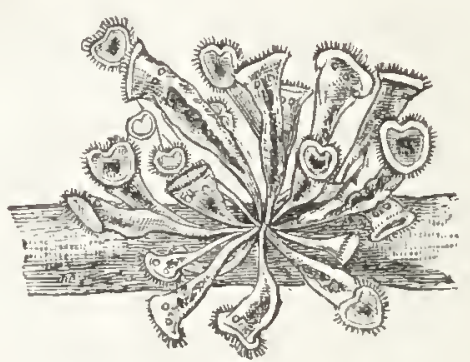

FIG. 98.-Megalotrocha

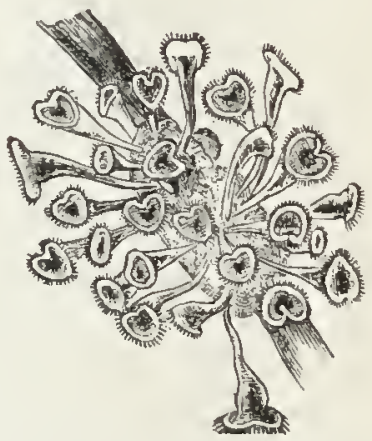

FJG. 99.-Laciuularia

are found in running water, whilst Conochilus is found in ponds. In Lacimularia there is a somewhat large central lump of jelly, (fig. 99) whilst in Megalotrocha the feet only are attached and centred at almost a point (fig. 98). The latter may also be known by having four opaque white spots set round the neck, or space under the ciliated corona.

It is interesting to see in these different forms of clustered Rotifers, an evident instance of adaptation to somewhat different conditions. The two last mentioned are capable of revolving in a free condition; and a small new colony often passes some time in that way. It is obvious that in still water this plan of existence offers advantages, both in respiration and finding food; whereas in running water, 
which of itself brings food and air, the balance of advantage must be in a fixed position.

These clustered Rotifer's are seen to the best effect on the dark ground with a low power, needing as they do much 'depth of focus' to see the entire group fairly. They are also beautiful objects treated as opaque, by rays from either' the bull's-eye, parabolic reflector, or Lieberkühn.

89. Hydrozoa. - We have in this class of animals quite another type, of which the fresh-water Hydra is the general pattern. There are three varieties of Hydra: the common, a browner form, and the green, or Hydra viridis, with much shorter and more clumsy tentacles, looking rather like a small budding flower. One or the other can be found in almost any pond where there are plants or duckweed, to which it fixes itself by a sucker at the foot. Fig. 100 shows the Common Hydra; but it differs a great deal in appearance. After a full meal the tentacles are drawn in and blunt: when food has been scarce, the ar'ms may stretch out as thin threads over an inch long. From time to time little buds form as at B, which grow into branching Hydras. There are also eggs at certain seasons.

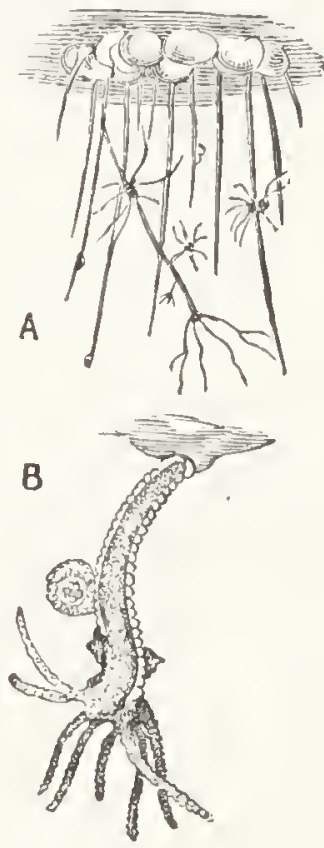

FIti, 100.-Hyilra A. Natural size B. Lnularged

In one respect the Hydroids are very low and simple animals. There is no stomach or intestine, only a mere bag. On the inside of this are simple ciliated amoboid cells; and when the food has been dragged down into the cavity by the tentacles, particles of it pass into the vacuoles of these to be digested, just as in the lowest forms of life $(\$ \$ 85,86)$. The stomach is a mere sort of colony of amœbæ. Hence it is that the Hydra is able to reproduce itself from pieces, as everybody knows; but it is not the fact that the outer skin will do for a stomach if turned inside out, as well 
as the inside; it is a skin, not furnished with amøba cells, and has to be turned back again if the creature is to live.

But this simple stomach colony is served by a complicated apparatus. Under the microscope the tentacles are seen to be studded with suckers, as shown in B, fig. 100; and if one of these touches a small Rotifer or Infusorian, the latter appears to be paralysed, and in a moment or two is dead. Even Entomostraca are usually killed in a few seconds; but I learnt in a curious way that a strong one, owing to the resisting power of its shell, may sometimes escape. A gentleman told a friend of mine that he was giving a lecture on Pond Life to an audience of soldiers, illustrated by the

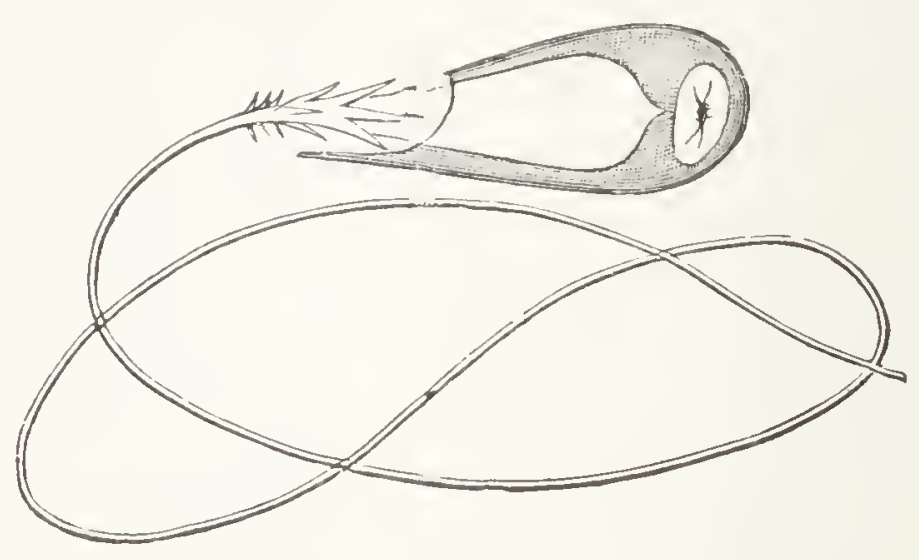

Fitr. 101.-Threal cell of Hydra viridis
Lantern

scope, and a living Hydra was upon the screen, when it grasped a waterflea. The latter, instead of being paralysed as usual, made the most violent efforts to escape, turning over and over, amid excited murmurs from the soldiers, who looked on with intense interest, the power of the Hydra having just previously been explained to them. At last the Daphnia hurled itself free; when suddenly every man in the room sprang to his feet and joined in a deafening cheer-the queerest experience, the lecturer said, he ever had!

This deadly effect is produced by 'thread-cells,' with which the tentacles are thickly studded, and the nature of which is shown in fig. 101. Each consists of a little sac or bag filled almost to bursting with a poisonous fluid; and coiled up in the fluid is a long thin filament; the filament as well as the fluid is therefore in a state of tension. Con- 
tact or pressure ruptures the sac, when both fluid and filament are forcibly ejected, the end of the filament entering the victim, and piercing it ready for the poison. The small Hydroids are not powerful enough to incommode large animals; but Medusx or jelly-fishes sting in precisely the same way, and it is well known that their sting amounts to agony. They belong to the same great class as the Hydra; and it is remarkable that many of the Hydrozoa, particularly marine forms, throw off young which are meduse, and swim about in the same way. The thread-cells of the Hydra can generally be made to eject for examination by adding a little magenta or iodine to the vater in the trough.

There is another single polyp in the tanks of several Botanic Gardens, which has a beautiful medusa form also: it probably came on some plant from South America. In Cordylophora, which Mr. Scherren says is becoming commoner in England, we have a multiple or colonial form, many hydra-like heads branching away from one stem. Marine forms are numerous (\$ 92).

90. Polyzoa. - These are amongst the most beautiful pond subjects. They bear much the same relation to multiple Hydrozoa, as a colony of Rotifers to one of Infusoria: that is, the individuals making up the colony are highly organised, with a stomach and other organs. In mere outward appearance, some marine Polyzoa greatly resemble some Hydroids; but the internal organisation places them upon a higher plane.

Only six fresh-water kinds are often found. Alcyonella was once called the fresh-water sponge, and forms in spongy masses round sticks or roots rather deep in the water, in both clear ponds and streams. A small slice cut out will display the beautiful flower-like zooids, with their circular crowns of ciliated tentacles. There are several forms which branch like a tree, Plumatella being one of the most beautiful. Two others have each zooid enclosed in a membranous 
and gelatinous mass. Fig. 102 shows one or two individuals of a colony of Lophopus crystallinus, with their crowns of waving tentacles. ${ }^{1}$ The most remarkable however in some respects of all the Polyzoa, either in fresh water or salt, is the Cristatella mucedo, which when fully developed has an extraordinary resemblance to a thick hairy caterpillar. A single polypide can start a colony, which at first is a small

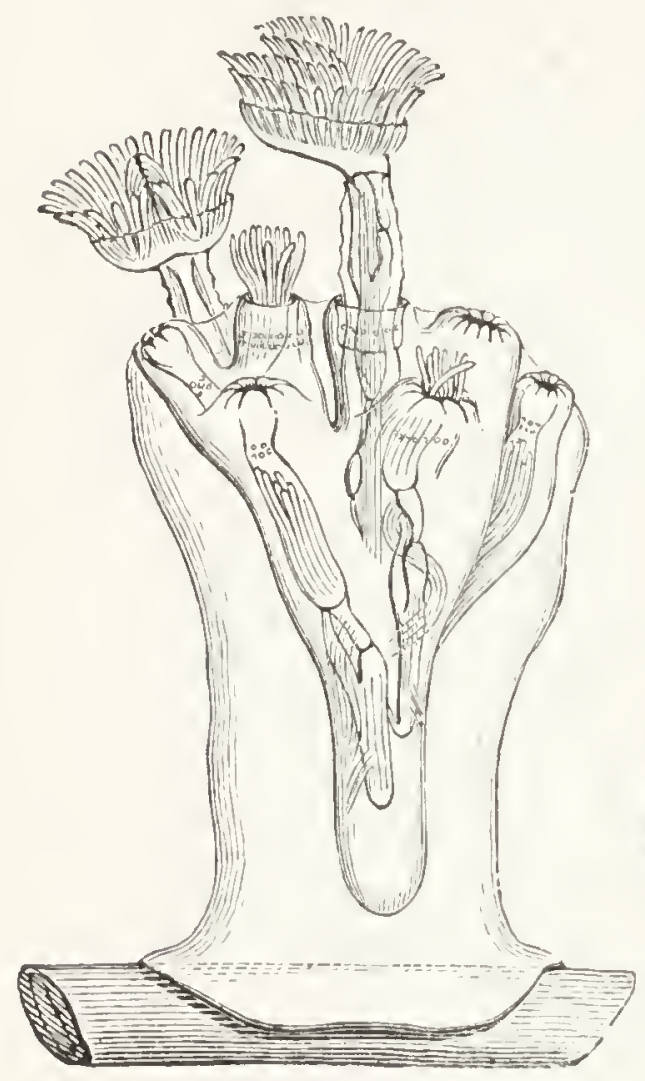

FIs. 102.-Lophopus spherical group : but this gradually increases in length. The remarkable point is, that after awhile the entire group assumes a determinate form, of a long oval shape, flat underneath and

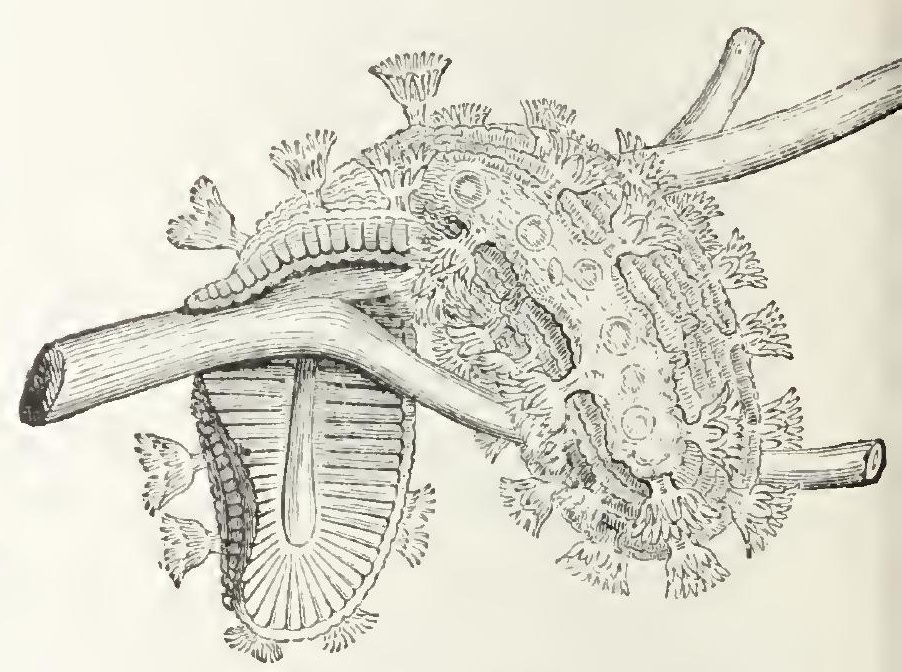

FIG. 103.-C'ristatella mucerlo $($ Allman $) \times 5$

convex above. The polypides are found arranged in three rows round the back, leaving a space up the middle in which the statoblasts or eggs are developed. And the under side assumes a regular fluted structure, which travels over the pond-weed or twig like the foot of a slug! This is very wonderful and even mysterious; for in some respects the undoubtedly independent individuals appear to be combined

1 The drawing is from Allman : but is far too stiff and rigid. There is a flexible curling beauty about the crowns, as they are withdrawn or protruded, of which this drawing gives no idea. 
into a sort of single animal, which moves as a whole by common consent, and possesses functional parts (in the foot) which cannot be said to belong to any of the single polypides. This problem cannot be said to be understood. The whole is like a bed of living flowers, and its beauty can be imagined. Fully developed colonies are rare, and chiefly found in autumn in ponds well lit by the sun: the other Polyzoa are mostly found deep, in more or less shady pools. The condenser or spot-lens best brings out their exquisite beauty.

91. Miscellaneous Pond Life.-The Desmids, Algæ, and other lowly forms of vegetation are left for Chapter $\mathrm{X}$. Space does not permit us to mention in detail the many objects furnished by larval forms of Insects which inhabit the water. The larva of various gnats and other small insects are interesting objects. One larva known as Corethra plumicomis, or that of

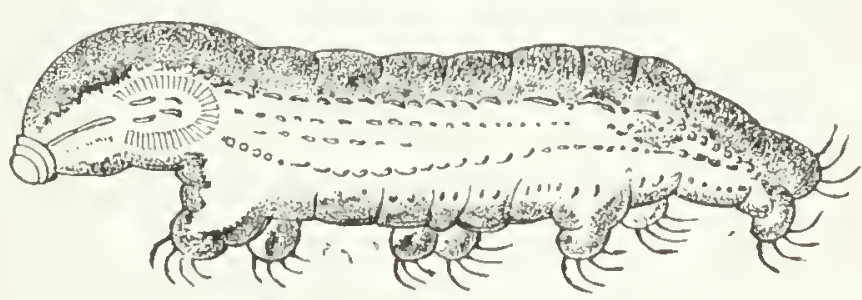

Filk. 104.-Water Bear the plumed gnat, is as absolutely clear and transparent as glass, and under a low power all the internal organs can be examined. Another beautiful object is the larva of the Mayfly, which is furnished on both sides with many sets of gills, by which oxygen is conveyed to the air-tubes.

Many small worms are also well worth examination. Standing out of the mud will often be seen clusters of red threads waving about: these are the small worms called Tubifex. The whole digestive and circulatory apparatus is evident. Shown on the screen by a good lantern microscope, the creature looks like an immense python, gaudily coloured, and ten or more feet long.

There are also interesting varieties of water-mites and water-lice. The most curious of the former is perhaps the very small creature, not larger than some Rotifers, known as the Watcr Bear. Except that it possesses four legs 
on each side, it does look ridiculously like a bear, as it crawls about and pokes its bear-like snout into the débris on which it feeds.

92. Marine Life.-A harvest of beauty is to be reaped by the seaside, but the ground already gone over will enable us to be very brief. The net will be as useful as ever in obtaining small crustaceans, in greater numbers and variety than fresh water affords. It will also obtain plentiful variety of salt-water Rotifers and Infusoria; and on

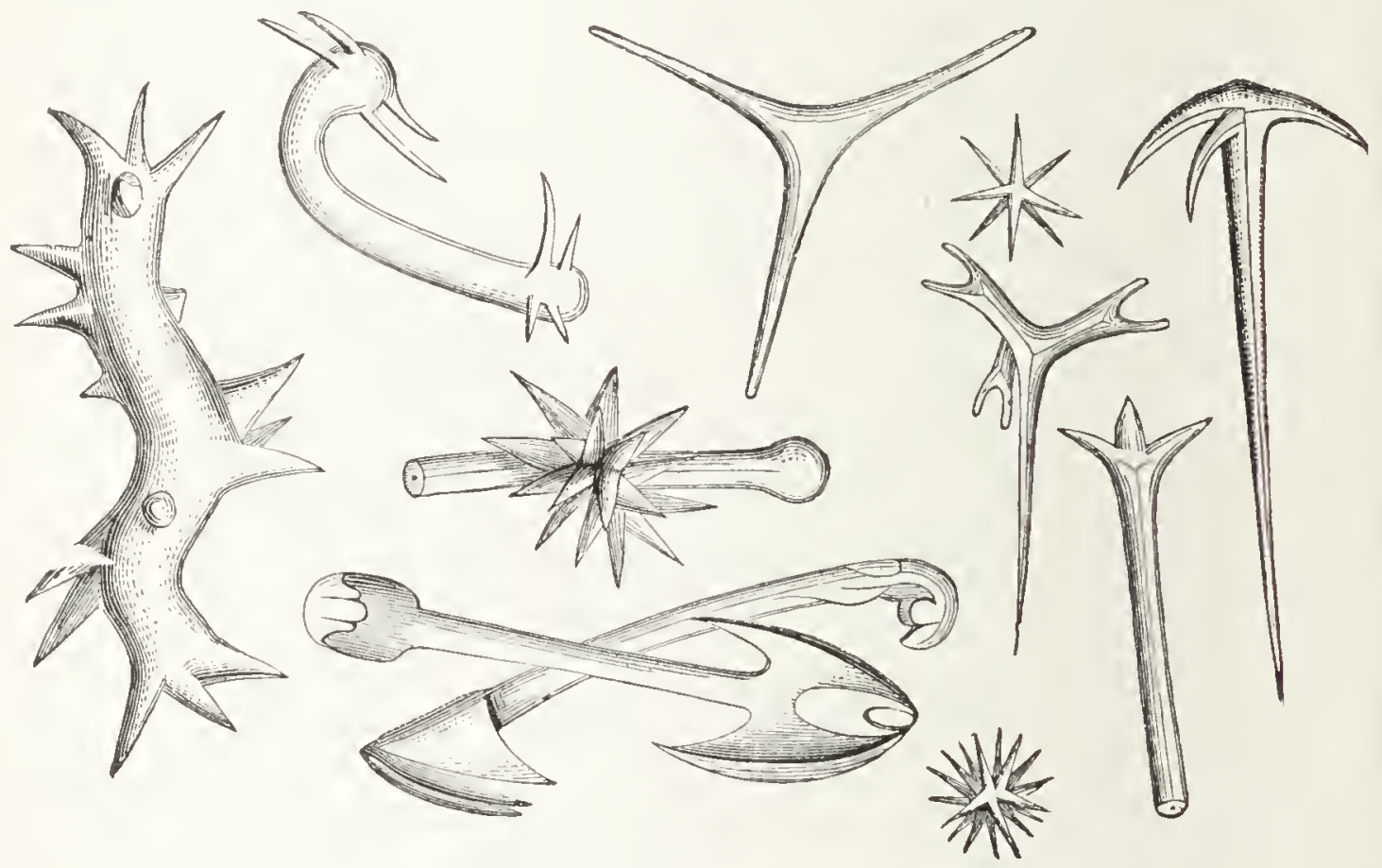

FIG. 105.-Spicules of Sponge

many parts of the coast, skimming of the surface will obtain various marine diatoms. There are also many beautiful forms of minute vegetation, more fully dealt with in the handbook already mentioned.

Sponges are a study in themselves. There are one or two fresh-water forms of interest, but the majority are marine. It hardly need be said that sponges are now regarded as colonial animals. Most of them are plentifully furnished with a kind of skeleton of calcareous, or more generally silicious Spicules, which of themselves make 
interesting slides. They may be all joined into one skeleton, as in the beautiful Euplectella or Venus' Flower Basket; or they may unite as a looser but connected lattice-work; or (more generally) be detached. The forms vary greatly, from the simplest needles, to types of which a few only are given in fig. 105. Somewhat similar spicules are found amongst Echinoderms, and the 'anchors' of Synapta and 'wheels' of Chirodota are wellknown microscopic objects.

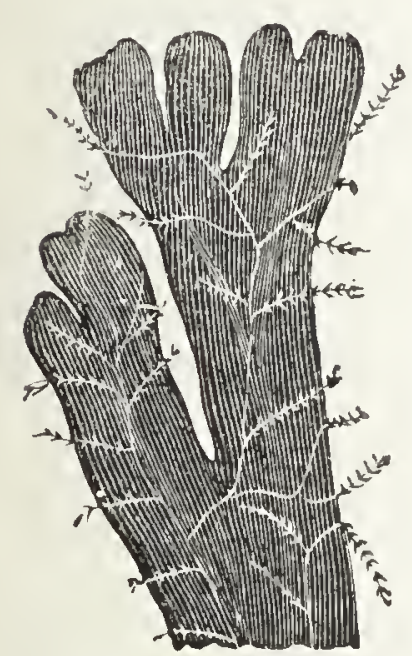

FIG. 106.--Sertularia pumila (naturat size)

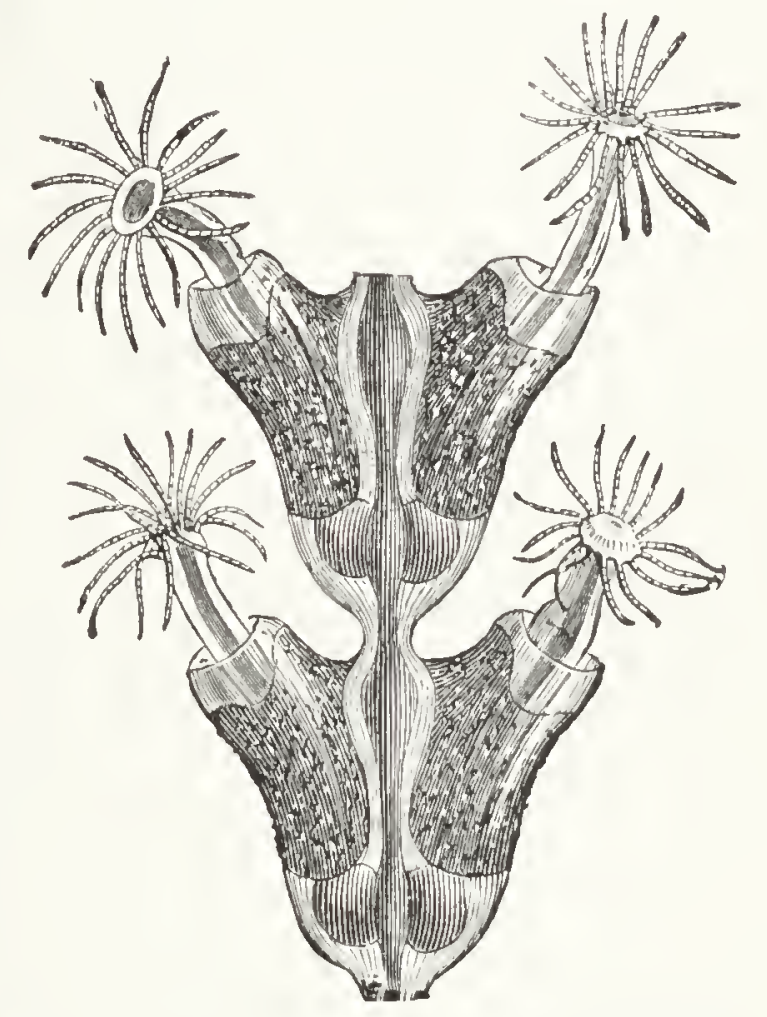

F1G. 107.-S. pumila (maynified)

But the greatest variety, and the easiest of the more beautiful subjects to obtain, will be found in the marine Hydrozoa and Polyzoa, which are far more plentiful than the fresh-water forms. Some must be sought in rockpools, in rather dark cavities, or under the sea-weed: some will be got by the drag-hook (fig. 73) from depths too great to reach otherwise: some are only obtained by dredging from a boat. But a great deal may be gathered from shallow pools, especially the little pools in sand formed by the swish of the tide round points of rock; and many beautiful forms can be obtained by merely turning 
over the sea-wash, or loose sea-weed thrown up by the tide, whilst still wet and fresh. Let us take only an example or two of what the tyro can be certain to get almost directly.

It is impossible to search long amongst sea-wash without finding a bit of sea-weed with some tiny growth upon it like fig. 106. Place a little bit in a trough, under an inch or two-thirds, and the beautiful Sea-oak Coralline (Scrtularia pumita, fig. 107) appears, each polypite appearing like a tiny flower, one on each side of each joint of the branching stem. Quite as common in most places is Coryne fruticosa, shown of the natural size $(a)$ and mag-

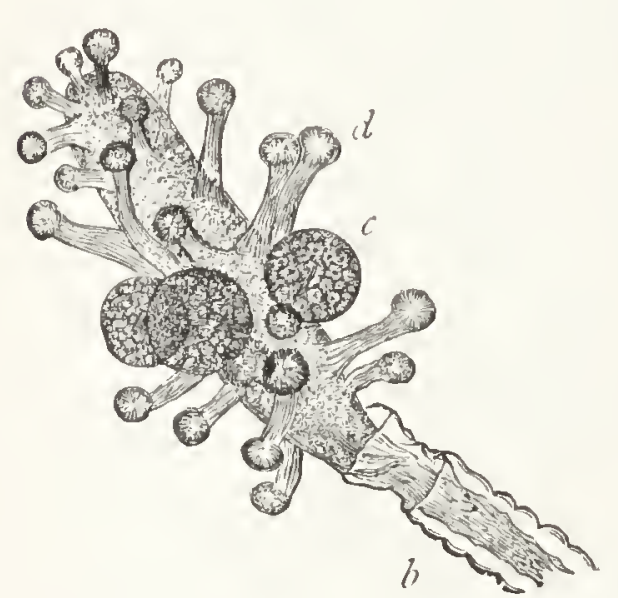

FiG. 108.-Coryne fruticosa

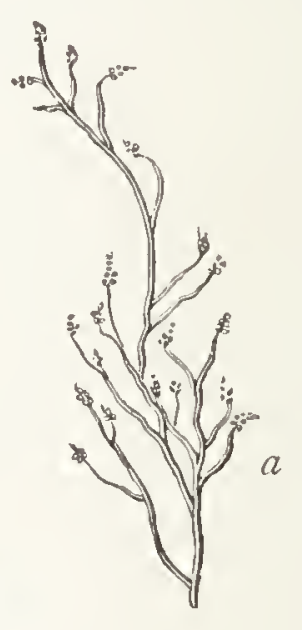

nified The tentacles here are seen to be of another type, with globular heads and shorter, and not so free in motion as the preceding. These club-shaped heads are veritable armouries of the stinging 'thread-cells' noticed in the case of the Hydra, and common to the tribe: also there may generally be found some gonophores (c) or reproductive scions. Great interest will be found in observing these differences, and comparing the polypites, their mode of arrangement on the stem, their grouping \&c., which cannot here be indicated in detail. The last of the Hydroids we will mention is the pretty Luccrnaria (fig. 109). This is usually found on sea-weed or Zostera about (or rather outside of) low-water, and is shown here about the natural size: under the microscope each of the eight arms is seen to have a beautiful tuft of tentacles, which paralyze prey in the usual manner, while between 
each pair of arms is a smaller organ which serves as a sort of foot or anchor when the creature moves about, as it is constantly doing. This beautiful Hydroid is not so common as the foregoing.

At least one of the marine Polyzoa is as plentiful as the common Hydroids. This is the pretty Nit Coralline. It abides in rather deep water, outside low-water mark as a rule, on sea-weeds there found, and especially on the pretty delicate pink weed itself known as the common Coralline. But I have often found it in sea-wash after a storm. The figure shows the appearance to

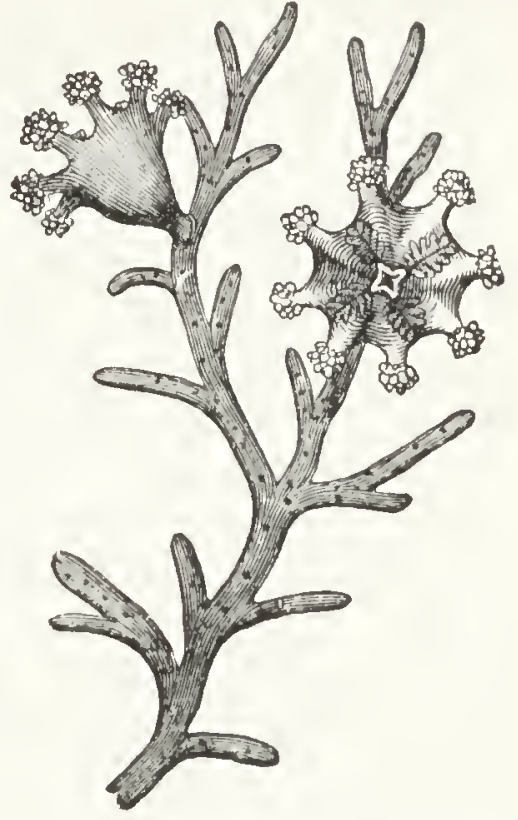

Fit. 109.-Lucernaria unaided vision, and when magnified; the spectacle under the microscope being of wonderful beauty. The distinction in character of the Polyzoa will be at once seen, in that amidst a considerable general resemblance, we have obviously a greater importance and complexity of the individual animal.

Here however we must conclude this chapter, in the hope that some readers may find sufficient interest in Pond and Marine life to follow up the study with more of

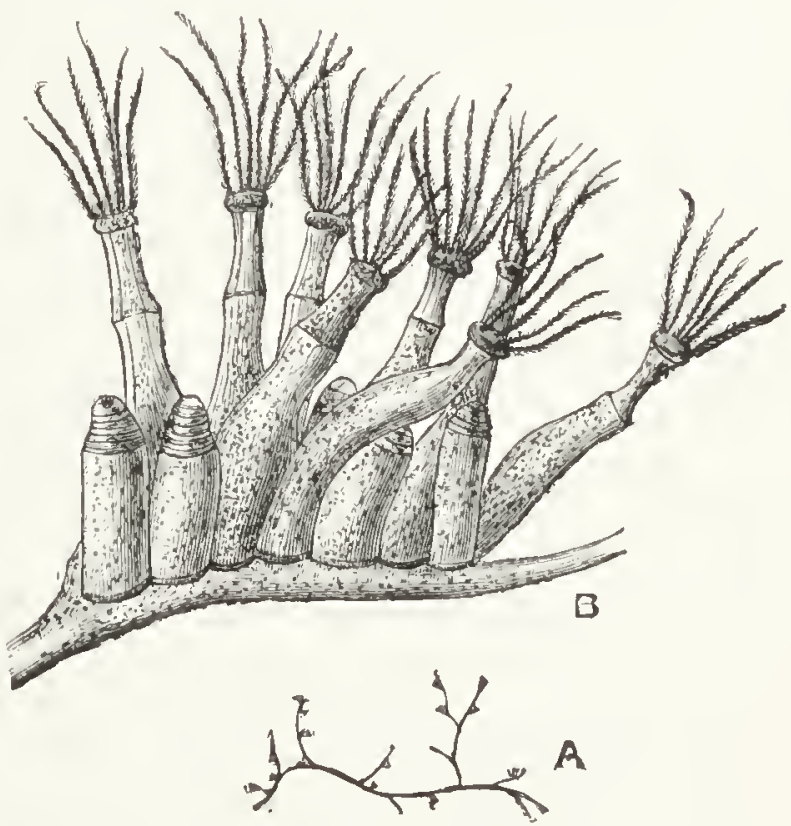

Fiti. 110.-Nit Corulline A. Nitural size B. Magnifiel method and detail. 


\section{CHAPTER IX}

THE INSECT WORLD

Next to pond and marine life the Insect World probably opens to the microscopist the most generally attractive, extensive, and easily accessible array of subjects. The ease and facility with which these may be prepared for preservation, has been indicated in Chapter VII.

93. The Study of Insects.-We would specially urge the student to make general observation of the head and mouth organs of the living insect, in the manner indicated in $\$ \S 94,97$. They are so utterly different from all ideas he may have gained from animals, that in no other way can an intelligent conception of their arrangement, and extraordinary modifications, be attained; and every one who has thus examined a few of the leading types under a low power--from 1 inch to 2 inches-will have acquired an additional range of ideas, and an amount of realistic knowledge, which will impart to his further studies in detail the highest interest.

Our next advice is to carry out microscopic work, as opportunity offers, upon some definite system. This will wonderfully add to the interest from the very first; and as the system is carried on, will maintain it to the very last. It may be done in several ways, of which two only need be mentioned as hints for a beginning. We may set out to study one inseet all through, or at least as regards its more obvious parts and organs. Or we may, on the other hand, set out to study similar organs as modified in different insects -as for instance wings, legs and feet, mouth organs, dc. It is obvious that the two plans may be happily combined; and that by pursuing either the microscopist will gradually gather a really valuable collection, instead of a mere hetero- 
geneous collection of slides. Let us take an example of each method.

94. A House Fly.-The common house fly-with reference also to the Blow Fly as larger, but otherwise nearly identical in structure-will serve well for the study of an insect, and we can indeed not indicate a tenth of what it offers for study; if anyone desires an idea of what there is to study in an insect, let him refer in some library ${ }^{1}$ to $\mathrm{Mr}$. Lowne's grand monograph on the Blow Fly. We must take a more general view of the creature, shown as by a strong pocket magnifier in fig. 111.

From this we see its place among Insecta, since its many sedgments are plainly 'cut into' three divisions of head, thorax, and abdomen. We notice its apparently single pair of wings, as distinguished from two pairs in many insects: thus classing it among the Diptera. We can perceive in general its large compound

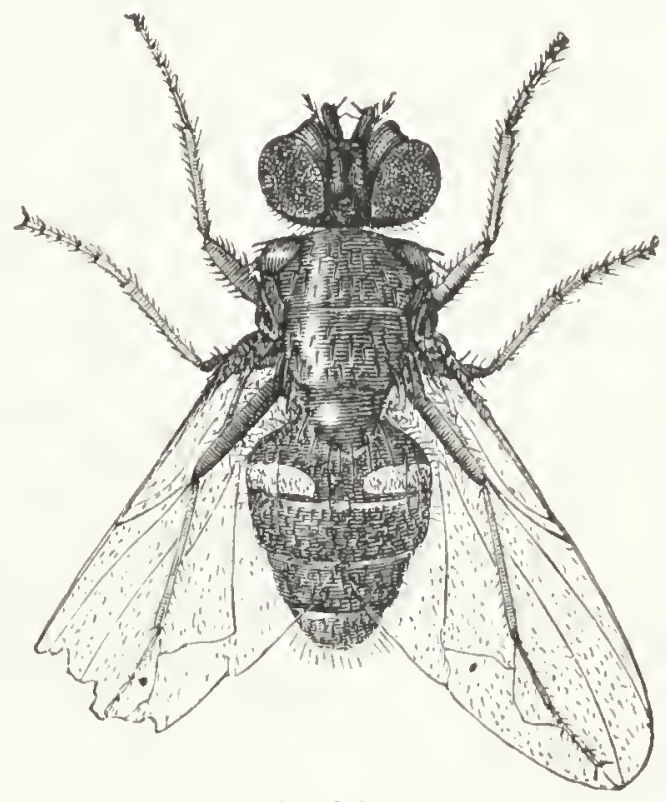

FiG. 111.-House Fly eyes; and with a little care can see the spots on the sides of its thorax and abdomen, which are the spiracles, or openings of the air-tubes. Its three pairs of legs are plain enough, and mark off true insects from spiders and mites.

A very little dissection, also under a low power, ${ }^{2}$ will show how totally the plan of an insect differs from that of a vertebrate animal. The latter has an internal skeleton: the insect has an outside one of horny substance called chitine,

1 The book costs, I believe, three guineas.

2 Insects can be killed for dissection in a wide-monthed stoppered bottle in which is placed either: (1) Some fresh chloroform on wool. (2) Or some fresh pounded laurel leaves, covered by a piece of perforated card or zinc. A cyanide bottle is more powerful, but somewhat dangerous to have about. 
to which its muscles are attached. The great nervous chord runs in animals along the back: in insects it is along the front. The animal's circulation is from a central heart through arteries and veins: in insects there are several chambers or hearts, near the back, which drive the blood forward, not through veins, but through the spaces between various organs. And instead of localised lungs or gills,

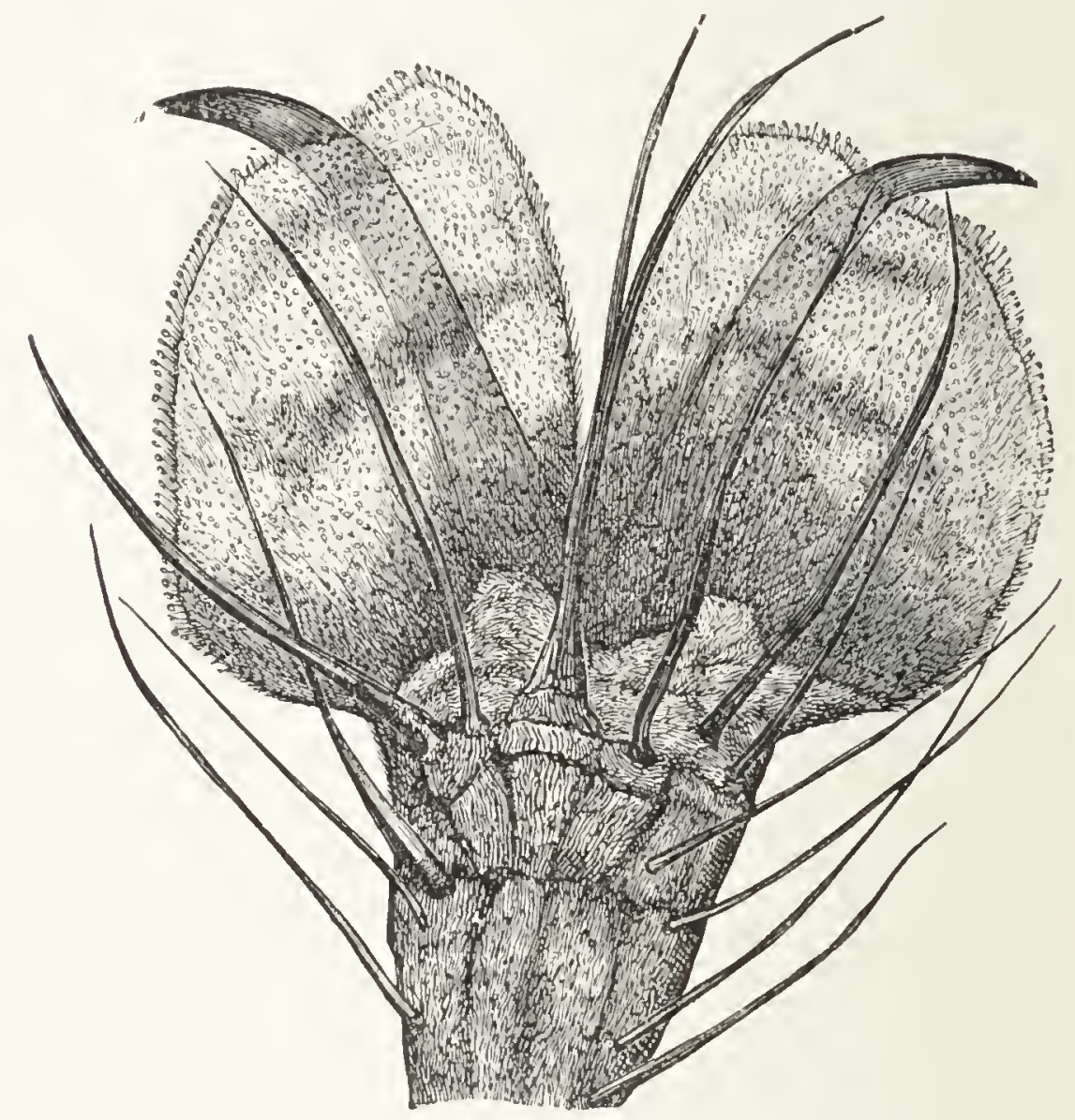

Fili. 112.-Foot of Fly

the whole body is traversed by a net-work of air-tubes, with ever so many open entrances along the sides, through which the blood is refreshed with oxygen.

Let us now examine some of the parts and organs under higher power. And first for the legs and feet. The leg consists of many joints (see \$95) which are studded with pretty long and coarse hairs. At the foot the most notice- 
able features are, first, the double claw which is general in nearly all insects; and secondly, the two pads or pulvilli, which are by no means so. It is by their means that the fly can walk upon glass; but how this is effected can hardly be said to be certain even at the time this is written. For years we used to read that the pads were suckers holding on by a vacuum; but it was conclusively shown that this at least was a mistake. Since then it has been generally believed that the small hairs with which the pads are covered, and which appear to have small disks or knobs at the tips, exude a sticky fluid, by which the fly sticks on. Though such hairs would let go as the pad was ' rolled' off the surface, it would take a little force to do this ; and so in winter, when the flies become feeble, they may often be found stuck by their feet to the window. It is also a fact that they do leave a little sticky matter behind them. In 1895, however, Mr. Merlin, using lenses of higher class and wider angle than had been usually employed upon this wellknown object, claimed to have discovered that the knobs or disks resolved themselves into very fine curved hairs. His observations have been confirmed by Mr. Nelson and other's, and it is possible that in many cases these curved hairs may act as very minute hooklets, in the same way as the caterpillar prolegs described on p. 181. But it is hard to see how adhesion to glass can be obtained in such a manner; and my own observation is that while many hairs, especially round the edges of the pad, can be resolved as described, there are other's which cannot be so resolved; and that in the feet of some flies-such as Asilus for instance-whose parts are coarser and easier observed, the knobs or disks are unmistakeable. The question cannot be regarded as settled at the time this is written; but the case is mentioned as a good illustration of how much work yet remains to be done, with modern improved apparatus, upon even objects supposed to be well-known to every one. 
The wing consists of a double membrane, exquisitely thin and transparent. A moderate power will show that it

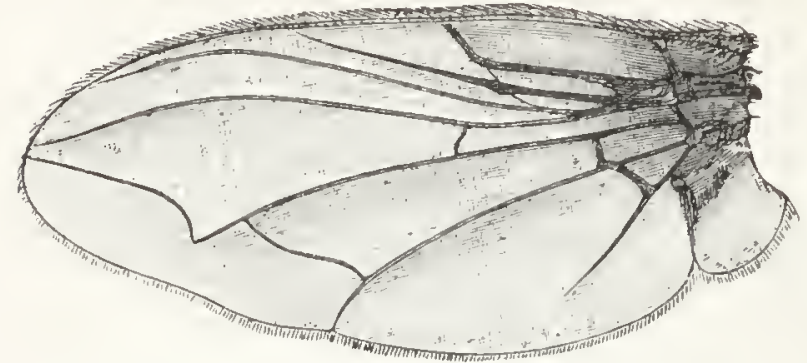

$\operatorname{Wing}(\times 5)$

Winglet $(\times 10)$

Balaneer $(\times 10)$

Fig. 113.-Wing of Plow Fly is thickly studded all over with small hairs. The veining is of course conspicuous, and may be compared with that of other dipterous flies. This is really the forewing only. At the base of the posterior edge is a curious double semicircle called the winglet or alulet: when the wing closes one of the semicircles slides in over the other, and the fore-wing over both. This however is not the rudiment of the posterior wing, nor is its function clearly known : the real hinder wing is represented by the little rod with a knob at the tip, also shown. The pair of these are called hatteres or balancers.

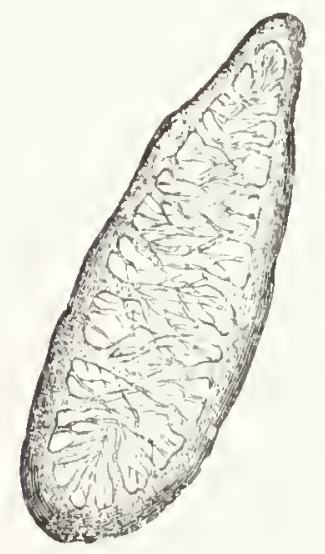

FIG. 114.

Spiracle They have some such function: but there are some minute structures and nerves at the thickened base, which will tax the highest powers of the microscope, and which have been thought to signify sense-organs of some sort: here again is further work yet waiting to be done.

In the body, we must content ourselves with the respiratory system. A bit of the skin with an air-opening or spiracle is easily dissected out and mounted: it is well to take one from both thorax and abdomen. Fig. 114 shows one from the thorax, which is a pretty object, and shows how the opening is guarded by a sort of sieve-like arrangement from the entrance of any coarse particles. Fig. 115 
shows a portion of the branched net-work of tubes which communicates with these openings. These tubes have spiral threads of chitine to strengthen them, precisely as wire is coiled inside or outside of rubber tubing for the same purpose, as shown on a larger scale in fig. 116. The structure of these air-tubes is more conspicuously seen in those of larger insects; but a portion of those of our fly can be teased out and mounted without any difficulty.

Turning now to the head, the proboscis (fig. 117) is perhaps the most familiar object in every collection. The

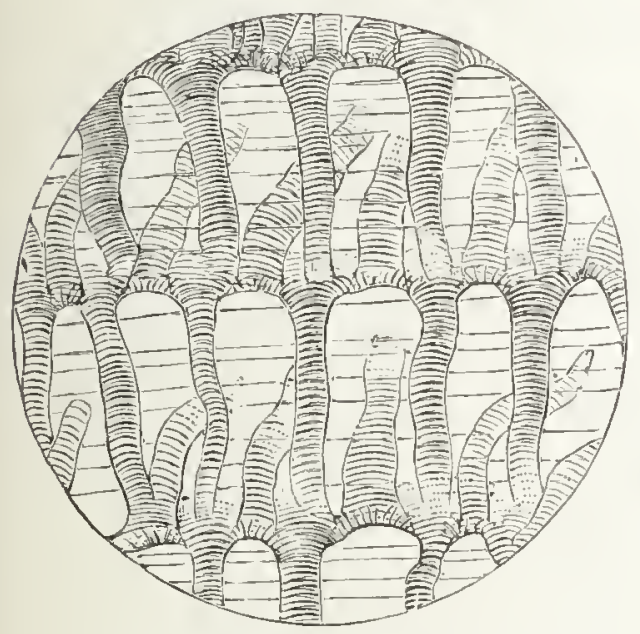

FIG. 115.-Tracheal Tubes

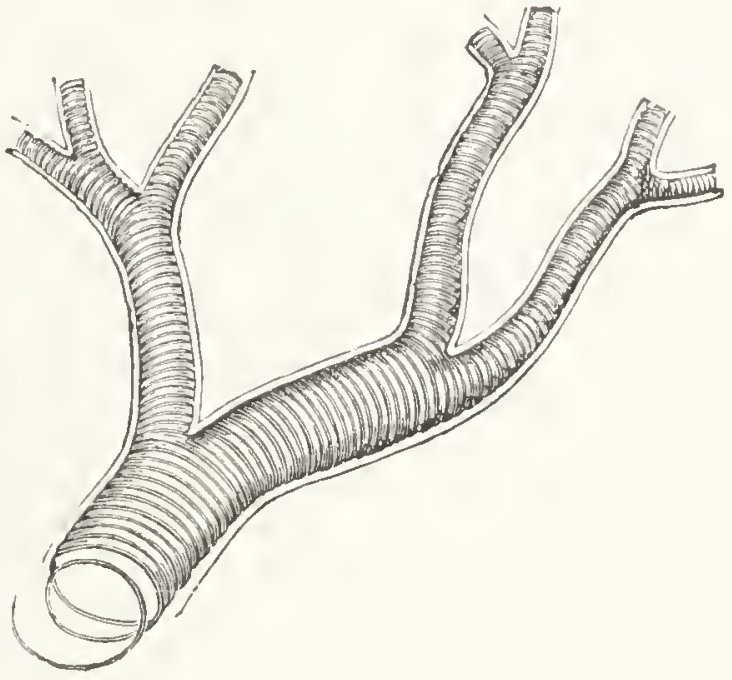

FIG. 116.-Tubes more magnified

figure shows the organ squeezed out flat, which displays well the haired maxillary palpi, mp, the powerful muscles and tendons, the fleshy lips $l$ with hairs round the margin, the very minute delicate hairs which stud the whole membrane, and whose tips are tests for the very best lenses, and especially the pseudo-tracheæ or sucking-tubes, $t r$. These are guarded by chitinous rings as in the trachex, but not of the same structure. There is no continuous spiral; but each ring is detached, and does not go all round, the ends being alternately slightly thickened, and of horseshoe form, as shown at $\mathrm{B}$ in fig. 118. It seems probable that fluid can be sucked up through the entire fissure caused by the opening 
between the ends of the rings, which opens on the face of the proboscis; and it is also possible that the hard ridges may have a scraping action: but here again certainty has hardly been obtained.

There are also minute sharp piercing instruments, which are however hidden by this mode of mounting, and are in this fly smaller than in many other Diptera. The proboscis

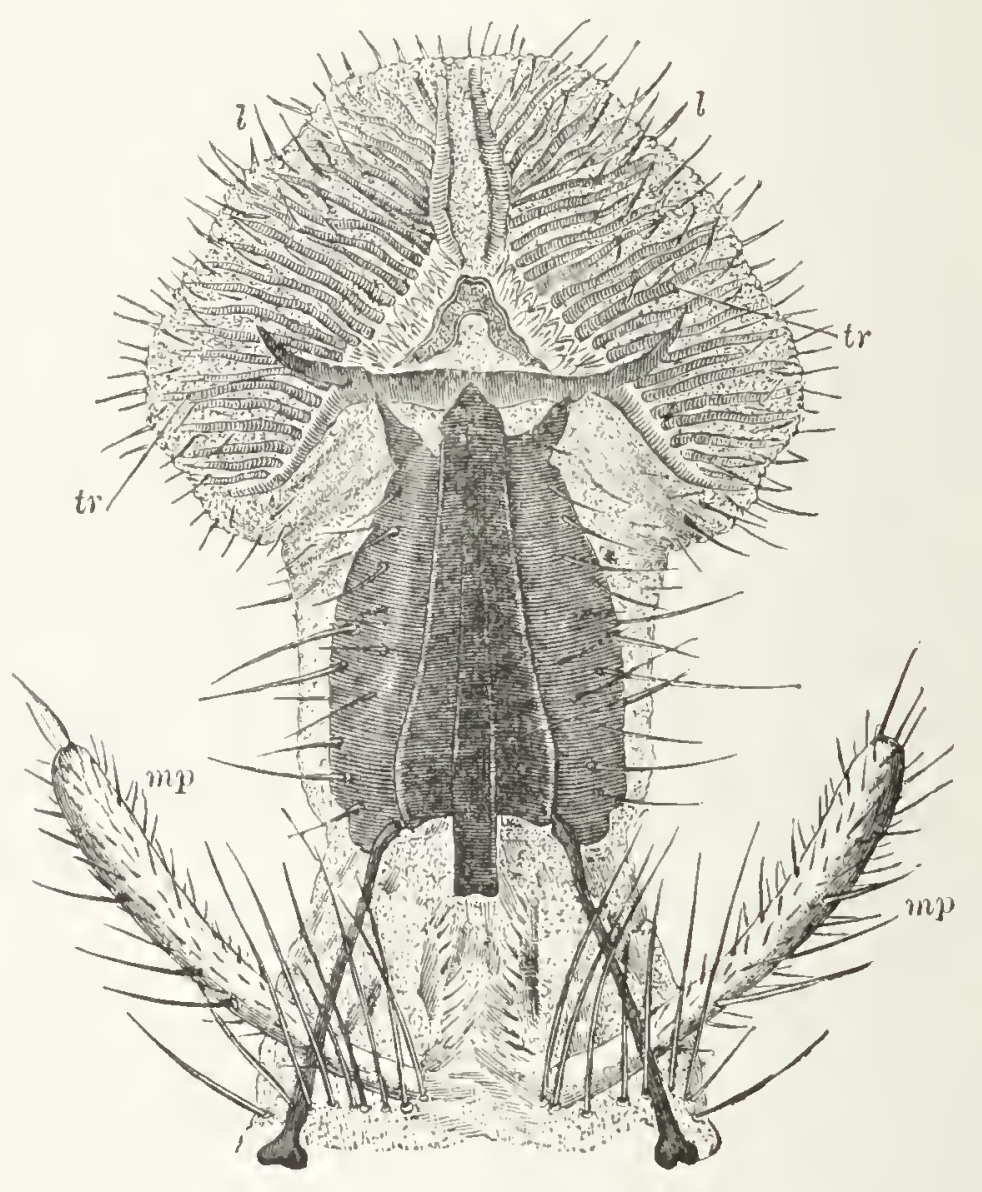

FIG. 117.-Proboscis of Fly

should also be examined from a side-view, preferably without pressure, for the general relation of parts, and for these piercing organs, which are far more developed in some other flies (\$ 97).

Fig. 119 is the antenna. This has six joints, much less than in many insects; but some of them are too small to be discerned except under the microscope. They are numbered 
in successive order in the figure. The third joint is peculiar, and will tax the highest powers. Under these the surface is seen to be studded with minute sacs covered with a fine membrane. A somewhat similar structure is far more highly developed in the flattened antenna of the Cockchafer. It is plajn that such delicate organs must minister to some delicate sense; but whether that sense is hearing, smell, feeling, or some extra sense inconceivable to us, is by no means certain-here again is work still to be done.

The eye is amongst the most remarkable features. In the case of many insects, the great bulk of the head consists of two immense compound eyes, as may well be seen in the

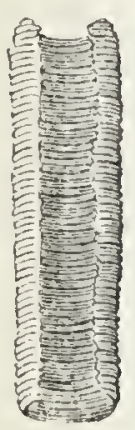

A

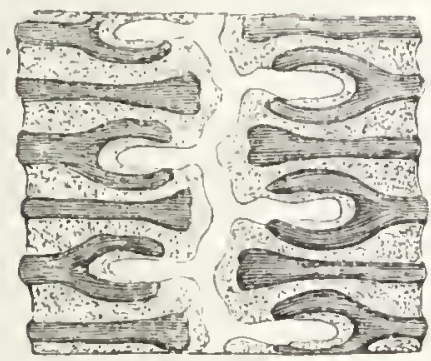

$\mathrm{B}$

FIG. 118.-Sucking Tubes
A. Tube
B. Rings magnified

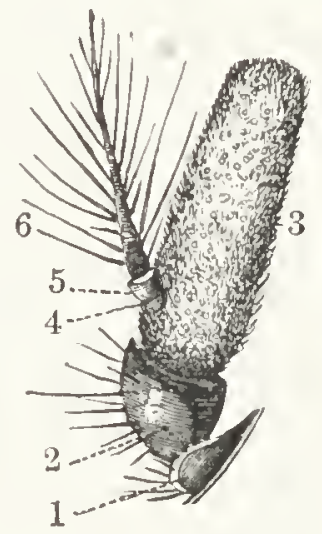

Fig. 119.-Antenna

head of a Tipula (daddy long-legs). Each mass is seen to consist of thousands of facets, every one of these being a separate lens, focussing the image of the tiny bit of the world immediately in its axis, at the foot of a crystalline cone behind the lens. All rays but a small axial portion are stopped by a diaphragm of black pigment. The general plan of a fly's eye is shown in fig. 120, the nervous rods whose ends take the place of a retina being expanded at their tips near the base of the cones. The precise arrangement differs in various insects. In most flies the lensec have outer convex surfaces; some being plano-convex, and others double-convex. In the eye of the Water-Boatman the 
outer surface is plane, and the inner convex. In the eye of the Cockchafer the lens consists of two parts, an outer lens and an inner one, in contact. The lengths and shapes of the cones also differ.

To cut good and thin sections of these compound eyes will tax an experienced section-cutter ; but it is easy to wash away the rest of the eye from the cornea of lenses, with a camel-hair brush, and mount this cornea flat, snipping the edges a little, that the spherical surface may lie down. The set of lenses of a large insect, such as a water-beetle or

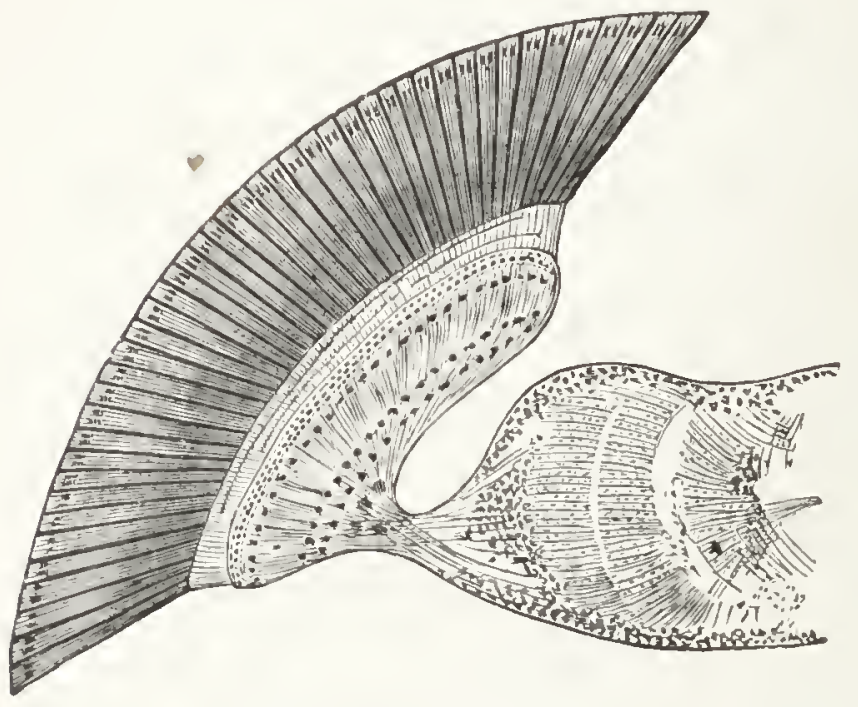

Fig. 120.-Eye of au Insect dragon-fly, will exhibit the focal power of the lenses, by showing multiple images of the source of light. The best plan I find to be to place in the substage (without a condenser, and racked as far back as it will go, close to the mirror) some bright coloured object. Then focus first the cornea itself. On now racking back the objective carefully, this will go out of focus, while there will appear many small images of the luminous object. This is a pretty experiment; but it does not convey a true idea of insect vision, or of anything at all beyond the fact that these tiny lenses arc real lenses. It has been shown by actual experiment, that when an insect's compound eye is used to take an actual photograph in its natural spherical condition (which has been done by Professor Exner) there are not many small images, but that one large image is truly pictured.

Besides these compound eyes, many insects have several single eyes on the top of the head, between them. Three is 
the most usual number, and these are set in a triangular form. In our fly they are very small; in wasps and droneflies they are conspicuous. The relation of these single eyes to the others, and to the total vision of the insect, is at present a great mystery.

Here we must leave our fly, with much about it not even alluded to. There are the digestive system, the muscles, the nerves, and many other details which will all repay study; but we must pass to another point of view. Let us now suppose that the student desires to make comparisons, and study resemblances and differences. As we began about the fly with its feet, let us take for example of this method, a few specimens from the legs and feet of other insects.

95. Legs and Feet of Insects.-First take a couple of larval forms, one quite general, the other much the reverse. Fig. 121 represents the feet of the Goat-moth caterpillar, a general type of many other caterpillars. The top figure is the end of one of the 'pro-legs,' which are mere membranous projections from the skin, a pair springing from each of four segments, and another pair from the last segment of all. The end or foot is furnished with a row of recurved hooks all round; and it is the hold of these hooklets upon the skin, which causes the 'creepy' feeling when a caterpillar crawls on the hand. All these 'legs' totally disappear' with the larval stage; and the three pairs

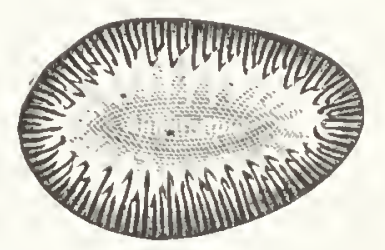
of real legs are represented by the short-jointed organ, with a single claw, shown in the lower part of the figure.

Not legs in the proper sense, but certainly locomotive organs of a peculiar kind, are shown in fig. 122, the very minute larva (magnified) of Tanypus maculatus, a small two- 
winged fly, found in the flocculent vegetable matter at the bottom of ponds. It is very transparent, and a beautiful object; but we have only to do with the peculiar organs shown, projecting from a segment behind the head, and at
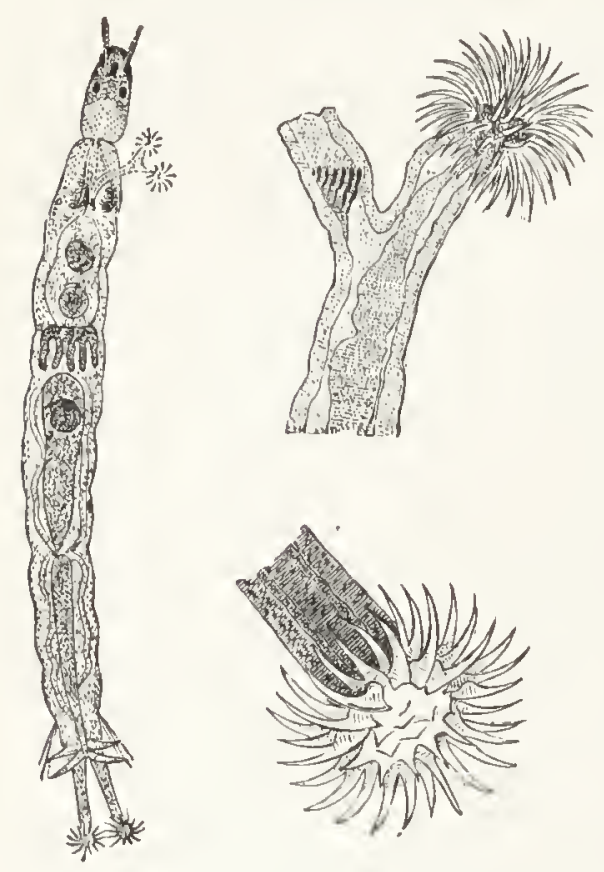

FIG. 122 the 'tail.' At first nothing may be visible; but gradually a little projection comes out, then this forks into two, and finally from the end of each starts a mass of little hooklets, as shown in the upper of the more magnified figures. In the other fork these are shown drawn in, as the whole can be at will by the muscle seen passing down the centre of the branch. The pair at the tail are very similar, as shown in the other enlarged figure.

Going now to adult insects, a normal example will be the leg of a cockroach, which shows the five parts. First is the

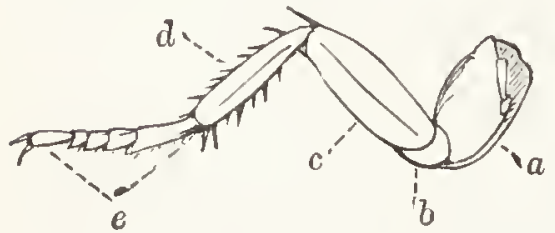

FIG. 123.-Cockroach leg coxa, $a$, next a small joint called the trochanter, $b$, next the thigh or femur, $c$, then the tibia, $d$, and finally the tarsus, $e$, here and normally with six joints and ending in a double claw, which ends the limb in the great majority of cases. Leaving the host of beetles and other insects which have legs closely adherent to this type, we will look at some curious developments and modifications.

Perhaps the most striking are amongst the water-beetles. Fig. 124 is the foot of the fore-leg of the male of the great water-beetle, or Dyticus marginalis. Here the first three joints of the tarsus are expanded into a broad surface, which 
is fringed all round with curved hairs. From the surface itself spring a great number of short hairs with cup-shaped disks at their extremities; the greater number small, but one very large indeed, and another (in some varieties two others) of intermediate size. All have practically the same general structure, as shown in the more magnified view

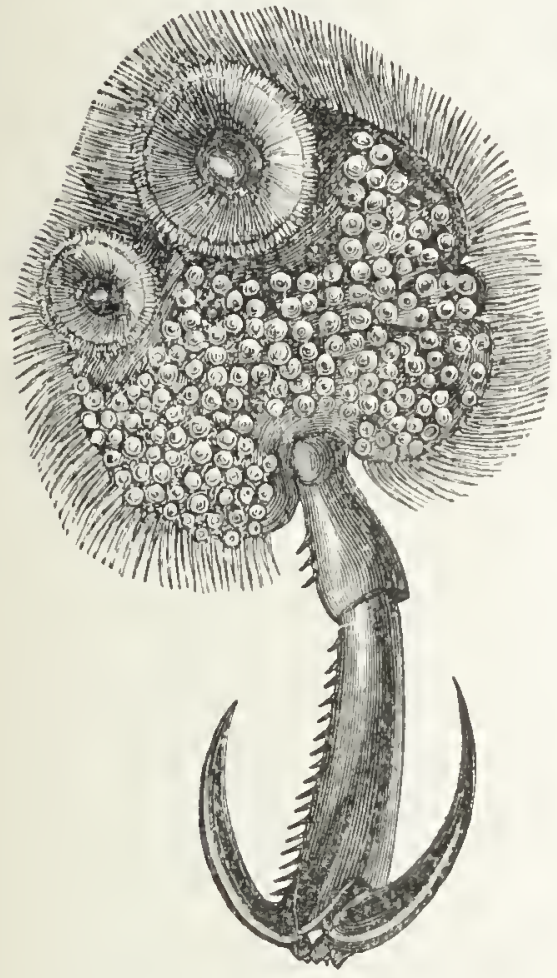

Fig. 124.-Tarsus of Dyticus given in fig. 125. Ridges of chitine go from centre to circumference of the cups, and the idea of their being 'suckers' is almost irresistible; but it has been all but proved that they are not, but simply apparatus for conveying a riscid adhesive fluid, and in fact a further development of the hair's

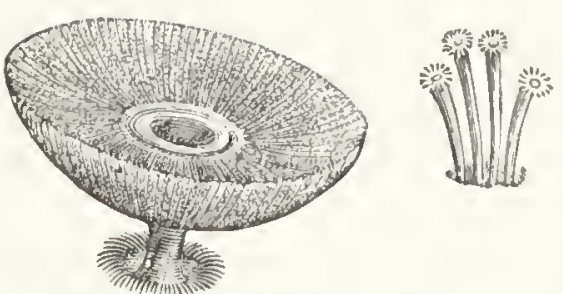

FIG. 125.-Hairs with Disks

mentioned in describing the foot of the fly, page 175. The stem of the large 'sucker' is easily overlooked unless looked for and examined sideways. The other two joints are terminated by a double claw, in this case unusually large. The hind legs of the same insect are also worth mounting, for their immense fringe of long and thick hairs, which act as a propelling paddle when expanded.

Our next example is the expanding paddle of the Gyrinus or Whirling beetle. The front pair of legs are of the ordinary type, but the hinder pairs are peculiar (fig. 126). The trochanter $t r$, femur fcm, and tibia (tib) are flat plates of 
triangular shape, jointed at outer angles, from which the apex of each next plate springs. But the tarsus (tar) is jointed on

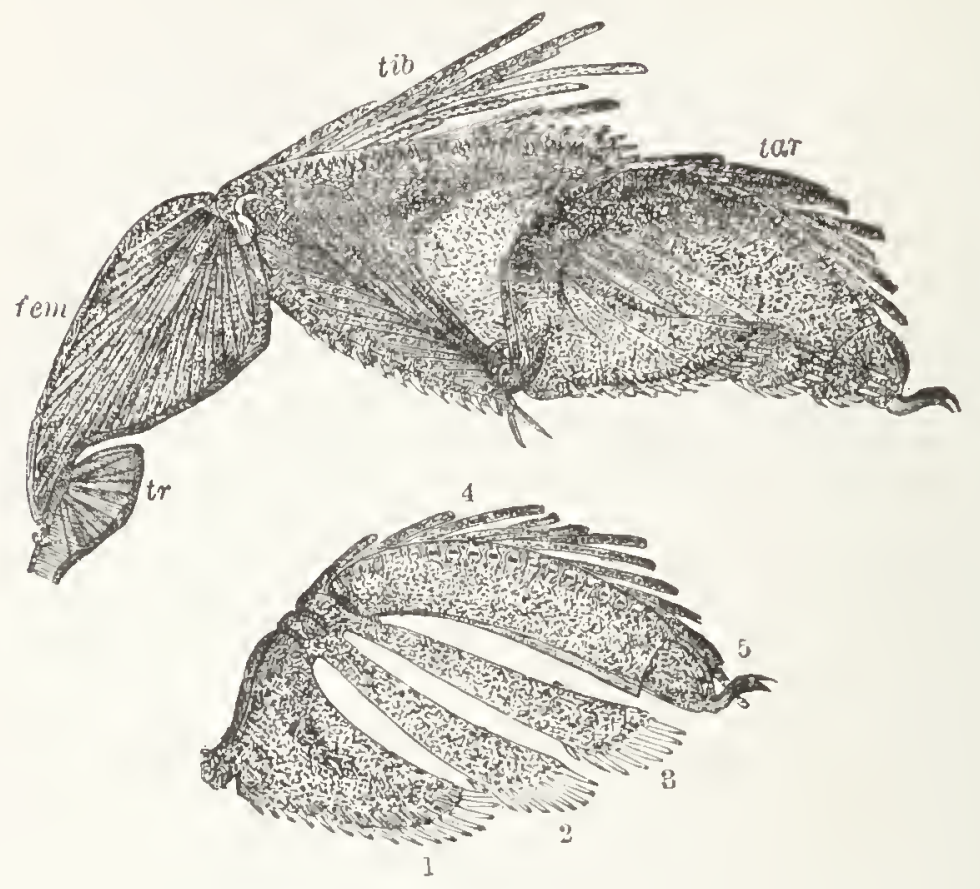

Fig. 126.-Paddle of Gyrimus

at the inner angle of the outer end of the tibia, and each of its four joints is expanded on one side into a flat paddle - blade. These are shown nearly closed in the upper figure, and expanded and more enlarged in the lower, where the joints of the tarsus are num-

bered. The paddles are moreover fringed with flat hair's which further increase the surface. The fourth joint bear's a small fifth one with its usual double hook.

Fig. 127 is the foreleg of the Water Scorpion. Here the femur $f$ becomes by far the largest joint, containing muscles of comparatively tremendous power. These muscles close the tibia like the blade of a knife upon the femur, which is grooved like the handle of the knife to receive it. The claw alone

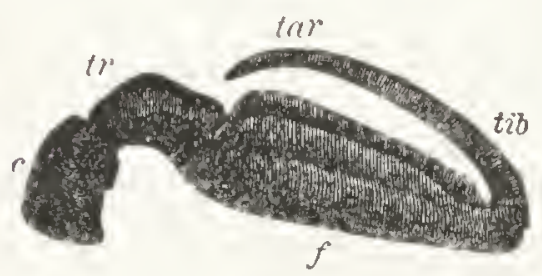

FIf. 127 here represents the tarsus tar, and is not jointed. This powerful instrument is employed by the insect to seize and crush its prey.

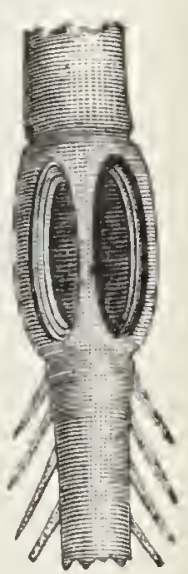

FIG. 128

Fig. 128 shows the tibia of the foreleg of the Great Green Grasshopper, which is remarkable for the pair of orifices, 
oval in shape, near the upper end. These orifices are in direct communication with the main tracheal tubes which pass down the limb. Such a remarkable feature must have some special function; but this has not yet been discovered. It is possible that other instances of the same kind might be observed if search were made for them.

Many interesting variations may be found in the legs and feet of flies, Lepidoptera, and other orders; but we must only further notice the hind-legs of the worker-bee. Here both the tibia and the first joint of the tarsus are broadened into wide plates, but the two sides of these plates are differently furnished. On one side they are rather hollowed out and comparatively bare; and in these hollows are carried home the pollen-

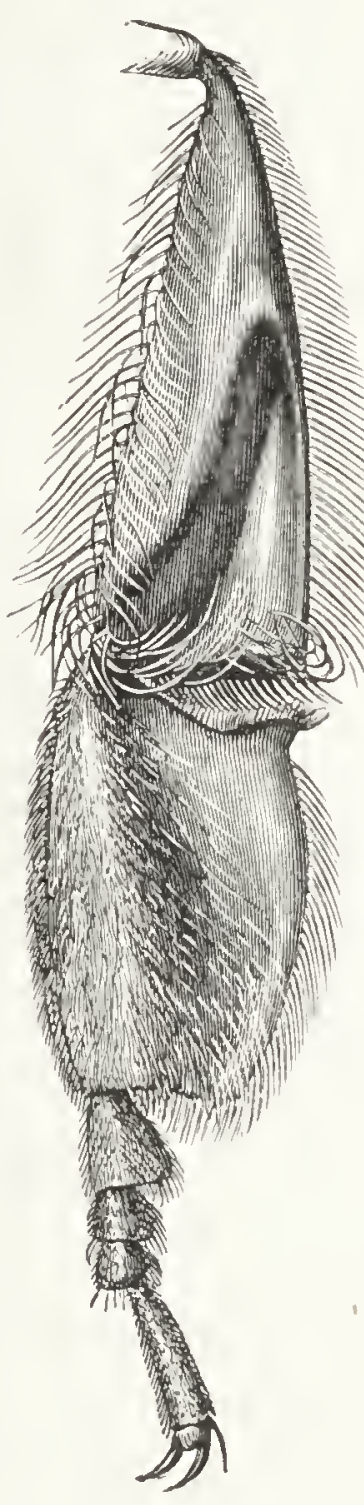

Ontside

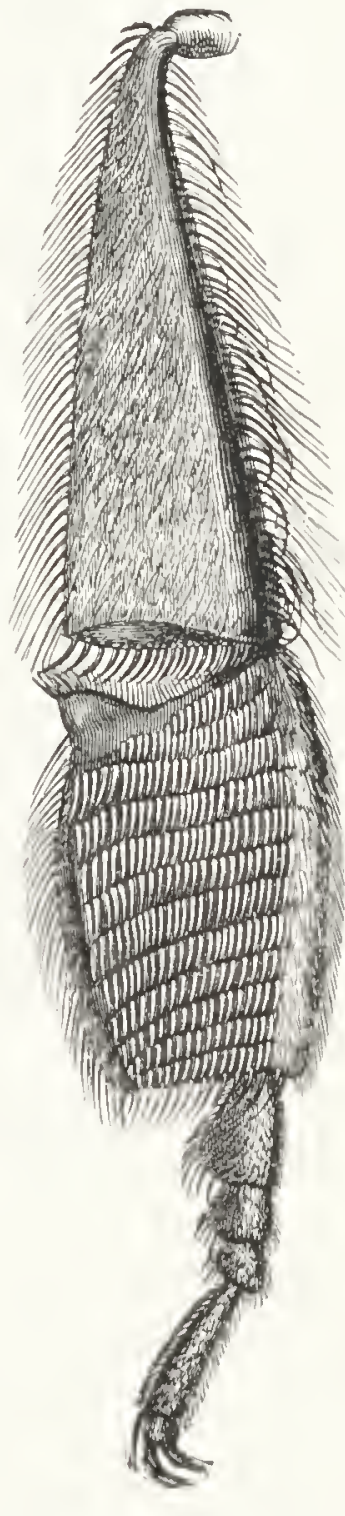

Inside

FIG. 129.-Leg of Worker Bee

bread and the resinous propolis used in finishing and cementing cells. On the other side are a thick coating of hairs, those on the tarsus taking the form of a brush, which appears to be used in collecting pollen. Neither of these special developments are found on the hind-legs of the drones or of the queens. 
So much for examples of quite different systems--but still systems-for the microscopical study of the insect world : we must content ourselves further with a few miscellaneous subjects of interest amongst insects and spiders.

96. Wings of Insects.--These offer very interesting and easy subjects for the 'comparative' method, all the more instruetive because upon them the great insect orders were originally, and are still mainly founded. The Orthoptera were the 'straight' wings, the Neuroptera the ' nerved' wings, the Trichoptera the 'hairy ' wings, the Coleoptera the 'cased' or 'sheathed' wings, the Diptera the 'two' wings, the

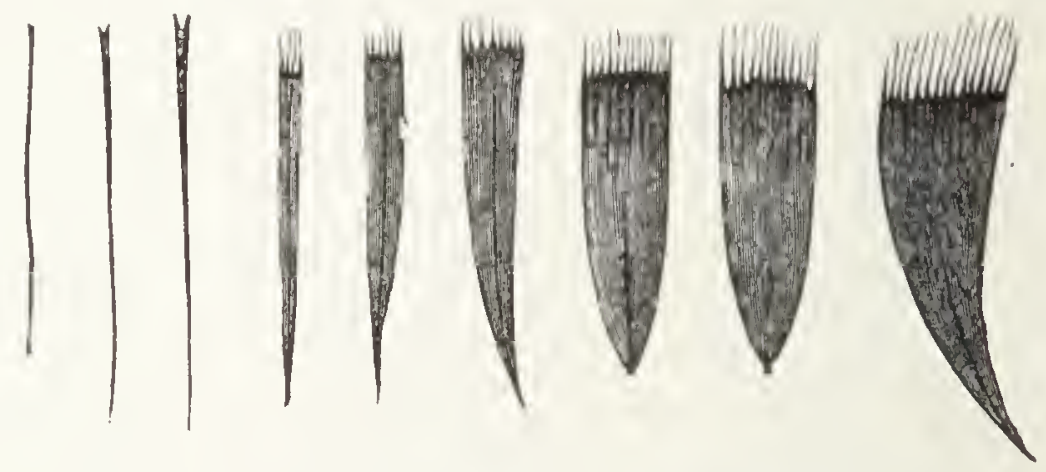

FIG. 130.--Scales from Convolvulus Hawk-moth ( × 20)

Hymenoptera the 'married' wings, the Lepidoptera the 'scaled' wings.

In all the orders there have been found most interesting variations, especially in such wings as are very minute. Some are best mounted dry: for instance the wing of the Hairy Midge (Psychoda) is apt to lose the hairs with which it is loaded if immersed in fluid-they come off so easily. And the smaller membranous wings are so transparent that some of them are really better as dry mounts. But the majority mount in benzol-balsam quite well. The thick horny cases of many beetle wings need to be made transparent by long soaking, in the manner already described (Chap. VII). The elytra or wing-cases of iridescent beetles make beautiful objects mounted opaque for the Liẹberkühn 
or reflector, and so do bits of wing from the iridescent and bright-coloured butterflies and moths.

Whole wings of the latter mount well in balsam. It is very easy to mount the whole or a piece of a wing to show the arrangement of the scales. Some wings will show the scales so sparse that they stand clearly apart, and in the

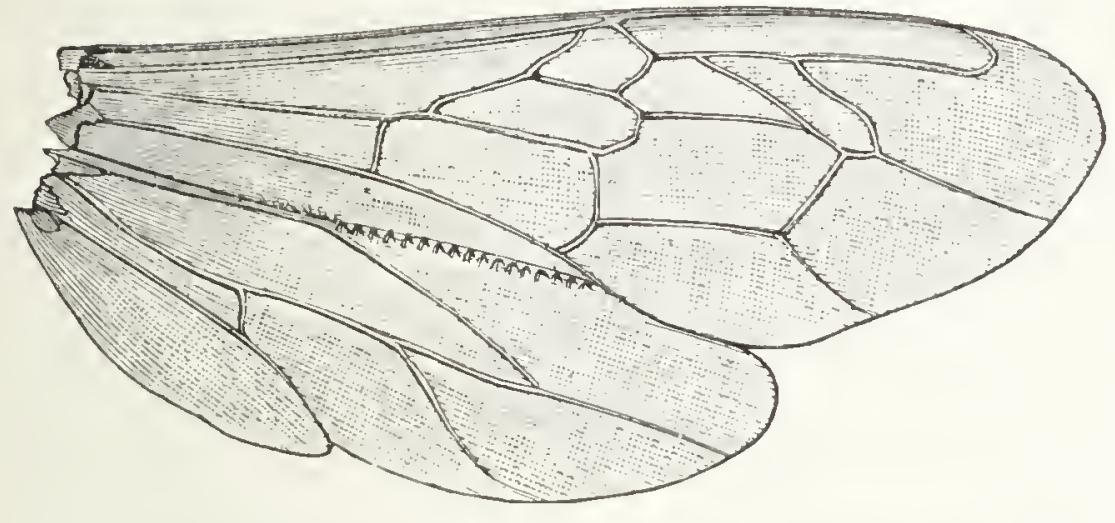

1 in flight

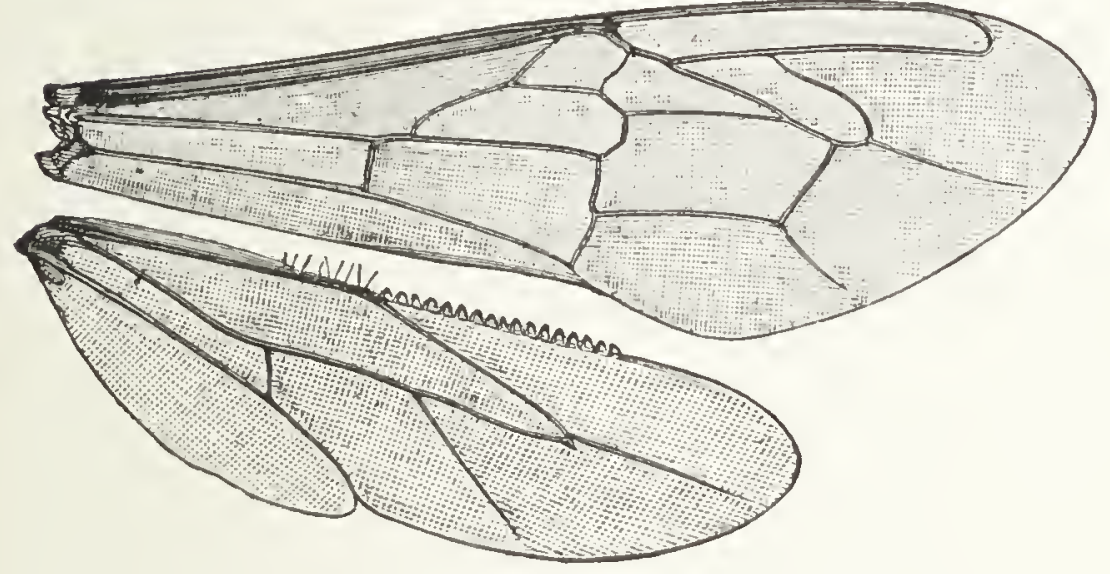

Separated

FIG. 131.-Wings of Bee $(x 7)$

clear-winged moths the scales are rare, and more or less interspersed with hairs.

A scale is in fact only a peculiar development of a hair, and the particular development varies amazingly. Scales rubbed off from different genera, and even species, and mounted dry, will make an interesting set of slides. Also from the Convolvulus Hawk-moth (and many others) sets of 
scales may be so selected and mounted as to illustrate clearly the gradual development of hairs into scales. Fig. 130 is drawn from a set of scales I had so mounted, all of which were taken from the same individual insect, and show the transition clearly. Again, it will be found that the membranous wings of all insects are covered with small hairs; and if the wing of a Gnat be carefully mounted, it will show that on many portions, especially the veins and the edges, these hairs have become scales, in no essential respect differing from butterfly scales.

Fig. 131 shows the Hymenopterous or 'married' type of wings. The two are also shown separated, to manifest on the hinder wing the row of little hooks standing up, and on the posterior edge of the fore-wing, the turned-down edge into which the hooks catch. Thus, when in position, the two are joined together. It will be found very interesting to trace this character running not only through the Bees and Wasps, but through such totally different-looking groups of insects as the Ichneumons, Saw-flies, Gall-flies, and Ants, even of small size.

97. Mouth Organs.-Similarly interesting and important are the mouth organs, equally important in classifying with the wings, and sometimes more so. For a primitive and normal type take the Cockroach (fig. 132). The main portions of the apparatus are here shown separated. With care the mouth-parts of insects can be so separated, and laid down upon the slide, arranging them in benzol-balsam, and leaving the preparation to nearly dry in the balsam, protected from dust, before laying down the cover-glass. Such a preparation is known as the trophi of an insect, and a set of trophi, of various species throughout the orders, is a most valuable and instructive set of slides, such as cannot be bought for money anywhere. ${ }^{1}$

1 A couple of dozen I possess, kindly mounted for me by the late $\mathrm{Mr}$. Tatem, of Reading, are among the most valued slides in my collection. 
Looking now at the cockroach's mouth, the whole apparatus is a complete upset of all our vertebrate notions, though in many respects similar to that of crustacea. There is a sort of roof or upper lip a called the labrum. Next come the first and principal pair of jaws $b$, the mandibles, but which work from side to side, as do all the organs, and not like ours. These are hard and strong and toothed. Below these are a second pair less strong, but toothed also, $c$, called the maxiltce, and each of which has a palp or feeler, but which is more really a sort of hand to help in manipulating things. Beneath all is the lower lip or labium, $e$, but which under higher power is made out to be essentially a third, but still

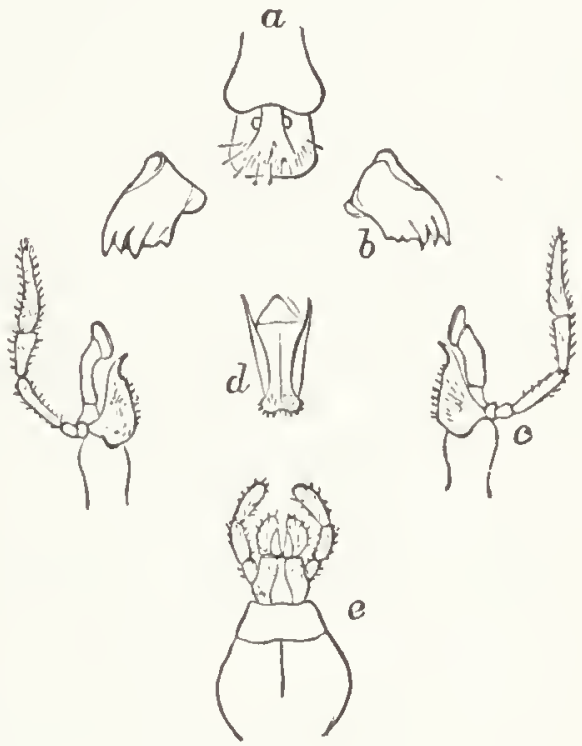

FIG. 132 less powerful pair of maxillæ, each of which also has a palp. In the cavity of the mouth is a lingua $d$, which is something like a real tongue, receiving the duct of the salivary glands.

Leaving this ancient type, it will be most interesting to see how in the main it is preserved in all the Beetles or Coleoptera, with chiefly the more or less complete disappearance of jaw-like character from the parts of the labium, or third pair. Next to be modified are the second pair, the maxill:e. It will be found of great interest to follow up, with the help of some really intelligent work on entomology, the transformations of these organs and their palpi, with those of the labium, into the 'tongue' of bees and wasps, the 'proboscis' of moths and butterflies, and so on ; the mandibles either remaining as biting jaws-as in the bee-or being reduced to rudiments, as in butterflies. We take two examples from a different order again, the Diptera, in which these organs 


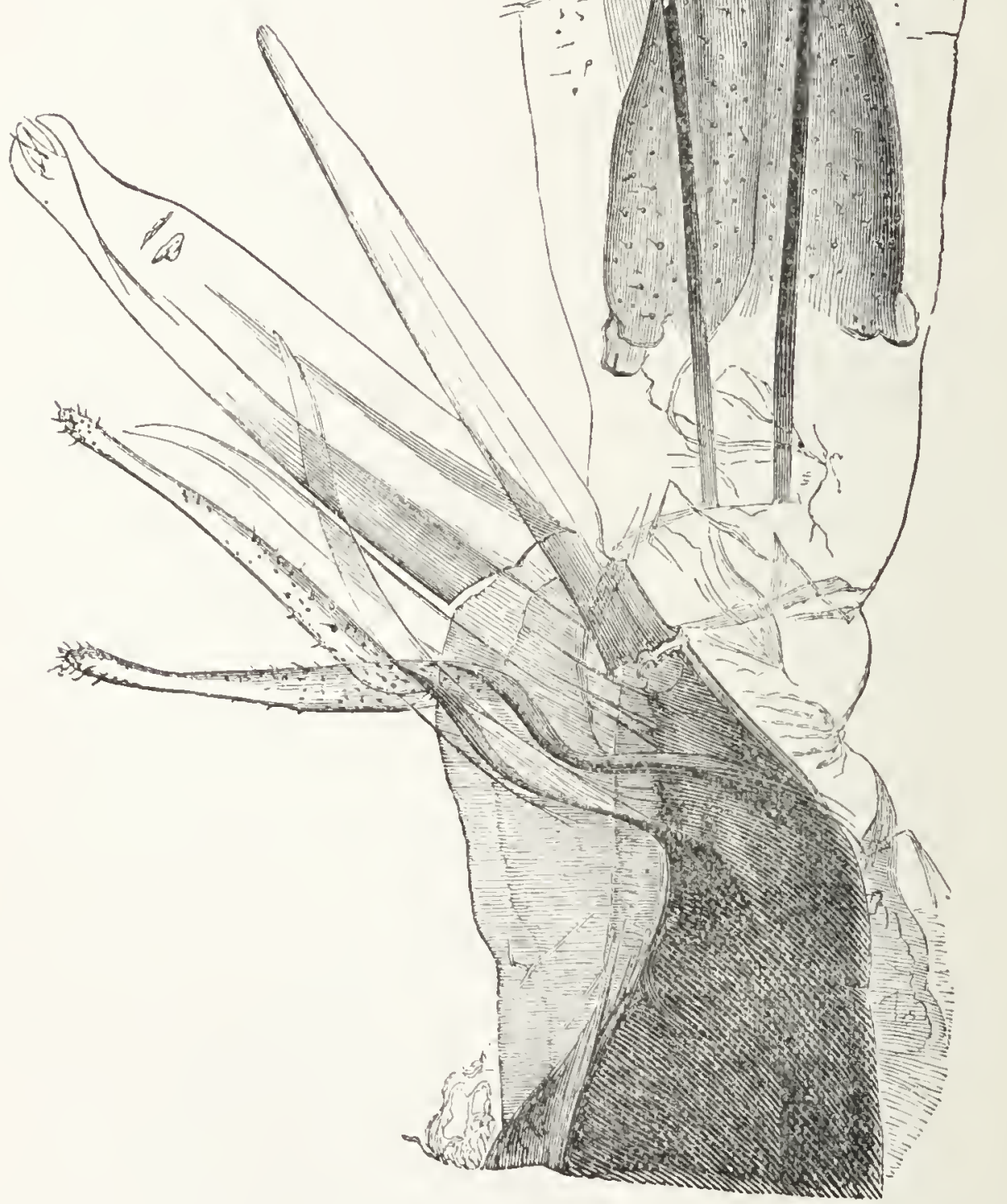

FlG. 133.-Mouth Organs of Drone Fly ( × 25) 
are so modified as to assume more or less of the character of weapons. Fig. 133 shows those of the Drone-fly, taken first as resembling in many respects those of the common fly already mentioned (fig. 117). The soft parts of the proboscis, with their pseudo-tracheal tubes, are almost precisely similar, as will be seen, and represent the anterior part of the labium. The labrum, mandibles, and maxillre are in the Diptera converted into lancet-shaped organs more or less developed, which both pierce the skin of animals, and form tubes by which the juices may be sucked

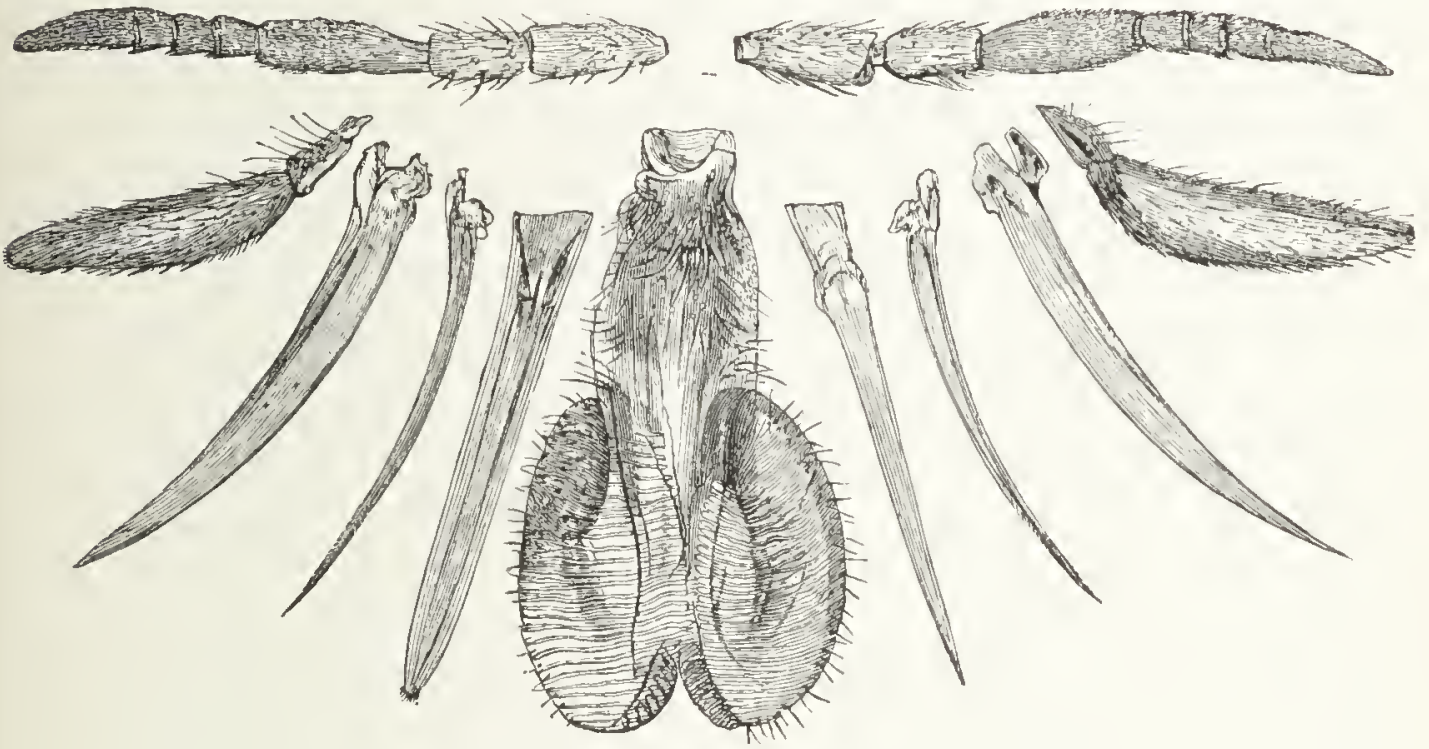

FIG. 134.-Trophi of Tabanus

up. In the Drone-fly they are much larger than in the House-fly, and easily made visible. The figure shows clearly the maxillary palpi, next which come a couple of lancets, and finally two instruments resembling by comparison straight two-edged swords, and a very peculiar one with prongs or teeth at the end, the purpose of which appears to be to enlarge and irritate a wound and so increase the flow of fluid, which is probably sucked through a tube up the centre.

The further development of this type of mouth is well seen in the organs of a Tabanus or Breeze-fly, fig. 134. These 
are here shown laid out separately, with the antenna added, as a specimen of the mounted 'trophi' already recommended. The general strong resemblance in the several organs is easily seen at a glance, and also that the line of development is carried still further, the fleshy ligula or proboscis being diminished, while the offensive organs are increased in power, till they appear like half a dozen different instruments from a cutler's shop.

In Bees the mandibles remain, and both pairs of palpi, but other organs are modified into the tubular proboscis, so well known. In Lepidoptera the mandibles are mere rudiments, and the proboscis is immensely long, and kept when not in use curled up into a spiral. It is really in two halves, the tube being made up of two half-tubes or gutters with their open diameters in contact.

98. Antennæ.-Many of these organs are of exceeding beauty, especially the feathered or plumed ones of many Lepidoptera and of the Gnat family. They differ to an altogether extraordinary extent in different orders, and even different genera. They appear in very many orders to have most to do with the mysterious power the males of many insects possess, of finding the females; so that a crowd of some moths will gather round an unpaired female, even when concealed in a pill-box about one's person. At all events, in a large number of cases, as the common Cockchafer-the antennx are immensely more developed in the male; and when this is the case it is well to mount both sexes upon one slide.

Eyes have been sufficiently dealt with on p. 180. Such exquisite sections can be obtained professionally mounted, that it is a pity not to avail oneself of results which can hardly be rivalled by any amateur.

99. Ovipositors. - The ovipositors of female insects offer immense variety in the different orders. In Crickets and Locusts they are very large indeed. The most interesting 
are perhaps those of the Hymenoptera. In Ichneumons they are mostly long and pointed. In some genera they become boring organs, as in the great Sirex gigas. In Bees and Wasps they are connected with poison reservoirs and become stings, which are accordingly confined to the female sex entirely, the males being helpless. More or less serration at the tips belongs to nearly all. In Bees and Wasps the teeth are barbs; but the most interesting

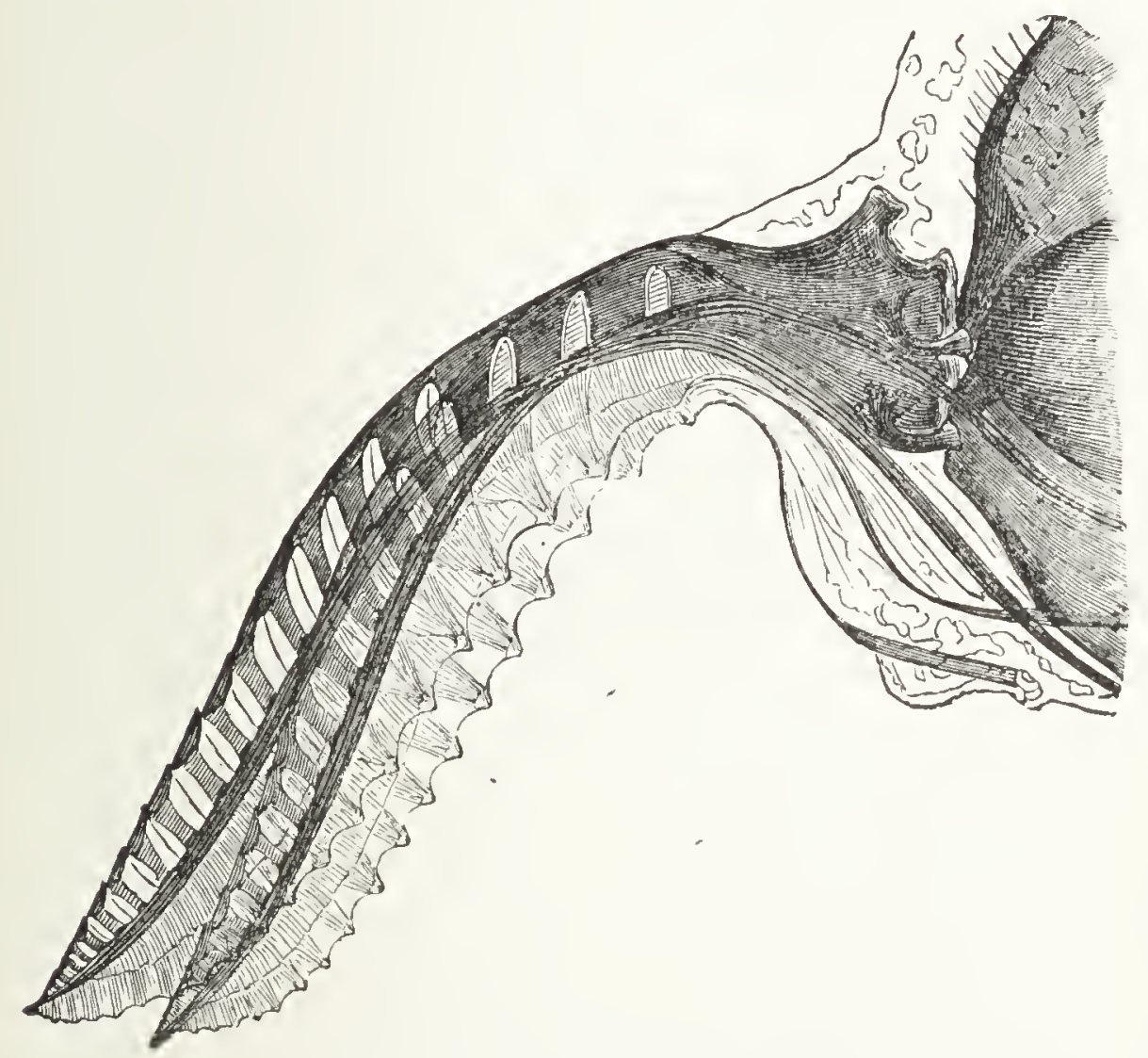

FIG. 135.- Saws of Saw-tly $(\times 40)$

development of this toothed feature is in the Saw-flies, of which there are many genera. The saws of a very common fly are shown in fig. 135. In every case the saws are in pairs, and each 'blade' or cutting-saw works backward and forward in a grooved sheath which embraces its back edge. The two saws work side by side with alternate motions, and thus cut a groove in the leaf, or rind, in which the eggs are to be inserted. The exact 
pattern of teeth differs somewhat; but a very curious fact is that most of them, as can be seen from the figure, almost exactly resemble a pattern which has been adopted in some surgical saws, and some saws made in America for various purposes.

100. Eggs.-Ovipositors naturally remind us of the eggs of insects. Many of these are very beautiful objects. Fig. 136 shows some eggs of butterflies magnified, while the upper right-hand figure illustrates the natural size, and the manner in which they are generally deposited. This how-

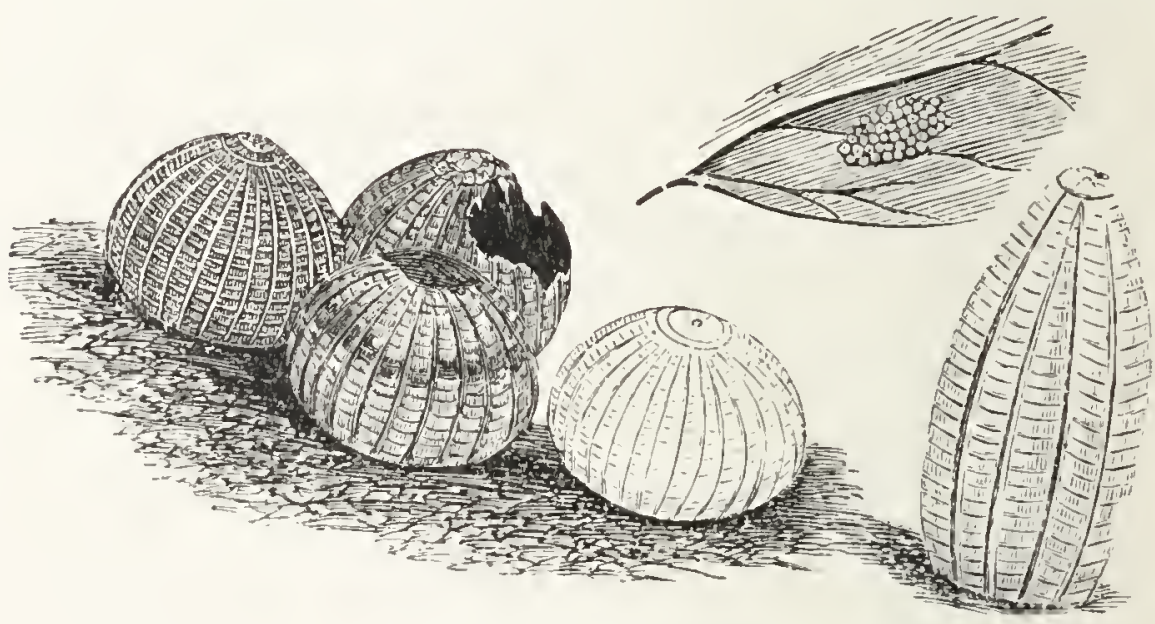

FIG. 136.-Butterfly Eggs

ever differs a great deal; some being attached to a kind of stalk, and others laid in coils or spirals round small twigs. They are best mounted 'dry' upon a black ground.

Still more striking are the eggs laid amongst the feathers of birds by the numerous parasitic insects which infest them - each genus of birds, as a rule, having its own parasite, as each genus of animals has its own flea. Fig. 137 reproduces by permission a beautiful drawing by Dr. Dallinger of three of these eggs. That numbered 1 is the egg of a parasite of the Peacock; No. 2 that of a parasite on the Hornbill; and No. 3 is from the Australian Mallee-bird.

- But analogous forms are to be found in the plumage of our home birds, game, and even of domestic poultry. 
101. Spiders.-Various points in these wonderful creatures are worthy of microscopical demonstration. Their eyes are single instead of compound, and usually eight in number, arranged as an upper and lower row of four each. By skilful section-cutters, thin sections of these are prepared, showing the lens, and the rods of the retina. The head and

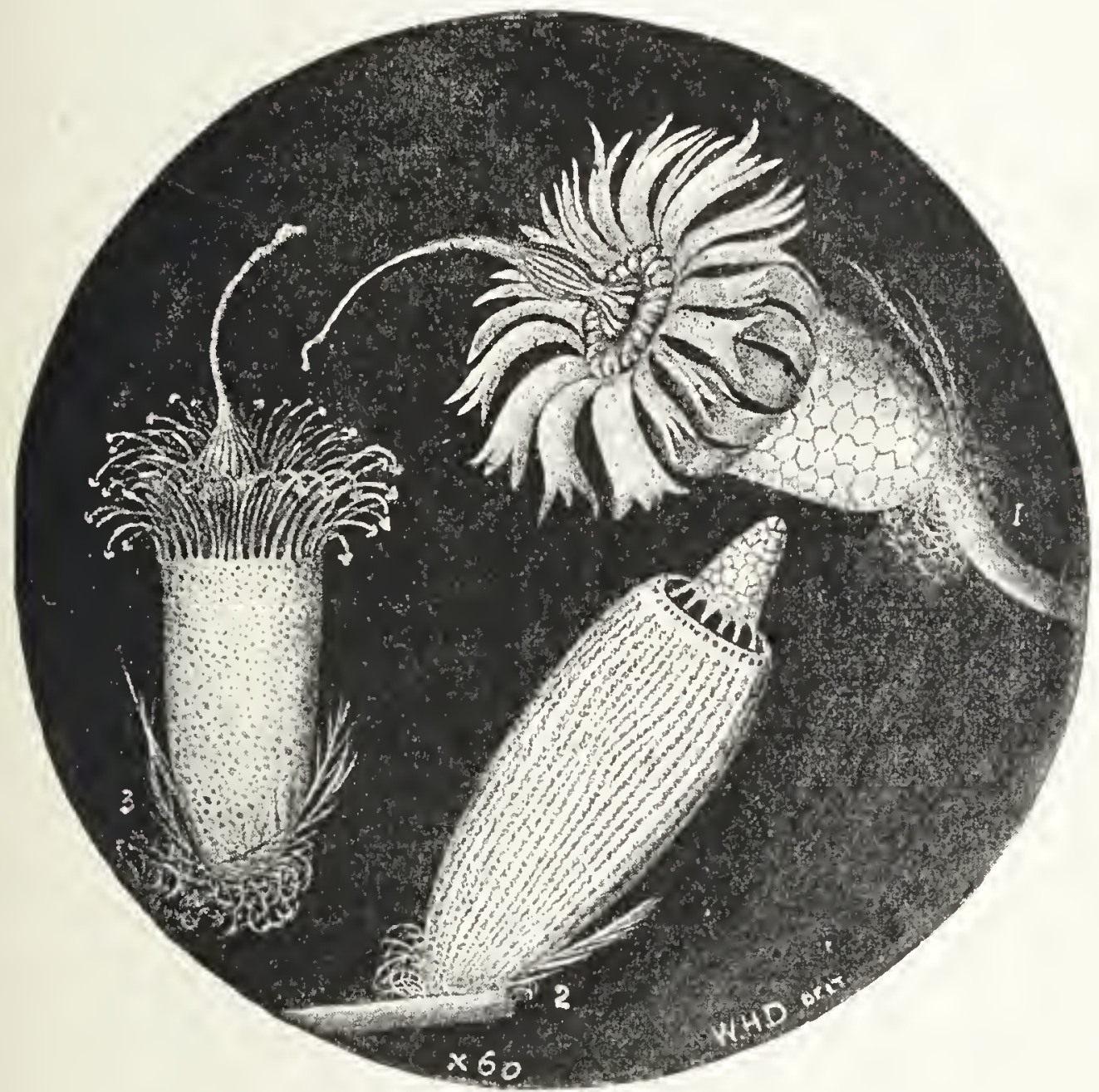

Fig. 137.-Eggs of Bird Parasites

jaws are easily mounted, and convey a vivid idea of the terrible offensive power of these creatures in proportion to their size. Imagine a cat with a pair of claw-jaws the size of those of a large crab, and with poison in the hollow fangs: that is the scale on which a spider is armed. But perhaps most general interest attaches to the organs associated with the web and its formation. 
Fig. 138 shows the cloaca and spinnerets of the Garden or Geometrical Spider. The Spinnerets at a first glance appear four, but are soon seen to be six in number. They are not single teats or tubes, but contain in some species up to a thousand separate tubules. The microscope itself will make apparent, that the lower pair contains tubes much larger than the others. But it has required attentive observation to discover, that these large tubes emit a more liquid and viscid secretion than the others, and that the spider has the power of ejecting filaments from the hundreds of pores at once, either so that they remain

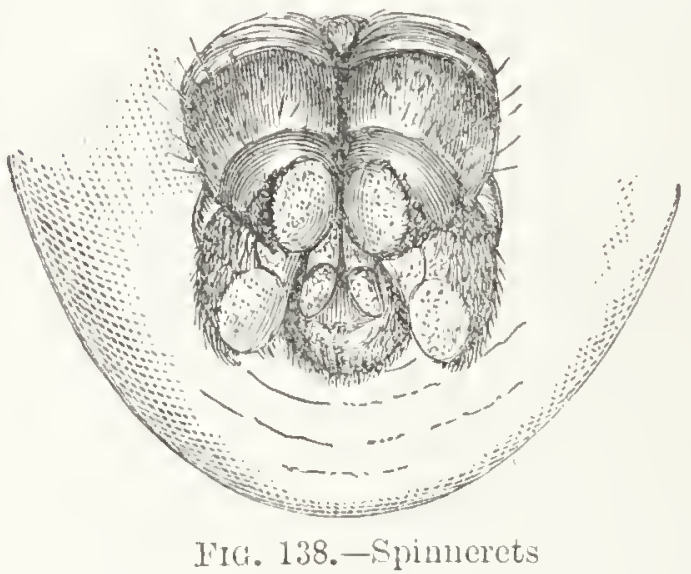

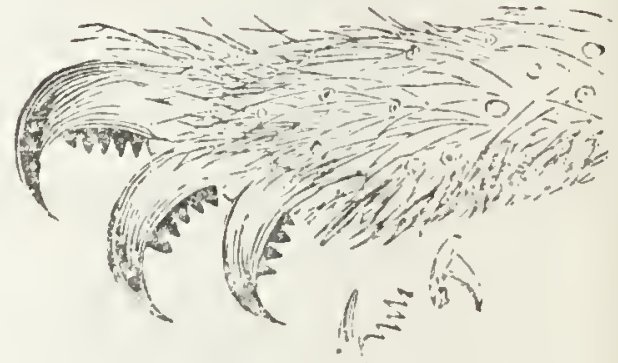

FIG. 139.-Foot of Garlen Siniter

separate threads, or so as to unite almost immediately into one stronger thread. She spreads them when making the holding end, or anchorage of a line, before it unites into one; also she spreads them when winding up a victim in a shroud of silk, turning it over and over with her legs whilst emitting the tangle of silky filaments. In ordinary web-spinning she converges them, so as to join into one cable almost at once. Statements about her 'handspinning' are fables.

Yet the foot has to be in relation to the lines, upon which the insect is constantly running with the greatest rapidity. It is terminated by claws armed with many comb-like teeth. The great claws may be two, but in many garden sorts are three, with smaller claws which can oppose them as well- 
the latter are shown separate in the figure. However quick the pace, the line falls between some pair of the teeth in the comb, and the foothold is sure and steady.

The radii of the web are spun with ordinary silk (which can in fact be gathered and spun as silk, were there enough to be worth while) which is not at all adhesive. By these the spider runs from the centre to her prey. She spins these first, after her marginal foundations; and next spins a continuous Archimedian spiral thread from centre to circumference, of the same non-adhesive thread. She then goes back from the circumference to the centre with another spiral; but this time the large spinning tubes are also brought into play, and the hard thread as it is spun is

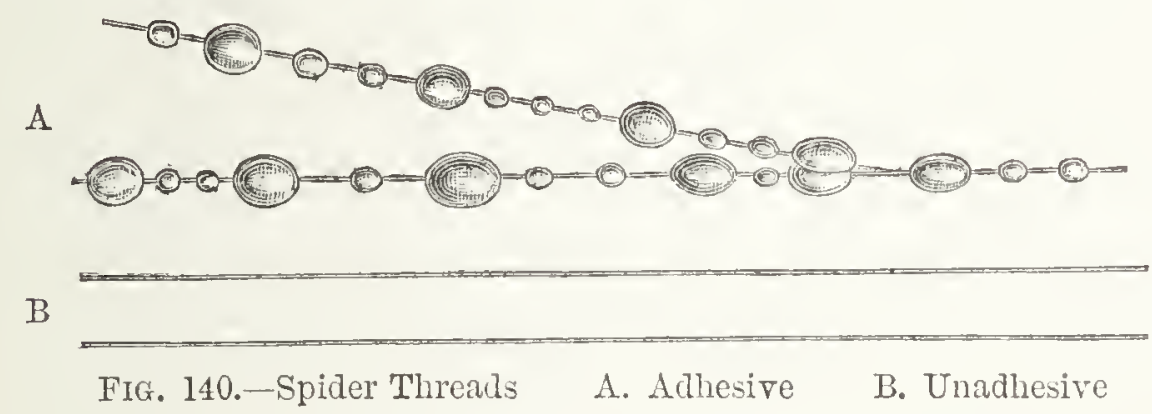

covered with viscid non-drying fluid, which appears chiefly as globules strung upon the thread. Larger globules at pretty regular intervals, have generally very tiny ones interspersed all along the intervals, as in fig. 140. It is these globules which detain the flies. ${ }^{1}$ They adhere to the spider' also, if her foot touches a globule, contrary to the common belief. She constantly has to tear away rungs from her ladders to get free of such mishaps, and as constantly repairs them: and in some cases at least, unless a fresh web is made altogether, which is often done, the adhesive lines have to be renewed next day, the fluid probably drying.

1 It is often stated that in spinming the viscid set the spider cuts off and eats the unadhesive spiral, which is used as a bridge only. Without affirming that this never occurs, I am absolutely certain that it was not done in cases which were watched. It may be that species differ in that point. 
Much has been written, in ignorance of the true state of the case, about the wonderful skill of the spider, and her dexterity, in forming such a number of these viscid globules so rapidly. If it be remembered that there are hardly ever less than 100,000, and often a quarter of a million, in a web spun in less than an hour, it will be seen that such dexterity is impossible. The truth is far more really wonderful, than any such mere marvel would be (if physically possible). Years ago the late Mr. R. Beck observed that these threads when first spun were quite smooth, but that first undulations and then the globules rapidly appeared. But only quite lately has Professor C. Vernon Boys, F.R.S., shown clearly that if a fine quartz fibre be stroked with a straw dipped in castoroil, the fluid in a few moments forms undulations and then drops, in just the same way; nay, these drops of castor-oil are equally effective in catching flies. This, then, is what happens. The tough thread, as fast as it is spun, is coated with the fluid; and the web-spider's mode of subsistence, and her complicated spinning organs, are bound up with, and work in association with, those profound molecular laws which cause a jet of water to break into drops, or hold together a soap-bubble!

102. Life Histories.-Only one more line of work can be indicated here. There are many cases, especially where very minute insects are concerned, in which the use of organs, or the complete life-history, is yet unknown. Three illustrations must suffice.

More than thirty years ago (1863) Sir John Lubbock discovered that a minute hymenopterous insect (called by him Polynema natans) descended into water, both swimming and crawling therein. Since then the insect is only known to have been observed three times until. quite lately (May 4, 1895), when it was rediscovered near London by $\mathrm{Mr}$. W. Burton and Mr. F. Enock, and kept under observation. The first result was the discovery of the unknown fact, that the 
specimen observed never left the water during several days! Possibly its history may be completed by these observers; but when it is added that some allied species only measure $\frac{1}{75}$ th inch long, and that there are other insects quite as small (the Trichopterigia among beetles being only $\frac{1}{30}$ th to $\frac{1}{30}$ th inch) it will be seen what lines of inquiry amongst the more minute insects await the microscopist.

There is another class of histories, in those insects which present the phenomena of alternate generation. Thus, the Aphides will go on for generations producing only females, which bud off as if were, asexually; while late in the autumn true males and females appear, which lay eggs in some sheltered place, thereby preserving the species through the winter till next spring. But M. Lichtenstein discovered that some Aphides presented more complex phenomena, scveral different forms appearing in succession before the final true males and females appeared. It has not been determined yet how far this complicated system of generations is common to the family; but it is pretty certain that there are many Aphides whose full history is unknown.

Our last illustration is from the Gall Flies, and that member of the Cynips family which produces oak-apples will do as well as any. Everybody knows the oak-apple, which is a large gall formed by irritation of the tissues in the twigs of oak-trees, by maggots hatched from eggs laid therein by flies of the Hymenopterous order. On the gall the maggot feeds, changes to a pupa, and hatches into a hymenopterous fly. All this seems like the ordinary development of an insect. But it is only half the story. The flies thus developed do not deposit eggs on the twigs again, but on the roots, where each gall is only as large as a pea; many galls being however often fused into one mass. The galls are also this time reddis $h$, and not golden-brown as the oak-apples were. These galls are formed in autumn, and the larvæ hatch in winter, and are pretty similar to the others. But the perfect 
insects are so widely different, that till quite recently they were classed in different gener $\alpha$. They are all without wings, and all females only, which lay their eggs in embryo buds on the twigs; these eggs forming oak-apples in turn. These facts were discovered by Dr. Adler; and since then many other double forms of galls and insects have been discovered, in a few of which the second form develops both sexes, though of different forms to the first. In nearly all these cases the two forms were formerly described as distinct genera, and the eggs are laid and galls formed in different situations, and are of totally different appearance.

There is little doubt that patience and research will discover other cases of these double forms, and perhaps associate into one descent other insects now considered distinct. But we have said enough to indicate more than one line of work calculated to afford the greatest interest, in the wonderful Insect World.

\section{CHAPTER X}

\section{THE VEGETABLE WORLD}

UNDER this heading, again, we can only briefly indicate some directions in which the microscopist may find interesting and profitable occupation.

103. Cells. - Thin slices of the cuticle of leaves, or of seeds, and sections in various directions of any tissues, or hairs from any plant, removed and placed on a slide under a cover-glass with a drop of water, will soon give a general idea of the numerous cells of which any highly-organised plant is composed, and of their great variety in form and character. The tissues of the whole plant are at once seen, 
far more easily than in the case of animals, to be built up of many single cells.

104. Single-celled Plants.-If the student possesses already a little skill, and a really excellent $\frac{1}{6}$ th which will bear a high-power ocular, a small particle of yeast will enable him to study cell-growth and multiplication in a very simple form: but this plant is too minute and transparent for beginners.

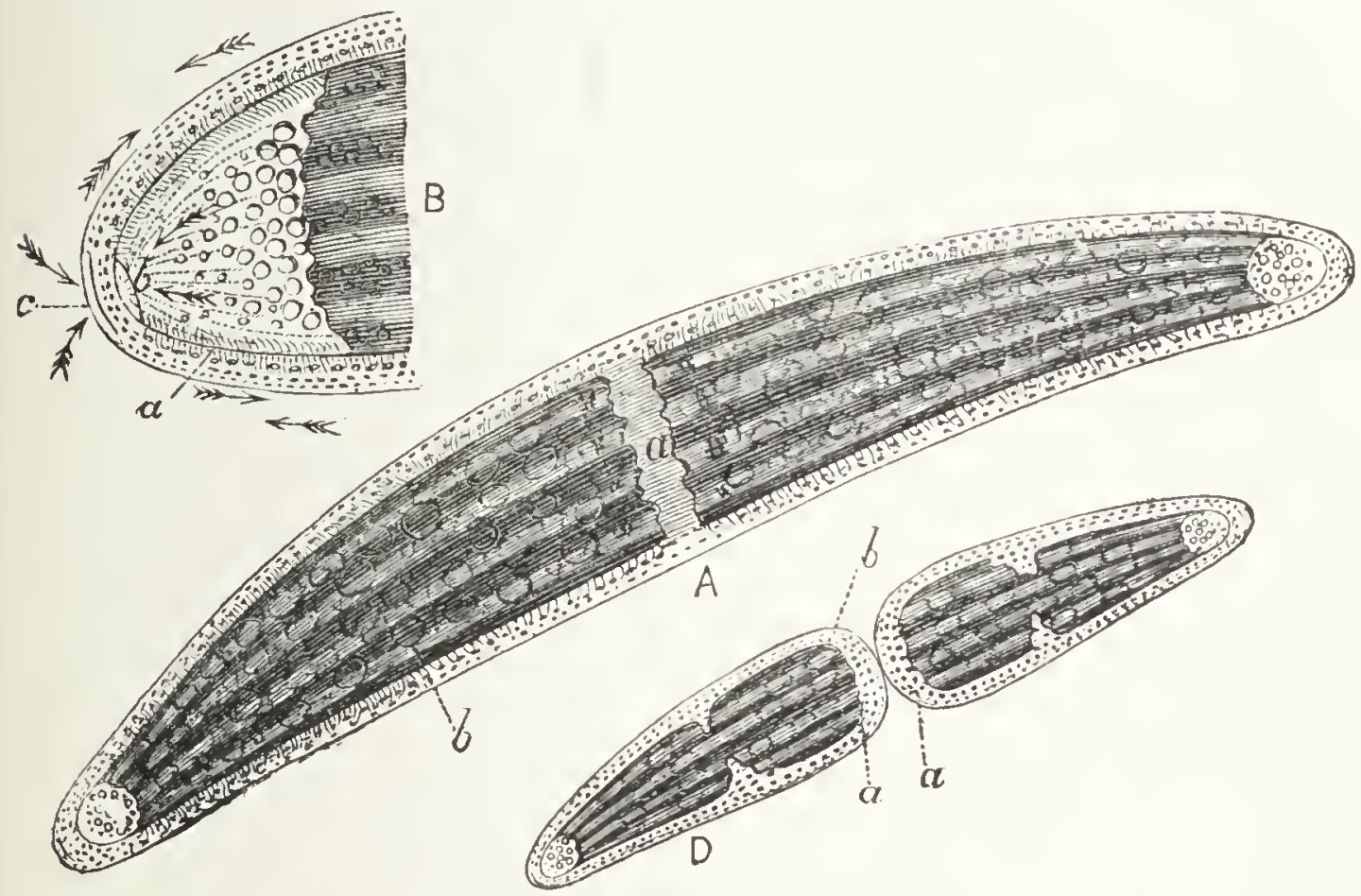

Fig. 141.-Closterium lunula
A. Perfect cell
B. One end enlarged to show cyclosis
D. Cell just divided

Bacteria are not treated here for the same reason. The serious student will have recourse to one of the many special treatises, and will require a good $\frac{1}{6}$ and an immersion. The ordinary ones can be well seen with a good $\frac{1}{5}$ or $\frac{1}{6}$ and compensating ocular, and slides can always be procured at $2 s$. or $3 s$. each.

Desmids grow in quantities in most ponds, especially those in which the water looks green; that colour being usually given by swarms of these plants, on which many aquatic creatures feed. Some may be got in the net: others require to be 
scraped off the bottom, or from the stems of submerged plants. They are of great variety in form, some of which are very beautiful: we can take but one example, from the group of Closteria (fig. 141). This is a very common Desmid, and shows clearly the main phenomena of the family.

First of all, it is easily seen in this Desmid that the living cell is full of living protoplasm, whose constant motion in streams presents the phenomena of cyclosis (\$114). The stream of particles is most clearly seen along the edges, but with care can be made out anywhere under the enclosing membrane. Every now and then it carries along with it one of the larger particles (marked $b$ in $A$ ) into the chamber at the end. These larger particles are not found in the central dividing space $(a)$ between the two halves of the Desmid; which leads to the inference that the cell in this state (A) is already partially divided into two. At $\mathrm{B}$ the end is shown much more enlarged. It is then seen that the inner membrane or endochrome is furnished with citia $(\mathrm{B}, a)$ which are in motion. The protoplasm outside the endochrome shows currents in opposite directions, as denoted by the arrows : in the end chamber are also very strong currents shown by the arrows converging towards $b$, and these currents appear thrown back or reflected as by the arrows pointing to $c$. The whole gives a very vivid idea of the active life going on in the cell.

At $\mathrm{D}$ is shown the simplest form of propagation, by celldivision. The endochrome retreats more and more from the central space $a$ in diagram $\mathrm{A}$, while there is outer constriction at the same time, till the two separate entirely as at $a d$ in diagram $\mathrm{D}$, the exterior becoming rounded as at $b$. The exterior ends $b$ then elongate, while each half begins to constrict in the middle; and in about six hours each half has become symmetrical or nearly so, like the parent.

This and other Desmids present also at intervals the phenomenon of conjugation; two individuals uniting, 
and producing a zygospore which forms a fresh series. Some good fortune as well as patience will be required to secure specimens in the stage of conjugation. This simple conjugation of two cells apparently precisely alike (though it cannot be proved that it is really indifferent what cells enter into union; there may be differences, of the nature of sex, which the microscope cannot discover) is evidently the earliest steps towards the truly sexual mode of propagation.

105. Algæ.-Almost every pond open to the sun will furnish a variety of these plants, mostly in the shape of long green filaments, which only need to be placed upon a slip with a drop of water and a cover-glass. In these filamentous forms we have a number of similar cells joined together end to end, the simplest form of a complex structure.

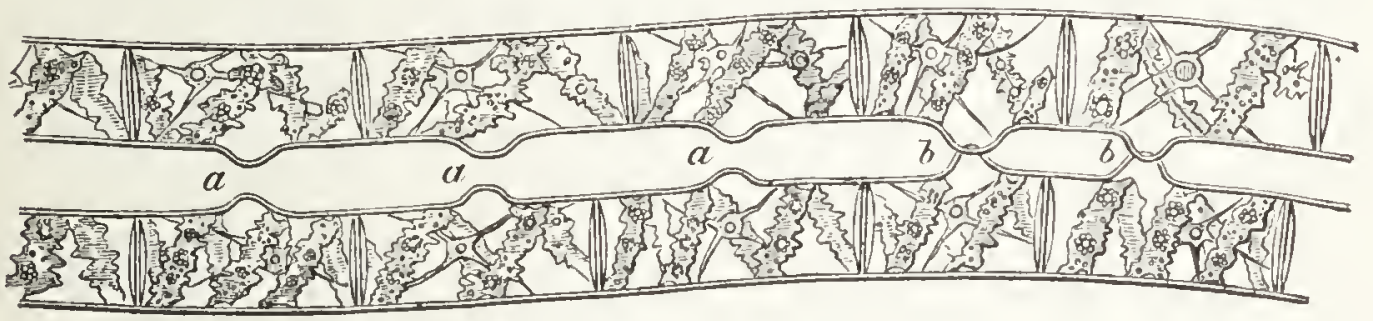

FIG. 142.-Spirogyra conjugating (Sachs)

Many of the plants are of great beauty when seen on the stage, especially those of the Spirogyra family. The cells of this group for a while divide in the middle, each half lengthening so as to extend the filament; and during this period the endochrome and chlorophyll bodies usually remain a compact mass. But as the cells mature, the endochrome contracts, and shapes itself into a beautiful spiral green band inside the outer tube, from which the family takes its name.

The Spirogyra furnishes another example of conjugation between two cells similar to all appearance: but there is a distinct further advance in the process. Two adjacent filaments whose cells are mature, throw out from each cell lateral protuberances (fig. 142, a). These grow till they 
meet (b), and meantime the spiral contents of each cell shrink and gather into an ellipsoidal mass. Then the cell wall opens between the two projecting parts, and one of the two ellipsoidal masses passes gradually through to coalesce with the other. Close observation, however, shows that the spiral band is preserved, though forced together into a

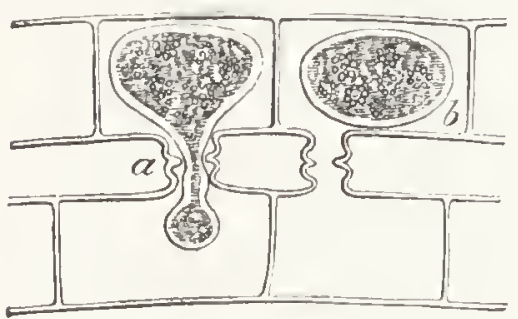

FIG. 143.

Conjugation complete (Sachs) compact body; and moreover after coalescence the two bands are so arranged as to form one band. The passage of one mass into the other is shown nearly complete at $a$ in fig. 143, and quite so at $b$. The 'zygospore' thus formed remains apparently quiescent for some time, often months; and then begins to elongate and divide to form a new filament.

It is to be remembered that, while the two cells show no observable difference, the contents of one are cntircly absorbed by the other : also that all the cells in one filament, are usually absorbed by the cells in the other. These facts appear to indicate that there is a difference in the two cells, though it eludes present observation; and that we have here a further advance towards difference and union of sexes.

106. Alternation of Generations.-We further have in the above an early stage of the phenomena called Alternation of Generations. Just as in the Aphides (p. 199) we had generations budding from imperfect females only, to be succeeded at intervals by true union of sexes; so here in the vegetable world, we have multiplication for a period by division and growth of cells only, but followed after a while by union of two individuals. These so far appear alike; and yet we have indications that already they are in some way different. But in some form or other, Alternation of Generations between some form of cell-multiplication, and the formation of new individuals by conjugation of two, prevails through the entire vegetable world. The details of Fern propagation 
are very remarkable, but must be studied in botanical works : in this class of plants alternation is regular. Potatoes have been propagated almost entirely for generations by 'eyes' or tuber-buds. In mosses, it is the asexual product usually observed. The Chara family form at intervals perfect fruits; but there is one species-C. crinita-in which only female organs have been found for many generations in most Continental localities, though a very few male organs have now and then been observed in Transylvania. There are many various phenomena of this kind, which are not fully understood, and will furnish subjects of profoundly interesting microscopic research to the botanical student.

107. A Life History.-As just one illustration, let us take the history of the common mildew or rust in wheat, which has only lately been established by the researches of De Bary, and which may be compared with that of the Gall Fly in $\$ 102$. As in that case, investigation has shown that what were once considered fungi of different genera, are but stages in the history of one. There are found in spring on the leaves of the common Barberry orange-coloured swollen spots,

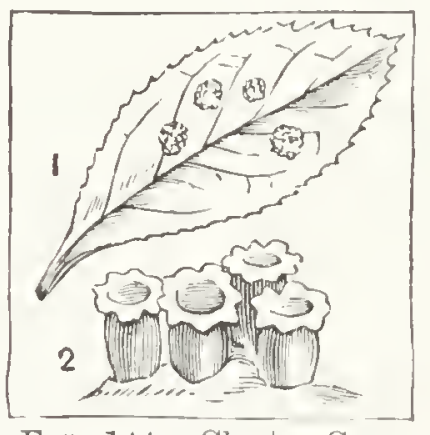

F[G. 144.-Cluster Cups

1. Nat. size

2. Magnified which under a powerful hand-magnifier present the appearance of clusters of small cups, as in fig. 144. On making vertical sections, the swollen parts are found to be full of the thread-like mycelium of a Fungus. There appear however to be two forms of fruits, which themselves were once considered two fungi, but are now known to be but two forms of one, as shown in fig. 145. Here $x$ is the natural thickness of the leaf, which is so swollen by the thread-like mycelium. From the mass protrude on one side the 'spermogonia' sp, urn-like receptacles lined with mycelium, whose threads protrude through the epidermis. From these receptacles are detached minute bodies called spermatia. 
The other form of fruit is much larger, and is the old AEcidium of botanists; a name still used, but only as a stage in the history of the mildew Puccinia graminis.

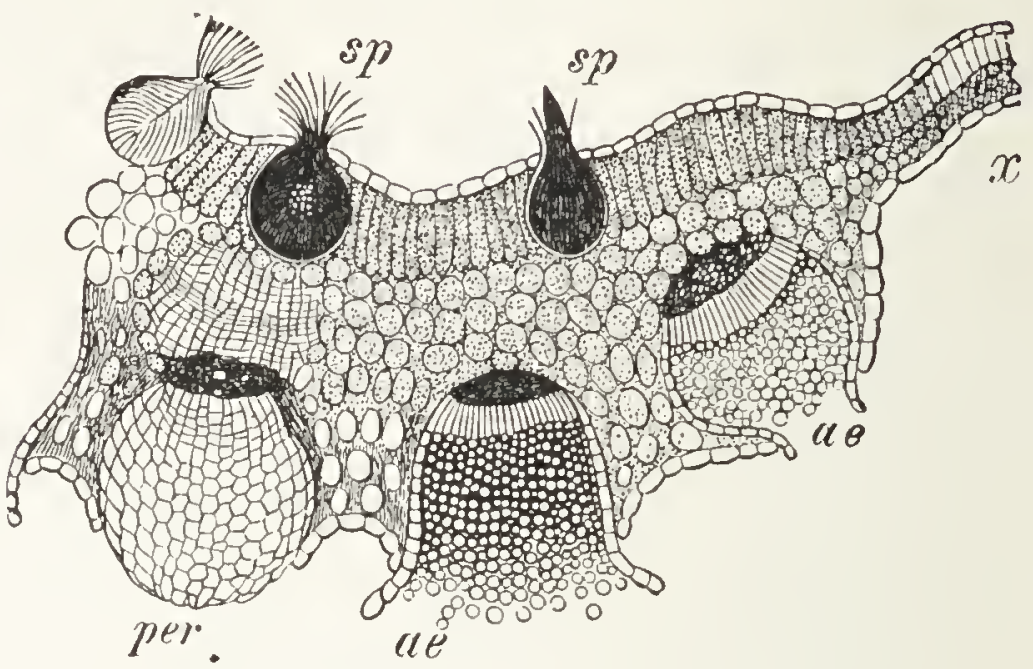

FiG. 145.-Section of Barberry Leaf (Sachs)

These also spring from the mass of mycelium, and at first are nearly globular bodies per beneath the cuticle, lined with a layer of cells called the peridium. These finally

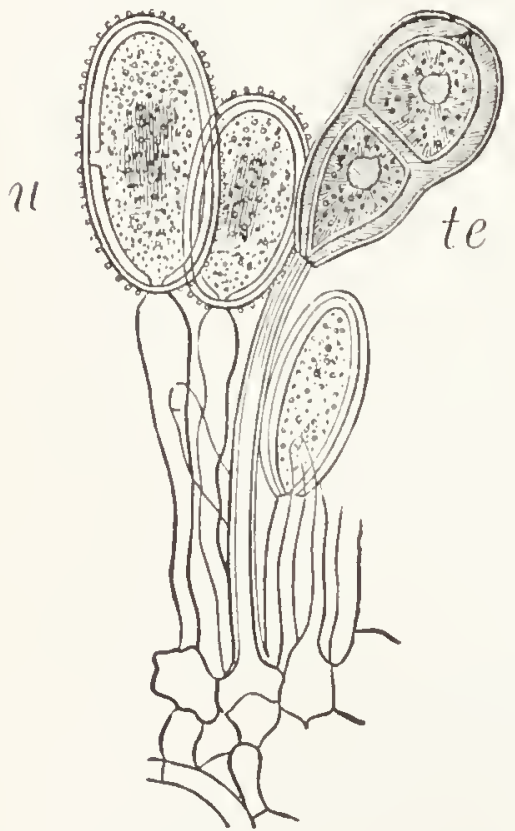

Fig. 146.

Uredo-spores (De Bary) break through to form the open æcidium cups ae, and which give off chains of larger æcidium spores. As will be seen, a section showing these stages of development makes a very beautiful microscopic object.

These spores (whose union in some way with the spermatia is probable, but has not even yet been proved) cannot germinate upon the Barberry. But on the stem or leaf of wheat or grass, they throw out germinating filaments which enter the pores or stomata ( $(113)$ and form another network of mycelium there. This mycelium produces another form of spore or fruit known as uredo-spores or fruits, and shown at $u$ in fig. 146, which 
are borne at the ends of filaments, and are known as the ' mildew' on the wheat. These uredo-spores germinate on the same grass or wheat, and the mycelium from them forms similar spores, this going on for several generations. At last the older uredo-plants begin to produce amongst the others, at first single specimens te (fig. 146) of another and larger two-celled kind of spore, known as teleutospores. Then the uredo-spores cease, and the uredo-plant produces only groups of teleutospores, as in fig. 147, which break

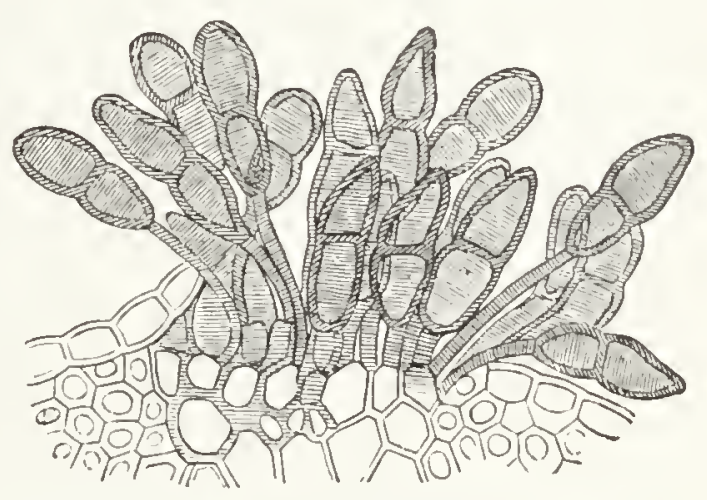

FIG. 147.-Teleutospores (De Bary) through the cuticle of the wheat-plants. These teleutospores remain on the haulms through the winter, and in the spring they germinate and throw out filaments, at the ends of which yet another kind of sporidia are produced. These germinate and produce mycelium only when they find a lodgment on the surface of the leaves of the Barberry, with which event the cycle commences over again.
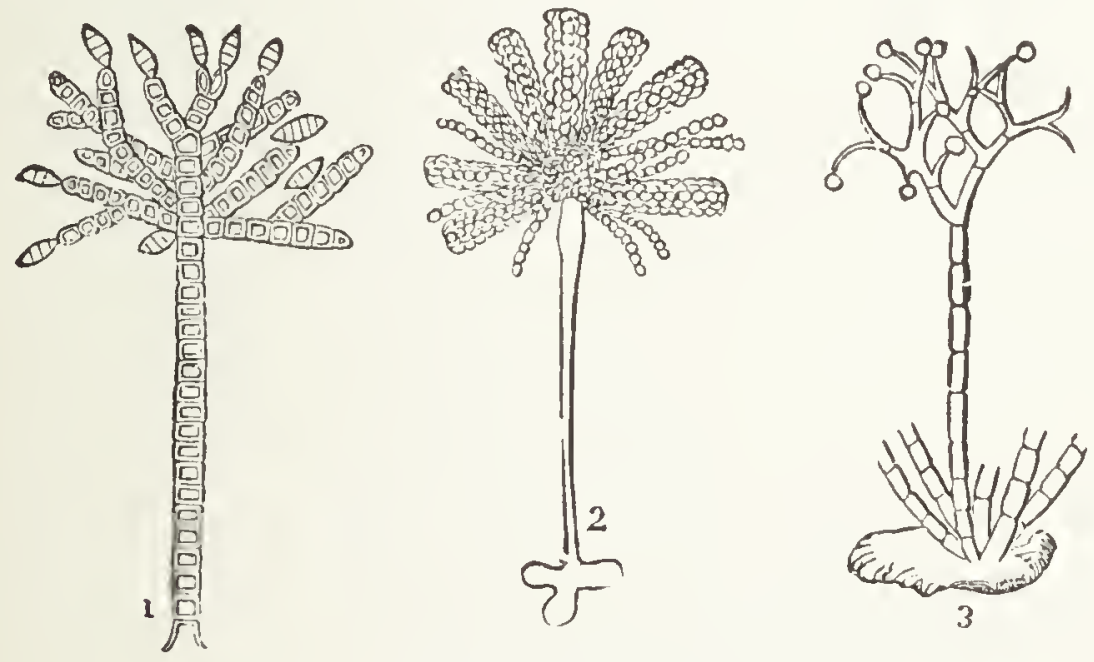

FIG. 148.-Moulds

108. Fungi, Mosses, \&c.-All the lower kinds of plants present beautiful objects for the microscope, but cannot be 
particularised. Almost any kind of ' mould' becomes, when magnified with a low power, a miniature forest of great beauty. Higher power reveals the cell arrangement. In fig. 148, No. 1 is a mould growing on the stem of a plant; No. 2 Aspergillus glaucus, the cheese-mould: No. 3 a mould found on many decayed vegetable substances.

Many of the Mosses, Lichens, and Liver-worts are also interesting objects. We may just mention the peristome of many Mosses. The product of fertilisation in these

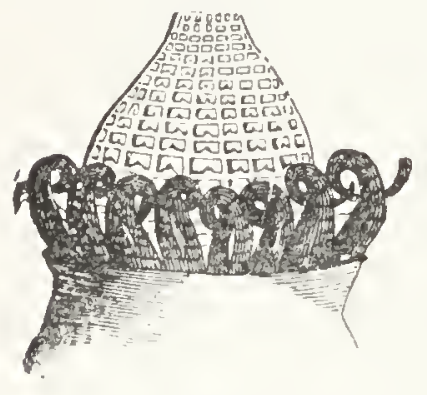

FIr. 149.

Double Peristome (fontinalis) plants is a theca or urn, which is at first closed by a lid. When the fruit is mature this lid falls off, and the mouth of the urn is then found surrounded by a toothed or fringed structure-the peristome. Fig. 149 shows a very beautiful peristome in which the fringe is double, the inner one being joined by cross-bars so as to make a kind of trellis-work. There are many beautiful varieties of double as well as single peristomes to be found in the Mosses, and a representative series would make a beautiful and interesting collection of objects.

109. Development of Sex.-We can hardly help seeing, even from the few facts mentioned in $\$ 105$, how very deep down and fundamental is the phenomenon of $\operatorname{sex}$ in the great realm of organised Nature. Even in the kingdom of plants, only in the very lowest types are two conjugating individuals alike, or can one take the place of the other; if indeed it is possible even in them, which we have seen reasons for doubting. The higher we get in organisation, the more obviously different in appearance, as well as work, do the two beings become, and the more utterly impossible for one to fulfil the functions of the other.

This development may be traced very low down. There is a filamentous Alga called Spharoplea which is composed, like Spirogyra, of many single cells joined end to end, and 
which multiplies by lengthening and division for a time. But when mature, instead of two (apparently) similar cells conjugating as in figs. 142, 143, some cells develop into sporanges, while others are filled with small active bodies with flagella, called antherozoids. A small aperture forms in the side of each kind of cell, by which the antherozoids escape from their own mother-cell, and enter the other cells, which are thus fertilised.

As we go higher the organs become more complex. There is a small fucoid plant common in marshes, called Vaucheria, which at one stage expels from the ends of its club-shaped filaments large spores, remarkable for being completely

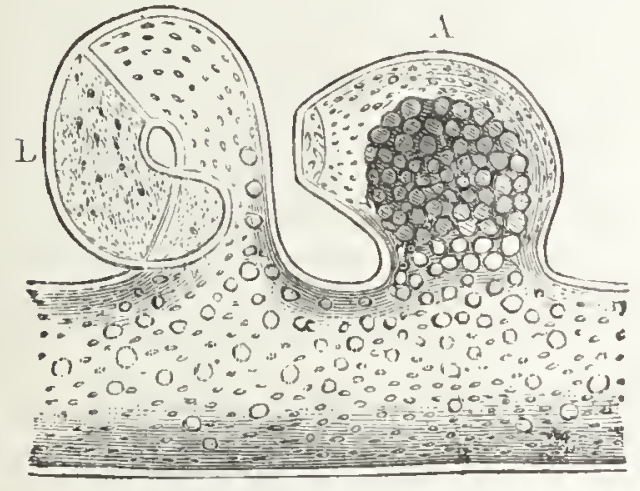

FIG. 150.- Vaucheria

Before Fertilisation

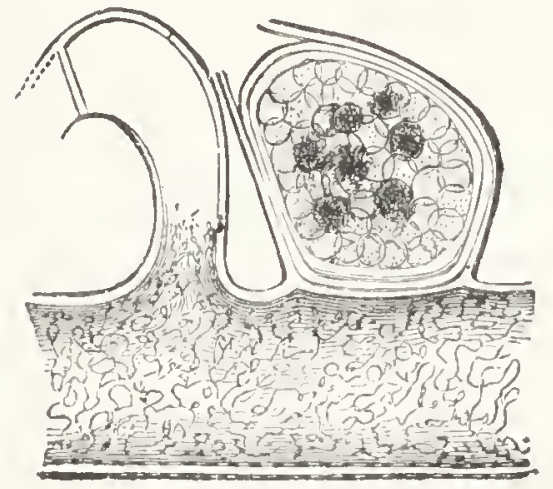

FIG. 151.-Vaucheria

After Fertilisation

ciliated all round their exterior: these are considered nonsexual zoospores. When the mature stage has arrived, the filament develops two different organs very close together, short and broad but rather curled, as in figs. 150, 151. The sporange $\mathrm{A}$ is an enclosed vessel filled with cells; the adjacent antheridium B (fig. 150) is filled with active antherozoids. The sporange bursts open first, and the antheridium soon after, when the antherozoids enter the sporange and fertilisation takes place, the result being a kind of fruit-spore as in fig. 151, containing several brown bodies. This large spore it is which sprouts into the next generation, after an inter. val, usually of three or four months.

In the Mosses and Ferns the analogous organs are further 
developed, into pairs called antheridia and archegonia, many of which form beautiful objects for the microscope, especially when cut into very thin sections.

110. Flowers and Fertilisation.-It is in the true flowerbearing plants, however, that we find reproductive organs most perfectly developed. In these the antheridia become anthers, carried on the ends of stamens, and forming pollengrains instead of spermatozoids. These pollen-grains are of very various forms, a few being shown in fig. 152. They are

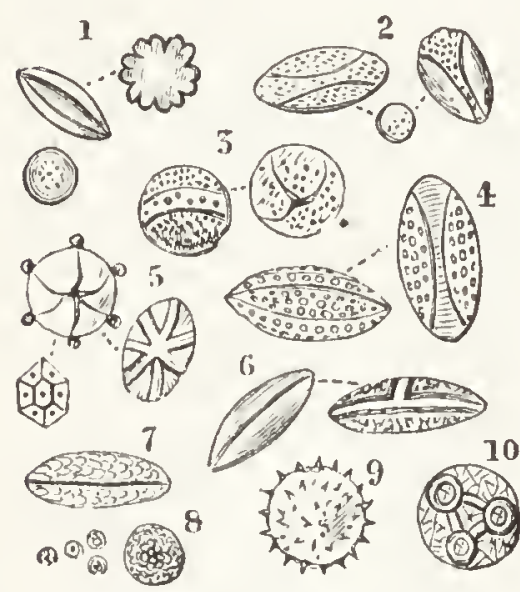

Fifr. 152.-Pollen-grains

1. Primrose 2. Auricula

3. Anemone 4. Iris

5. Clematis 0. Tulip

7. Rose 8. Buttercup

9. Hollyhock easily collected, and many make beautiful slides mounted simply in benzol-balsam. Sections across the anthers also make fine objects if skilfully made, and will show the pollen-grains within.

The female portion of the flower comprises the receptacle called the ovary, containing from one to many bodies called ovules, each of which (if fertilised) becomes a seed. From the top of the ovary extends a column termed the style, composed of soft tissue, the stigma or extremity of which exudes when mature a viscid and sticky secretion, easily seen on any fair-sized stigma with a pocket magnifier. The unfertilised stigma presents no opening whatever.

If pollen-grains are placed in water, they often burst and disperse numerous particles; and it was formerly thought that these were a kind of antherozoids, and were dispersed upon the stigma, to work their way to the ovules. Not until 1822 did Amici discover that this was not so, but that the pollen-grain, on adherence to the viscid fluid, began to push out and grow, first as a short protuberance; then a short tube; until this tube gradually grew all the way down 
between the cells of the stigma. This is represented in fig. 153, showing a section of the stigma of a Thorn-apple. The stigma with its viscid surface is shown studded over with pollen-grains, which have grown downwards as shown by the dark shading. To exhibit this in section will tax the skill of the microscopist, especially in section-cutting. The stigma of the Enothera or Evening Primrose is one of the easiest to manage; and the best way is for the operator to artificially apply pollen to mature stigmas, and then to section one morning and evening every day, till he gets what he requires. A single tube can rarely appear in the section for any great length; but the most interesting portion is that near the original pollen-grain.

This growth of the pollen-tube is a most wonderful phenomenon. Its length is often hundreds of times the diameter of the pollengrain, and may be as much as two or three inches in some plants. The question suggests itself as to how it occurs. The answer is not doubtful. The viscid sugary secretion first nourishes the pollen into growth, but the tube quickly grows beyond this source of nourish-

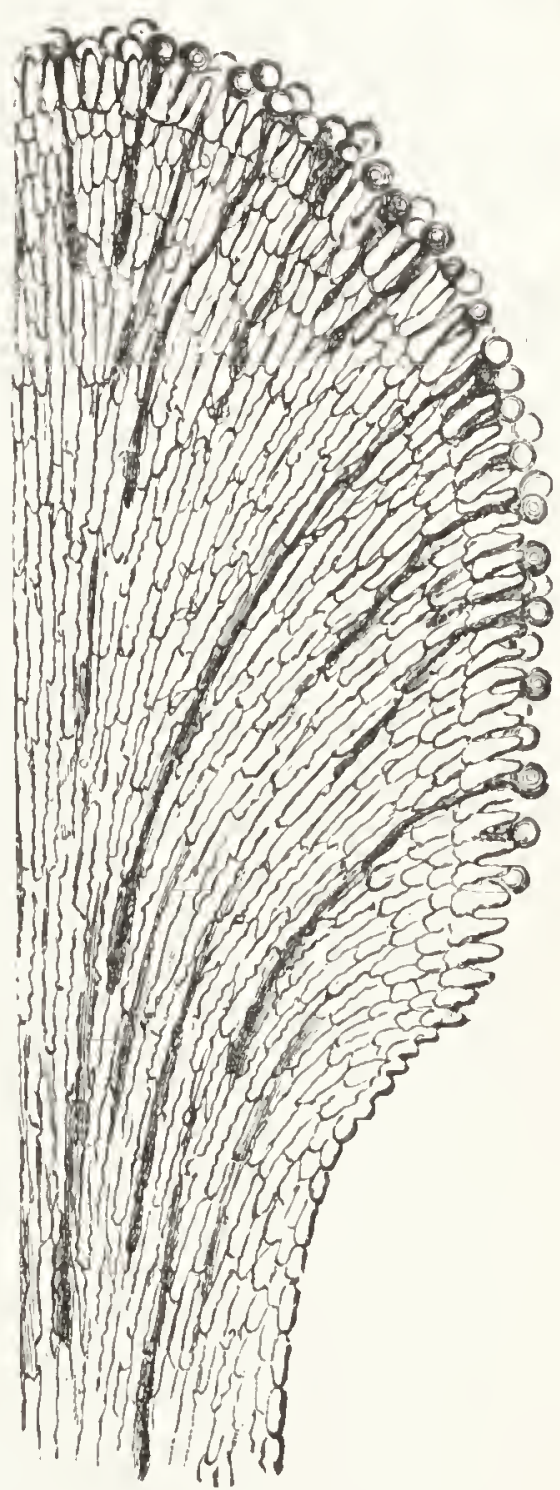

FiG. 153.-Pollen-tubes growing ment, opening for itself a kind of canal down the style. The cells lining this canal become however also charged with sugary matter, which continue to nourish the pollen-tube, and further stimulate its growth. This secretion of nutritive matter is plainly produced by the irritation caused 
by the pollen-tube, as it pushes its way farther and farther down, the phenomena being analogous to the secretion caused by Gall Insects, described in $\$ 102$. The lining of extra-nutritive conducting-tissue around or touching the pollen-tubes, can only be perceived with good lenses, in thin sections cut across the fertilised stigma.

At last the tube or tubes reach the bottom part of the style, pass into the cavity of the ovary, and there enter the

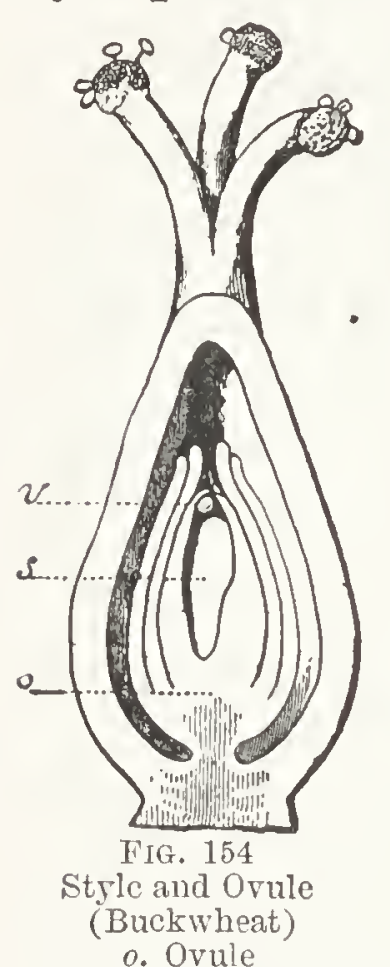

v. Germinal vesicle

s. Embryo sac minute orifice or micropyle of each individual ovule (if more than one) as seen in fig. 154, which represents the style and single ovule of buckwheat. Thus the germinal vesicle in the embryo-sac is fertilised, and certain further changes commence, which change the ovule into a seed or fruit, embodying for the next generation all the work of the past, all the hope of the future.

It cannot be here detailed how everything in Nature is made to bear upon this end, in the greatest variety of ways. It is easy to write volumes upon the 'Loves of the Plants,' and such have been written. The scents and colours of flowers are all to serve this end; the visits of insects, with nectar to tempt them, and with colour and odour to attract them, being relied upon to bring the pollen of one flower to the stigma of another. In not a few cases one insect alone is able to do this. In other cases the wind performs the hymeneal office, and appendages to catch the wind are provided. In many cases the pollen fertilises the stigma of its own flower. Anthers and stigma combined in one flower are very common, but it is also common to find the sexes on altogether separate flowers, as in the willow and yew. But all such marvellous instances of adaptation belong to Botany rather than Microscopy. 
Sections of flowers, and ovaries, and buds, both transverse and longitudinal, are very beautiful objects. Being soft, they are prepared with no special difficulty.

111. Stems.-A few words must suffice for the structures found in plants. Of the preparation of sections of Stems, in general, and their staining or double staining, sufficient for the purposes of this book has been said in \$§77-79. Shoots or branches from $\frac{1}{4}$ to $\frac{1}{2}$ inch diameter are most suitable; but this must depend upon the rate of growth. If possible, three sections should be prepared of each-one transverse, one longitudinal in a radial direction from the centre of the shoot, and a third also longitudinal, but cut at a tangent to that centre. In regard to exogens, or those plants which add rings every year, it is a good plan to prepare three transverse sections, of one, two, and three years' growth. The common pine shows the annual rings clearly.

Endogens, on the contrary, exhibit independent bundles of fibro-vascular tissue over the area of the stem. Many of them, well cut and stained, are of exquisite beauty. Roots also offer many interesting sections.

112. Vessels. - The stems and other main parts of plants mostly consist of longitudinal vessels, which are formed essentially by the elongation of cells and thickening of the cell walls. We cannot go into detail; but fig. 155 shows one very characteristic type, in which the harder thickening material is arranged in spiral form, just as the chitine in the tracheal tube of an insect (fig. 116) shown for comparison in the same figure. Spiral vessels are easily, after a little maceration, teased out of a piece of the stem of the common rhubarb, and of many other plants, especially from the larger veins of soft leaves.

If the rings are separate, and more or less complete, we have anmular vessels, or the thickened walls may be dotted over with thinner spots, which give punctated vessels. In some plants, Ferns especially, the walls of many vessels are 
more or less polygonal, and the rings very close, and connected by very short longitudinal fibres: these forms are known as scalariform vessels.

In woody fibre the cellwalls are much thickened and hardened. The most interesting form of woody tissue is found in deal and other coniferous woods. Here each long cell is studded with thin spots surrounded by concave depres-

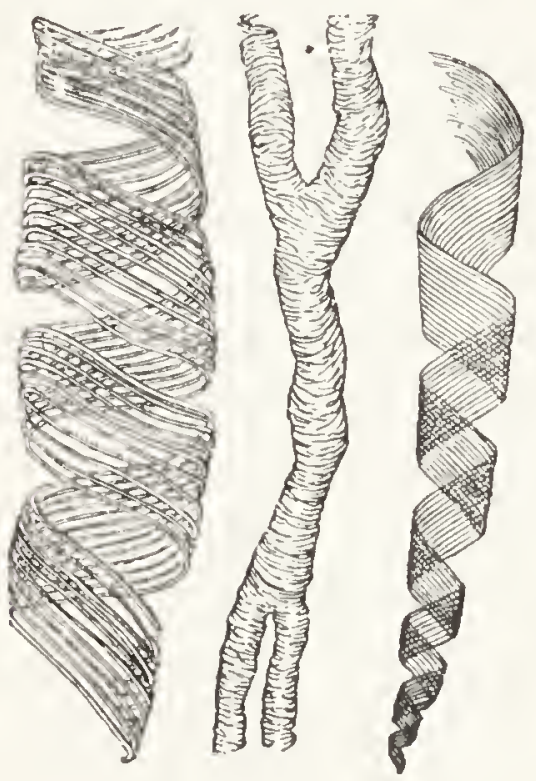

FIa. 155.-Spiral vessels (Trachea of an insect shown in centre for comparison)
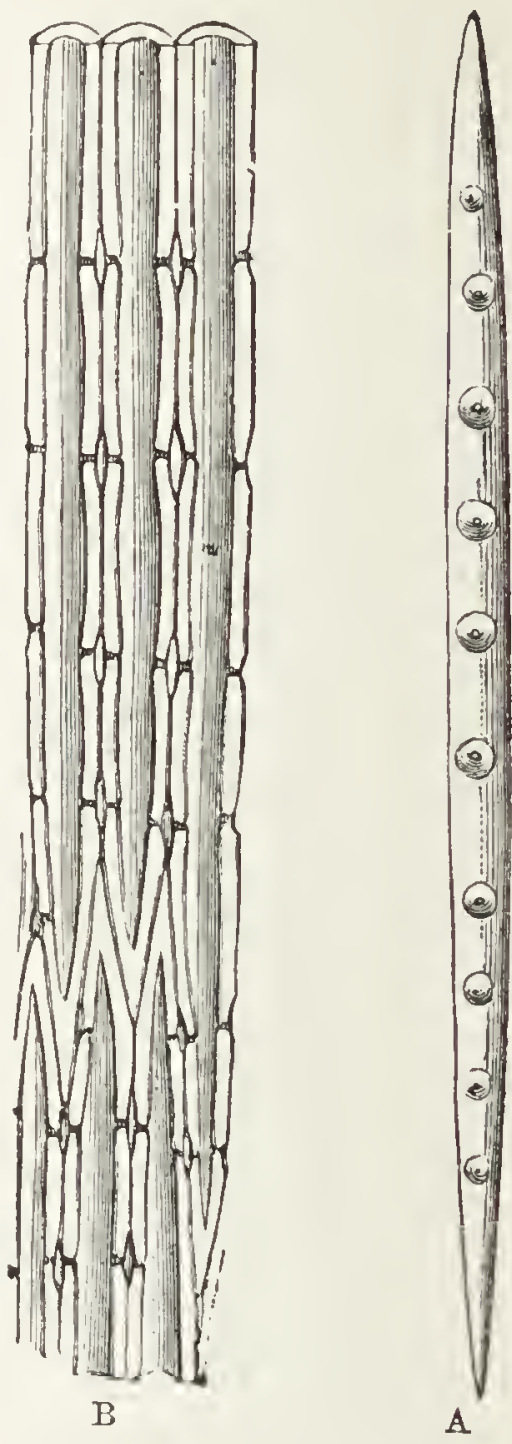

FIG. 156.--Pitted vessels

sions, on the flat faces which are turned towards the medullary rays, or radial planes from the centre of the stem. The flat face of the cell with the pits is shown at $A$, fig. 156, and the lenticular cavity formed by two pits (which always face each other) is shown in section at B. A very thin slice from a lucifer match will show these pits; but to get the two positions perfectly, sections must be cut both radially and tangentially as regards the stem. 
113. Leaves.-These offer many interesting objects. Separating and staining a piece of the under cuticle, or surface layer of cells, this will in most cases show under a quite low power the Stomata or apertures, leading into cavities in the cellular tissue, by which plants 'breathe' (fig. 157, A). With a higher power it will be seen (c, which shows the little piece marked $B$ in $A$, further magnified) how each aperture is bounded by two kidney-shaped 'guardcells.' The cuticle will also exhibit cellular structure plainly.

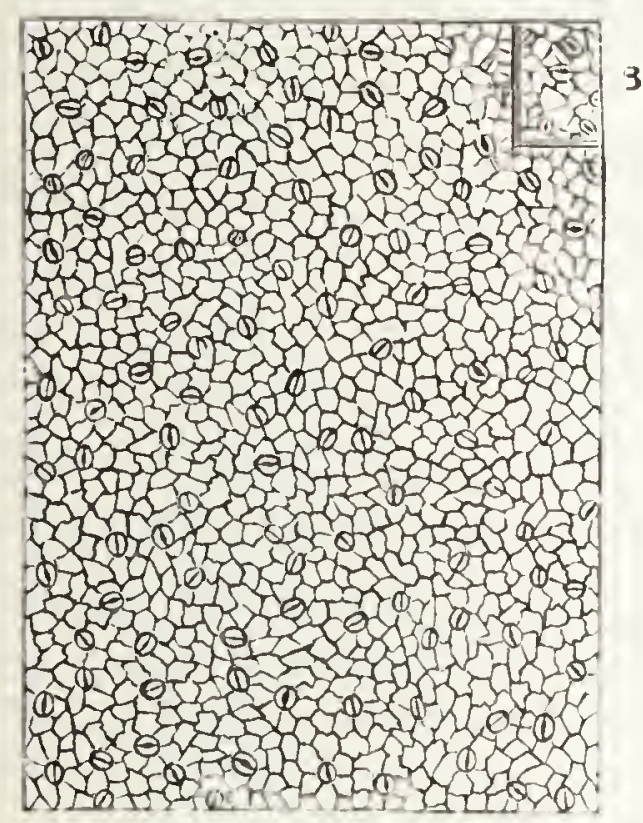

A. $\times 100$

FIG. 157.-Stomata of Lilac Leaf

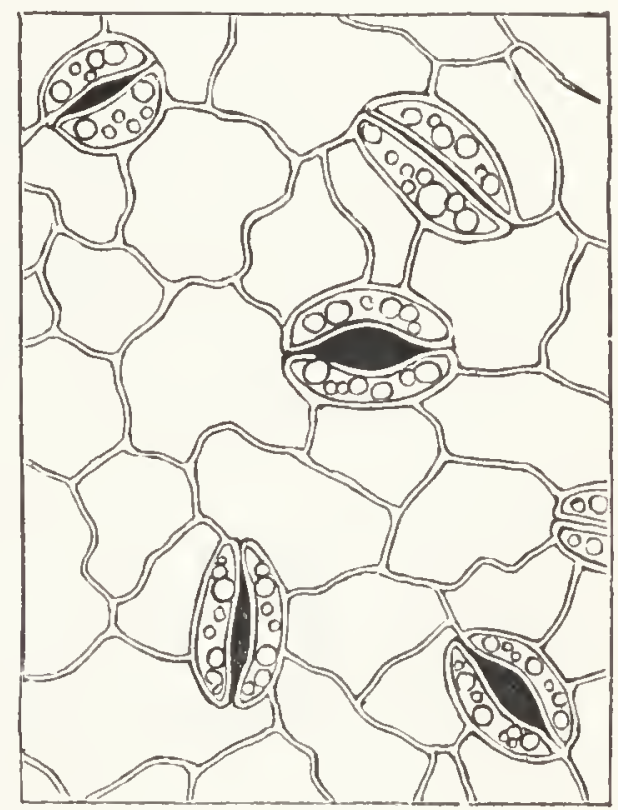

C. $\times 700$

Vertical sections of leaves should also be prepared, and are very interesting. Under the cuticular layer, is usually one of deeper and narrower cells, often termed 'palisade' cells (fig. 158). Beneath this the cellular arrangement varies much, but as a rule the upper layers are dense, whilst amongst the lower layers are many cavities, into which the stomata open. A great deal depends on chance; but out of a few sections one or two should exhibit some of the stomata in section, as in the figure. Some of the thicker leathery leaves, such as laurel, or of the India-rubber plant (Ficus elastica) make fine vertical sections with the least trouble. 
Portions of the fronds of Ferns may be mounted either dry, or transparent. Many petals of flowers, which are forms of leaves, are lovely objects. A favourite one is a petal of geranium, stripped of its cuticle, then dried, immersed in turpentine for two hours, and mounted in balsam. The petal from a flower of the common Chickweed is another beautiful object, and shows in the 'veins' spiral vessels in their natural position.

The cuticles of many leaves are studded with hairs of various shapes. Many cells of leaves and other parts contain crystals, either singly or in masses. In others there

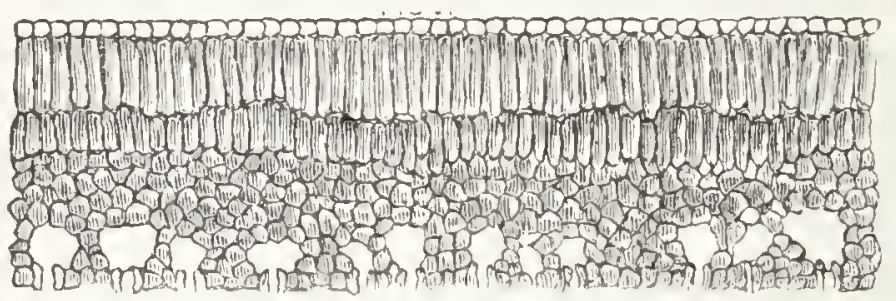

FIG. 158.-Vertical Section of Lilac Leaf $\times 100$ are grains of starch, which phenomenon is best shown in a thin section of potato. Hard tissues such as the 'stones ' of fruits; thin cuticles such as enclose the edible part of a walnut ; pith ; rind; all these and other structures present various forms of tissue built up of cells, for further details of which reference must be made to works on Structural Botany or the Physiology of Plants.

114. Cyclosis.-We must conclude this chapter by reference to one phenomenon of living cells, which more than any others impresses the observer with the idea of vital activity. This is the circulation in each cell of the living protoplasm, in a current round the outer walls, which can be best traced by the current carrying with it green particles of chlorophyll. The probability is that all cells containing fluid protoplasm present more or less of this motion (which must not be confounded with the circulation of sap through the whole plant: it is simply a current round the interior of each cell). But only thin cells with transparent walls are suitable for observing the phenomena.

The hair of a Nettle, or still better of the Tradescantia 
Virginia, detached and put on a slip with a drop of warm water, shows this phenomenon well. So do the terminal shoots of the aquatic plant Chara, or the thinner ones of the allied Nitella. A leaf of Anacharis treated in the same way shows this protoplasmic movement (termed 'cyclosis') well, and with no trouble, in the cells up the midrib, or round the edge of the leaf. But it is best shown, though with rather more trouble, in a piece of leaf of Vallisneria. A thin slice should be shaved (with a lancet is easiest) from the surface of the leaf, so as to come as near as possible to a single layer of the cells, and put on a slip with warm water. Such a thin section is nearly transparent, and the cells are on a larger scale and more regular in form. A fair quarter-inch with a moderate eyepiece, or a high-class half-inch or

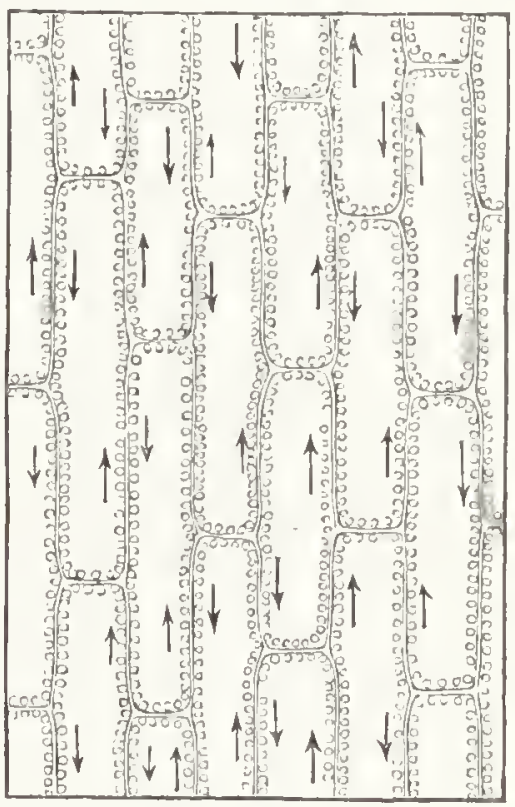

FIG. 159.

Cyclosis in Vallisneria four-tenths with a high-power ocular, will exhibit the motion of the chlorophyll granules well, as in fig. 159, which is drawn under a half-inch apochromatic.

Generally the detachment of the piece of plant, or of the hair or leaf, as may be used, appears at first to paralyse the motion, as if the cells were really killed. This is however only temporary, and if the leaves or small slices are placed for an hour or two in a small tube full of warm water, or carried in a pocket next the body so as to be gently warmed, the cells are revived, and the motion recommences. 


\section{CHAPTER XI}

\section{VERTEBRATE PHYSIOLOGY}

That study of the most minute structure of the human or other body which is termed Histology, does not belong to the purposes of this work. Medical or biological students will work out such studies by the aid of the treatises of Schäfer or Klein, and.such material as is provided in class. So far as the human body is concerned, material for personal section-work or dissection will rarely come in the ordinary reader's way; and it must suffice to indicate in this chapter a few typical organs or structures of which the microscopist will probably desire to possess a few purchased slides, as being of popular interest. ${ }^{1}$

115. Blood.-The examination of a drop of blood is an exception, being so easily made. Winding a few threads round a finger below the nail, a prick near the side of the nail with a fine needle is hardly felt, and is followed by the desired drop, which can be transferred to a slip. If the cover be placed on the drop so as to form a thick layer, the red corpuscles show a great tendency to gather into rolls adhering by their flat sides like a pile of shillings (fig. 160, A). But if the cover-glass is laid on the dry slip and pushed along till the blood is drawn under by capillarity, a thin layer is secured, when the individual corpuscles stand out

1 For those interested in Histology as such, an exception may be made to the recommendation on p. 136 not to purchase 'sets' of slides. From any of the metropolitan microscope houses a set of six dozen slides, mounted in balsam, in a pine cabinet like fig. 70, and representing (according to a reference-list) nearly all the important organs and tissues of the human body, fan be obtained for two guineas. They are known in the trade as 'Jones' sets,' and are largely used by medical students and teachers. The sections are admirably prepared. 
separately, c. The little disks, averaging $\frac{1}{3500}$ inch in diameter, have rounded edges, and are rather hollowed on the sides. This concave lenticular form results in a difference of focus showing either light or dark centres.

The corpuscles of the blood of fishes, reptiles, and birds are larger, oval in form, and with a very distinct nucleus. Those of some reptiles are very large in proportion, and slides are procurable of any dealer showing the nuclei stained green, with the rest of the corpuscle stained red.

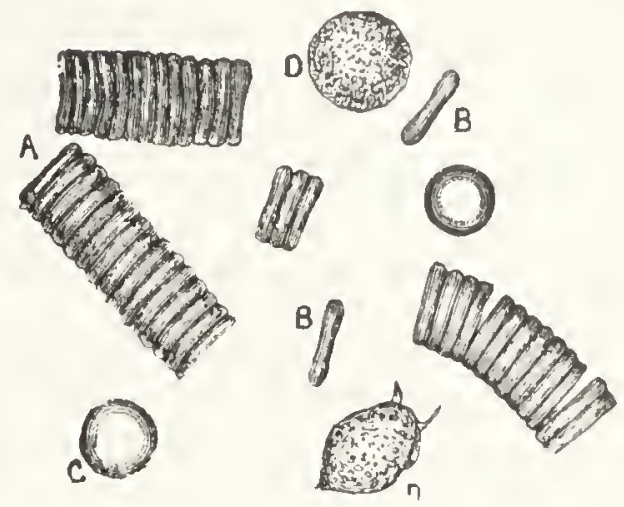

Fig. 160.-Blood Corpuscles A. Roll of red corpuscles BB. Red corpuscles in profile C. Red corpuscle tlat DD. White corpuscles

Here and there appear among the round disks, rather larger corpuscles D D, not so smooth at the edge, and at times with distinct protuberances. They rarely average more than 1 in 250 to 400 of the red ones. If the slip can be kept warmed to blood heat, they are found to change their form and place as in fig. 161. In short, except that they are much smaller than the ordinary amoba of fig. 86, they
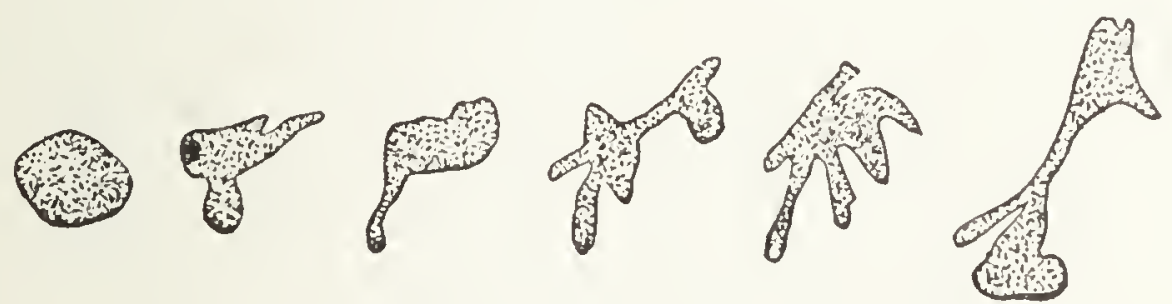

FIG. 161.-Amœboid Changes in White Corpuscles

appear to behave exactly the same in every respect. They are, in fact, distinct living bodies of the same kind, taking food also in exactly the same way. Especially have they been observed to feed upon such bacteria as infest the body; and when irritation or inflammation is set up anywhere, by the attack of these disease-producing germs, the white 
corpuscles or leucocytes rush to the spot from distant points, and appear to attempt to devour them. The point is still debated; but a large school of biologists and medical men believe that resistance to such attacks of disease-germs, or recovery from the attacks, depends upon whether the white corpuscles of the blood can succeed in devouring the germs before fatal mischief is done.

116. Circulation of the Blood.-The display of this is always interesting. It is usually shown in the transparent web of the foot of a frog; but the tail of a tadpole, or tail or

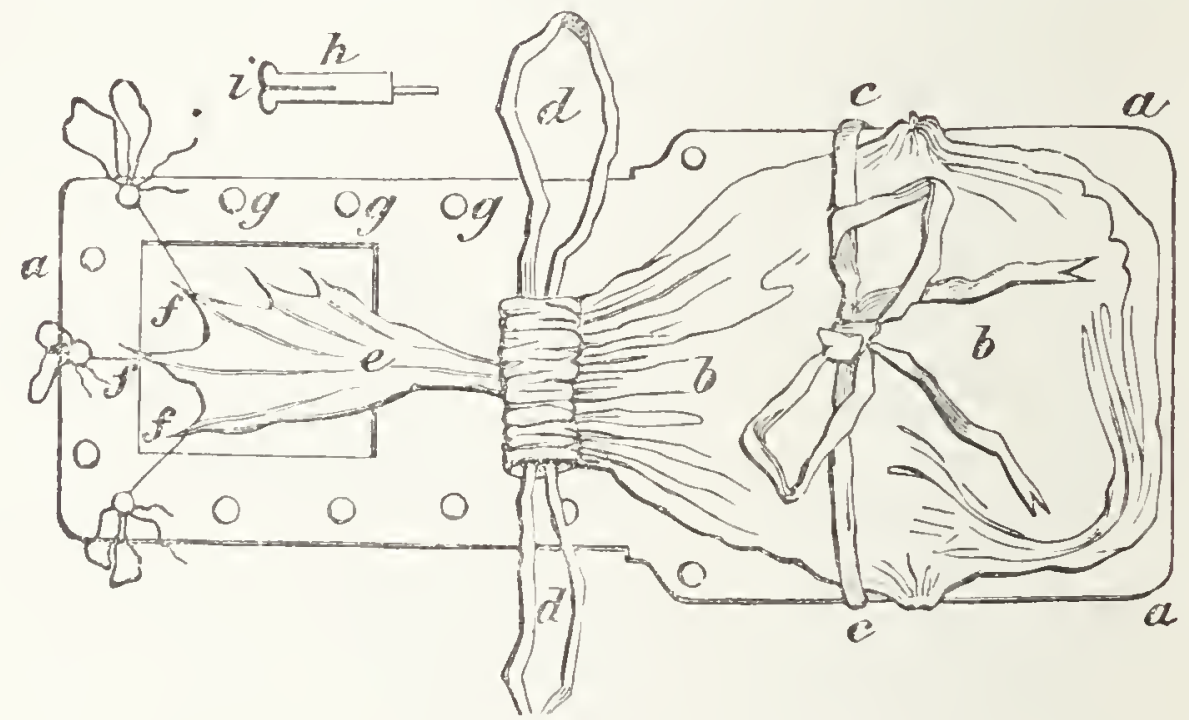

FIG. 162.-Frog on Plate

fin of a minnow, are also used as convenient. We will take the frog. The orthodox manner of treating him was to have a 'frog-plate' of brass a a a (fig. 162) with holes $g \mathrm{~g} g$ round one end, and an aperture across which the foot is to be stretched. The frog is put head first into a bag $b \quad b$, whose neck can be drawn round the one leg allowed to protrude by the strings $d d$, and by tapes $c c$ sewn to the bag, it can be tied securely round the plate, or what is better, to studs projecting from it. A few pins $h$ with slots $i$ cut in them are provided to fit the holes $g g$. To the principal toes $f f f$ threads are tied: then these threads are stretched out, stretching the web with them, and the threads being turned 
two or three times round the pegs and then pulled down through the slit $i$ in each, the whole is fastened. The bag should be of linen or cotton, not waterproof, and be wetted, or a little wet moss put in with the animal; and the web of the foot also needs wetting occasionally. If the bag or the tapes be tied too tightly, the circulation will sometimes stop, and it should be loosened a little. Mere fright sometimes seems to paralyse the animal; but there is very seldom any difficulty.

But most demonstrators prefer Dr. Carpenter's plan of using a plate of cork, with a hole half an inch diameter in the centre. The threads attached to the toes have little loops made in their ends, or are tied round common pins; and these pins are stuck into the cork so as to stretch the web, as with the plate. An inch power is amply sufficient.

117. Capillary System.-The circulation thus seen is near the smaller extremities of the veins and arteries, where the two pass into each other through the capillary tubes. With a little adjustment the capillary connections themselves can be brought into view, the diameter of these tiny tubes being in many cases only sufficient for the free passage of

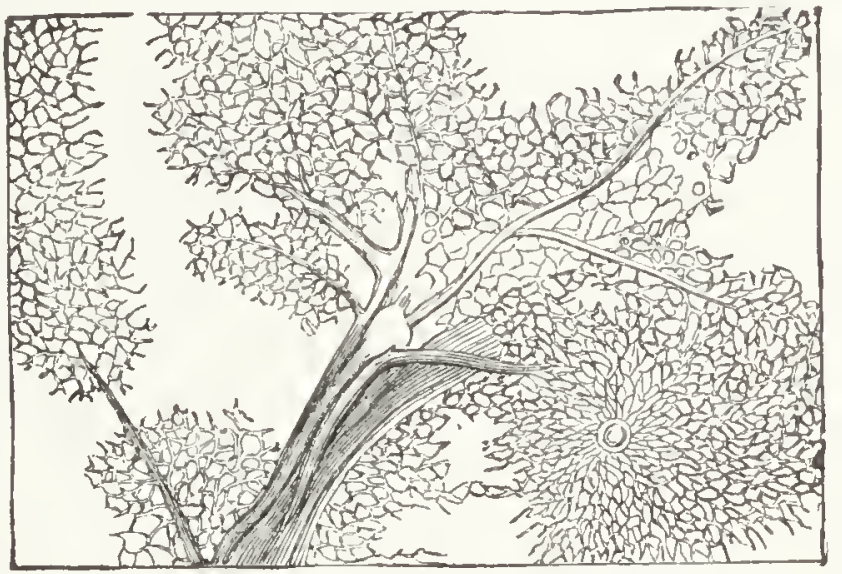

FIG. 163.-Capillary Vessels in Lung one corpuscle at a time. To exhibit these capillary bloodvessels, sections are prepared of tissues in which the bloodvessels have been injected with carmine, so as to show up in bright crimson. Fig. 163 represents a small portion magnified of the capillary system in the lung.

118. Ciliated Cells.-The amœboid white blood-corpuscles are not the only case in which we find grouped, in the higher 
animals, individual cells reproducing the lower forms of life. It is true that the cellular construction of a vertebrate's body is not (to a very low power) so conspicuous as in the case of plants, in which it cannot be overlooked; but the use of moderately high powers speedily reveals that here also we have a complex life, embracing in it the individual life of innumerable single cells. We cannot enumerate all the various kinds; but one of the most interesting (from this point of view) is the ciliated cell.

The ciliated animalculæ were briefly described in $\$ 85$, and the part the minute cilia played in the motion of the

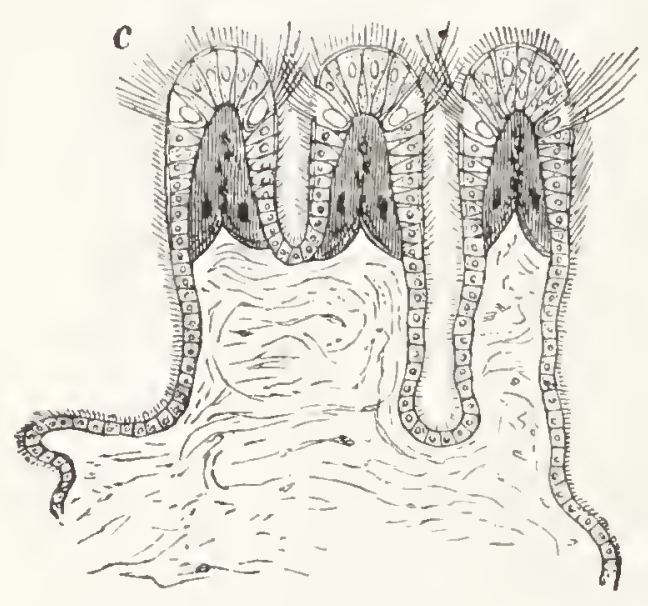

FIG. 164.-Gills of Mussel

c. Cilia animal, or (by causing currents in the surrounding fluid) procuring food or assisting respiration, was alluded to. Taking now an example far above an Infusorian, yet far below any vertebrate animal in structure, if we examine a section through the gills of a mussel, with a high power, we shall see (fig. 164) that the exterior of the gill-leaves or plates is composed of longish cells in close contact, each with a nucleus, and with their outer ends furnished with cilia $c$. These are in incessant motion, and by this motion the fresh water is swept into the gills, and the breathed water expelled. This office is what we should probably expect. The motions of the cilia average some ten or twelve per second; and that the individual cell-life is apart from the higher or organic life, is proved by the fact that the cilia are seen to move, hour's after a piece of gill has been detached from the animal. ${ }^{1}$

1 Dr. Carpenter states that the eilia have been seen in motion on the trachea of a criminal seven days after decapitation. And detached ciliated cells will swim freely about in warm water like an Infusorian. 
But it is wonderfully interesting to know that we find similar ciliated cells in the highest Vertebrates, including ourselves, and that they perform most important functions. In this case they line interior parts of the body, which arises from the mode of embryonic development. If the germ of a hatching egg be kept under examination, the first trace of the future 'plan' of the animal is an open groove down the centre. This groove gradually grows deeper, and at last closes in, into a tube. Thus the originally outside layer of cells, becomes an inside lining. The internal organs are developed from the cavity thus formed. It is only on some of these surfaces, which are kept moist by thus becoming internal surfaces, that the cilia survive. Fig. 165 illustrates a few forms, a being from the lining membrane of the nose, B from the trachea of acat, and $\mathrm{C}$ from the mouth of a frog. They belong to the delicate lining known as epithelium. Every cell is seen to be distinct, and to have its own nucleus; but the cells differ in shape. When oblong and regular as in A, they are known as
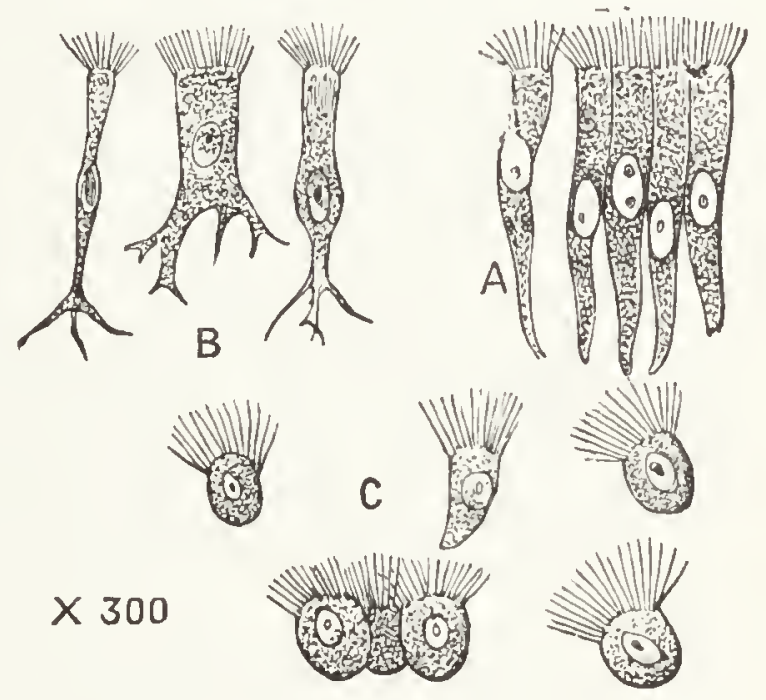

FIG. 165.-Ciliated Vertebrate Cells 'columnar' epithelium, and when in addition ciliated, as here, ' ciliated ' epithelium. Only a most skilled section-cutter can furnish sections showing these cells; but if the reader has a good $\frac{1}{4}$ or $\frac{1}{6}$, he can probably procure detached ciliated cells by scraping the inside of a frog's cheek with a spoon, ${ }^{1}$ and placing a drop of the mucus upon a slip with a cover.

The great interest of these cells lies in their position and

1 No more pain is caused than in scraping one's own. This will also produce epithelial cells; but in man these are not ciliated. 
the work they do. The main positions they occupy are along the spinal cord or marrow, a narrow tube in which is lined with cilia; the cavities of the brain; the windpipe, trachea, and bronchi, and nasal tubes-i.e. the respiratory passages ; and the ear passages. How these originally outside appendages came to be on inside places, we have seen.

Their office is a cleansing and freshening office. In the case of the respiratory passages, it has been ascertained that the cilia keep the fluids lining those passages in continuous motion towards the mouth, thus getting rid of dust, or effete and diseased matter. It is when the offending matter gets too much for this action, or when the action of the cilia has not sufficient vigour, that we get distressed, and have to cough the matter up. The lining of the nervous cavities probably has to be cleansed in the same way.

119. Muscle.-Of the more obvious structures of the vertebrate body, the first to occur to mind is muscle-the

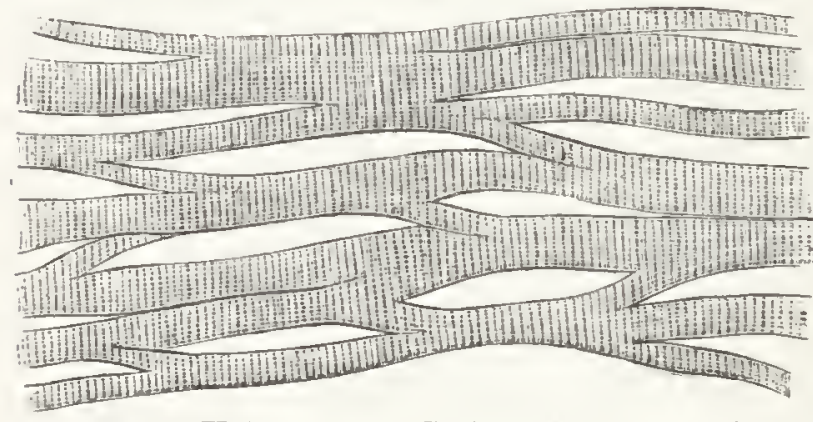

FIG. 166.-Toluntary or Striped Muscular Fibre substance of 'lean'

flesh. This is of two great classes, sharply distinguished. In all cases where the motion produced by the muscle is voluntary, the fibres are striped or striated as in fig. 166. The coarseness, and some characteristics of the striation, may differ in different classes of animals, as in mammals, reptiles, fish, and insects ; but striation is common to all. On the other hand, where the motion is not voluntary, as in the intestines, the fibres are plain or unstriped.

Muscle can be prepared easily. A bit of either raw or boiled flesh may be teased out with two fine needles into the finest possible fibrils, under an inch power of the single or dissecting microscope. Raw flesh is sometimes teased out more easily if first macerated in a weak solution of chromic 
acid. The flesh of the pig or the frog yields good fibres, as do lobsters and crabs. Some insects furnish muscle with coar'ser striation, the Dyticus or great Water-beetle being a favourite. The striations are thought to show more plainly, if the muscle is mounted in glycerine or glycerine jelly: but on the whole balsam is to be preferred.

120. Bone.--Sections of bone are interesting. Fig. 167 is a transverse section of human bone, showing first a series of very small tubes (averaging $\frac{1}{300}$ inch diameter) known as

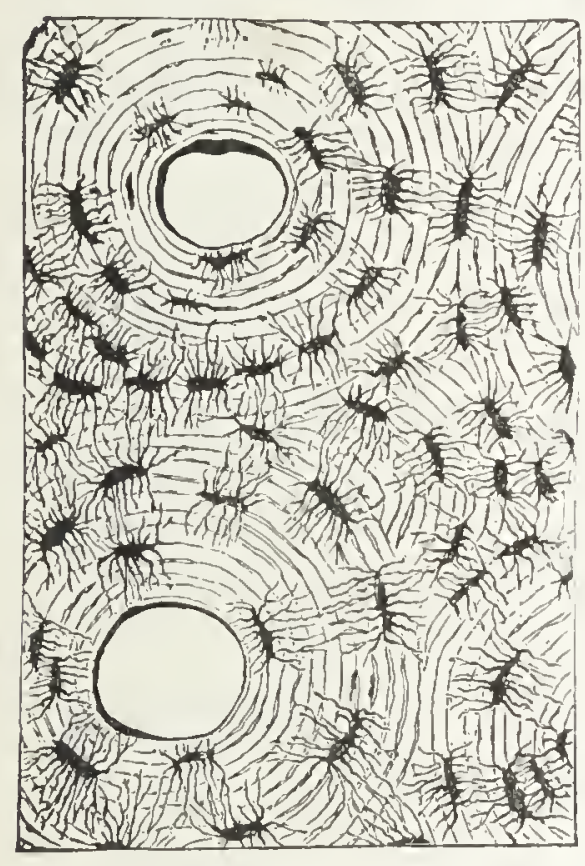

Fig. 167.--Human Collar Bone $\times 100$ 'Transverse Section

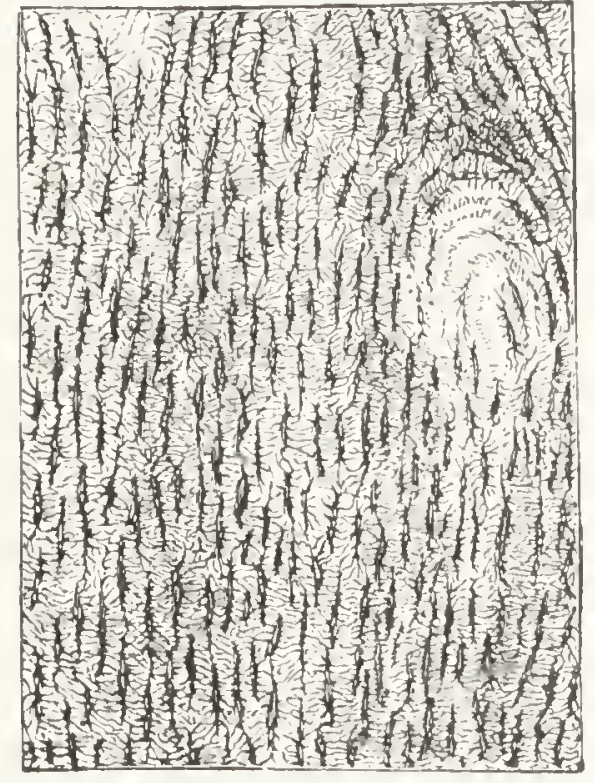

Fig. 168.-Fore-arm of a Turtle $\times 75$ Transverse Section

the Haversian canals, which are in connection with the great central cavity (when there is one, and like it are filled with mar'ow), and supplied with blood-vessels. Round these are concentric lamella of the organic structure, infiltrated with earthy matter. Between these lamella are spaces called lacunæ, shown by the black spider-like spots, from which radiate in all directions a multitude of very fine canals-caniculi. The lacunæ are generally about one-third deeper than they appear broad, and the proportion between their apparent length and breadth (the section being sup- 
posed to be cut across the Haversian canal) differs in the great classes of animals, so that a bit of bone will usually tell of itself whether it belonged to mammal, bird, or reptile. Thus, in the reptile bone shown in fig. 168, the lacunæ are longer but narrower than in the human bone: also the bone being a flatter one, they tend more to straight parallel rows. By these lacunæ and their caniculi, which are filled with protoplasmic matter, communication is kept up throughout the bone with the Haversian canals and larger hollows, and vital action maintained. It is remarkable that there should be a general correspondence in the size of the lacunw, and those of the blood-corpuscles of the animal to whom the bone belongs: but the caniculi are far too small for blood-circulation.

121. Skin and Hair.--Passing now to the external surface of the body, the skin presents many points of interest. Transverse sections or layers will display the ridges and furrows as in fig. 169, and at intervals the mouths of the

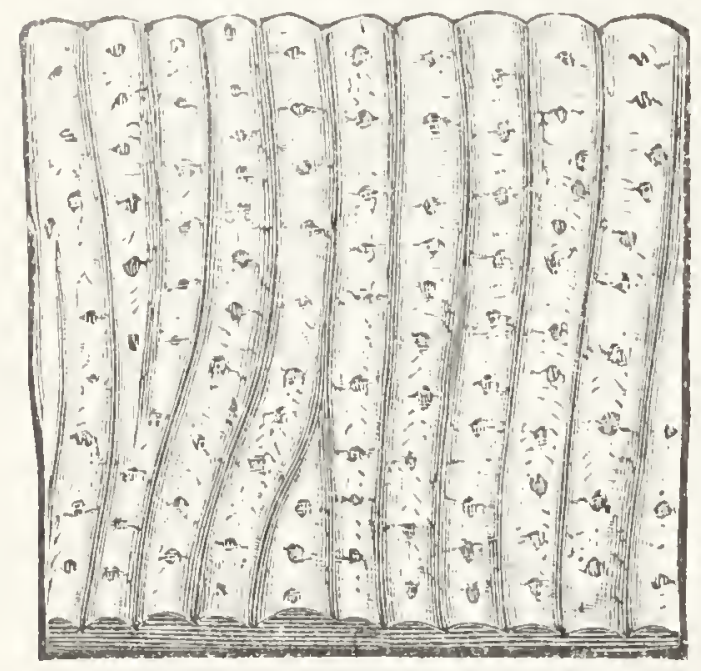

FIG. 169.-Surface of Sliin $\times 75$ pores by which perspiration is exuded. These pores were counted by Dr. Erasmus Wilson, and found (on the palm of the hand) to number rather over 3,500 in a square inch. On an average the tiny tubes of which they are the mouths, penetrate to a depth of about a quarter of an inch, making a total length of drainage-tubes in that small area, of about 73 feet! It is easy to see the serious results which must follow if such an amount of drainage is not kept free and open; and what work must be thrown upon other excretory organs (such as masal passages or kidneys) beyond their proper share. 
Fig. 170 is a vertical section of skin. At $a$ is the epidermis, or scurf skin, raised in ridges, the outer part of which consists of dead horny matter which is constantly peeling off, as can be seen very prominently in a section of the skin on the sole of the foot. The inner portion or Malphigian layer receives projections or papilla $d$ of the true skin beneath, which are supplied with capillary blood-

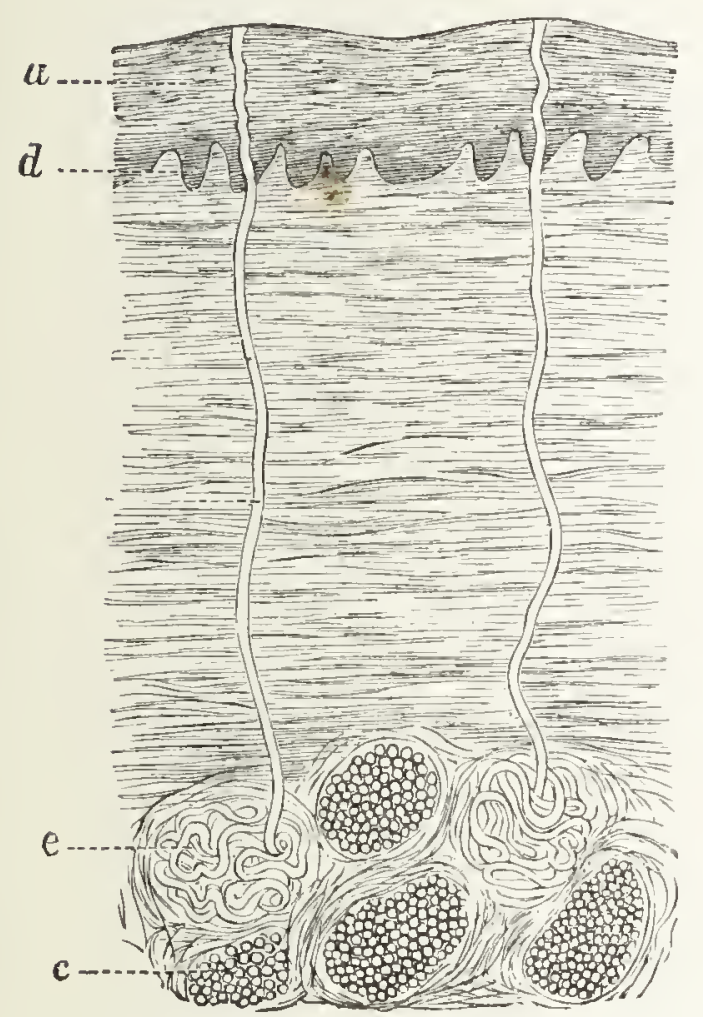

Fig. 170.-Vertical Section of Skin vessels and nerve endings (see fig. 173). Deeper down in the true skin are the perspiratory glands, $e$, each gland a coiled up narrow tube, which finally passes out to the sur-

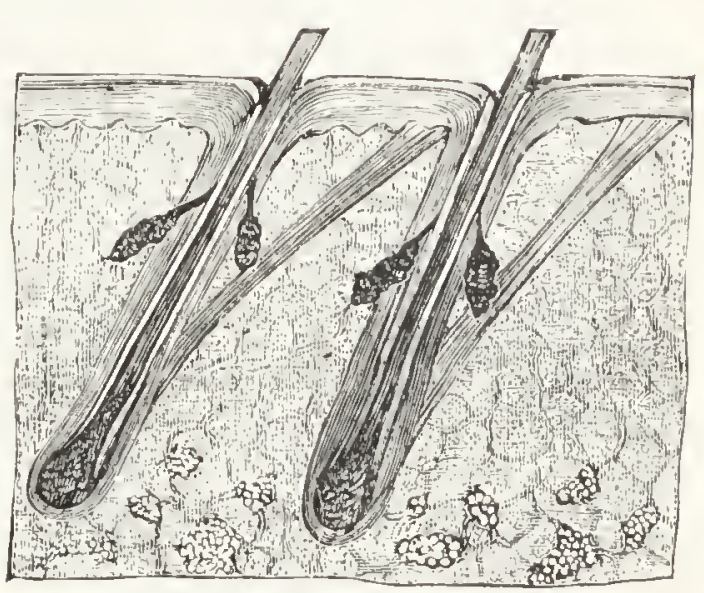

FIG. 171.-Scalp with Hairs

face, assuming through the epidermis a cork-screw spiral form. The glands lie amongst masses of fat-cells $c$.

The skin is also furnished with sebaceous or oil-secreting glands, not very different in structure from the preceding. They are most conspicuous round the roots of hairs.

Fig. 171 is a section of the skin of the scalp, showing two hairs and hair-follicles. The sebaceous glands and muscles by which each hair can be moved, are clearly seen. A double-stained section of the scalp is a very beautiful object, as are double-stained preparations cut so as to give transverse sections through the hairs and hair-follicles. 
Many hairs themselves are very interesting, and the most marked varieties are always to be purchased. The

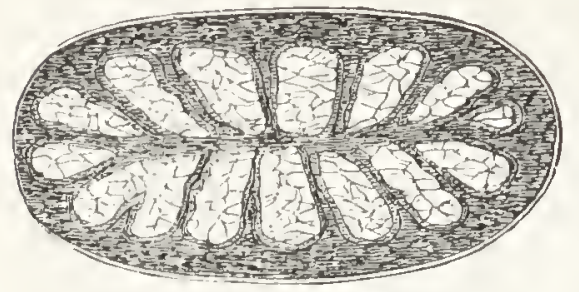

FIfr. 172,-Hair of Peccary Transverse Section whiskers of lions and tigers give very large transverse sections. Fig. 172 shows the very peculiar arrangement of the medullary and cortical portions of the hair of the peccary. The hair of the sable is remarkable for the very large rounded cells occupying the centre. A most beautiful object is a good double-stained and 'injected' section of the lip of a cat or rabbit, showing the sensory hairs, and their follicles and appendages.

122. Nerve Endings. - This leads naturally to the organs of scnsation, through which we are impressed by the external world. We will only mention here the sensations of touch, of taste, and of sight.

The skin itself is an organ of feeling, and fig. 173 is a much more magnified view of the papilla seen in fig. 170 ,

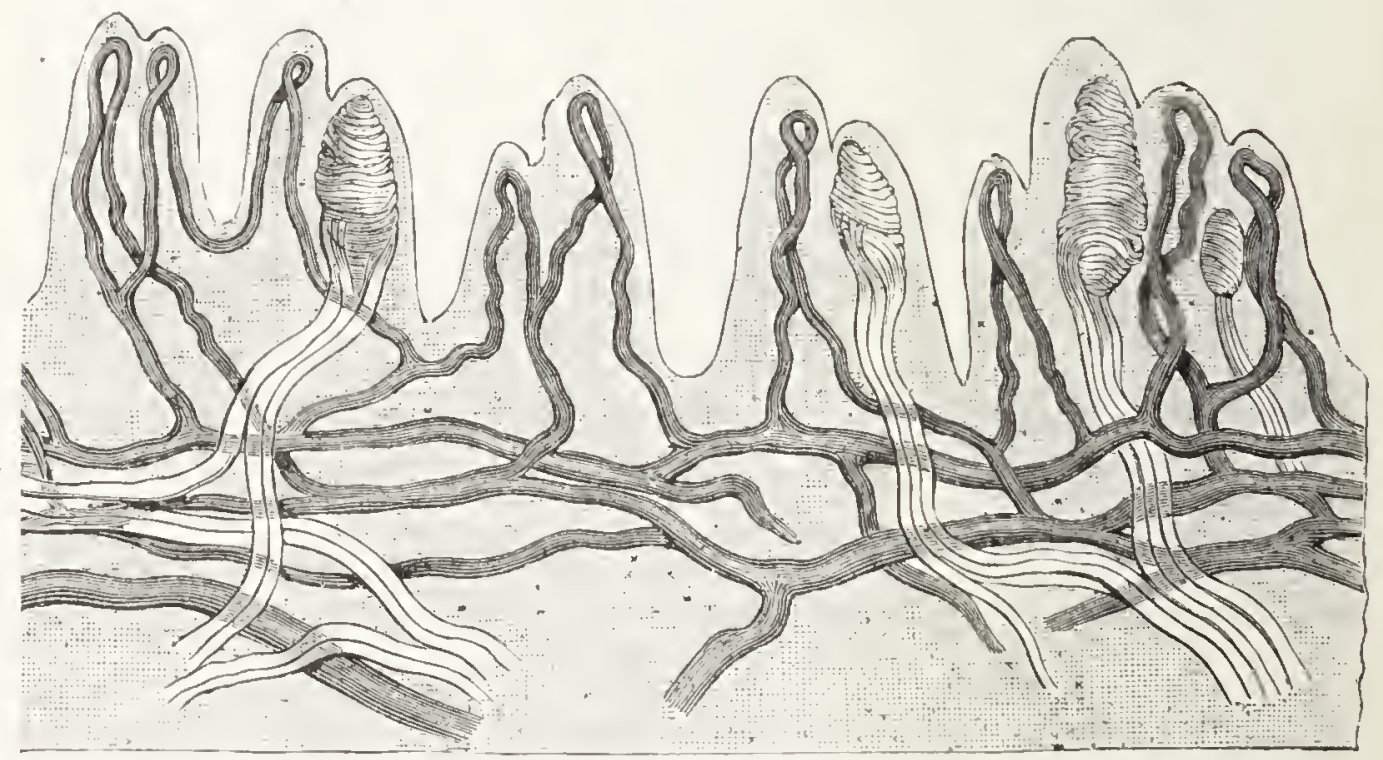

Fra. 173.-Tapillæ of the Skin, showing capillary vesscls and touch-bodies

with the epidermis stripped away. Every one contains a loop of capillary blood-vessels, shown by the darkest shading. Many of them contain also the ends of minute nerves, shown 
in white, and which are twisted into oval bulbs. These are generally considered and termed 'touch-bodies.' It is by no means easy to find a section of skin which exhibits one or more of these touch-bodies really well, as they elude the section-knife owing to their softness and minute size.

The more delicate sensations, as we should expect, require more complicated mechanism. The terminal taste organs are chiefly found in a certain region of the tongue covered

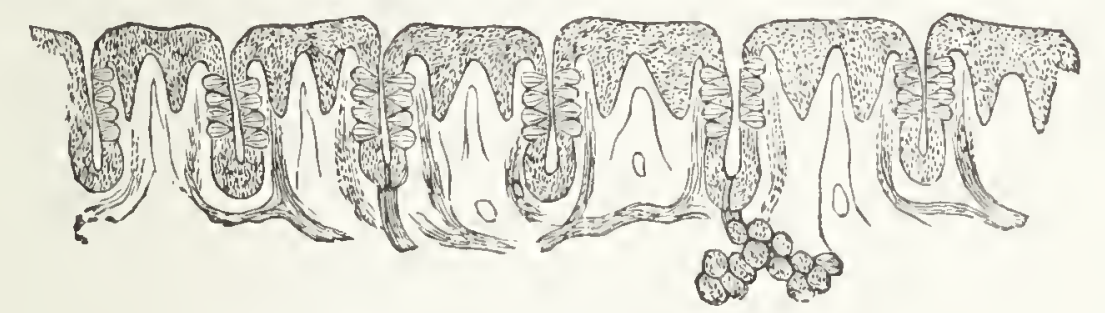

FIG. 174. -Taste-organs in tongue $\times 25$

with large papillæ, called circumvallate papillæe, between which are pits. In the hare and rabbit these pits become more or less parallel leaves and grooves, more easily cut across, and the tongues of these animals are therefore
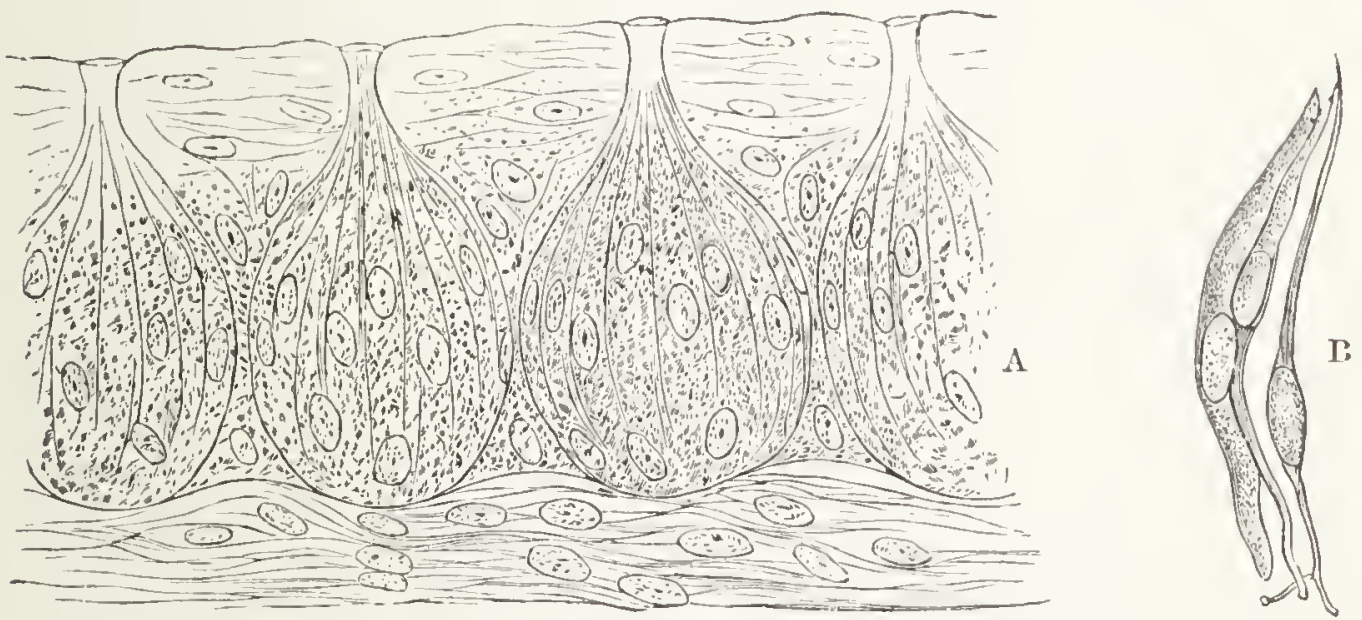

FIG. 175. - Taste-organs $\times 450$. A. Flask-shaped bulbs B. 'Taste-cell with outer cells

usually sectioned to show the taste organs, which resemble minute necked flasks, whose mouths open into the sides of the pits or grooves (fig. 174). The flask-like bulbs are more clearly shown in fig. 175 under a higher power, and it will be seen that the outer part of the bulbs consist of oblong 
cells, each containing a nucleus, and being tapered towards each end. Inside these are the true taste-cells (see righthand cell in B) in which the central portion containing the oval nucleus is drawn out into an exceedingly fine process

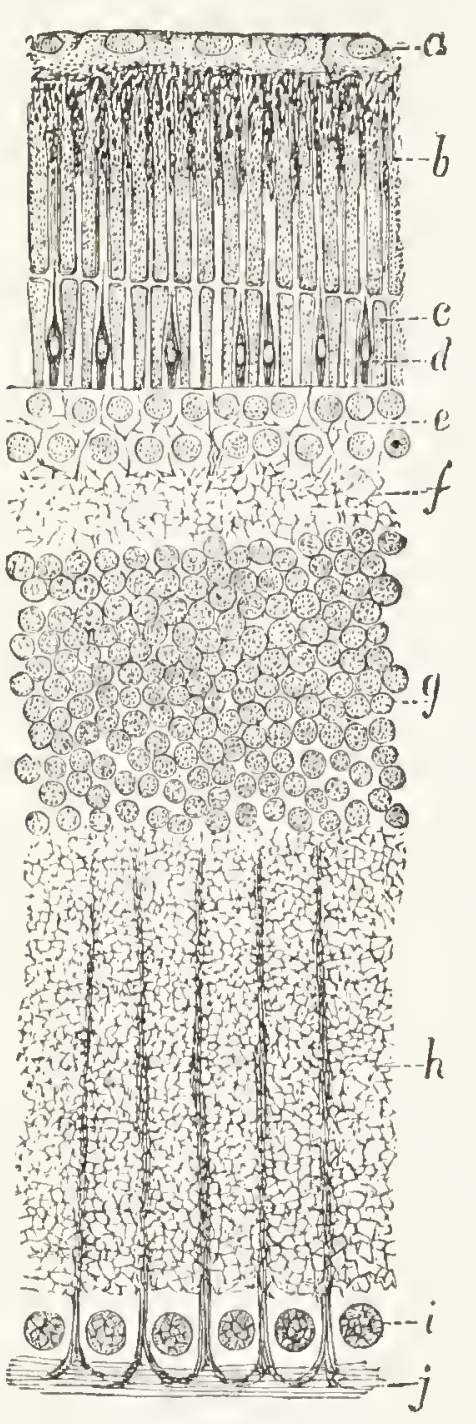

Fig. 176.-Section of Frog's Retina

a. Layer of pigment eells

b, $c, d$. Rouls and eones

$e, g$. Nuelenr cells

$f, h$. Molecular lavers

$i$. Langlion eells $j$. Nerves at each end. The outer end projects a little through the mouth of the flask like a tiny hair, the other is usually branched, and connected with the nerve-fibres below; but the precise mode of connection has even yet hardly been made out.

Our last example is from the nerveendings connected with vision. How an 'image' is thrown upon the retina at the back of the eye-ball, has broadly been shown in fig. 9 ; and it has been indicated in $\$ 22$ that the rays which form this image are inconceivably minute and rapid vibrations in the ether, propagated along the course of the 'ray.' Organs to receive such delicate impressions must themselves be of almost inconceivable delicacy; and fig. 176 is a section of the retina only, from the eye of a frog. Here $j$ are fibres from the optic nerve, and $i$ large ganglion cells connected with them: these ganglion cells and the smaller nuclear cells presently mentioned appear to act in a manner as relays or fresh centres of nervous energy. At $h$ and $f$ are what are called molecular layers, and alternating with them at $e g$ are two layers of nuclear cells, of which it may be remarked that while in the frog the layer at $g$ is much thicker than at $e$, in the human retina the reverse is the case. The outer 
nuclear cells are connected by nerve-fibrils with a layer of extremely fine rocks and cones, thicker at the base $c d$ than the outer ends $b$. These inconceivably fine rods and cones (which are free or unconnected, whilst up to their bases the whole structure is joined by connective tissue) are received universally as the seat of vision. The remarkable thing is that in Vertebrates (owing to that outside-inside embryonic development indicated in $\$ 118$ ) they are at the back of the retina, and the rays have first to pass through all the preceding layers, which are transparent. The analogy is pretty
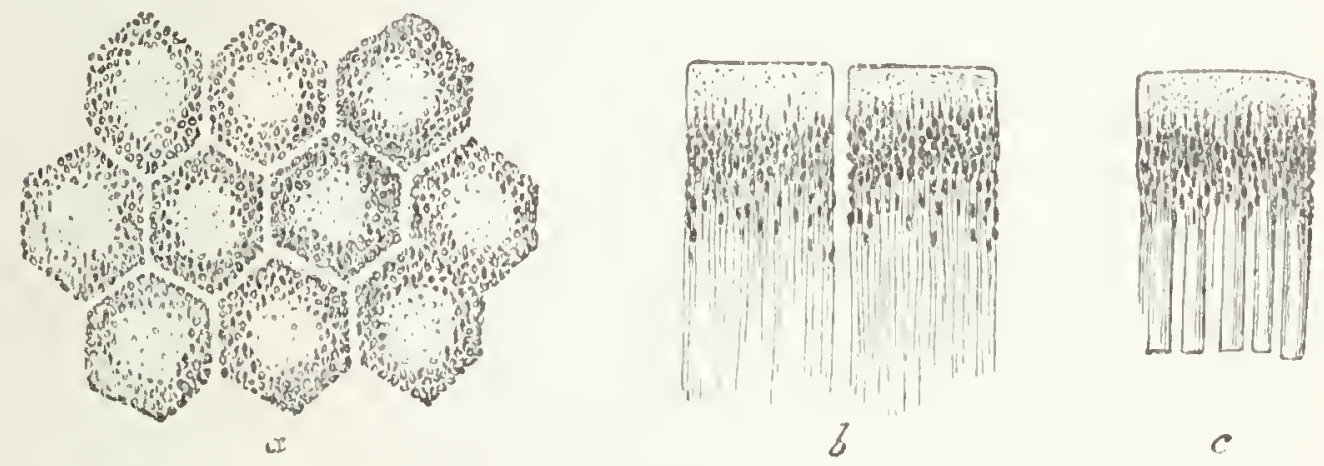

Fra. 177.-Pigment Cells of Retinn

evident between these rods and cones and the cilia of certain epithelial cells ( $\$ 118)$.

At the further end $a$ of the layer of rods and cones is a sort of pavement of pigment cells, hexagonal in shape, as at $a$ in fig. 177. The side next the rods throws out a number of pigmented fibrils $(b)$, which pass down amongst the outer ends of the rods and cones as at $c$. Light causes these fibrils to stretch down farther; darkness to recede. Thoy appear to have an important office. The outer ends of the rods and cones contain a colouring matter termed the visual purple, which bleaches on exposure to the light. But the colour returns when the rods and cones are in contact with the pigment cells, even in the dead retina, and probably also in the living one.

All these details of the intricate and beautiful mechanism are known; but how it acts is a profound mystery still. 
Some think the fine rods and cones 'feel' the vibrations. Others rather believe that the bleaching of the purple, and its renewal by the pigment cells, are of the nature of a photographic process, and that this is vision. But no one knows. No one knows the action of the complicated layers of nuclear cells; no one knows the nature of the defect in the organ which makes some people colour-blind. Defects in the eye as an optical instrument, we can trace out to the last detail, and remedy with our prisms and lenses : the nature of the vital mechanism, no one has yet been able to understand.

The organs of smell and of hearing are equally wonderful in their minuteness of structure; but reference must be made for them to works upon anatomy or histology. Many other organs supply beautiful objects, especially when (as is often done) the blood-vessels are injected so as to show arteries red, and veins blue, the tissues also being stained. Sections of liver and kidney so treated are not only of great beauty in themselves, but will give a realistic sense of the exceeding delicacy of the mechanism of these important secreting organs.

The above will suffice most microscopists for a general collection: some others can only be just mentioned. The various glands and secreting structures of the stomach and intestines are both very interesting and very beautiful, affording some of the clearest and most exquisite examples of the cells of columnar epithelium. A fine illustration of the arrangement of tendons at a joint, is furnished by a longitudinal section of a 'decalcified' joint in the tail of a mouse. A developing tooth, or the entire jaw of a kitten or some other small animal, is a very beautiful and interesting slide. So is a section through the entire eye of a bird, or of a newt; or the entire head of a young newt or frog. This brief list is entirely confined to objects which are almost always to be obtained, most beautifully prepared by the professional mounter. 


\section{CHAPTER XII}

\section{MISCELLANEOUS MICROSCOPIC OBJECTS}

IN this chapter we collect a few notes on various subjects of microscopic study, or classes of objects, all of which are attractive, and some of which secure almost altogether the interest of specialists. We must be content with indications.

123. Diatoms.--One well-known authority has for some time never spent less than $100 l$. a year upon these beautiful objects, which have been termed 'Nature's jewels.' Many microscopists have studied little else for years; and there is no doubt that the attempt to make visible the finer of their markings, ranging in some cases to 100,000 per inch and upwards, has worked marvels in the improvement of microscope lenses. Hence it will be understood that anyone intending to take up this study seriously, must possess first-class objectives, both as regards aperture and definition. But the mere beauty and symmetry of scores of the larger forms can be seen with ordinarily good half and quarter-inch power's.

Diatoms are generally considered unicellar plants, growing in a silicious two-valved case, ${ }^{1}$ and are found in all sorts of

I Though it be heresy to say so, I am extremely doubtful of this. Many year's since I was most unmercifully 'cliaffed' by a small body of microscopists for presumed ignorance in the expressed belief that both Volvox, and at least some of the Diatoms, might probably be ultimately relegated to the animal kingdom. Some of the best recent biologists have already adopted that view as to Volvox: diatoms are not yet so regarded. But the character of the motion in some forms, and of the silicious skeleton in most (it is not clear to me that all so-called Diatoms are on the same plane of existence) arc of great weight from analogy; while I think that in some cases fine pseudopodia can bc observed in the living form. And some of the reasons which were once powcrful in deciding for vegetable character-one for instance was the presence of chlorophyll-are admittedly now of no wcight at all. I have however no title to speak with authority, and merely-for what I think adequate rcason-put my doubts upon record. 
places. In fig. 178 are a group of fossil marine forms, some of which are still found on the surface of the sea. No. 1 is a Surirclla, No. 2 a Pimmularia, No. 3 Gomphonema, No. 4 one of the genus Navicula, No. 5 a cross section or broken piece of Melosira.

Diatoms are also found attached to sea-weeds, as green or orange or reddish scum on the mud at the bottoms of ponds and brackish river-banks near the sea, and in all sorts

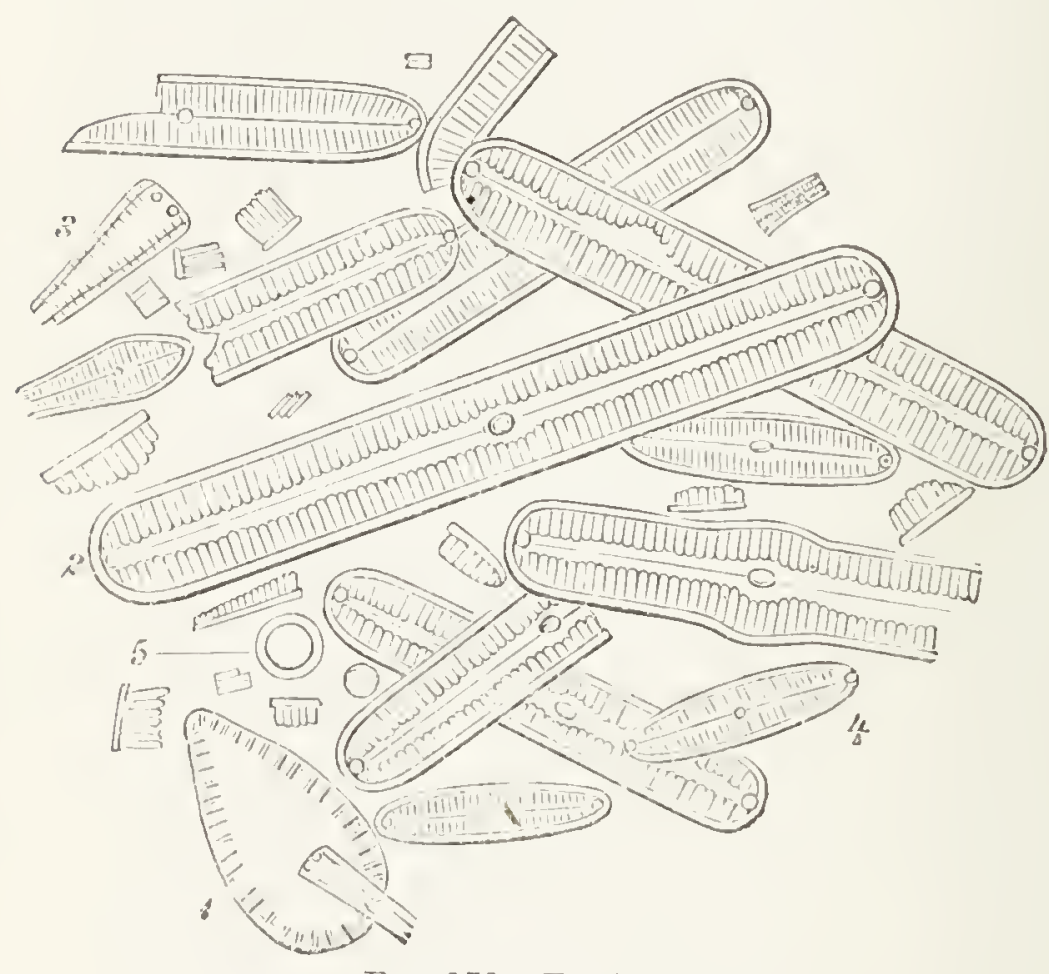

Fic. 178.-Fossil Diatoms of places. The beautiful Arachnoidiscus (fig. 179) is found in places as diverse as upon fronds of seaweed, and stale jam! But the most ample supplies of many forms are fossil, in what are called diatomaceous earths, or in guano. The tooth-powdersold

as 'Zozodont' will furnish for sixpence a large quantity, being a diatomaceous earth, but it varies a great deal; some year or two ago there were many fine forms in it, but the last sample I obtained contained only very common forms, resembling those in fig. 178.

The most beautiful forms are the discoid or equi-angular ones. Fig. 179 reproduces the drawing of the late Mr. Richard Beck of the beatiful Arachnoiliscus, showing both of the two sides of the valve (which are different in all diatoms) and the way in which the two valves are joined into the 'double atom,' in this case resembling a pill-box, the whole 


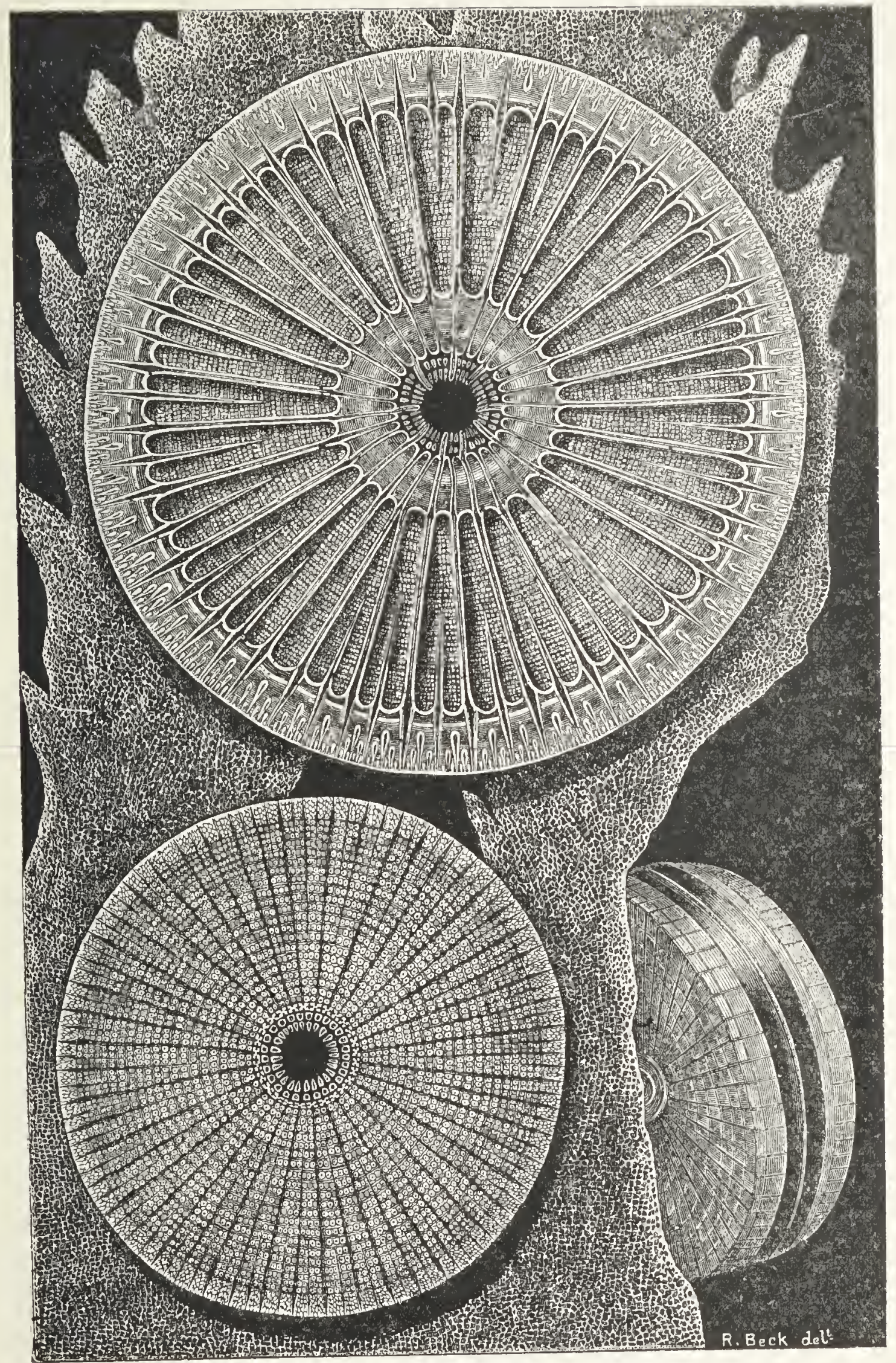

FIG. 179.-Archnoidiscus Japonicus $\times 450$ 
being seen edgeways. There are several varieties of Arachnoidiscus, with no really essential difference: one called A. ornata is perhaps the most beautiful of all. Fig. 180 is another circular Diatom called Heliopelta. There are varieties of this, with a different number of sections in the disk; but all are alike in the fact that the sections are not flat, but undulate; hence the whole valve cannot be in sharp focus at once. This is common to many discoid diatoms;

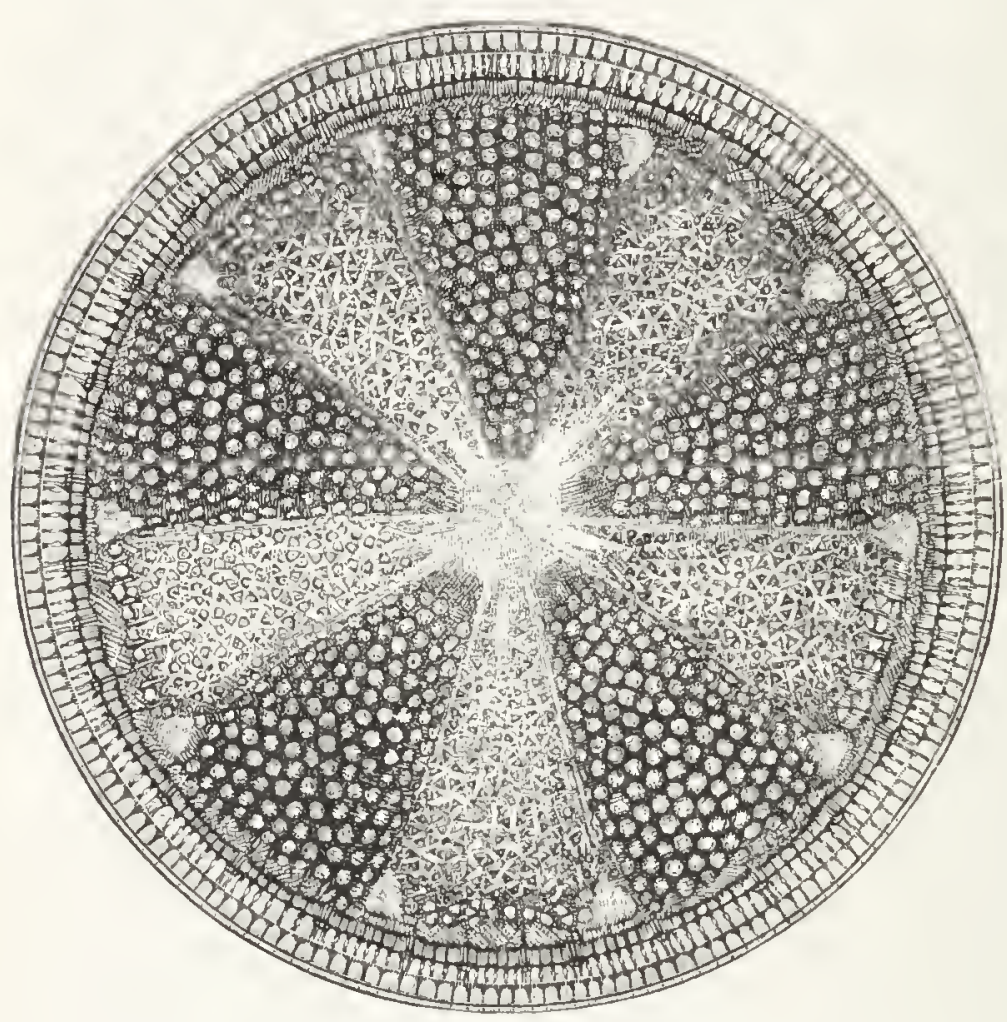

FIG. 180.-Heliopelta $\times 200$ (From photo by Mr. A. A. Carnell.) while others resemble a convex shield. In such the more minute markings can only be focussed in a certain portion at a time.

Fig. 181 is a larger representation of another Navicula (so named from resemblance to the deck or plan of a boat). There are many varieties of $N$. lyra, differing much in coarseness of marking, and one or two are now separated under other names, such as $N$. maculata. Some are much flatter towards the edges than others ; and of every diatom selected, endeavour should be made to secure specimens which lie flat and focus as evenly as possible, either all over, or all round the edge, except when it is desired to show the edge or side view.

Of equi-angled diatoms, Triceratium favus is threecornered, and similar marking but much coarser is found in 
T. septangulatum, with seven corners. A much larger threecornered diatom, still coarser in marking, and with rather bulging sides, is Trinacrium. In all these and many others, inside the larger marking alone apparent under moderate powers, are a number of much smaller markings of various kinds, some of which demand the very highest powers and skilful illumination to make them visible.

Thus, Coscinodiscus astcromphatus is a discoid diatom, which when $\times 100$ appears the size of half a crown (for an average specimen : the size of many diatoms differs greatly) and the portion in focus (for it is not flat) appears studded with dots or spherules (according to focus and excellence of the objective) of which about 40 in line go to an inch; these spots therefore average about 4,000 per linear inch, and are the larger markings. But when $\times 2000$ each 'large' circle is surrounded by a ring of smaller round spots, and within it are a number of much smaller spots. And a most minute difference in focussing, with the same illumination, changes the

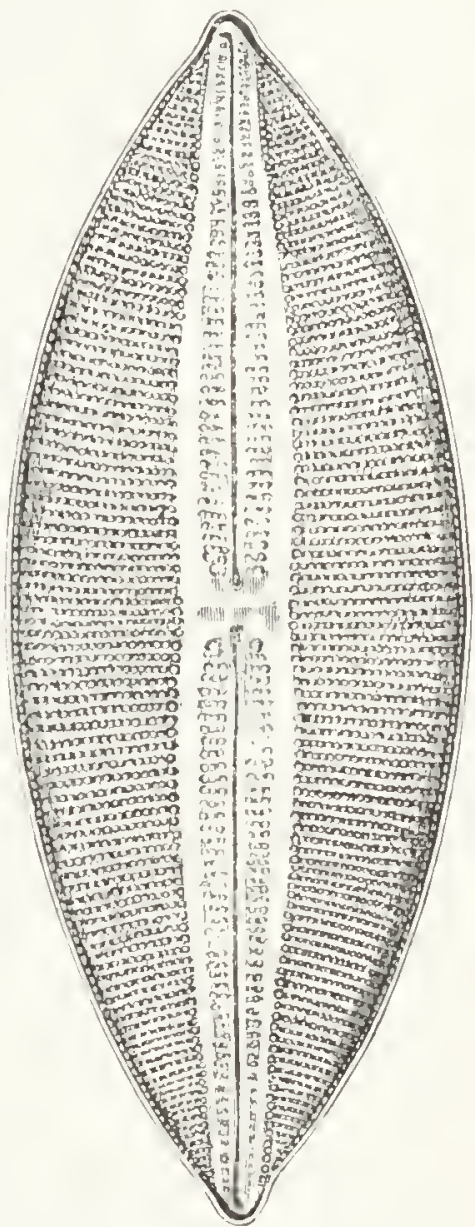

FIG. 181.-Navioula ly'a $\times 600$ (P'hoto by Mr. C. L. Curties.) whole system from white to black, so that one image is like the photographic negative compared with a positive. This class of work, and many of the finer diatoms, require immersion lenses and high-class condensers.

This difference in appearance from a mere shade of focussing, will show the exceeding difficulty of determining diatom structure. It appears as if the very fine dots were on, or apertures through, a very thin layer below or above a thicker one with larger apertures. And the general opinion 
is that the valves of diatoms are composed of either two or three layers. It may differ in various genera, and many good authorities believe that $P$. angulatum consists of apertures in a thicker layer, probably of silex, which is between two inconceivably thin ones, possibly of membrane, or a thin ectoderm. That the small spots are either perforations, or hollows, has been proved by the fact that Mr. C. H. Gill has succeeded in filling them with pigment. The further fact that when by proper arrangements we have got rid of the many spurious 'diffraction-images' caused by a small cone, which in times past have greatly obscured the subject, there remain with a wide aplanatic cone and good objective two opposite appearances and two only-a white spot or a black dotalso seems conclusive that each spot is either an actual aperture, or a section like a very small lens.

Under low powers many diatoms appear very beautiful, but instead of the marking, to be covered with glowing iridescent colour. This is iridescent colour, caused by interferences of the light passing through their regular marking as through a diffraction grating. A higher power, if of sufficient aperture, resolves the flat colour into dots or spherules.

Living Diatoms. - The motion of some of these is very interesting. The best for demonstration are Bacillaria paraloxa, of which a number of the rod-like forms adhere and stide along each other in a curious way : and Pleurosigma quadratus, resembling $P$. angulatum, but much shorter and broader. These move over the slide freely in a drop of water, in a very comspicuous way. Both are to be found pretty easily in the Thames estuary.

Test Diatoms. - For the purposes of this book, these have been sufficiently treated of in $\$ 37$.

124. Foraminifera.-These minute calcareous shells are found of many forms, which exhibit a wonderful variety. Many beds of chalk scores and cven hundreds of feet thick 
are formed of them $(\$ 127)$, but hundreds of species are to be found in their detached form. 'Spread' slides containing many species from the ocean-bed in various localities are always to be obtained, one of the most attractive being the 'Globigerina ooze' brought up in the Challenger expedition from the bottom of the Atlantic. The Mediterranean also furnishes many beautiful forms, and so does the estuary of the Dee.

There are two sources of supply ready to the hand of every microscopist. The first is in new sponges. Everyone knows the sand that soaks out of a newly-purchased sponge. If this be carefully collected and examined, in a large proportion of cases (not of course always) this sand will be found to consist of Froraminifera. These may either be mounted on a black ground in a dry cell, as opaque objects, or spread upon a slip and mounted in balsam.

The other source is the sea-shore. With two or three little bags or bottles, and a spoon, the collector may walk along high-water mark, and low-water mark, and at each level scrape up a little of the cleanest and whitest-looking sand left in the ripples caused by retiring wavelets, in fairly calm weather. Stormy weather mixes things up too much, and the loose sand among the weed at high-water mark also fails for the same cause to yield other than small results. Between high and low water, a little of the sharp whiter sand should also be scraped up where tiny little pools are scooped by the water round the corners of rocks or stones. Of course the Steinheil loup or other pocket lens should be used from time to time, to see if there is anything to be got at each spot attempted. Any chance of dredging off shore in the ooze or mud or sand, is also not to be despised; and the blue clay-mud at the mouths of many estuaries will also yield specimens.

The produce of these proceedings has next to be washed and cleaned. First the salt and mud are washed away in 
several waters. Next the mass is to be passed through two sieves - the top one of perforated zinc or coarse "gauze with meshes or holes about $\frac{1}{8}$ inch, which will take out weed and other large masses; the lower one of wire or silk gauze 120 to 150 meshes per inch. If dirty, boil in solution of potash, then again in water to dissolve the potash out. Finally dry entirely in the oven, and when cool throw into water and stir: the shells being full of air will mostly float on the surface and can be separated, while the solid sand will sink to the bottom. Further sorting out, into sizes or otherwise, can be carried out as desired.

Some of the Foraminifera exhibit well by dark-ground illumination: but as a rule they are best treated as either opaque or transparent objects.

125. Radiolaria.-The most beautiful of these minute forms of life, however, are the silicious or flinty shells of the group of animals named by Ehrenberg Polycystina, but which are since merged into the larger group known as Radiolaria. They are what it is the fashion to call 'singlecelled' animals 'without organs'; nevertheless their leading characteristic is that a central portion is enclosed in a capsule of membrane, within which reproductive growth takes place, while the extra-capsular portion extends pseudopodia, and by these procures food. According as the membranous capsule is pierced by many fine pores, or by one or more larger apertures for communication, the Radiolaria are grouped into two great divisions of Porulosa or Osculosa. Further details of classification camnot be entered into here. ${ }^{1}$

The flinty skeletons of these animals, of which the term Polycystina is still applied to those in which the skeleton is outside the capsule, are as transparent as those of diatoms, and they consequently make splendid objects for dark-ground illumination. Fig. 182 represents a few shells thus shown,

1 The classical work on the subject is Hreckel's magnificent Report on this department of the Challenger expedition. 
reproduced from a fine drawing by Dr. Dallinger. All these forms are very common in a thick bed of silicious sandstone found in Barbadoes, which almost entirely consists of such minute silicious shells. The present living forms appear to live chiefly on the surface of the sea, from which their shells sink after death, forming an 'ooze' at the bottom of the ocean. From this ooze the Challenger

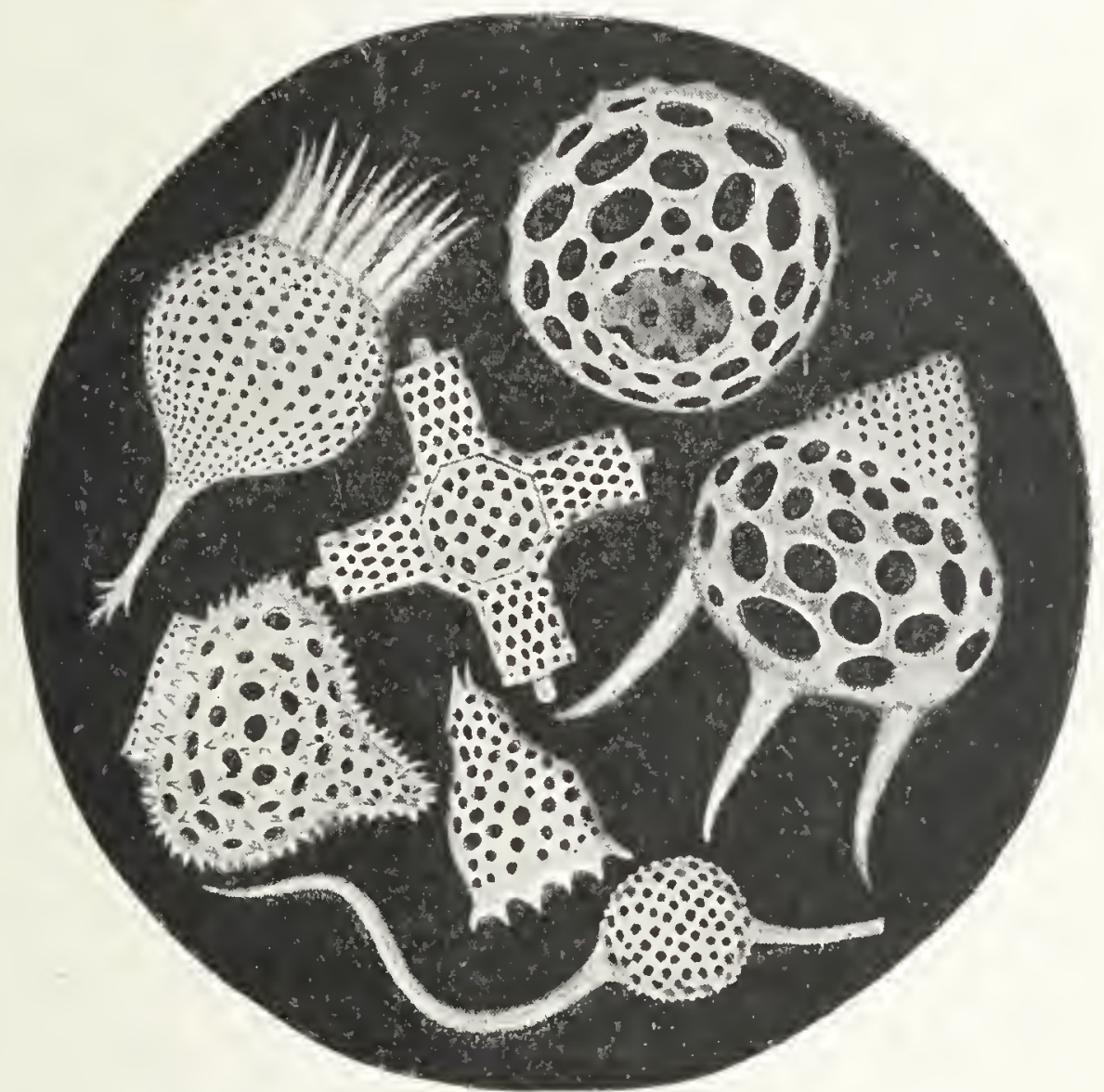

FIG. 182.-Polycystina $\times 100$

expedition has made known thousands of varieties. Such an ooze, if uplifted as dry land by geological change, would doubtless result in a silicious sandstone much resembling that of Barbadoes.

The Frontispiece shows a number of additional forms, all fossil, reproduced from a most beautiful set of drawings made from nature by the late Mr. Mungo Ponton. Many of the specimens figured are of exceeding rarity; and I have 
made selection either for this reason; or for unusual beauty ; (omitting purposely those shown in the drawing by Dr. Dallinger), or lastly, to show by simple ocular demonstration what amazing complexity and minuteness of real structure of some kind must lie hidden in what it pleases biologists like Professor Haeckel to term a 'simple cell,' or 'a speck of primeval slime.' Gradual development is evident enough : for instance, gradual evolution seems clearly written on the forms along the bottom of the page. But what must there be in the 'single cell' which inhabits, and has formed, such a lovely dwelling as the central one of that bottom row; and if his a priori theory of Life leads a man to say there is no 'organisation' there, surely it must be said, So much the worse for the theory! It is certainly not the microscope's fault; it is that of the anti-teleologic, and therefore unphilosophic biologist at the eye-end of the instrument: the microscope itself has done its very best to show him his folly!

126. Echinus Spines.-Thin sections across these are very beautiful and perfectly symmetrical objects, which amongst the various genera and species afford a marvellous variety. In a lecture upon 'Star Fishes' by the late Dr. W. B. Carpenter, which I once had the pleasure of demonstrating for him with the projecting microscope, if my memory serves me aright he had nearly twenty specimens of these sections, besides his other preparations. A few generally find a place in every collection, and exhibit well either by transparent or dark-ground illumination.

127. Mineral Sections.-These are a distinct, large, and interesting class of objects, which furnish a study in themselves. Many of them require polarised light (\$ 40, §58) for their full understanding, and those who pursue this study seriously will need the aid of a properly fitted petrological microscope. But very much interesting detail does not require this. A very. moderate power will suffice to show that most varieties of chalk are almost entirely com- 
posed of the compressed remains of Foraminifcra (fig. 183), and that these and much larger organisms-largely consisting of Encrinites-compose the bulk of many carboniferous limestones (fig. 184). Other organic rocks, fossil Corals and Madrepores, Coal sections, and fossil Woods supply many beautiful objects. Inorganic minerals also frequently display very beautiful phenomena of colour and iridescence,

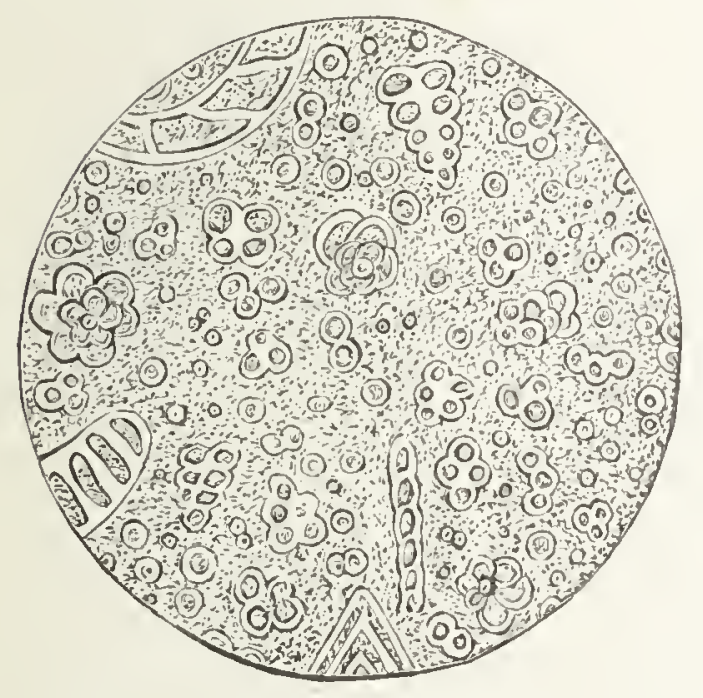

FrG. 183.-Chalk

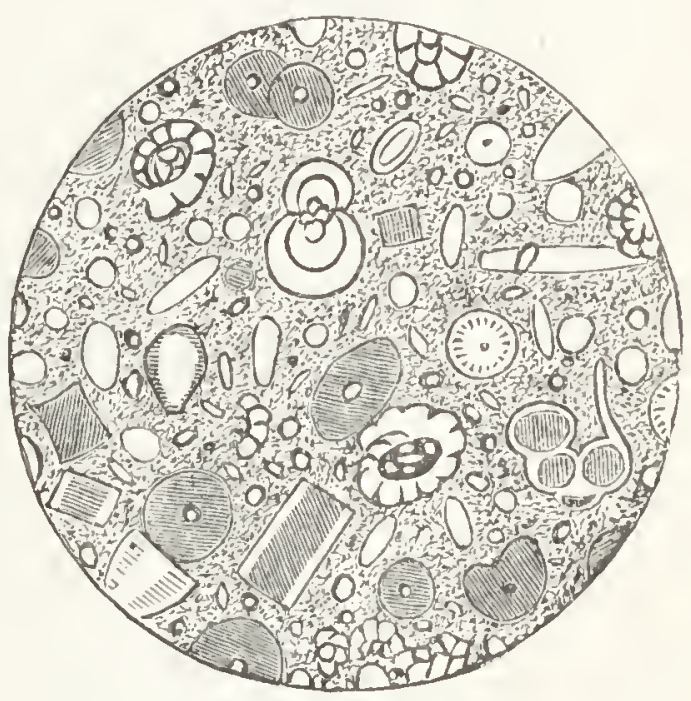

FIG. 184.-Limestone

but as a rule the polariscope is neaded to bring out their beauty.

128. Polarising 0bjects.-Any ordinary section of granite, for instance, is a beautiful object when seen by polarised light (\$185). By ordinary light its main constituents of mica, quartz, and felspar can be fairly made out; but when the polarising prisms are brought into action, their different qualities cause the most beautiful 'play' of colours. Somo of them will give two colours only (always ' complementary' pairs of colours) when the analyser or polariser is adjusted in its two main positions: but other constituents have the property of exhibiting every colour in succession as one of the prisms is rotated. This last phenomenon depends upon the precise direction in which the section cuts a particular constituent part of the mineral; and occasionally some 
minerals-such as amethyst, some agates, chalcedony, and some opals, exhibit bands of these succession colours of enchanting beauty. Any dealer in this class of slides can select an assortment of any extent specially adapted for colour display.

Another class of colour phenomena is shown by sections of many crystals, when polarised light is used in the form

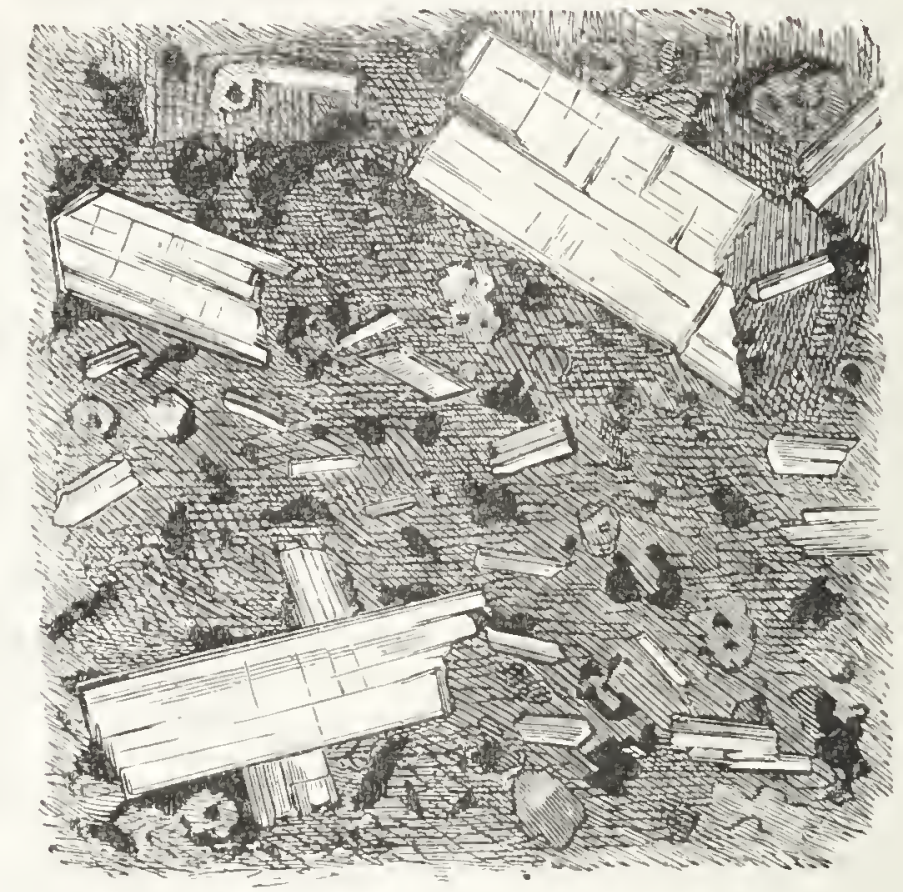

FIG. 185.-Section of Granite by Polarised Light $\times 15$

of a wite cone sent through them by the condenser, the cone crossing in the crystal and being re-collected by a very similar condenser system as an objective. These figures are not images, however, but interference fringes produced by the crystal; and their explanation must be left to treatises upon Petrology or Physical Optics.

Crystal Films of various salts in solution, allowed to crystallise on a glass slip, and mounted when dry, are most beautiful objects, and present most gorgeous colours in polarised light. An immense variety are sold at 1s. each, but the student can purchase the salts and prepare for himself if he prefers. It costs nearly as much, however, by 
the time he has selected a good one out of his various trials. A drop of saturated hot solution spread upon a warmed slip, will enable the process of crystallisation to be watched; but the easiest way to exhibit this pretty phenomenon is to purchase a few slides of the fatty acids, which are prepared for the purpose. These melt at a gentle heat over a lamp, taking care not to crack the glass or melt the ring of varnish; beautiful crystals, in colours, are seen to shoot out as the slide cools. The same slide will serve scores of times, or until some accident happens to it.

If a film of crystals does not happen to show colours alone, the selenite $(\$ 40)$ can be used with it: but the contrasts are better when the colours appear alone. This however depends upon the thickness required of any particular salt. Most crystals will mount in benzol-balsam; but some gradually dissolve in this, and have to be mounted in copal varnish or castor-oil. The beautiful Hippuric acid crystals require the last medium to preserve. The acid is dissolved in absolute alcohol gently warmed, and if a drop is dipped on to a clean warmed slide, it will show the most extraordinary variety of effects, by alternately cooling and warming, breathing on the slide, \&c. Eno's fruit salt, tartaric acid, borax, and carbonate of soda, are common domestic articles that crystallise effectively from aqueous solutions; and will give different effects if hastened over a lamp.

The favourite and beautiful crystallisation of salicine is best prepared by flooding a slip with a saturated solution in warm water containing a little alcohol. This is then held in forceps over a lamp, keeping level, at a moderate heat till the fluid has evaporated. The heat is then increased, when the film melts in its own 'water of crystallisation,' and gradually the beautiful little rosettes start out, the effect being hastened, and varied ad lib., by alternate breathing and cooling and heating in turn. Some salts, such as sulphate of copper, and sulphate of copper and magnesia mixed, melt 
and crystallise in the same way; and heat will modify the crystallisation patterns of tartaric acid and many other crystals.

Organic sections show impressively the power of polarised light in revealing structure. Their variety is countless; and many which exhibit sufficiently well in the ordinary way, come out with added beauty and detail when this additional power of analysis is brought into play. Sections of bone and teeth generally polarise, but without adding much: but sections of hom, hoof, and cartilage are exquisite objects, and develop the details with startling effect. Every variety of fish scale makes an effective polarising object, and is easily cleaned and mounted, either in jelly or balsam. Many hairs polarise strongly; and a bit of horsehair crinoline, or alpaca, cleaned of stiffening or other contamination, and mounted in balsam, makes a very pretty object.

Another class of objects, and one that lends itself to, and opens up another line of, systematic study, may be found in what are commonly called the 'palates' of various molluses, but are more properly known as the 'odontophores.' The

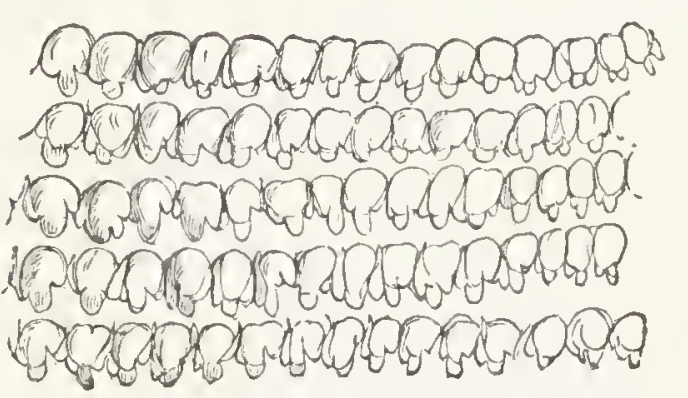

Fic. 186.-Five rows of teeth on Odontophore of Common Garden Snail

organ consists essentially of a cartilaginous strap, bearing a series of rows of hard detached teeth (fig. 1S6). A portion of the strap at the back is generally tubular, and needs to be split up in the preparation. The odontophore is usually curled round at the front of the mouth so as to return back again, and powerful muscles at the two back ends work the front part where it is curled round backwards and forwards, so as to produce a to-and-fro rasping action, which rasps away the substance to which it is applied, even the shells of other molluses, which are often thus cut through.

The teeth and their arrangement differ greatly in different 
molluscs, and a typical set is interesting. After dissecting out the organ it is simmered in hot solution of caustic potash, then well washed from soft integuments and dirty particles. Then it may be treated as other anatomical sections, mounting preferably in jelly for ordinary examination, but in balsam for polarised light.

Of course many vegetable structures also make beautiful objects. Amongst the most characteristic are the starches, every grain of which by polarised light exhibits a black cross, which rotates as the analyser or polariser is rotated.

But the space which can be allotted to enumeration of objects is exhausted. It will be readily understood that almost every division we have mentioned, is a world in itself, if followed up with the aid of any systematic treatise dealing with it: step by step the microscope will go hand in hand with the treatise, and give to it life, and a reality never appreciated before.

\section{CHAPTER XIII}

\section{MICROSCOPICAL EXHIBITIONS}

Anyone known to possess a fairly good microscope, is likely to be asked, sooner or later, to use his resources in the instruction and entertainment of others. Such a request is reasonable, and to comply with it may be a duty; but it very often proves to be anything but a pleasure. I have often observed much pains and trouble bestowed, only to be treated by those who had requested it with such contemptuous indifference as to justify positive refusal on any future similar occasion. A microscopist should guard against treatment of this kind, even for the sake of the 
conversazione or other occasion on which his assistance may be desired. If it is desired, all parties should unite in the endeavour to make it of value. Proper accommodation and facilities should be stipulated for, and some aid towards securing the interest of the company. Granted these essentials, however, either one, or still better several microscopes, are capable of yielding much interest.

129. Precautions.-It is better, as a rule, not to use high powers on such occasions, unless the company is to some extent a skilled one; and for the same reasons exceedingly difficult objects should be avoided. At a regular microscopical soirece, it is different: there, an exhibitor may find it worth while to spend an hour or more in adjusting some special object, which may attract interest for the entire evening amongst the many experts present. Even then, constant vigilance is necessary to prevent serious damage to valuable apparatus by rash handling from those not expert; but how much more in a mere general company. To meet this danger', several opticians actually supply 'dummy milled-heads to go over those working the rack motion, so that the ignorant spectator in turning them may not be able to rack the objective down upon the slide. Such a fact proves how real the danger has been found to be. Moderate powers will be found sufficient, especially with good modern lenses and deeper eyepieces, to show well all that can be shown with profit in a general company.

130. System.-The chief necessity for giving real and living interest to a popular microscopic exhibition, is that it should take place upon some system, and that the idea of the exhibition should be generally known to the company. A regular microscopical conversazione is not here considered. At such it is usual to simply have a card describing each exhibit, which may remain for the whole evening, the company finding their interest in inspecting the exhibits at a hundred or more instruments in succession. We are here 
considering either a single instrument, or a few, up to say half a dozen; and we desire to give corresponding interest to these. Experience proves that this is not done by the common plan of just changing a slide now and then, and in an order the company knows nothing about, except from what they may happen to see upon haphazard inspection.

But now let us systematise the matter. If there be but one instrument, let it be understood that some subject will be illustrated during the evening, and that the slides illustrating it will be changod at set definite times, say from every five to every fifteen minutes. Subjects from the insect world, for instance, are very popular and suitable for such occasions. Going back to our chapter on that subject, either of the two methods there illustrated, or any other, might be adopted. At the stated fixed interval, another slide in the series will be substituted for the last; and the series may be either accompanied by one brief oral description of its main features, given loud enough for the room; or those features can be set out on a card, prepared for each slide beforehand. Everyone will know that at a certain time another item in the series will be ready for inspection, and the whole will have a connected bearing, and really add to intelligent knowledge.

With several instruments, the same plan may be pursued, in either of two ways. The whole team can be brought to bear upon one subject if preferred; and in that case the oral description, given audibly once for all, may be retained. Going again for example to the insect world, the various instruments may display a series of similar organs in various orders, or even genera; or upon the other plan, the prominent organs of one insect might be distributed amongst the whole. These might be then changed for another insect; and so on.

Or each several microscope may follow up during the evening its own individual succession of objects. In this 
case dependence must chiefly be placed upon cards, which should make clear both the nature of the series as a series, and the points of the objects from time to time brought forward to illustrate it.

Other departments, such as pond life, plant life, the anatomy and physiology of the human body, health and disease \&c., lend themselves equally well to such systematic methods of exhibition and brief comment.

But though such is undoubtedly the most satisfactory method of microscopic exhibition, it may often happen that time, or knowledge, or opportunity, or material, may be insufficient to carry it out. In that case the microscopist must trust to the attractiveness of such detached subjects as he can exhibit; and it may be useful to indicate some such, which rarely fail to arouse more or less eager interest. When several instruments are in use, some of these may be worth exhibition during the whole time. Where this is not the case, change should still be made at known intervals, so that spectators may not be in ignorance whether or not they will find something fresh upon another peep.

131. Exhibition Slides.-A little expense is sometimes willingly borne, and a special class of very showy slides are prepared and well known as 'exhibition objects.' Such are symmetrical groups of diatoms, polycystina, spines and anchors, \&c. Some 'spread' slides of the same are of equal beauty. Moderate powers, or dark field, such as will bring out iridescent colours in place of minute marking, will attract more admiration of 'Nature's jewels' than the fine detail of the diatomist as seen under very high power. Eggs of insects are treated in the same way, and some will excite much marvel. Groups representing sprays or bouquets of flowers are also prepared of iridescent butterfly scales, to be shown with the Lieberkühn. Such slides cost from $6 s$. to as much as $3 l$. Some of the groups of eggs, $\& c$., show best in the same way. 
132. Live and Natural Objects.-Mites of various kinds, as from sugar, cheese, \&c., afford a great deal of mere amusement. The heads of living insects, secured as described on page 42 , are generally very attractive, as will be the head and jaws of a spider. If a flea can be procured and starved for a day or two to get transparent, and gently pressed (just to keep it still) in the live box, the beating of the heart or dorsal vessel will be very clearly seen.

An assortment of the smaller flowers, under low powers ranging from 4 inches to 1 inch, and illuminated by the bull's-eye, will often excite the liveliest admiration.

Circulation and cyclosis are always popular ( $\$ 114,116)$. The antenna of the large water-louse (Asellus) shows blood-movement well. A good inch objective will suffice for any of these.

Well-chosen specimens of Pond Life always attract attention. Rotifers, freed from dirty specks by several changes into clean water, and shown on dark ground, are very beautiful: their own instinct keeps them always in the focussed light of the condenser. ${ }^{1}$

133. Plant Structure.-In this department double-stained sections of stems; transverse sections of ovaries, buds, and flowers; various forms of pollen; and cuticles and sëctions of leaves showing stomata, are the most popular subjects.

134. Miscellaneous.-Sections of chalk and foraminiferal limestone, of coal showing organisms, and fossil wood are interesting. With the polariscope, sections of various horny

1 It may be of occasional service to many to state that Rotifers, Polyzoa, Volvox, and any other fresh-water mieroscopic life, can generally be obtained for 1s. per tube from Mr. John Hood, 50 Dallfield Walk, Dundee; or Mr. 'T. Bolton, Balsall Heath Road, Birmingham. Marine Polyzoa, Hydrozoa, \&c., can be obtained alive (and often preserved, or mounted as slides) of Mr. J. Hornell, Biological Laboratory, Jersey (average 1s. 6d. per tube) or of the Director, Marine Biological Association, Citadel,Plymouth (which issues a price list). A very large and comprehcnsive catalogue of slides (the only one of the kind) in all branches of microscopy, published by Messrs. Watson \& Sons, Holborn, will oftcn be found of service. 
tissues, and minerals, and series of crystallisations, are favourites: many crystallised salts are of marvellous beauty. The cornea of an insect's eye arranged to show multiple images of some brilliant object, such as a star in coloured glass, generally excites admiration. More need not be added for the class of exhibition here in view, as experience will readily suggest other subjects.

135. Lantern Exhibitions.-A few sentences must suffice under this head. Insect subjects are peculiarly suitable for projection, and stand out clearly on the screen. In this case something in the way of a lecture will of course be planned. There might be a general review of the Insect World, giving types of organs in various insects; or a single class of organs may be taken, as the wings, or the mouth organs; or such a subject as Household Pests, or Household Insects, or Ponds and Ditches-such are actual subjects which I have myself demonstrated, either in personal lectures or for friends. Minerals demonstrate admirably by polarised light. Botanical subjects exhibit easily and vell, and good double-stained sections are very beautiful. Sections of organs and parts of the human body exhibit well upon the screen up to about 2,000 diameter's with the oxy-hydrogen light, which is sufficient for nearly anything a popular lecturer would desire. In illustrating for a medical friend a popular lecture upon 'The Skin,' I was able to show every point he desired, embracing in all about thirty slides. These brief hints will suffice to indicate what is within the reach of a really good projection instrument and competent demonstrator; and it only need be added, that the Lieberkühn and spot-lens are, for some objects to be shown upon a screen, very valuable and effective adjuncts. 


\section{IN DEX}

Amee, Prof., and the microscope, $28,30,34,35,36$

- condenser, 74

Actinophrys, 153

Actinospherium, 153

Alcyonella, 165

Algæe, 203

Alternation of generations, 204

Amoba, 151

Angle, 26, 28

Antennæe of insects, 192

Aperture, 26, 28

- measure of, 30

- numerical, 30

- and resolution, 31

Aphides, life history of, 199

Apochromatic objectives, 35

Arachnoidiscus, 234

Barrer-animalcule, 149

Bees, 185

Bell-animalcules, 153

Benzol, mounting in, 127

Binocular microscopes, 63

Blood, 218

- circulation of, 220

Bone, 225

Brachionus, 159

Breeze-fly, mouth of, 191

Bull's-eye condensers, 86

Butterfly eggs, 194

Camera lucida, 109

Capillary system, the, 221

Care of apparatus, 83

Cells, 124

— ciliated, 221

- life of, 150

- vegetable, 200
Cement, 124

'Challenger' expedition, objects from, 239, 241

Chirodota, 169

Chitine, 173

Chromatic aberration, 23

Ciliated cells, 221

Cleaning instruments, mode of, 83

Closteria, 202

Cockroach, the, 182

- mouth of, 188

Coleps, 149

Collar correction, 107

Collecting stick, 139

Compound microscope, 21

Condensers, 74

- use of, 92

Cones, illuminating, 95

- ground glass, 97

Conjugation, 202

Conochilus, 162

Corallines, 170, 171

Cordylophora, 165

Corethra plumicornis, 167

Corpuscles of blood, 218

Coryne fruticosa, 170

Coscinodiscus, 237

Cover-glass, effect of, 25

Cristatella, 166

Critical angle, the, 15

- image, a, 105

Crystallisations, 244

Cyclops, 147

Cyclosis, 202, 216

Cynips, life history of, 199

Dantinger, Dr., on protoplasm, 152

Daphnix, 142 
Dark-ground illumination, 100

Dendrosoma, $156^{\circ}$

Desmids, 201

Diatoms, living, 238

- mounting of, 233

- testing by, 71, 106

Diffraction spectra, 33

Dissecting, mode of, 121

- microscopes, 39

Drag hook, the, 140

Drawing, 109

Drone-fly, mouth of, 190

Dropping tube, 120

Dry objects, mounting, 125

Ectimoneris, 169

Echinus spines, 242

Eggs of insects, 194

Entomostraca, 142

Euglena, 158

Euplectella, 169

Eye of frog, 230

- parts of, 230

Eye-pieces, 72

- shades, 82

Eyes, care of the, 81

Ferns, 216

Fertilisation of flowers, 210

Floscules, 160

Flowers and the simple microscope, 41

Foraminifera, 233

Forceps, 117, 121

Frog, eye of, 230

Fungi, 207

Gatl flies, history of, 199

Glass, mode of cleaning, 124

Glycerine jelly mounts, 130

Gnat, larva of, 167

Goat-moth caterpillar, legs of, 181

Gonophores, 170

Grasshopper, great green, 185

Ground-glass illumination, 89, 97

Gum, use of, 126

Gyrinus, 183

HaIr, 226

Hairy midge, the, 186

Haversian canals, 225
Hearing, organs of, 232

Heliopelta, 236

High powers, precautions with, 90

Histological microscopes, 54

House fty, the, 173 ; legs of, 174 ; twings of, 176 ; spiracles of, 176 ; head of, 177; antennæ, 178; eye of, 179

Hydra, 163

Hydrozoa, 163

ILLUMINATING apparatus, 74

Illumination, dark-ground, 100

- oblique, 98

- opaque, 103

Images, 11 ; size of, 13 ; and lenses, 18 ; critical, 105

Immersion, 28

— homogeneous, 29

Infusoria, 148; ciliated, 149 ; flagellated, 150 ; tentacled, 156

Insects shown by simple microscope, 42 ; study of, 172 ; eggs of, 174; legs and feet of, 181 ; wings of, 186; mouths of, 188 ; antennæ, 192 ; ovipositors, 192

Inversion of images, 13

IiNife, the hooked, 139

Irnives, 121

Licinulatia, 162

I amps, 84

Lantern exhibitions, 252

- microscopes, 80

Leaves of plants, 215

Legs of insects, 181

Lens, action of a, 16

Lenses, and images, 18; aberration of, 22 ; single and compound, 37

Life histories, 198

Light, mode of using, 84

Live box, 118

- objects, 118

Joog-wood staining, 133

Lophopus, 166

Lucernaria, 170

Magnification, 11

Manipulation, 81, 87

Maytly, larva of, 167 
Measurement, 110

Megalotrocha, 162

Melicerta, 161

Micrometers, 110, 111

Microscope, the, simple, 20 ; compound, 21 ; power of, 22 ; aberration of lenses, 22 ; chromatic aberration, 23; objectives, 24 ; effect of cover-glass, 25 ; angle and aperture, $26,28,31$; immersion objectives, 27 ; homogeneous immersion, 29; measure of aperture, 30 ; numerical aperture, 30 ; resolution, 31 ; diffraction spectra, 33 ; apochromatic objectives, 35 ; compensating eye-pieces, 36 ; dissecting, 39 ; use of simple, 40 ; flowers under, 41 ; insects under, 42 ; modern compound, 44 ; choice of, 45 ; parts of, 47 ; foot, 47 ; stage, 48 ; tube, 49 ; adjustments, 50; mirror, 51 ; sub-stage, 51 ; stands, 52 ; 'Star,' 54 ; histological, 54; 'Edinburgh,' 59; 'Economic,' 62 ; 'Paragon,' 62 ; binocular, 63 ; objectives, 65 ; apochromatic objectives, 67 ; qualities of objectives, 68 ; testing objectives, 70 ; eye-pieces, 74 ; illuminating apparatus, 74 ; polarising apparatus, 77 ; lantern, 80 ; manipulation of, 81,87 ; care of the apparatus, 83; cleaning, 83 ; light for, 84 ; bull's-eye condensers, 86 ; precautions with high power, 90 ; searching slides, 90 ; nose-piece, 91 ; use of condensers, 92 ; illuminating cones, 95; ground g]ass cones, 97 ; obliqueillumination, 98 ; dark-ground illumination, 100; opaque illumination, 103; polarised light, 104 ; various methods, 105 ; a critical image, 105 ; collar correction, 107; with camera, 114; use of, in exhibitions, 247

Microscopical exhibitions, precautions for, 248; system for, 248 ; slides for, 251 ; live and natural objects for, 251 ; plant structure, 251 ; lantern, 252

Microtome, the, 132

Mildew, wheat, 205

Mineral sections, 212

Mosses, 207

Mounting slides, 123; dry slides, 125 ; in benzol, 127

Mouths of insects, 188

Muscle, 224

Naturai objects, 117

Navicula, 236

Needles, 121

Nerve endings, 228

Net, the, 138

Nit coralline, 171

Nose-pieces, 91

OAK-APPLE, cause of, 199

Objectives, 24, 65; immersion, 27; apochromatic, 35, 67; qualities of, 68 ; testing, 70

Odontophores, 246

Oil, protection against, 131

Ophrydium, 155

Organic sections, 216

Ovipositors of insects, 192

Palates, 246

Parabolic reflector, 10:3

Paraboloid, 101

Paramecium, 149

Parasites, eggs of, 194

Peristomes of mosses, 208

Photography, 111

Plants, leaves of, 215

- stems of, 213

— vessels of, 213

Plumatella, 165

Pocket lens, uses of, 43

Podura test, 70

Polarised light, 104

- objects, 242

Polycystina, 240

Polynema natans, life of, 198

Polyzon, 141, 165

Pond life, collection of, 138

Power of a microscope, 22

Prism action of a, 16 
Protoplasm, 150

Psychoda, 186

RADIOLARIA, 240

Reflection, total, 15

Reflector, parabolic, 103

Refraction, 13

- law of, 14

Resolution, 31

Rotifers, 141, 158

- clustering, 161

-- tube-dwelling, 160

Rust in wheat, 205

Saticine crystals, 245

Saw-fly, 193

Sea-oak coralline, 170

Searching slides, 90

Section-cutting, 131

Sections, staining, 133

- charred, 135

- handling, 135

Sertularia, 170

Sex, development of, 208

Sirex gigas, ovipositors of, 193

Skin, 226

Slides, exhibition, 250

— mounting, 123

- storage and selection of, 135

Smell, organs of, 232

Sphæroplea, development of, 208

Spiders, 195

— webs of, 197

Spirogyra, 203

Sponges, 168

Spot lens, 100

Stage forceps, 117

Staining sections, 133

- - in red and green, 134

Stands, microscope, 52

Starches, 247

Steinheil lenses, 39
Stems of plants, 213

Stentors, 154

Stephanoceros, 161

Stick, the collecting, 139

Stops for condenser, 100, 102

Storage of slides, 135

Sun-animalcules, 153

Swan-animalcule, 149

Synapta, $\mathbf{1 6 9}$

Tabande, 191

Tanypus, larva of, 182

Taste-cells, 230

Teleutospores, 207

Tentaculifera, 156

Tests for objectives, 70

Thread cells, 164

Touch-bodies, 229

Triceratium, 236

Troughs, 119

Trumpet-animalcules, 154

Tubifex, 167

'Tuin-table, Shadbolt's, 124

VALLISNERIA, 217

Vaucheria, development of, 209

Venus' flower basket, 169

Vessels of plants, 213

Vision, organs of, 230

Volvox, 156

Vorticella, 153

WaTER bear, 167

- beetles, 182

- fleas, 142

- scorpion, 184

UVebs of spiders, 197

Wheat rust, 205

Whirling beetles, 183

Wings of insects, 186

Work, mode of, 87

PRINTED BY

SPO'lISWOODE AND CO, NEW-STRFET SQUARE 





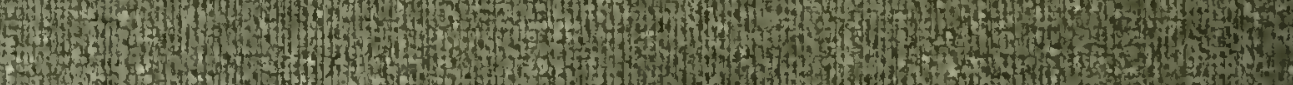

1.7.

12.3.

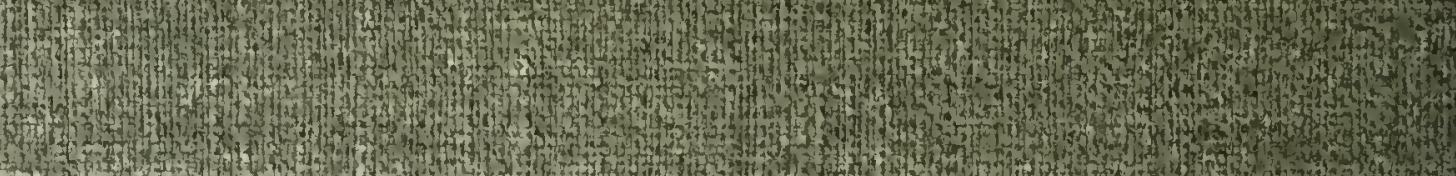

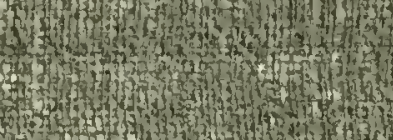

